SOUTHWEST RESEARCH INSTITUTE

Post Office Drawer 28510, 6220 Culebra Road

San Antonio, Texas 78284

\title{
FEASIBILITY INVESTIGATION AND DESIGN STUDY OF OPTICAL WELL LOGGING METHODS FOR HIGH TEMPERATURE GEOTHERMAL WELLS
}

by

R. K. Swanson

R. E. Anderson

J. Iran Ash

R. E. Beissner

V. Devon Smith

prepared for

Division of Geothermal Energy

Department of Energy

Washington, D. C. 20545

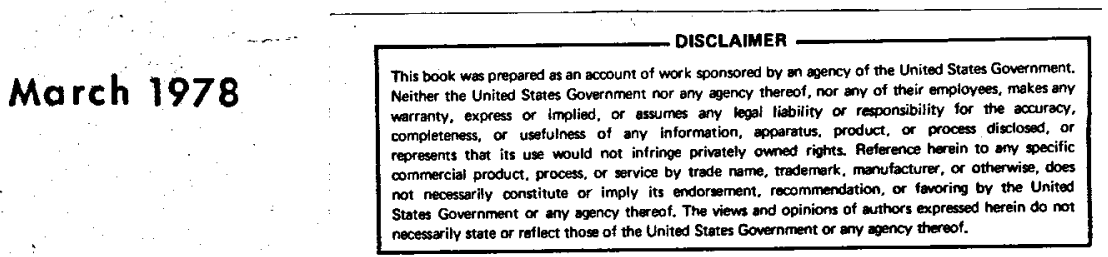

Approved:

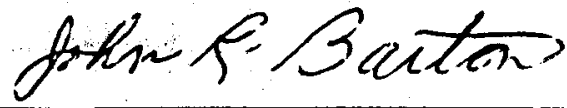

John R. Barton, Vice President

Instrumentation Research Division 


\section{DISCLAIMER}

This report was prepared as an account of work sponsored by an agency of the United States Government. Neither the United States Government nor any agency Thereof, nor any of their employees, makes any warranty, express or implied, or assumes any legal liability or responsibility for the accuracy, completeness, or usefulness of any information, apparatus, product, or process disclosed, or represents that its use would not infringe privately owned rights. Reference herein to any specific commercial product, process, or service by trade name, trademark, manufacturer, or otherwise does not necessarily constitute or imply its endorsement, recommendation, or favoring by the United States Government or any agency thereof. The views and opinions of authors expressed herein do not necessarily state or reflect those of the United States Government or any agency thereof. 


\section{DISCLAIMER}

Portions of this document may be illegible in electronic image products. Images are produced from the best available original document. 


\section{SUMMARY}

This document reports the activities conducted by Southwest Research Institute under sponsorship of the Department of Energy, Contract E(40-1)-5268. The objective of this project was exploration of a novel approach to high temperature well logging, based on a system of optical transducers and an optical transmission line both theoretically capable of operation to at least $600^{\circ} \mathrm{C}$. The scope of the work involved the accomplishment of ten specific tasks. These had as their objective the determination of feasibility, and identification of major problem areas, in the implementation of continuous temperature logging of geothermal wells using optical techniques. These tasks, together with brief summaries of the effort included in each and references to appropriate sections of the report covering the work, are listed below.

Task 1. Literature Review and Data Compilation -A full survey of the open literature was conducted through the facilities of the Nondestructive Testing Information Analysis Center (NTIAC) operated by SwRI for the Department of Defenşe. A total of more than 2500 titles were scanned. The pertinent results of the survey are contained in 31 specific references in the main report bibliography, and 12 references at the end of Appendix $C$.

Task 2. Measurement of Fiber Properties-A method of measuring optical transmission effects in short lengths of optical fibers under conditions of applied stress and elevated temperature was developed, and effects of tensile loading and temperatures as high as $500^{\circ} \mathrm{C}$ were measured on a specific low-loss optical fiber. The work is detailed in Section IV.C.

Task 3. Production Fiber Procurement-Considerations affecting the selection of fibers to be used in constructing a well-logging cable were defined and sample fibers obtained. The work is detailed in Section IV.A.

Task 4. Investigation of Methods of Fiber Termination - A cable head design was completed and individual components procured and tested. Pressure seals for sealing individual buffered fibers at temperatures of $270^{\circ} \mathrm{C}$ and pressures as high as 7500 psi were demonstrated. The work is detailed in Section V.

Task 5. Cable Design and Fabrication-An optical well-logging cable containing three individual optical fibers and three electrical conductors was designed and construction attempted. Difficulties in applying a sufficiently heavy coat of high-temperature buffer material prevented the completion of the cable prototype. Problems were adequately defined and an assessment made of the approach. This work is detailed in Section IV.D.

Task 6. Sensor Performance Predictions-A temperature logging sensor based on measurement of the optical absorption edge in a compound semiconductor was conceived, and its operation theoretically analyzed. Analysis showed that such a sensor would be capable of performing to temperatures above $500^{\circ} \mathrm{C}$, at optical wavelengths within the principal transmission bands of quartz fibers. The analysis is described in detail in Section VI.A.1, 2 and 3.

Task 7. Experimental Investigation-Experiments were conducted with temperature sensing elements constructed of gallium arsenide ( $\mathrm{GaAs}$ ). Results established the feasibility of the concept, and resulted in a practical sensor design. The experiments are detailed in Section VI.A. 4, 5, and 6.

Task 8. Sensor Design - Two complete temperature logging elements were designed and constructed, utilizing GaAs. The design effort is described in Section VI.A. 7. 
Task 9. Evaluation of Complete System - The complete temperature logging system, including sensor, surface transmission source, and surface detection equipment was operated successfully in the laboratory, the sensor at temperatures as high as $275^{\circ} \mathrm{C}$. Because the optical cable was not completed, overall system evaluation could only be simulated. This evaluation is discussed fully in Section VI.A. 8 .

Task 10. Analysis of Advanced Sensor Concepts-Additional effort was devoted to the development of an optical neutron logging concept, and a preliminary demonstration was accomplished. In addition, several promising approaches to pressure measurement by optical means were described. Neutron sensor development is detailed fully in Section VI.B. while pressure transducer concepts are discussed in Section VI.D.

Additionally, during the course of the project, it was suggested by the Contract Monitor that some effort be devoted to a preliminary evaluation of combination electro-optic systems.

Task 11. Investigation of Electro-Optical Systems-Emitter and modulator concepts applicable to down-hole instruments for interfacing electronic packages to the optical system, were given preliminary evaluation. Literature references to at least two modulator concepts were located. A solid-state optical emitter was demonstrated to temperatures above $200^{\circ} \mathrm{C}$, with projected operation to temperatures as high as $300^{\circ} \mathrm{C}$. Work on electro-optic systems is discussed fully in Section VI.C.

A detailed summary of project results, conclusions and recommendations appears in Section VII.

The planned work was successfully completed, with the exception of the construction of a prototype cable, and its subsequent evaluation. This proved to be a much more extensive undertaking than originally anticipated. The need to isolate individual fibers with a heavy buffer coating to minimize microbend losses, creates a severe problem when high-temperature plastics are required. Satisfactory means of applying PFA, the selected high temperature material, to the fibers in sufficient thickness could not be developed within the limited scope of this project. The problems are now well defined, and a cable development program of adequate scope is one of the pressing recommendations to be inferred from the results of this investigation.

The feasibility of continuous temperature logging by passive optical means has been demonstrated and can be implemented, provided the cable problems can be solved. Other sensor concepts, including optical neutron logging, offer excellent promise. The integration of temperature hardened electronics with an optical logging system can be accomplished, using opto-electric modulators or solid-state infrared emitters, such as Si doped GaAs LED's.

Expanded effort in the development of an optical cable, with subsequent implementation of an experimental optical temperature logging system is recommended. Further development of advanced optical sensors, including the neutron logging system and pressure sensors, should also be subjects for additional effort.

The authors particularly wish to acknowledge the encouragement and cooperation of Mr. Larry Ball and Mr. Clifton Carwile of the Division of Geothermal Energy, DOE, and Mr. Michael Lamers, the contract monitor. 


\section{TABLE OF CONTENTS}

Page

I. INTRODUCTION $\ldots \ldots \ldots \ldots \ldots \ldots \ldots \ldots \ldots \ldots \ldots \ldots \ldots \ldots \ldots \ldots \ldots$

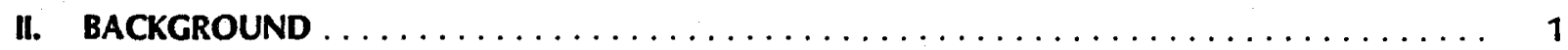

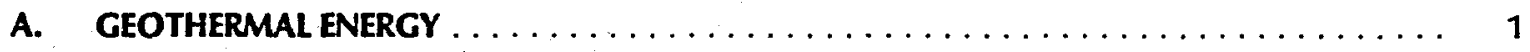

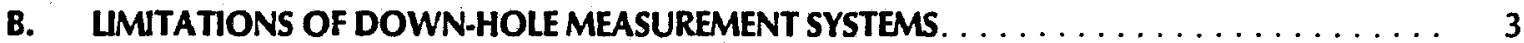

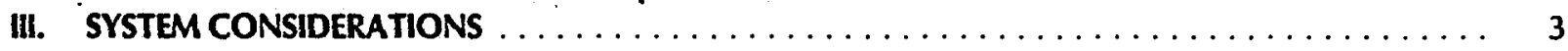

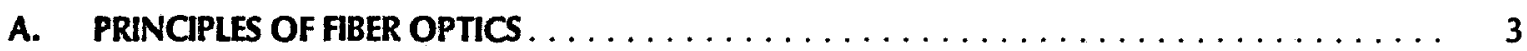

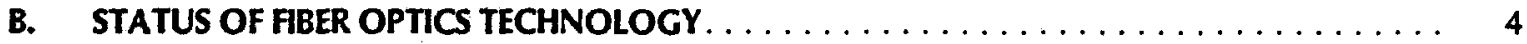

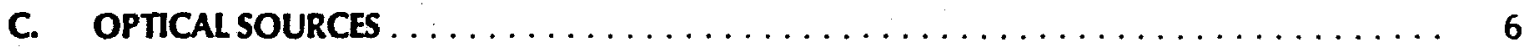

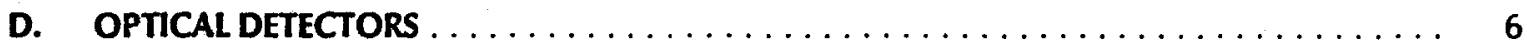

IV. Cable design and fabrication. $\ldots \ldots \ldots \ldots \ldots \ldots \ldots \ldots \ldots \ldots \ldots \ldots \ldots \ldots$

A. SUBCONTRACTOR SELECTION $\ldots \ldots \ldots \ldots \ldots \ldots \ldots \ldots \ldots \ldots \ldots \ldots \ldots \ldots$

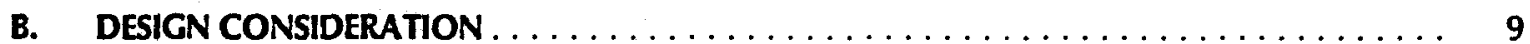

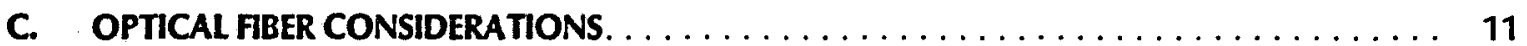

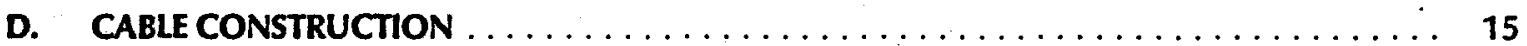

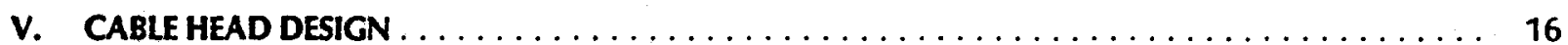

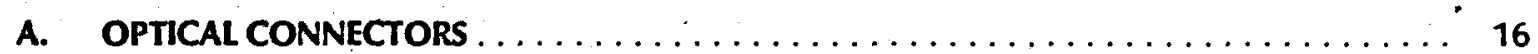

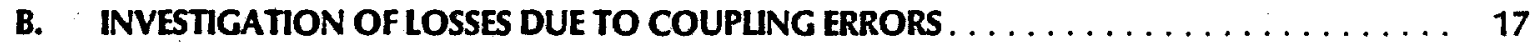

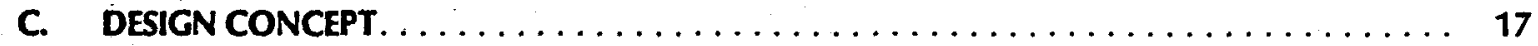

D. PRESSURE CONNECTOR DESIGN $\ldots \ldots \ldots \ldots \ldots \ldots \ldots \ldots \ldots \ldots \ldots \ldots \ldots \ldots$

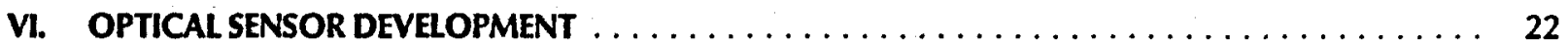

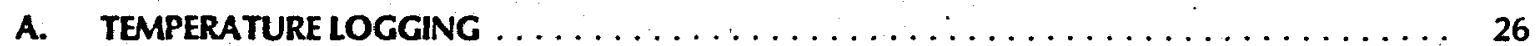

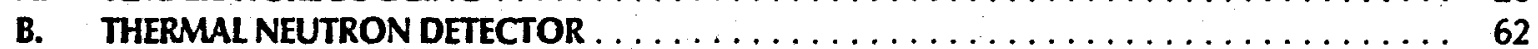

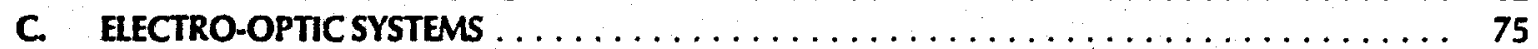

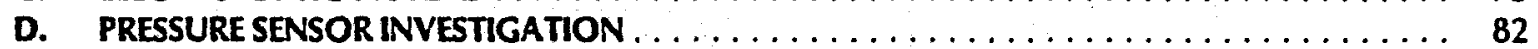

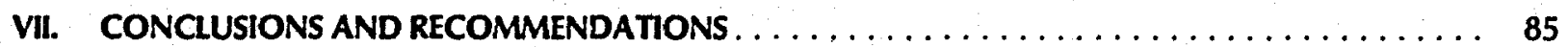

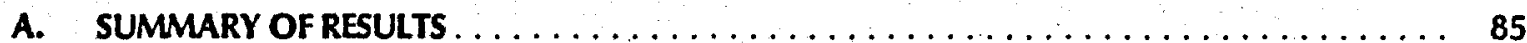

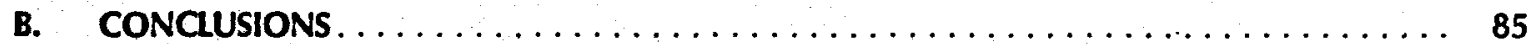

C. RECOMMENDATIONS $\ldots \ldots \ldots \ldots \ldots \ldots \ldots \ldots \ldots \ldots \ldots \ldots \ldots \ldots$

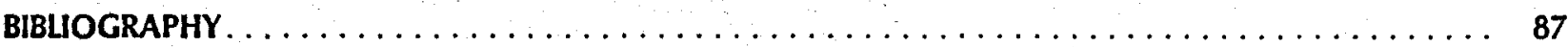

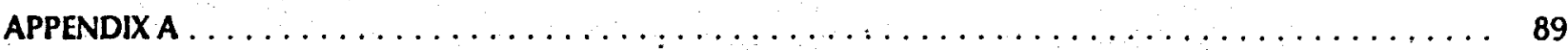

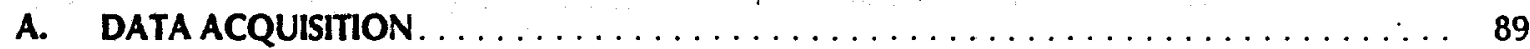

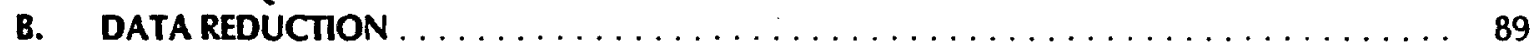

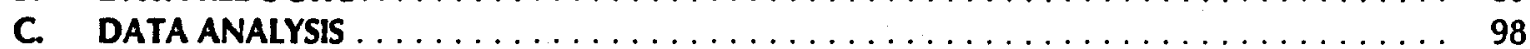




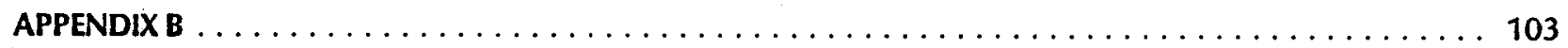

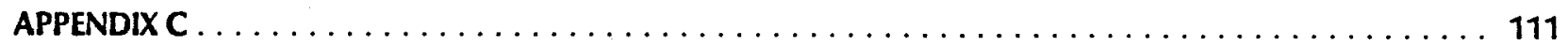

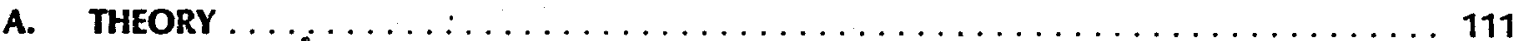

B. EXPERIMENTAL INVESTIGATION $\ldots \ldots \ldots \ldots \ldots \ldots \ldots \ldots \ldots \ldots \ldots \ldots \ldots \ldots \ldots \ldots \ldots$

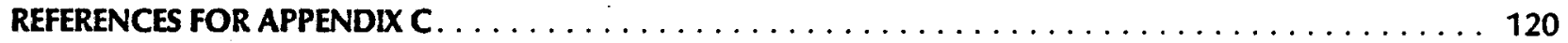




\section{LIST OF ILLUSTRATIONS}

Figure $\quad$ Page

1. Schematic of the Basic Optical Logging Concept. $\ldots \ldots \ldots \ldots \ldots \ldots \ldots \ldots \ldots \ldots$

2. Clad Quartz Optical Fiber Illuminated by Microscope Lamp $\ldots \ldots \ldots \ldots \ldots \ldots \ldots \ldots$

3. Samples of Low-Temperature Armored Fiber-Optic Cables $\ldots \ldots \ldots \ldots \ldots \ldots \ldots \ldots$

4. Tentative High-Temperature Cable Design, Combining Three Optical and Three Electrical Paths. . . . . . . . . . . . . . . 10

5. Schematic of Apparatus for Determination of Optical Transmission Properties of

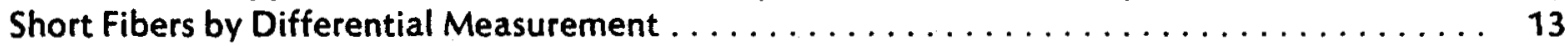

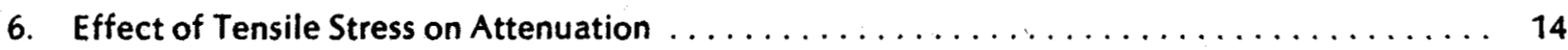

7. Simplified Assembly Drawing of Optical Cable-Head $\ldots \ldots \ldots \ldots \ldots \ldots \ldots \ldots \ldots$

8. Schematic Drawing of Optical Connector Held Inside the Pressure Housing . . . . . . . 19

9. Photograph of a Slotted Ferrule, One of the Photo-Etched Plates, and a Stripped 0.068 -inch Buffered Fiber . . . . . . . . . . . . . . . . . . . . .

10. Miniature Pressure Chamber and Pressure-Barrier Test Fixture $\ldots \ldots \ldots \ldots \ldots \ldots \ldots \ldots$

11. Photomicrograph (45X) of TFE-on-PFA Pressure Seal After Pressure Test $\ldots \ldots \ldots \ldots \ldots \ldots$

12. Pressure-Temp Plot for TFE O-Ring Seal on PFA Buffered Optical Fiber $\ldots \ldots \ldots \ldots \ldots \ldots$

13. Decrease in Optical Absorption Caused by Annealing of $\mathrm{M}$ Centers in $\mathrm{KCL} . \ldots \ldots \ldots \ldots$

14. Experimentally Measured Optical Transmission of GaAs vs. Wavelength and Temperature. . . 30

15. GaAs Absorption Edge at $300^{\circ} \mathrm{C}$, Expanded Scale $\ldots \ldots \ldots \ldots \ldots \ldots \ldots \ldots \ldots \ldots \ldots$

16. Plot of the Theoretical Absorption Edge in Callium Arsenide at Room Temperature . . . . . . 34

17. Plot of the Slope of Theoretical Absorption Spectrum in Gallium Arsenide at

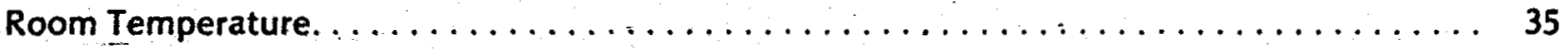

18. Plot of Light Transmitted Versus Thickness at the Maximum Slope-Point of the

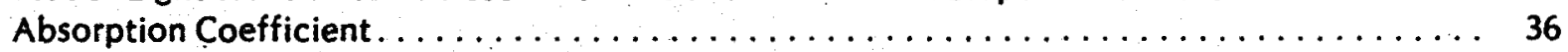

19. Absorption Spectra on N-Type GaAs $\left(\mathrm{N}=7 \times 10^{17} \mathrm{~cm}^{-3}\right)$ Obtained at Various Temperatures $\mathrm{T}\left({ }^{\circ} \mathrm{K}\right):$ 1) $300 ;$ 2) 473 ; 3) 673 ; 4) 873; 5) $1073 \ldots \ldots \ldots \ldots \ldots \ldots \ldots \ldots$

20. Schematic Representation of Apparatus for Measurement of Temperature Characteristics of $\mathrm{GaAs}$ as an Optical Element $\ldots \ldots \ldots \ldots \ldots \ldots \ldots \ldots \ldots \ldots \ldots \ldots \ldots \ldots$

21. Experimental Apparatus Shown Schematically in Figure $20 \ldots \ldots \ldots \ldots \ldots \ldots$ 
22. Temperature Variation Data Corrected for Source Spectrum for the Original Callium

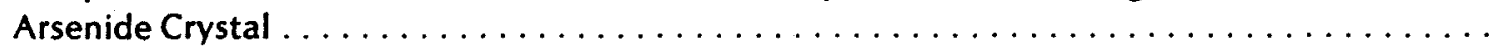

23. Temperature Variation of the Absorption Edge of the Original Gallium Arsenide Crystal. .....

24. Temperature Variation of the Absorption Edge Slope of the Original Gallium Arsenide Crystal . 43

25. Variation of Inverse Critical Wavelength with Temperature for GaAs . . . . . . . . . 44

26. Absorption Edge for (1) Undoped (2) 10119/cc Zn Doped and (3) 10118/cc Zn Doped GaAs . . . 45

27. Slope of Absorption Edge for (1) Undoped (2) 10119 and (3) 10118/cc Zn Doped GaAs. . . . . . 46

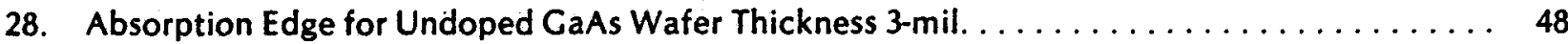

29. Photomultiplier-Threshold Method of Determining Cut-Off Wavelength for GaAs . . . . . . . 49

30. Experimental Arrangement for Making Photomultiplier Threshold Measurements with GaAs Temperature Sensor . . . . . . . . . . . . . . . . . . . . . . . . . 51

31. Experimentally Derived Temperature Calibration for GaAs Optical Thermometer . . . . . . 53

32. Approximate Effect of Transmitted Optical Power on Measured Cutoff Wavelength, GaAs Temperature Transducer. . . . . . . . . . . . . . . . . 54

33. Components of First Prototype GaAs Temperature Sensor Prior to Assembly . . . . . . . 55

34. Coupling Element for Second Prototype Optical Temperature Sensor $\ldots \ldots \ldots \ldots \ldots \ldots$

35. Prototype Optical Temperature Sensor From Which Reported Wavelength-Temperature

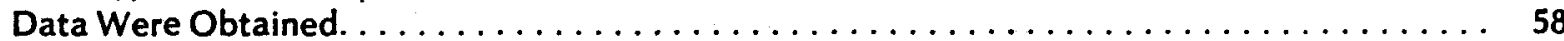

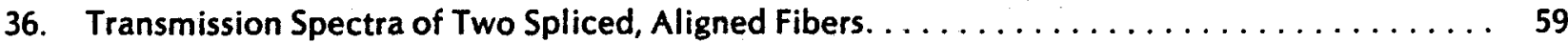

37. Effect of Axial Misalignment on Coupling Loss Between Two Butt-Spliced 5-mil Diameter Fibers . . . . . . . . . . . . . . . . . . . . . . . . . . . . . 60

38. Effect of End Separation on Coupling Loss Between Two Axially Aligned 5-mil Diameter Fibers 61

39. Principal Absorption and Emission Bands of $\mathrm{Nd}^{3+}: \mathrm{YAC} \ldots \ldots \ldots \ldots \ldots \ldots \ldots \ldots \ldots$

40. Schematic of Infrared Neutron Scintillation Detector Equipped With Schwarzchild Coupler to Fiber Optic Cable . . . . . . . . . . . . . . . . . . . . . . . . . .

41. Relative Light Output of $\mathrm{ZnS}$ (Cu) Phosphor with Temperature Showing Position of Main YAC Absorption.

42. Photomultiplier Signals from ZnS-Coated YAG Crystal Under Alpha-Particle Bombardment . . 70

43. Schematic of Two-Mirror Optical Coupler $\ldots \ldots \ldots \ldots \ldots \ldots \ldots \ldots \ldots \ldots \ldots \ldots \ldots \ldots$

44. Contour Plot of Light Rays Exiting From Coupler in Figure $\mathbf{4 3}$ for $\mathbf{3 0 0}$ Rays Emitted from Points Displaced Vertically and Horizontally from Ideal Source Position at " $a$ " . . . . . . . . . 
45. Experimental Arrangement for Investigating Schwarzchild Reflection Systems for

Coupling Extended Source to Optic Fiber

46. Normalized Output of GaAs Pn Led as a Function of Wavelength and Temperature. . . . . 76

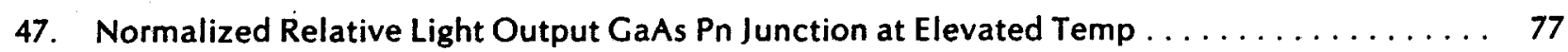

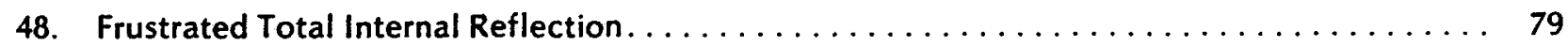

49. Electro-Optic Waveguide Modulator, Using $\mathrm{LiTaO}_{3} \mathrm{Slab} \ldots \ldots \ldots \ldots \ldots \ldots \ldots \ldots \ldots$

50. Pressure Transducer Employing Frustrated Total Internal Reflection $\ldots \ldots \ldots \ldots \ldots \ldots \ldots$

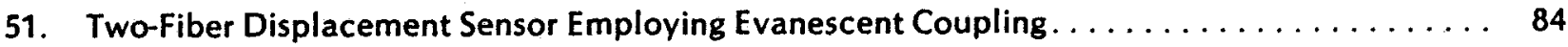

\section{APPENDIX A}

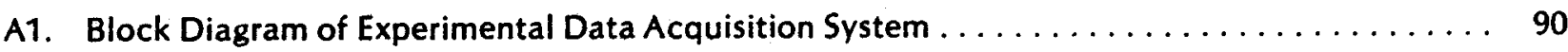

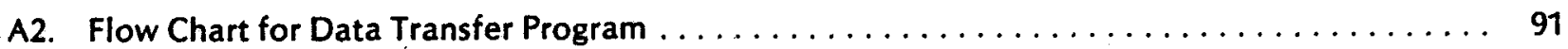

A3. Flow Chart for Multiplot Program $\ldots \ldots \ldots \ldots \ldots \ldots \ldots \ldots \ldots \ldots \ldots \ldots \ldots \ldots \ldots$

A4. Raw Data for Temperature Sensor Experiments Using the Original Gallium Arsenide Crystal. .. 93

A5. Reduced Data from Temperature Sensor Experiments Using the Original Callium

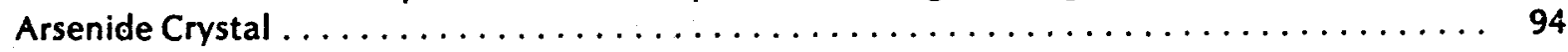

A6. Calibration Data for Temperature Sensor Experiments $\ldots \ldots \ldots \ldots \ldots \ldots \ldots \ldots \ldots \ldots$

A7. Measured Absorption Edge for GaAs as a Function of Temperature, Corrected For Reflection. . 96

A8. Absorption Coefficient of GaAs, Points of Maximum Slope with Temperature . . . . . . . 97

A9. Experimental Determination of Reflectivity as a Function of Wavelength for Various

Values of Temperature, GaAs. . . . . . . . . . . . . . . . . . . 99

A10. Steepness . . . . . . . . . . . . . . . . . . . . . . 102

\section{APPENDIX B}

B1. Ray-Trace Solution to Coupling Problem Between Optical Fiber-GaAs Wafer-Optical

Fiber System Including Variable Air Gap Between Exit Surfaces . . . . . . . . . . . . 104

B2. Schematic of Exact Solution to Case of Rays Exiting a Cylinder from Points Within the Cylinder 107

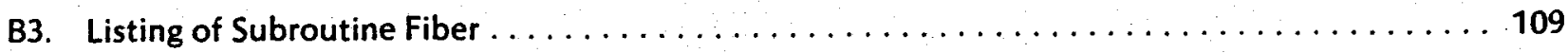

B4. Input/Output Data for Sample Problems Using Subroutine Fiber $\ldots \ldots \ldots \ldots \ldots \ldots \ldots \ldots$ 


\section{APPENDIX C}

C1. Two-Level Model for Radiative (R) and Non-Radiative (NR) Recombination in Semiconductors . 112

C2. Experimental Arrangement for Determining Temperature Effect on Emission of GaAs Led ... 114

C3. Output of GaAs Pn Led as a Function of Wavelength and Temperature $\ldots \ldots \ldots \ldots$

C4. Normalized Output of GaAs Pn Led as a Function of Wavelength and Temperature. . . . . . 117

C5. Total Integrated Light Output from a GaÁs Led as a Function of Absolute Temperature . . . . 118

C6. Position of Peak Output of GaAs Led as a Function of Temperature With Least Squares Fit to Data ........................................ 119 


\section{INTRODUCTION}

This report documents the results of a one-year program designed to investigate the feasibility of optical techniques applied to well-logging, to extend measurement capabilities in high-temperature geothermal bore holes. The basic concept is shown schematically in Figure 1. It makes use of a special armored cable containing fiber optic wave guides, connected to passive, downhole optical transducers. The latter modulate an optical carrier in response to bore-hole parameters. The optical carrier is a beam of infrared light transmitted from an optical source at the surface over an optical fiber. The modulated beam from the transducer is then returned to the surface over a second fiber, where conventional optical communications techniques are used to detect and decode the down-hole information.

Quartz, from which low-loss optical fibers are constructed, is virtually unaffected by temperatures at least as high as $600^{\circ} \mathrm{C}$ (the softening point of quartz is $1100^{\circ} \mathrm{C}$ ). The use of optical signal transmission in well-logging offers the possibility of bypassing many problems normally associated with high-temperature operation. Since optical fibers are highly efficient communication channels, optical communication also offers the capability of measurements which require higher data rates than conventional electrical cables permit. Since neither the optical fiber nor passive optical transducers require electrical power, problems associated with electronic devices at high-temperatures will not occur.

However, the implementation of such an optical system must overcome formidable obstacles. Quartz fibers themselves exhibit inherently high tensile strength, but their relatively low ductility and susceptability to brittle fracture, require new concepts in cable construction. Quartz also must be protected from direct contact with hot steam or water, requiring protective coatings which can tolerate the high temperatures encountered.

Geothermal energy offers one of the most immediately available energy options under current investigation. A serious limitation in the evaluation of this resource, lies in the inability to reliably make adequate geophysical measurements at those temperatures of greatest interest to successful geothermal exploitation. The optical approach offers one step along the path toward solving these difficulties.

\section{BACKGROUND}

\section{A. Geothermal Energy}

One of the alternate energy sources being seriously pursued in the United States and other parts of the world is the natural thermal energy in the earth's crust. The heat associated with increasing penetration into the earth is accentuated and its exploitation made practical by certain anomalous conditions occurring in specific areas of the world. While the sources of heat present complex questions open to controversy, it is now generally understood that most, if not all, of the areas exhibiting the greatest practical geothermal potential overlie intrusive masses which have penetrated strata relatively near the surface from deeper regions, during recent geologic time. The common exploitation concept now being pursued in the utilization of geothermal energy includes the drilling of wells into suitable rock strata overlying these intrusive masses. Such strata contain naturally occurring water which is subsequently produced at the surface as steam or super-heated liquid. Part of the heat contained in the fluid is transferred to a heat engine or other system either directly or by means of a heat exchanger. The spent thermal fluid is then disposed of, preferably by reinjection into the reservoir rock.

In order to be useful as a reservoir for thermal fluid, the rock must have suitable properties, including high temperature, contained water or steam, and sufficient porosity and permeability to permit the production of adequate quantities of fluid. In many cases, the porosity and permeability are provided by the complex fracturing of metamorphosed sedimentary rock. Research effort is also being expended on a method of hydraulically or otherwise fracturing nonpermeable rock, with the subsequent development of artificial hydrothermal reservoirs by means of water introduced into the fractures from the surface. 


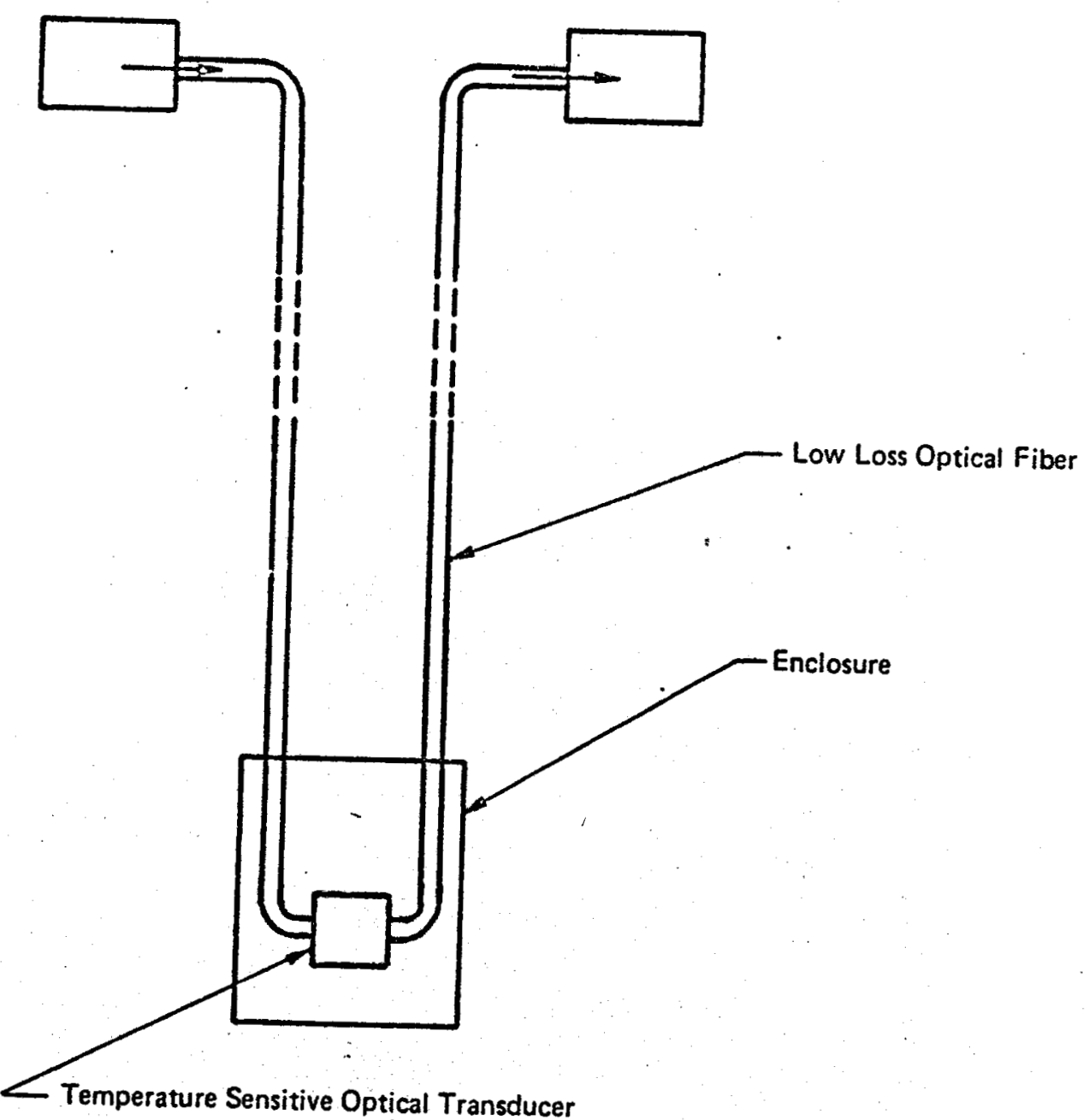

FIGURE 1. SCHEMA TIC OF THE BASIC OPTICAI LOGGING CONCEPT. . 


\section{B. Limitations of Down-Hole Measurement Systems}

Regardless of the type of geothermal reservoir involved, determination of the exact geological and geophysical parameters within such zones presents serious problems. Direct measurement of acoustical, electrical, thermal and other properties of such reservoirs in place, accomplished routinely in petroleum exploration, is generally prevented by the high temperatures encountered. Equipment used in making such measurements, normally referred to as "well-logging", is currently limited in operation to temperatures below $150^{\circ}$ to $200^{\circ} \mathrm{C}$, with the vast majority of petroleum exploration work not exceeding $100^{\circ} \mathrm{C}$. The main zones of interest for the recovery of geothermal energy are at temperatures above $250^{\circ} \mathrm{C}$. These temperatures are generally beyond the operating limits of existing commercial well-logging systems. Even at $200^{\circ} \mathrm{C}$, only limited measurements are possible and these are accomplished with great difficulty. Without suitable down-hole measurements, the knowledge of geothermal reservoir systems is severely handicapped; conditions must be inferred from cores, surface flow measurements and other indications which do not permit the establishment of accurate reservoir parameters. The latter are vital to effective exploration and the development of efficient, effective exploitation practices.

Well-logging, as understood within the petroleum industry, consists of the measurement of downhole geologic parameters in which an electrical cable positions an instrument package accurately within a bore-hole and transmits electrical signals to the surface in response to certain established measurements. The cable normally also supplies power to the instrument package, although the latter function is occasionally performed by self-contained batteries. Generally speaking, thermal, electrical, acoustic or nuclear properties of the rock are measured directly, and from these are inferred the lithology, rock porosity and fluid content. Other functions are frequently served as well, including fluid sampling, side-wall sampling, pressure measurement, and others.

\section{SYSTEM CONSIDERATIONS}

\section{A. Principles of Fiber Optics}

An optical fiber is a thin, flexible waveguide generally made of glass or plastic. Light which enters the fiber at one end follows a grazing path down the fiber, continually reflecting internally off the surface. The fact that the light fails to pass through the wall of the fiber and escape is due to a phenomenon known as "total internal reflection". So long as a light ray strikes the wall of the fiber at an angle less than the "critical angle", it cannot pass through, but is reflected back into the fiber.

The critical angle depends on the ratio of the indices of refraction of the two media at a boundary. In the case of glass and air, this ratio is about 1:1.5 and the critical angle, by Snell's Law, is

$$
\theta_{c}=\cos ^{-1}\left(\frac{n_{2}}{n_{1}}\right)=\cos ^{-1}(0.67)=48.2^{\circ}
$$

Fibers of the type conisidered in this program are "clad" fibers, in which the refractive indices of two different kinds of glass make up the internal reflection properties, with no dependence on the outside environment.

For a straight rod, the grazing angle is the same from one reflection to the next and depends on the angle at which the light enters the fiber. If a fiber is bent, the grazing angle is increased and the light loss will increase. However, low-loss fibers now available permit bend radii as small as a few $\mathrm{cm}$ without significant signal loss.

Bundles of optical fibers may be used to carry images. A good example of naturally occurring optical image transmission is the human retina. The assembly of rods and cones carries the image formed by the lens of the eye to a location where it is converted into the visual stimulus. 
Clad quartz fibers (known as "glass on glass") are drawn in a flame or laser beam from glass rods. The fibers can have diameters from less than 2 micrometers (about two wavelengths of visible light) to several hundred micrometers. Continuous lengths of clad quartz fibers one $\mathrm{km}$ or more in length are now routinely produced.

Fiber optic transmission lines are potentially inexpensive. Current costs, largely dependent upon demand, are in the range of $\$ 1.00$ to $\$ 6.00$ per meter, and are expected to go as low as ten cents per meter for large quantities. They are not electrically conducting so that ground loops, short circuits, and leakage problems are not possible.

Electromagnetic interference and susceptibility is not a problem, and glass fibers can exhibit tensile strengths as high as 600,000 psi. Optical fibers of the type considered in this program are small (diameters less than $\mathbf{2 0 0}$ micrometers) and light weight. The bandwidth capacity of an optical fiber transmission line is several orders of magnitude greater than electrical coaxial conductors, and transmission losses of optical fibers can be as low as or lower than $I^{2} R$ losses for comparable lengths of wire. Because of the small size of individual optical fibers, a number of them can be built into a cable with a small diameter. Since the enormous bandwidth of an optical fiber generally eliminates the need for multiple signalpaths, redundancy and convenience in deployment of optical paths are possible. In fact, even without the temperature considerations, optical transmission lines have considerable merit for well-logging purposes in their own right. A typical commercial quartz fiber is shown in Figure 2.

\section{B. Status of Fiber Optics Technology}

In 1974, the Naval Electronics Laboratory Center (NELC) at San Diego, California obtained a $3 \mathrm{~km}$ length of quartz optical waveguide from the Corning Class Works, exhibiting attenuation of $2.1 \mathrm{~dB} / \mathrm{km}$. The delivery culminated a long, intense effort to develop a very low-loss optical fiber in relatively long lengths. This fiber has been followed in rapid succession by others of improved characteristics and by 1977 several manufacturers have announced production of fibers in $1 \mathrm{~km}$ lengths, exhibiting attenuation of $1 \mathrm{~dB} / \mathrm{km}$ or less. Losses of this order are low enough to permit cables of $10 \mathrm{~km}$ length or more without repeaters.

Optical fibers are now routinely incorporated into transmission cables for various purposes. Under sponsorship of NELC and Army Electronics System Command, both high-pressure underwater cables, and cables suitable for Army field operation have been developed and are commercially available. Cables for high-temperature operation have not been reported however, and in fact, the subject of temperature effects on fibers had only been reported in a cursory fashion prior to this program.

Tensile strength along with optical attenuation of optical fibers has been the subject of continuing improvement. Clad fibers with tensile strengths as high as 200,000 psi are now commercially available. This is sufficiently strong to permit an optical fiber to support its own weight over a vertical length of more than $20 \mathrm{~km}$.

The longest continuous fiber reported to date is $5 \mathrm{~km}$ in length; however, there appears to be no fundamental reason why longer fibers cannot be produced. An acceptable alternative to the manufacture of very long fibers is splicing. Losses less than $1 / 2 \mathrm{~dB} /$ splice have been reported by fusing quartz fibers.

Connecting fibers to each other and to other components without fusing, presents difficult practical problems. Considerable effort has been devoted to the development of optical connectors for single fibers, with some success. The subject is by no means closed, however, and it was recognized in the early stages of this program that the design of a satisfactory cable-head connector for a fiber optic logging system would present formidable problems, particularly under the conditions of high temperature and high pressure. Detachable connections for military field equipment have been reported which exhibit 
v

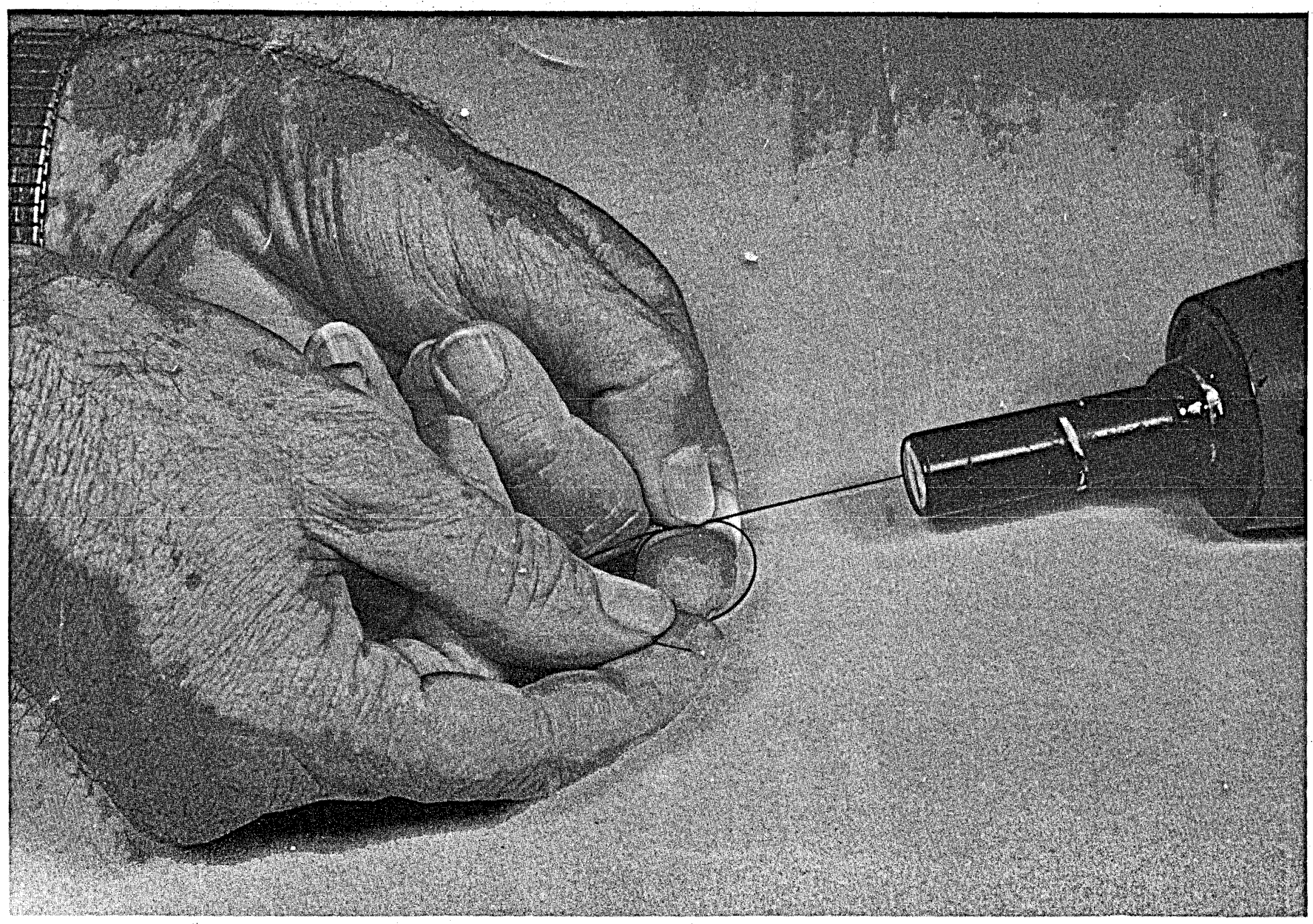

FIGURE 2. CLAD QUARTZ OPTICAL FIBER ILLUMINATED BY MICROSCOPE LAMP The fiber is in a very tight bend around the holder's finger. 
individual losses of about $1.5 \mathrm{~dB} /$ connection; however, in practice it is difficult to achieve repeatable performance of this level. Even then, preparation of the fibers is tedious, and connector assembly accommodates itself to field operation with great difficulty. An ideal solution to the connector problem is still being sought.

\section{Optical Sources}

Practical fiber optic communication systems consist of other elements in addition to the optical transmission line. These include optical sources, modulators, detectors, and the accompanying hardware to couple light into and out of the fiber. Practical sources for those fiber optic systems now in use or contemplated can be divided into two groups: (1) semiconductors, including light emitting diodes and laser diodes and (2) lasers, generally the neodymium-doped solid-state variety, but also gas lasers of various types. The wavelengths of these two groups of sources are usually chosen to coincide with the optimum transmission wavelengths of quartz fibers, between 0.80 and $1.1 \mu \mathrm{m}$ (a narrow band of wavelengths near $0.95 \mu \mathrm{m}$ should be avoided, as there is a strong absorption in quartz near that wavelength). Neodymiumdoped solid-state lasers normally emit at $1.06 \mu \mathrm{m}$, very nearly ideal.

Semiconductor sources are directly modulated via their drive currents. Light emitting diodes are operated CW and their modulation is usually analog. Laser diodes, at room temperature, are pulsed in order to reduce thermal problems. They lend themselves to pulsed modulation techniques such as PPM or PCM.

For higher applications, Nd:YAC lasers are used, although they cannot be directly modulated. For well logging these lasers might be advantageously used, since the optical source is expected to be at the surface, and the modulator down-hole.

Two general classes of modulators may be considered: the first includes mechanical devices such as choppers, tuning forks, and moving mirrors, and the second the electro-optic, magneto- optic, acoustooptic and absorptive type devices. Mechanical modulators might operate at temperatures as high as $300^{\circ}$ to $400^{\circ} \mathrm{C}$, and could be powered from a mechanical storage device such as a spring. The electro-optic and acousto-optic modulators can achieve much greater bandwidth, at least $1 \mathrm{MHz}$ for 1 to $5 \mathrm{~mW} / \mathrm{MHz}$ of modulating power. Magneto-optic devices at the wavelengths being considered for this application are not readily available, and absorptive type modulators have not been well investigated. Thus, it appears that electro-optic or acousto-optic modulators are more suitable for down-hole applications. Specific modulator approaches are discussed in a later section of this report.

Special requirements generated by one of the down-hole optical sensors investigated in this program, makes mandatory the consideration of a fourth type of optical source. A passive optical temperature transducer has been developed in which the absorption edge, or cut-off wavelength, of a semiconductor is measured as a function of temperature. This requires either a broad-band, or a tunable optical source. Broadband sources are not generally required in optical communication systems and the subject is not well covered in the open literature. Ordinary incandescent lamps appear to fill this requirement, although not ideally. Tunable sources are generally restricted to the "dye lasers". Unfortunately, dye lasers are not currently available over the wavelength range of interest in this application.

A second "tunable" possibility is a semiconductor LED with temperature control. A properly doped semiconductor, operated so that its operating temperature could be carefully controlled, could be made to produce an output of variable wavelength.

\section{Optical Detectors}

Detectors for optical well-logging requirements may be divided into two groups: (1) semiconductor detectors and (2) photomultipliers. Semiconductor detectors can be further subdivided into avalanche 
detectors and non-avalanche detectors. Photomultipliers are physically less desirable than semiconductors, but can develop greatly improved signal-to-noise performance at high sensitivity. In the system philosophy developed during this program, the detection operation would be accomplished at the surface rather than down-hole and, consequently, the difficulty of photomultiplier operation is greatly reduced and poses only minor problems. The specific choice of a photodetector depends upon the final system parameters, including bandwidth, attenuation, and available signal power.

Coupling into and out of fibers is a critical task. A rather extensive literature search has been devoted to the subject of coupling, the main problem being the narrow acceptance angle and small size of optical fibers. Because of the narrow output beam, laser sources are inherently capable of much higher coupling efficiency than any other sources available. Collection optics and other aids to coupling the output of extended sources in the fibers has been the subject of a number of papers. ${ }^{(1-4)}$

\section{CABLE DESIGN AND FABRICATION}

Obviously, one of the most significant elements in the optical well-logging system is an optical cable. Because well-logging is performed in a very severe environment, conventional electrical logging cables are typically of a steel-armored type, in which a double layer of steel stress strands overlays a central core containing several insulated electrical conductors. In the concept explored in this program, the intent is to replace the electrical conductors with optical fibers. These require no electrical insulation, and are in themselves inherently insensitive to temperature effects.

Optical cables of various types, including high-pressure, under-sea cables, have been constructed in the recent past but none designed for high-temperature operation. A number of practical problems associated with combining optical fibers into a well-logging cable were found to exist. It is believed that these have now been identified, and can be attacked in a systematic way.

\section{A. Subcontractor Selection}

Early in the program, well-logging cable manufacturers were solicited for proposals to construct a $100 \mathrm{M}$ length of optical cable for demonstration purposes, with the following general requirements:

- Two or more low-loss optical fibers, armored for well-logging operations.

- Optical attenuation of $15 \mathrm{~dB} / \mathrm{km}$ or less

- Temperature capability as high as possible, with a design goal of $300^{\circ} \mathrm{C}$.

- A design capable of eventual extension to lengths of at least $10,000 \mathrm{ft}$.

The three manufacturers solicited were the Rochester Corporation, ITT-Electrooptics, and Vector Cable Company. All of the companies expressed interest, and two furnished specific proposals.

The Rochester Corporation was at that time working in conjunction with Corning Glass Works, in an attempt to armor a Corning low-temperature commercial optical cable known as "Corguide". Rochester furnished a sample of this cable, shown in figure 3 . Their attempts to produce the cable in useful lengths were unsucessful, however, and the company declined to make a specific proposal in answer to our request.

ITT-Electrooptics Division, in conjunction with ITT Hydrospace Division, proposed to design and fabricate a cable to meet our general specifications, utilizing TFE as the buffer material. Their cost estimate for this development was outside the scope of the present project and, as a consequence, the proposal was forwarded to the ERDA Project Manager with the recommendation that it be funded separately. 
$\infty$

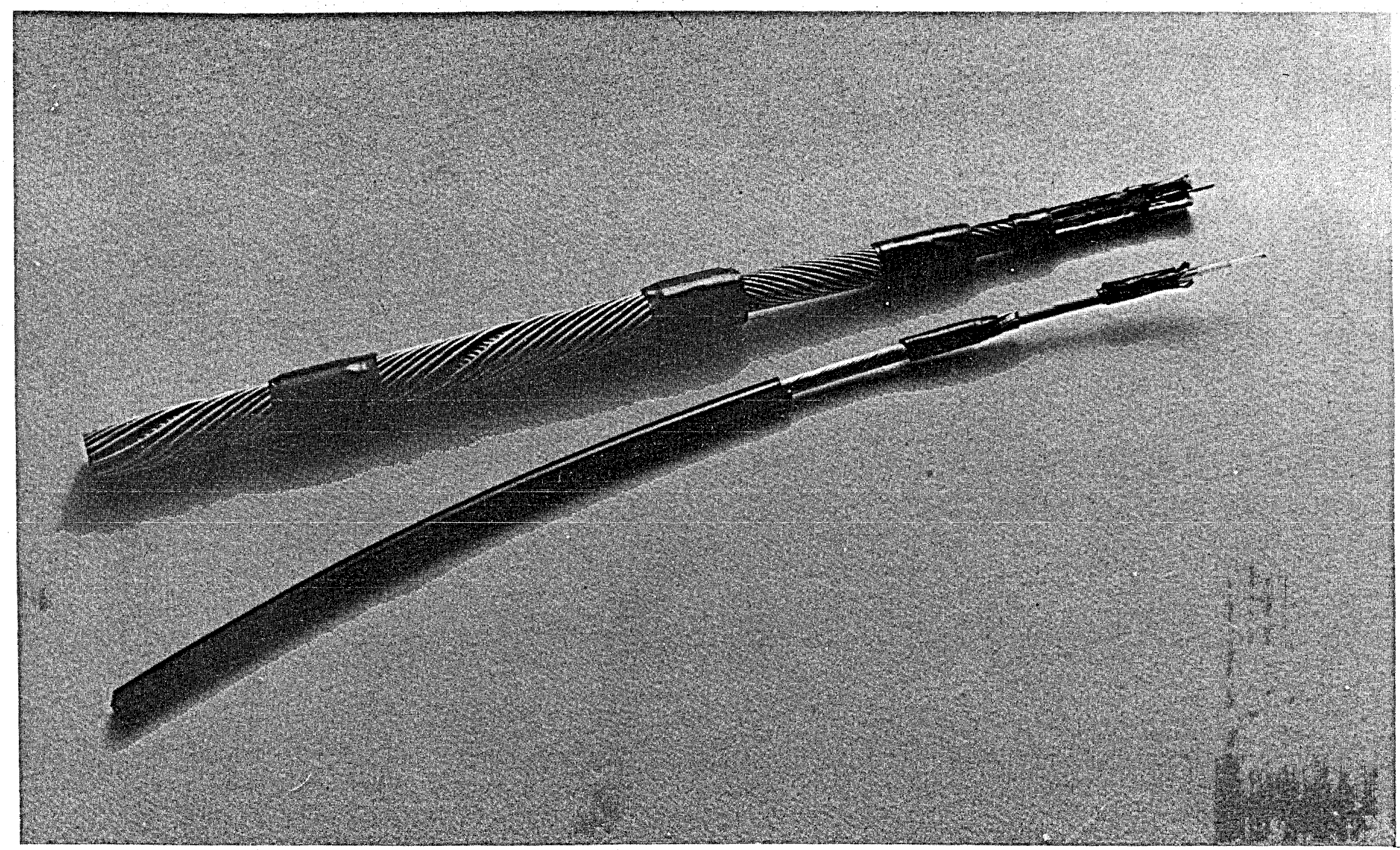

FIGURE 3. SAMPLES OF LOW-TEMPER ATURE ARMORED FIBER -OPTIC CABLES. The larger sample contains seven optical waveguides, but manufacture was not entirely successful. (Rochester Cable Co.) 
Vector Cable Company, the largest supplier of well-logging cable, agreed to undertake the development and provide a demonstration cable on a procurement basis. This proposal, which was within the financial capability of the contract, was accepted and all project information concerning optical fibers and optical cables was furnished to Vector. Consequently, the principal report of cable development concerns the work of Vector and its fiber supplier, Times Fiber Communications, Inc.

\section{B. Design Considerations}

A well-logging cable is primarily for the purpose of transmitting power and signals, but it also must serve as a strong, precision "measuring tape" and hoisting element as well. Since well parameter correlation with depth is based on cable measurement, particular stress is placed on the physical stability of the cable, and on its behavior under load. Currently, these goals are accomplished by using steel stress members for which the stress-strain properties are predictable. Stranded copper conductors are typically used in the electrical circuits, and steel armor strands provide the mechanical strength and load carrying capability. Copper, with its high ductility, can easily conform to the elongation requirements dictated by the steel armor.

In constructing an armored optical cable, a serious limitation is introduced by the properties of the quartz fiber. Quartz under normal temperatures is not a ductile material and while exhibiting great tensile strength, it is subject to fracture with very low elongation. Moreover, the high sensitivity of optical fibers to microbend stresses, places severe restrictions on the action of the other cable members. The very small size of these fibers makes them relatively fragile in spite of the excellent tensile properties of the material.

An initial design study of the cable requirements, both by Vector and by the SwRI project team, disclosed three general conceptual approaches to armored cable design with fiber optics.

\section{Conventional Double-Armor over Optical Core}

In the approach taken by Rochester and Corning in the cable design illustrated previously in Figure 3 , an inner core containing the buffered fibers is armored in conventional fashion. The optical core contains two stress strands of aramid yarn. Aramid has elongation properties similar to those of glass, but with high fracture toughness. The design is based on the idea that tensile forces will be absorbed by the inner stress strands, protecting the individual optical fibers. Rochester found this approach unsatisfactory.

\section{Conventional Construction, with Glass Armor}

Presumably, a design based on glass yarn strands as the protective armor might be feasible. While such an approach may have long range significance, it is beyond the scope of existing logging cable technology. Adequate protection of the glass armor from hot well fluids also presents problems.

\section{Fexible Tube}

The optical fibers might be isolated inside a flexible tubular structure in the cable core, the tube protected by conventional steel armor. While this approach is appealing, construction of such a tube with sufficient flexibility, strength, and mechanical stability, presents severe manufacturing problems. Consideration was given to this concept in the program, but without a promising design approach.

\section{Stress Member Inside, Non-Load Bearing Armor Outside}

Vector recommended this approach with the concurrence of the project design team. The Vector cable design based on this concept is shown in Figure 4, in which a central steel stress member carries the 


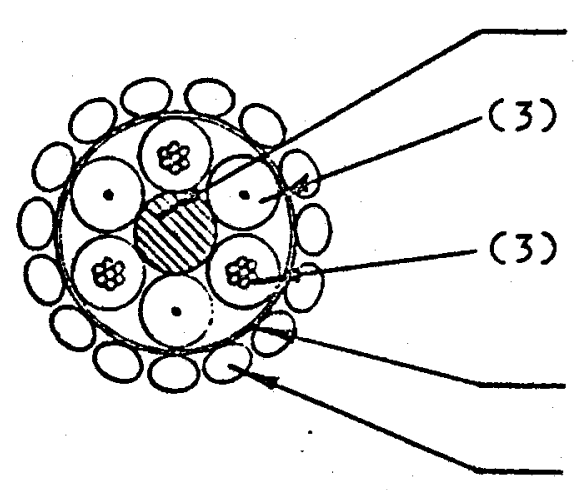

STRESS CORE $.079^{\prime \prime}$ GIPS O.D. $=.079^{\prime \prime}$

OPTICAL FIBERS WITH PFA JACKET

$O . D .=.068^{\prime \prime}$

ELECTRICAL CONDUCTORS WITH .015"

PFA INSULATION $0 . D_{0}=.068^{\prime \prime}$

BINDER TFE, GLASS TAPE O.D. $=.223^{\prime \prime}$

SINGLE ARMOR, 15-.031" GIPS

$54^{\circ}$ ANGLE O.D. $=.285^{\prime \prime}$

Specifications:

ELECTRICAL PROPERTIES;

DC RES ISTANCE;

$11.0 \mathrm{OHMS} / 1000^{\prime} 220^{\circ} \mathrm{C}$.

VOLTAGE RATING:

INSULATION RESISTANCE:

23.2 OHMS $/ 1000^{\prime} \theta 300^{\circ} \mathrm{C}$.

500 VRMS

2000 MEGOHMS $/ 1000^{\circ}$ (12 $20^{\circ} \mathrm{C}$.

$.1 \mathrm{MEGOHMS} / 1000^{\circ}$ \& $300^{\circ} \mathrm{C}$.

OPTICAL PROPERTIES:

OPT I CAL PATH:

$1.08623^{\circ}$ ANGLE

MECHANICAL PROPERTIES:

BREAK STRENGTH:

WEIGHT IN AIR

$1210 \#$

TEMPERATURE RATING:

$105.4 \# / 1000^{\circ}$

$300^{\circ} \mathrm{C}$

FIGURE 4. TENTATIVE HIGH-TEMPERA TURE CABLE DESIGN, COMBINING THREE OP TICAI AND THREE ELEC TRICAL PATHS. (Vector Cable Co.) 
full cable load. Class optical fibers, heavily buffered with a resilient buffer material, surround the central stress core in a loose helix. This core is bound with glass tape, and a single protective non-load bearing armor wound over it. Under load, the central stress member tends to stretch, and the buffered optical fibers accommodate by "unwinding" slightly from their no-load position. The armor strands follow, tending to unwind also. Thus, under load there is a gentle "spring" action, with a return to the initial position as the load is reduced. The design calls for a 0.079 -in. steel core surrounded by three optical fibers and three electrical conductors, all with diameter 0.068 -in. $(1.71 \mathrm{~mm})$ over the buffer. The outside diameter of the complete cable is $0.285 \mathrm{in}$. $(7.3 \mathrm{~mm})$.

\section{Optical Fiber Considerations}

\section{Selection of Optical Fibers}

Because the lengths of cable used in well-logging are relatively great, the use of low-loss optical fibers (less than $15 \mathrm{~dB} / \mathrm{km}$ ) is dictated. Fibers with this capability are constructed of quartz, with either a step or graded-index quartz cladding. Graded index fibers are employed where very high bandwidths are required, and since bandwidth greater than $5 \mathrm{MHz}$ is easily attainable by lower-cost step-index fibers, the latter are adequate for any well-logging purpose now envisioned. Graded index fibers can be substituted in the design at any later time should they appear necessary.

The two development areas presently receiving the greatest attention in the fiber optics industry, concern fiber strength and reduced attenuation. Improvements in both these parameters are announced frequently, and the availability of fibers with tensile strengths greater than 200,000 psi and with attenuation in the range of $1 \mathrm{~dB} / \mathrm{km}$ or less are assured for the near future. For the purposes of this program, fiber strength was determined to be of greatest concern for the demonstration phase of the program. High strength fibers with attenuation below $15 \mathrm{~dB} / \mathrm{km}$ are readily available, and were deemed suitable for use in the short length demonstration phase of the program. Consequently, the design goal for the prototype cable was set at $15 \mathrm{~dB} / \mathrm{km}$ or less, with fiber strength the overriding concern. Substitution of fibers with lower attenuation can be accomplished in the cable design at any time, once the feasibility has. been demonstrated.

\section{Fiber Buffering}

While optical fibers need not be electrically insulated, it is essential that they be protected by a resilient coating called a "buffer coat", to minimize the effects of microbends. Microbends are uneven stress distributions in microscopic areas of the cladding. These affect the index of refraction slightly, and cause localized zones of increased loss. The individual effect of a single microbend is insignificant. Over a long length, however, the cumulative effect can be serious. A relatively soft, resilient coating over the fiber tends to equalize externally applied stresses, and protects the fiber from internal stress gradients.

A second purpose is served by the buffer coat. Super-heated steam or hot water is corrosive to quartz, and it is important that the fibers be isolated from direct contact with such fluids. The buffer material then must serve as an isolation barrier. Consequently, selection of an appropriate buffer material impermeable to water is an important part of the cable design. Typically, buffer coatings of various plastics are applied to the fibers at the time of manufacture. The fiber is first lubricated with a silicone oil, and the plastic extruded directly over it, forming an unbonded, tight-fitting continuous tube. If the buffer material is directly bonded to the glass, differential temperature expansion coefficients are likely to generate even greater stresses within the fiber. The results of such thermal expansion differences are microbends, severe losses, and ultimately, breakage of the fiber.

After extensive investigation of materials and manufacturing problems, two candidate buffer materials were selected as best fulfilling the buffer requirements. These materials are both fluoroplastics 
of the Teflon family, the preferred one of which is a relatively new compound known as PFA. (perfluoroalkoxy), and the other TFE (polytetrafluoroethylene). PFA is currently used in the manufacture of commercial quartz fibers, but in very thin coatings. It adapts readily to standard ram extrusion machines and has a service temperature near $275^{\circ} \mathrm{C}$. TFE, which has a slightly higher temperature capability, is not directly amenable to extrusion. It is generally applied in an aqueous dispersion as a paste, which must then be sintered in a separate process. After consultation with various fiber manufacturers, Vector recommended PFA as the most practical candidate for the prototype cable.

For eventual extension of the concept to temperatures of $300^{\circ} \mathrm{C}$ and higher, less certain buffer candidates appear. TFE, if methods for its application can be developed, should be useable to $300^{\circ} \mathrm{C}$. Higher temperature compounds, relatively new in the plastics industry, include the polyimides, paralene (a proprietary conformal coating) and metallic coatings such as electroless nickel. All these materials received consideration during the program, but the development of methods of applying them satisfactorily to optical fibers for operation over a wide temperature range will require a considerable research and development effort.

\section{Stress and Temperature Effects on Optical Fibers}

Investigation of the optical performance of quartz fibers at high temperatures and under mechanical stress, are subjects not well reported in the open literature. Consequently, during the program methods of determining these effects were developed and experiments conducted to assess the effects of stress and temperature.

Increased optical losses under tensile and other loads anticipated in a well-logging cable, are the result of microbends, or localized stress gradients in the quartz. The seriousness of these effects is primarily a function of the relatively long lengths of fiber required. Determining the effects in the laboratory, poses serious instrumentation problems. Practicality demands that the measurements be made on short lengths of fiber, but the magnitude of the losses over a short length is likely to slight. For example, a quartz fiber with an optical attenuation of $15 \mathrm{~dB} / \mathrm{km}$ will exhibit attenuation of only $0.15 \mathrm{~dB}$ over a $10-\mathrm{m}$ length. Sufficient accuracy to measure, in absolute terms, a $1 \mathrm{~dB} / \mathrm{km}$ change in attenuation would require measurement accuracy of $0.001 \mathrm{~dB}$. This is beyond the state-of-the-art of optical capability. In order to circumvent this difficulty, a method was selected in which a differential measurement between two identical fibers of the same length is made.

In this procedure both fibers are illuminated by a common extended light source, and the experimental stress state is applied to only one of them. Differences in the relative attenuation become readily apparent, and measurement accuracy of $0.1 \%$ can easily be achieved. This is equivalent to a relative change of $1 \mathrm{~dB} / \mathrm{km}$. A schematic representation of the apparatus used in this technique is shown in Figure 5.

There are four optical parameters in this measurement system which must be carefully controlled: a. fiber end preparation, b. source brightness, c. input launching (coupling) conditions, and d. output receiving conditions. These parameters must be uniform and repeatable from measurement to measurement, and time invariant if meaningful loss data is to be obtained.

Typical attenuation effects on a low-loss fiber subjected to pure tensile load are shown in Figure 6, in which the increase in attenuation (\%) is plotted as a function of tensile stress and \% elongation. The results indicate that, at an elongation of approximately $1 \%$, the particular fiber investigated showed an increase in attenuation of approximately $5 \%$. Thus, if $1 \mathrm{~km}$ of this fiber with an initial attenuation of $15 \mathrm{~dB}$ is subjected to a tensile stress resulting in $1 \%$ elongation (approximately $90 \mathrm{ksi}$ ), the fiber attenuation could be expected to increase $5 \%$, or to a total of $15.75 \mathrm{~dB}$, a relatively insignificant increase. 


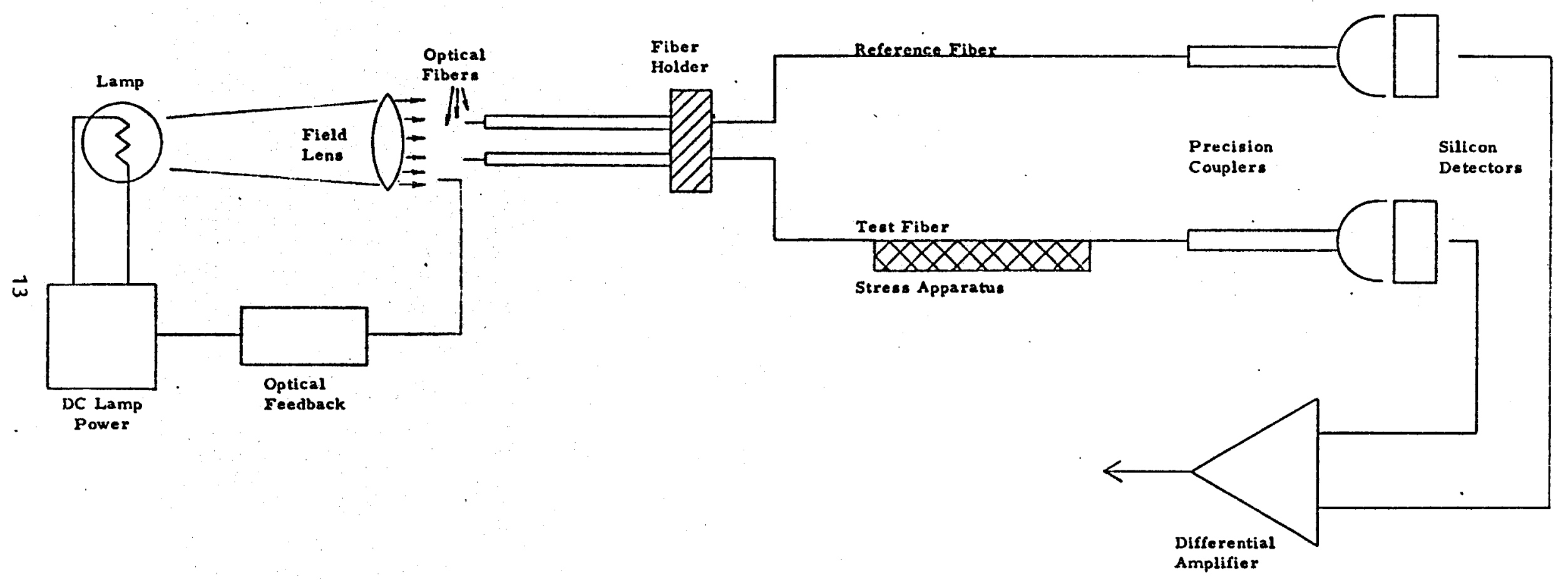

FIGURE 5. SCHEMATIC OF APPARATUS FOR DETERMINATION OF OPTICAL TRANSMISSION PROPERTIES OF SHORT FIBERS BY DIFFERENTIAL MEASUREMENT. 
C ".*: $4{ }^{\circ} 02$

FIGURE 6 : EFFECT OF TENSILE STRESS. ON ATTEHUATIOH

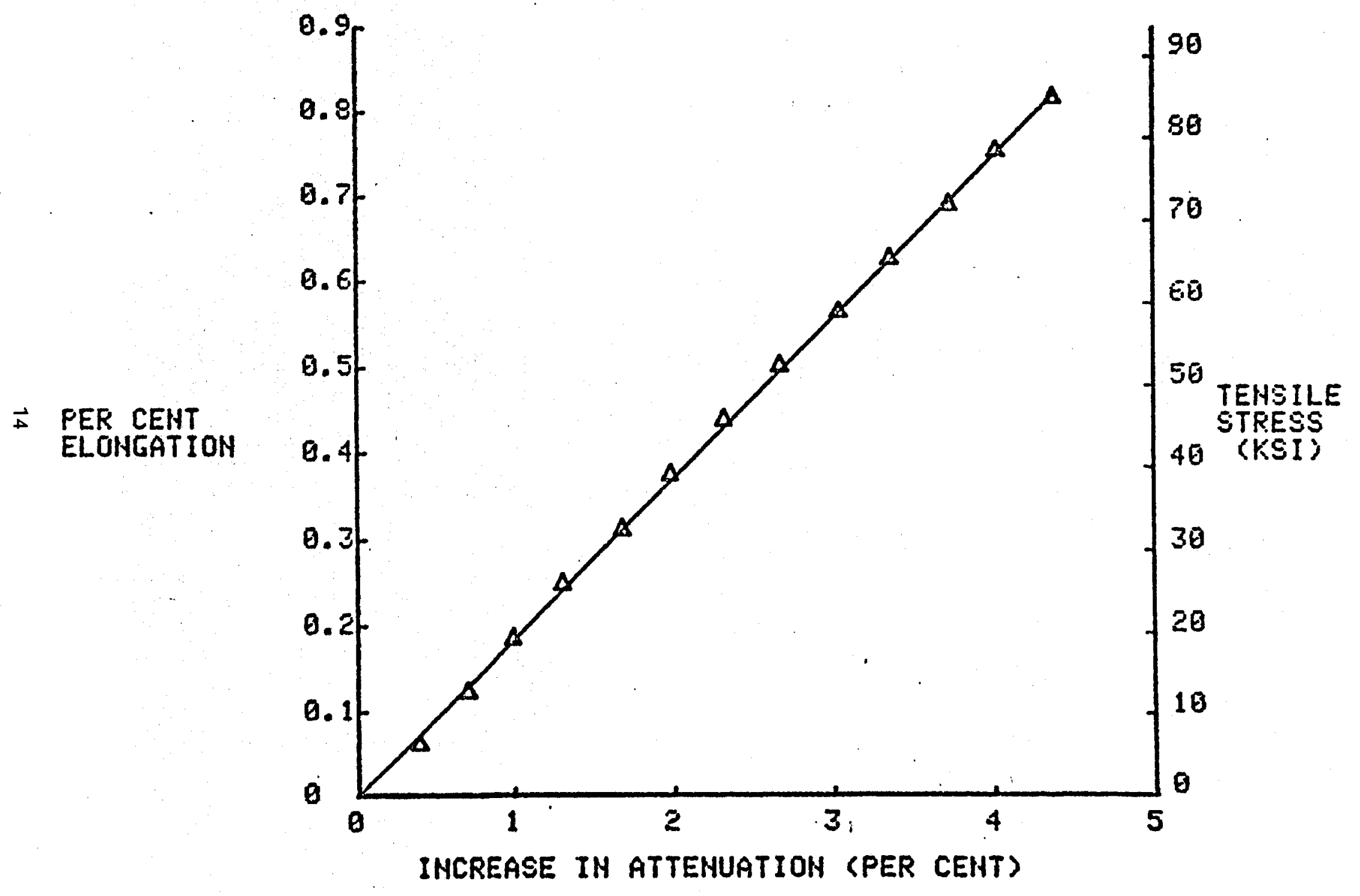


Temperature effect on attenuation was also investigated in the same manner, with the test fiber supported in a temperature test chamber. At $500^{\circ} \mathrm{C}$ no measurable effect on transmission of the heated fiber was detected. We conclude, therefore, that direct effects of temperature on attenuation can be expected to be less than $0.1 \%$.

Temperature can, however, be expected to affect the mechanical properties of the fiber. While no experimental determination was attempted in this program, the subject is well covered in the literature. At $400^{\circ} \mathrm{C}$, for example, quartz has a tensile strength approximately $70 \%$ as great as its room-temperature value.

\section{Cable Construction}

\section{Fiber Buffer Operation}

The heart of the Vector optical cable design is a step-index, low-loss fiber imbedded in a heavy buffer coating of PFA. Normally, buffer coatings on commercial fibers are very thin (on the order of $0.03 \mathrm{~mm}$ ). The initial approach to the buffering operation was to procure a standard commercial fiber buffered with PFA, and run it through a second extrusion process, to deposit a heavy layer of PFA over the first. Vector selected a fiber supplier, Times Fiber Communications, Inc., who agreed to meet the fiber specifications and to work with Vector on the buffering problem.

Initial attempts to produce the heavy PFA buffer coating showed that it would be necessary to make at least two additional passes through the extruder in order to maintain the fiber concentric inside the buffer. Difficulties were quickly encountered. The heavy PFA extrusion, with its relatively high mass and heat content, tended to melt the buffer coating and cause sticking. This introduced stresses into the fiber that resulted in breakage. The extrusion problem constitutes the basic difficulty of the method for which adequate solutions are still being sought. Vector worked at the problem for a period of several months even to the extent of obtaining uncoated fibers and attempting to extrude directly over them. Short lengths of apparently successful material were produced in this manner, but with the mass of PFA and the high extrusion temperatures, destruction of the silicone coating occurred frequently, with resultant wetting of the quartz and severe microbends. Finally, Vector called on Times Fiber to produce the complete buffered fiber directly, and supply it to Vector for the cabling operation.

Recognizing the fundamental problem of PFA sticking to the fiber, Times approached it by extruding an intermediate layer of a second material (an elastomeric nitrile rubber) to isolate the PFA from the glass. This procedure has met with limited success. At the time of this report, work is continuing on this approach. In the near future, it is anticipated that fibers will be delivered, and cable construction attempted.

\section{Summary of Results of Cable Investigation}

The difficulty experienced in producing a smooth, uniformly buffered fiber with sufficient buffer thickness to isolate the glass fiber itself, has constrained a proper evaluation of the cable design. The problem is principally associated with the high temperature at which the PFA extrusion is accomplished. In retrospect, a better approach to the optical cable evaluation would have started with construction of a low-temperature cable from which structural problems and performance could be assessed, with gradual improvement in the temperature capability sought in a systematic, step-by-step development program. 


\section{CABLE HEAD DESIGN}

In well-logging, it is convenient to have the cable terminated in a quick disconnect element known as a cable head, so that logging instruments can be easily changed. The requirements for such a connector on an optical cable include the following:

1. It must transfer the weight of the logging instrument to the cable stress member.

2. It must connect individual optical fibers in the cable to corresponding fibers in the instrument.

3. It should terminate the cable stress member in a "pull-out link", to permit emergency pull-off without leaving cable in the hole.

4. It must seal the individual fibers and their connections from well bore fluid under high pressure.

General investigation of cable head designs suitable for interfacing optical well-logging instruments to optical cable was carried out and a specific design developed for evaluation. Obviously, the failure to produce an optical cable hampered this evaluation seriously. Various aspects of the cable head design are discussed in the following paragraphs.

\section{A. Optical Connectors}

The large-scale application of fiber optics to routine communication applications has been slowed by the lack of a simple, reliable connector concept. The subject is receiving continued intensive effort on several technical fronts. This difficulty should not be considered surprising, in view of the fact that even electrical connectors have been the source of chronic problems in the electronic industry for decades. Some features of the electrical connection problem are common to optical connectors, in addition to which some new problems are posed.

Several manufacturers now commercially produce, on a limited basis, single fiber connectors. These have been only marginally successful. The conventional approaches to the connector problem can generally be catagorized as follows:

\section{Ferrule Type}

This is the most common approach to single-fiber connection. It involves cementing a fiber, generally stripped of its buffer coating, into a precision sleeve. The end of the fiber-ferrule assembly is then optically polished, and two similarly prepared fibers are matched into a mating sleeve in a precision butt joint. Extremely close dimensional tolerances are required of all connector components.

\section{Optical Lens Coupling}

In this concept, each of the two mating fibers is cemented to a small lens. The fiber-lens assembly is then inserted into a precision sleeve, the lens effectively increasing the coupling diameter. The system does not completely alleviate the alignment problem, however, since the fiber and the lens must be aligned with great accuracy.

\section{V-Groove Approach}

Precision V-shaped grooves are milled into a plastic or metal block and fibers cemented into individual grooves. Two such assemblies are then mated and aligned with precision pins. 
These three approaches seek to solve the fundamental problem involved in coupling two clad fibers, namely excessive optical loss due to misalignment. Losses at such a connection are the result of three separate positioning problems: lateral displacement, resulting from imperfect flush mating of two fiber ends; axial misalignment, due to dimensional variations in the fibers and in the ferrule or V-groove; and angular misalignment, or lack of parallelism between the two halves of the assembly.

\section{B. Investigation of Losses Due to Coupling Errors}

During this program, an assessment of the various sources of error was made by computer model and by experimental verification. These two approaches are discussed more fully in another section of this report (Section VI.A.7, "Prototype Temperature Sensor Design and Fabrication", and in Appendix B, "Program OPDEAN"). In summary, the results indicate that two 5 -mil $(0.13 \mathrm{~mm})$ step-index fibers joined in an otherwise perfect butt splice, exhibit a loss of $2 \mathrm{~dB}$ with only 1 -mil $(0.03 \mathrm{~mm})$ axial misalignment. Since there are inherent errors in both the OD of the fiber and of the position of the core within the fiber, the dimensional requirements for alignment hardware such as ferrules or sleeves, are extremely severe. By comparison, fiber separation along the axis under the same conditions (in air), produces an equivalent $2 \mathrm{~dB}$ error for approximately a $10-\mathrm{mil}(0.25 \mathrm{~mm})$ separation distance, representing $200 \%$ of the fiber diameter.

\section{Design Concept}

In view of the importance of accurate axial alignment in coupling optical fibers, a connector design was undertaken for the optical cable head, in which alignment accuracy was a paramount consideration. For well-logging this alignment requirement is made even more stringent by the necessity of connecting several fibers simultaneously. Based on the combined problems of optical considerations, high pressure, and the severity of field operation, a concept was developed with the following principal features:

- A conventional pressure housing and rope socket.

- Pressure seals external to the optical connector proper.

- An optical connector separate from, but secured within the pressure housing itself.

- Very accurate individual fiber alignment.

\section{Pressure Connector Design}

A simplified assembly of the complete optical cable head design is shown in Figure 7 , in which the major components of the assembly are shown.

\section{Fiber Optic Connector}

At the heart of the cable head is the small fiber optic connector, held inside the pressure housing (shown at $A$ in Figure 7). The basis for this concept is alignment of the fibers by means of an assembly composed of a series of small chemically milled plates. These serve as alignment fixtures for precision ferrules, each of which holds one of the three optical fibers in the cable. A detail of one half of this connector assembly is shown in Figure 8 . The mating assembly is identical except that it contains holes for the alignment pins. Each connector half holds the three ferrules and individual fibers. The chemically milled disks can be produced to extremely close tolerances (less than $0.002 \mathrm{~mm}$ ) and consequently are more accurate for alignment purposes than machined parts of comparable complexity.

In the assembly, each of the three stripped fiber ends is inserted into one of the slotted sleeves, and 


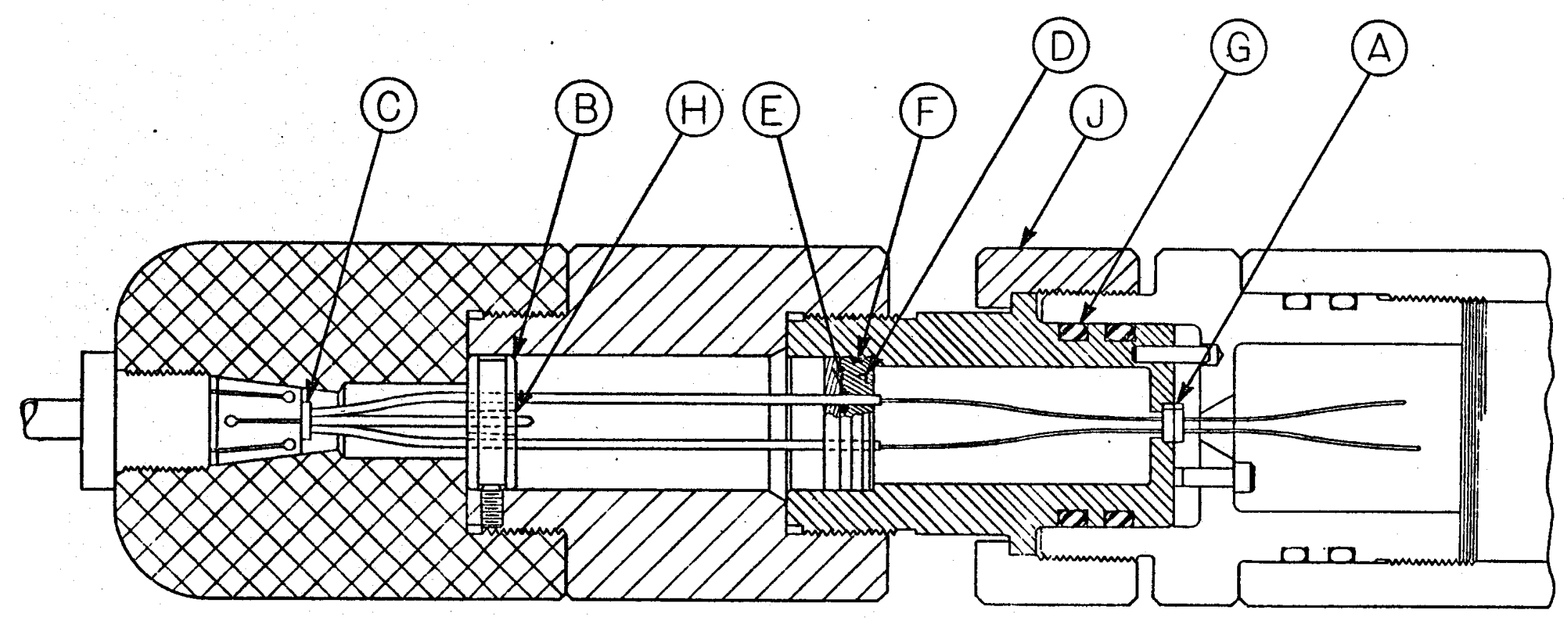

FIGURE 7. SIMPLIFIED ASSEMBLY DRAWING OF OP TICAL CABLE-HEAD. (A) Fiber-optic connector; (B) stress link; (C) armor clamp assembly; (D) pressure barrier; (E, F, G) pressure seals; (H) cable stress member and pull-out clamp; (J) make-up collar. 


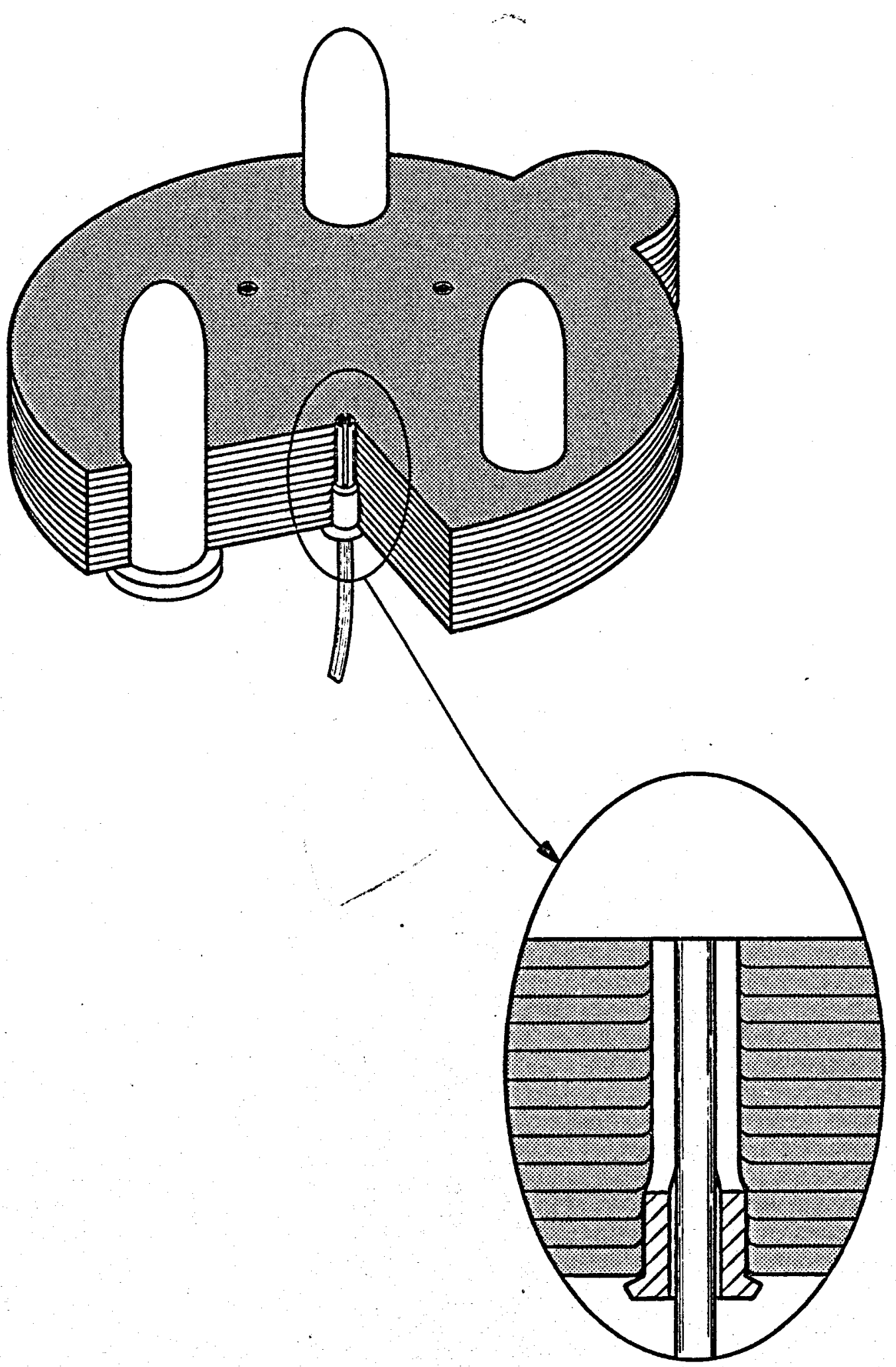

FIGURE 8. SCHEMATIC DRA WING OF OPTICAL CONNECTOR HELD INSIDE THE PRESSURE HOUSING. The detail shows a typical ferrule-fiber assembly held by the photo-etched precision plates. 
these are pushed through one of the alignment disks. The individual holes in this disk are slightly smaller than the OD of the sleeve, causing the slotted sleeve to collapse slightly onto the fiber, holding it in place without adhesive. The three key elements - slotted sleeve, chemically milled disk, and prepared fiberare shown in the photograph in Figure 9.

After the first disk is assembled onto the three fiber assemblies, additional disks are stacked onto succeeding ones, each coated with a thin layer of high temperature adhesive. A total of 11 such disks complete the assembly, which is then clamped until the cement is cured. Then the alignment pins are removed, and the entire front surface, including fiber ends, is optically polished. Both connector halves are prepared in this way, and the precision alignment pins are re-inserted into the male half of the connector.

\section{Adhesives}

A severe problem unique to high-temperature operation concerns the use of adhesives in assemblies containing glass fibers. Optic fiber assemblies in communications applications rely heavily on epoxy-type adhesives to bond the glass to metal, plastic, and ceramic components. Typically, for example, connector assemblies utilize an epoxy to securely fasten individual fibers in alignment ferrules or V-grooves. However, the coefficient of expansion of glass differs seriously from that of the epoxies, and at high temperatures, creates stresses in the glass that both increase losses and eventually result in fracture. An exhaustive study of compounds which might serve as adhesives in this application was conducted during the program. The most favorable solution found is offered by a silicone resin designed as an additive for paints and enamels. The substance is a slightly viscous liquid which air dries at room temperature; it must then be cured at a temperature above the maximum exposure temperature anticipated. In service, it can survive temperatures as high as $350^{\circ} \mathrm{C}$. The cured resin forms a thin, hard but flexible, transparent film which bonds well to most materials. The inherent flexibility of the film allows its use over a wide temperature range. Although it involves a rather lengthy application, dry, and cure cycle, this material has been satisfactorily used to secure fibers in high-temperature connections in this program. The particular resin used is made by Dow Corning, and sold under the name "804 Silicone Resin".

\section{Pressure Seals}

Static pressure seals utilizing O-rings can be successfully employed at temperatures as high as $250-300^{\circ} \mathrm{C}$, utilizing either fluorocarbon rubber or TFE O-rings, if relatively short exposure cycles (of the order of a few hours) are involved. Conventional seals utilizing both materials were investigated, and a pressure housing and connection concept designed. Individual seals were shown at E, F, and $G$ in Figure 7. The critical element in this concept, is the seal which isolates the individual optical fibers (at E). Pressure is isolated from the optical connector by a pressure barrier through which individual fibers must be sealed. The pressure barrier is shown at $D$ in Figure 7 . It consists of a stainless steel piston sealed into the body of the cable head by a conventional O-ring seal. Within this piston are individual seals, called "strangle seals", in which buffered fibers are held in the pressure barrier piston by individual TFE O-rings.

Two factors are critical to the success of this type of seal: first, the pressure must develop sufficient friction between the buffered fiber and the $O$-ring to prevent the fiber from sliding through the pressure barrier; second, the pressure applied to the pressure plate, which creates the sealing force on the O-ring, must not exceed the yield strength of the buffer material. If both these conditions are met, the seal will function. As the temperature is elevated and the yield strength of the buffer material is reduced, the danger of pressure simply extruding the plastic through the hole in the pressure barrier increases. Consequently, a seal of this type that functions satisfactorily at low temperature may not be successful at high temperature. Since both PFA and TFE can be extruded at sufficiently high temperature and pressure, the clearances within the pressure barrier are very critical. 


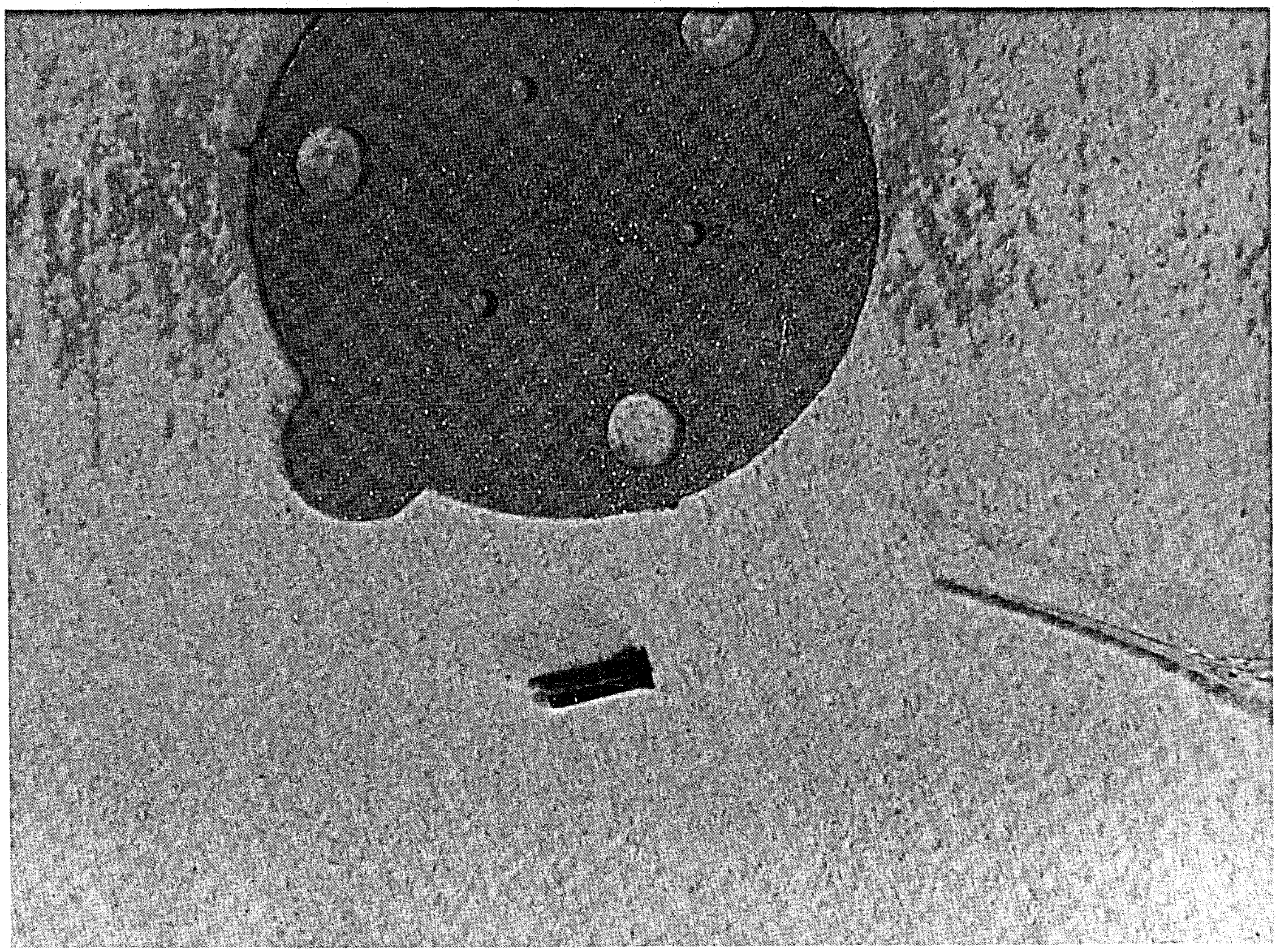

FIGURE 9. PHOTOGRAPH OF A SLOTTED FERRULE, ONE OF THE PHOTO-ETCHED PLATES, AND A STRIPPED 0.068-INCH BUFFERED FIBER 
Static performance of the seal was tested at both room temperature and at elevated temperature in a special test fixture shown, together with the elements of the seal, in Figure 10 . At 7500 psi (maximum pressure used in the test) and room temperature, the seal behaved well. The PFA yielded slightly (an initial creep of approximately 0.040 -in.) and then took a fixed set with no leakage. After the pressure stabilized at 7500 psi, no further creep was observed after two hours. Repressuring cycles produced some hysteresis, but no leakage. This is normal for seals of soft materials on each other, and presents no difficulty as long as allowances are made for the slight movement. After a sufficient number of cycles, it can be anticipated that the seal will fail. Consequently, in service it may be necessary to establish a design life, and then replace the seals prior to that time.

In the laboratory tests, after several pressure cycles to 7500 psi, the test unit was disassembled and the seal examined. In Figure 11 is shown a photomicrograph of the tested seal. Deformation of the TFE O-ring caused by the pressure plate can be noted, together with a slight necking down of the PFA buffering material. This represents satisfactory seal operation at room temperature.

The critical test of the operation of this seal is its performance at high temperature. To test the operating range, a seal was assembled in the test fixture as previously described, and the chamber installed in a high-temperature oven. With the hydraulic system sealed, increasing temperature also resulted in increasing pressure and these two parameters are plotted in Figure 12, which shows the significant results of the test. Pressure gradually increased in the sealed chamber with temperature, to 4000 psi at $269^{\circ} \mathrm{C}$. At that point, a slight drop in pressure was noted. Although no significant leakage was detected, the result can be interpreted to mean that yield of the PFA buffer material had begun to occur. Finally after a total of 2 hours and 10 minutes, the seal failed. The indicated temperature at that point was $276^{\circ} \mathrm{C}$.

The results of this series of experiments reflect the fact that at a given temperature, PFA can be extruded by some appropriate pressure. At $275^{\circ} \mathrm{C}$ the extrusion pressure is about 4000 psi and the seal will fail. The critical temperature for this particular seal under the test conditions can be said to be $265^{\circ}$ to $270^{\circ} \mathrm{C}$.

Tests of the outer pressure barrier seal at elevated temperature and pressure were conducted without incident. An identical but undrilled pressure piston was tested with both TFE and Viton (fluorocarbon) O-rings. Both operated satisfactorily with no leakage at pressures of 12,000 psi and temperatures of $275^{\circ} \mathrm{C}$ for 2 hours.

\section{Status of Cable Head Design}

During the program, individual components of the cable head have been constructed and tested, but a complete assembly has not been produced. Delays and uncertainties in the cable procurement made it impossible to fully evaluate the operation of this assembly. Based on tests of the coupling efficiency of the optical connector, it is anticipated that optical losses can be held to approximately $4 \mathrm{~dB}$ in each fiber connection, over the temperature range of the cable and cable seals.

\section{OPTICAL SENSOR DEVELOPMENT}

The principal advantage of a fiber optic approach over the use of electrical cable, is that the problems associated with the transmission of electrical power and signals at high temperatures can be avoided. However, to fully realize this advantage the system must be totally optical, that is, sensors are required that operate without electrical power. To perform a well-logging function, such a sensor must possess an optical property that is influenced by the bore hole parameter of interest in such a way as to modulate, or to emit, optical radiation. Because the optical signal produced by the sensor is to be carried over a long distance by an optical fiber, it is also essential that the optical modulation or emission take place in the spectral band where fiber optic attenuation is minimized, namely, from about $800-n m$ to 


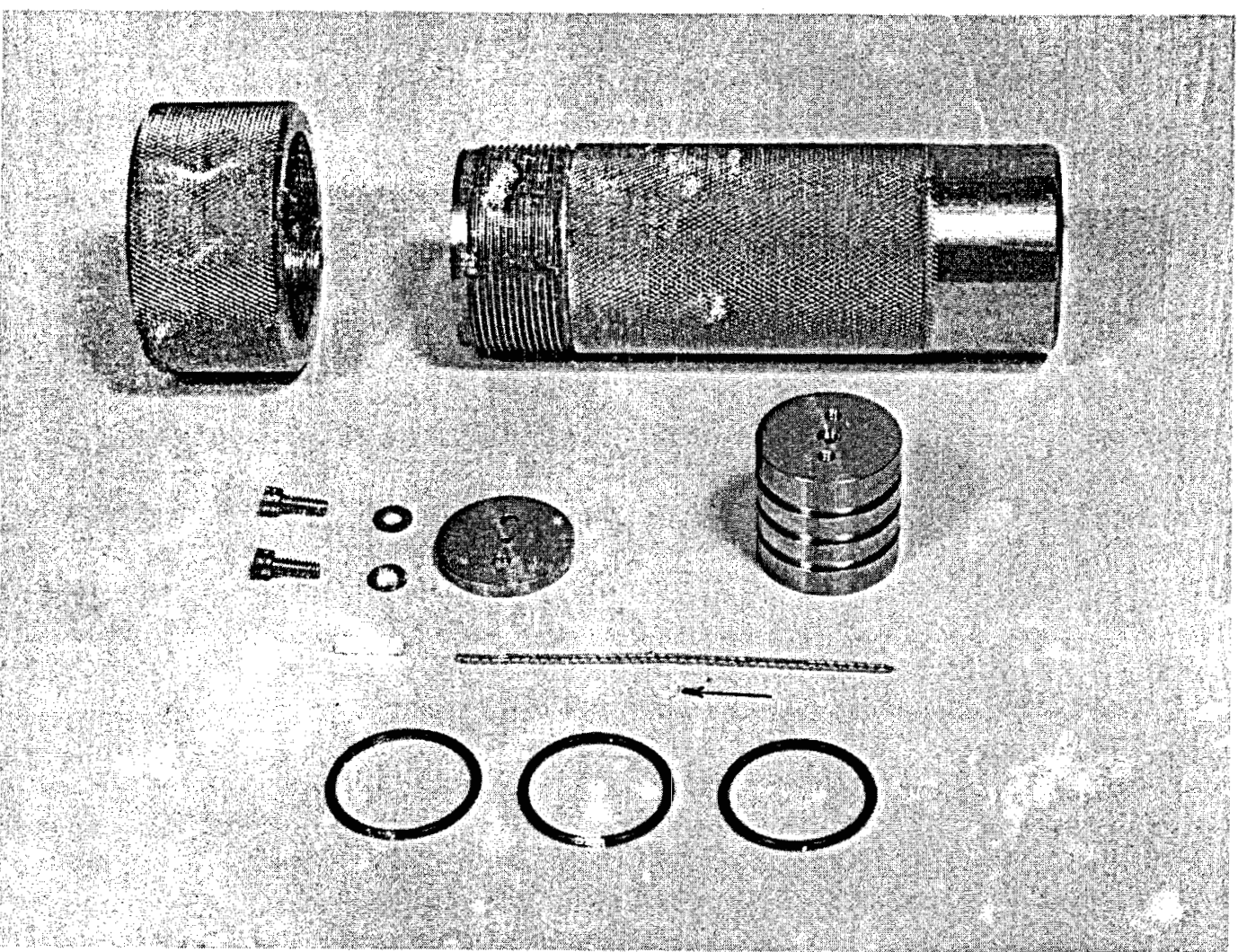

(a)

$\stackrel{m}{\stackrel{m}{*}}$

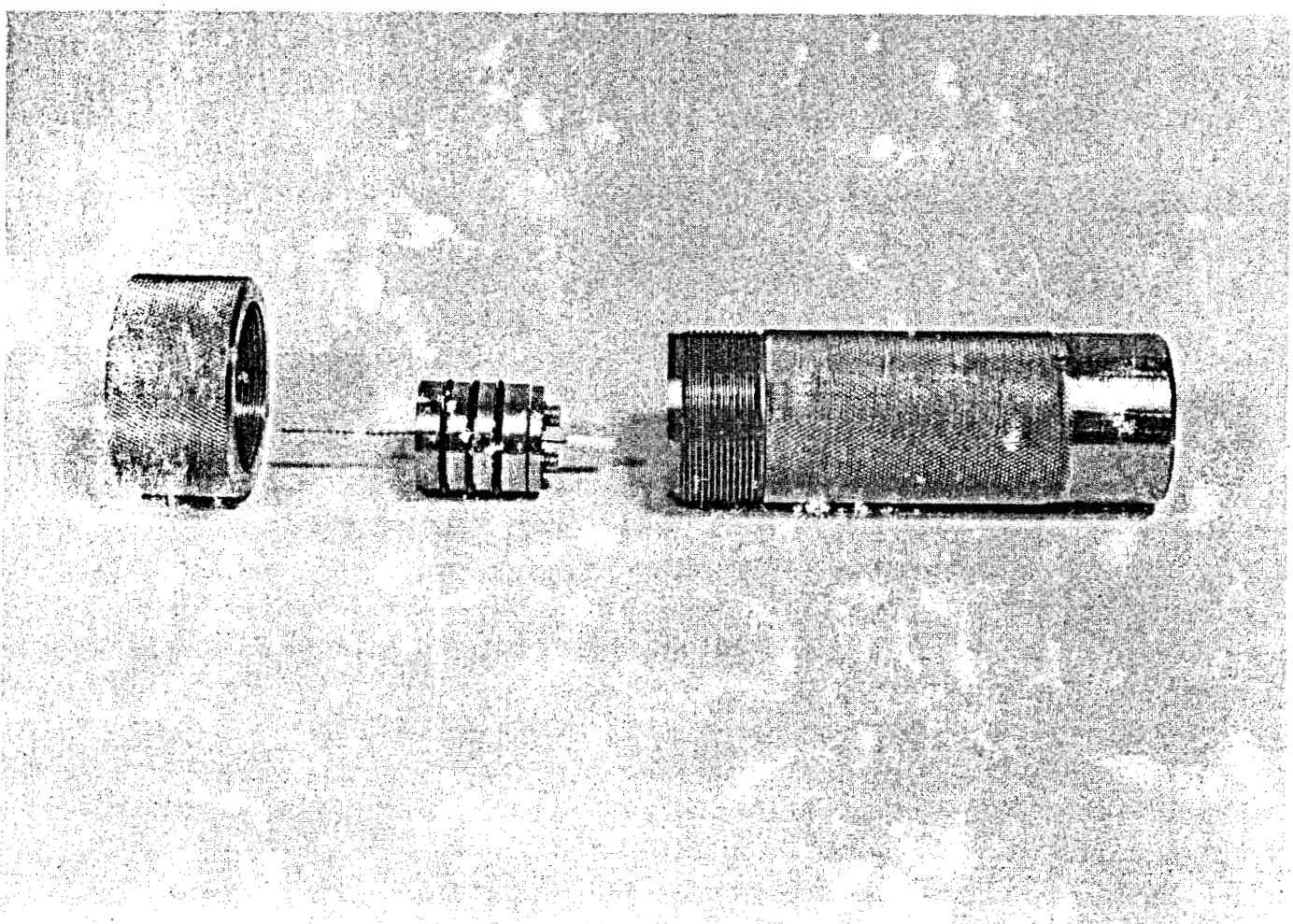

(b)

FIGURE 10. MINIATURE PRESSURE CHAMBER AND PRESSURE-BARRIER TEST FIXTURE. (a) seal components; (b) as sembled pressure barrier ready for test. The arrow in upper photo points to miniature TFE O-ring. 23 
$\stackrel{\stackrel{H}{N}}{\stackrel{+}{*}}$

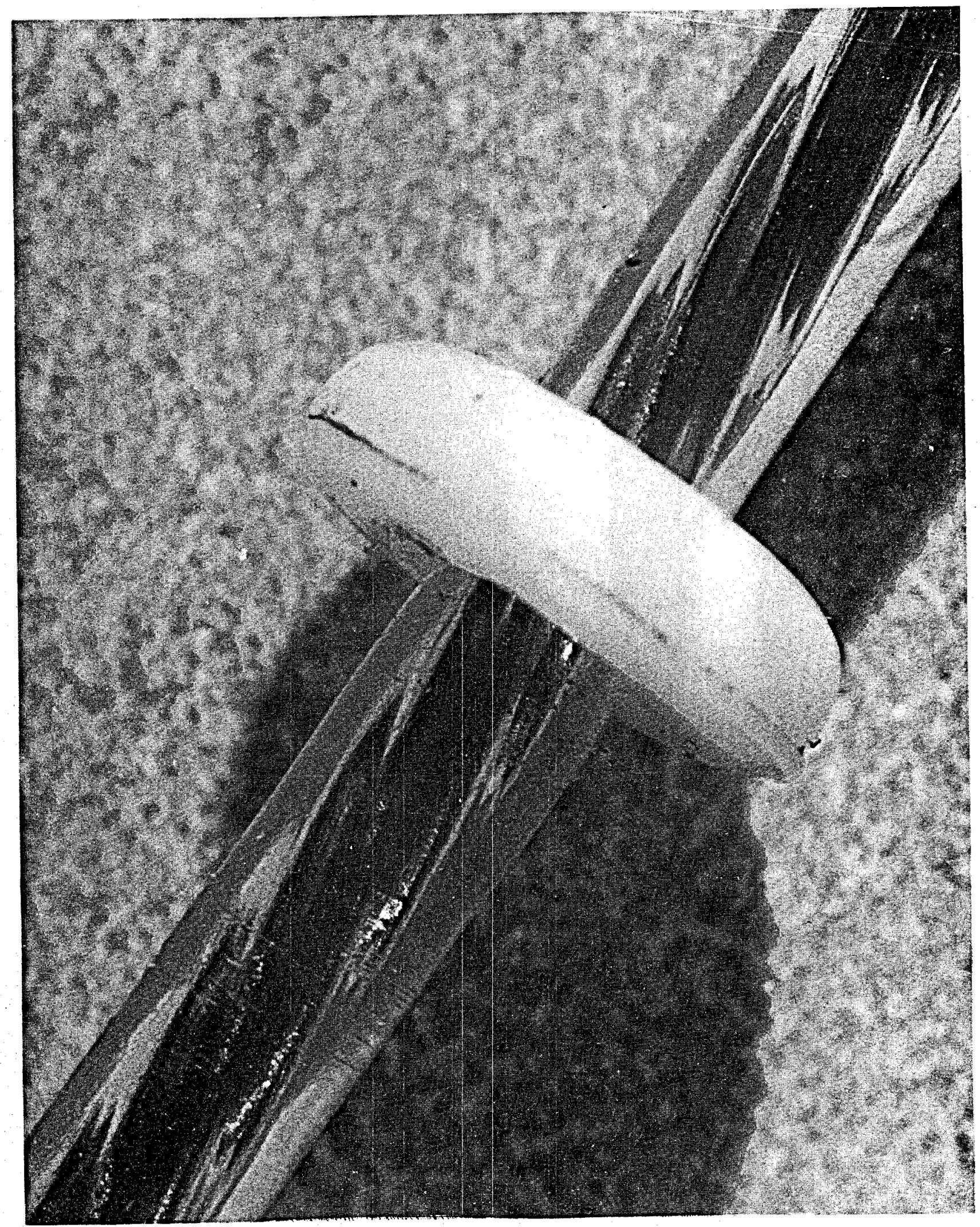

FIGURE 11. PHOTOMICROGRAPH (45X) of TFE-on-PFA PRESSURE SEAL AFTER PRESSURE TEST. 7500 psi at $25^{\circ} \mathrm{C}$ for two hours. 
FIG 12: PRESSURE-TEMP PLOT FOR TFE O-RIHG SEAL OH PFA BUFFERED OPTICAL FIBER

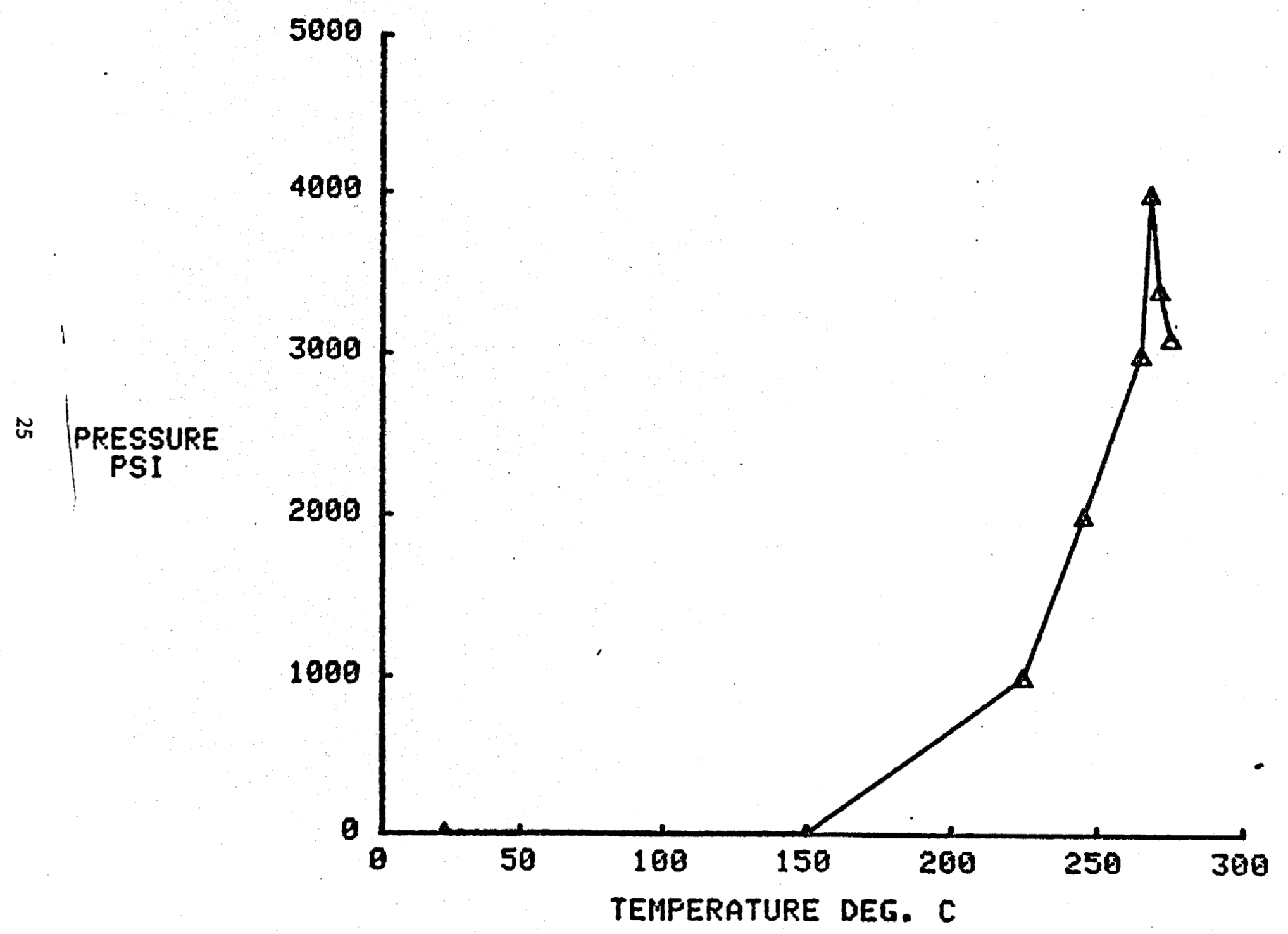


$1100-\mathrm{nm}$. Finally, it is also desirable, but perhaps not essential, that the method of measurement be insensitive to signal intensity fluctuations caused by variable stresses in the fiber itself.

The objectives of the sensor development phase of this program were to identify and assess the feasibility of sensor concepts that satisfy these requirements. In the discussion that follows are reported the findings on promising approaches to the measurement of temperature, neutron flux and pressure.

\section{A. Temperature Logging}

Temperature logging is recognized by most authorities ${ }^{(5)}$ as the highest priority requirement of geothermal bore hole measurement. Aside from the obvious interest in reservoir fluid temperature, continuous temperature logs can provide important information on formation lithology, bed boundaries, thief zones, and well conditions generally. Temperature surveys in conjunction with flow tests are also significant in predicting the thermal performance of a reservoir in production. Eventually, properly conducted temperature tests may prove as basic to geothermal reservoir analysis, as resistivity measurements have been to petroleum reservoir assessment. Consequently, development of an optical temperature sensor capable of application to continuous well logging, was a fundamental objective of this program.

In view of the general requirements of optical well-logging sensors, the search for an approach to temperature logging by optical means began with a review of the temperature dependent optical properties of solids. As a result of this review, two general measurement approaches were tentatively identified as promising. These were the measurement of color center annealing rates and optical absorption spectroscopy. Parallel studies of both approaches were undertaken to select a single concept for further development. This work is described below in Section A.1.

The next tasks, which are discussed in following paragraphs, involved the selection of a sensor material and analyses of temperature measurement methods. Once this was complete, an extensive series of experiments was performed, designed to answer certain fundamental questions and to test the overall sensor concepts. The design and preliminary testing of a prototype, instrument is reported in Section A.7. Other details of the temperature sensor investigation appear in Appendix A and Appendix B.

\section{Preliminary Studies}

a. Color Center Annealing. One approach to the optical measurement of temperature involves the determination of color center annealing rates. The transducer in this case is a material that is normally transparent in the near infrared but which, for present purposes, is made to absorb infrared radiation at certain wavelengths through the introduction of "color centers". In this concept, the material, in the form of a small rod or crystal, would be coupled at each end to a separate optical fiber in the optical cable. An optical carrier beam (at the infrared wavelength) would be transmitted from a source at the surface down one fiber, through the transducer, and back to a receiver at the surface through the other fiber.

If the wavelength of the incident radiation lies within the absorption band of the color centers, as long as a significant number of centers remain in the sensing element, the intensity of the signal at the receiver will be attenuated by absorption in the sensor. For color centers with the desired thermal properties, this will be the case at temperatures lower than those of interest.

However, when the sensor is raised to a sufficiently high temperature, thermal annealing, which causes destruction of the color centers, will proceed at a rate that depends on temperature. As a result, the signal intensity at the receiver will increase continuously at the same rate. Thus, once the temperature dependence of the annealing rate has been determined, a measurement of the slope of the signal vs. time curve will determine the temperature of the environment in which the sensor is located at a particular instant. 
As a specific example of how this idea might be implemented, consider the characteristics of the so-called " $M$ " center in potassium chloride (K $C l)$. This center, which is thought to consist of a $K^{+}$ion, two negative ion vacancies, two positive ion vacancies and a trapped electron, gives rise to an absorption band centered at about $820 \mathrm{~nm} .^{(6)}$ This wavelength lies near a minimum in the fiber absorption curve and is, therefore, nearly ideal for the intended application.

The thermal annealing characteristics of the $M$ center in $K C$ lare also favorable, at least for the measurement of temperatures up to about $300^{\circ} \mathrm{C}$. This is illustrated in Figure 13 where are shown calculated rates of decrease in optical absorption at $820 \mathrm{~nm}$ as a function of time and temperature. These calculations are based on Tomiki's $(7)$ observation that the decay of absorption due to $M$ center annealing follows a simple exponential law. Thus, if $D(t)$ is the optical density of a specimen at time $t$ and $D(0)$ is the density at $t=0$ (when annealing is initiated), we have

$$
D(t) / D(0)=e^{-t / \tau}
$$

where, according to Tomiki's experimental data,

$$
1 / \tau=2.01 \times 10^{12} \mathrm{e}^{-1.31 / k T}
$$

with $\tau$ in seconds and $k T$ in electron volts ( $k$ is the Boltzmann constant and $T$ is the temperature in ${ }^{\circ} \mathrm{K}$ ).

The two important points illustrated here are the following: (1) The annealing rate is quite sensitive to temperature and can therefore provide an accurate measure of temperature in the range indicated; (2) for a limited range of temperatures the times required for an accurate measurement of the annealing rate are convenient. Thus, at the lowest temperature indicated here $\left(200^{\circ} \mathrm{C}\right)$ one would accumulate optical transmission data for perhaps 20 seconds to determine the relaxation time (which, according to Eq. (2), determines the temperature), while at $275^{\circ} \mathrm{C}$ a measurement interval of a few seconds is required. The method may also be used at lower temperatures, perhaps as low as $150^{\circ} \mathrm{C}$, although data accumulation times of severai minutes may then be required. However, at temperatures much above $300^{\circ} \mathrm{C}$ the $\mathrm{M}$ center annealing rate is so rapid that virtually all $\mathrm{M}$ centers are destroyed in times that are impractically short for well logging purposes.

In addition to being limited to a rather narrow range of temperatures, the $M$ center annealing approach suffers from the fact that once the color center population is depleted, the crystal is useless as a temperature sensor. One might reactivate the sensor by the in-situ production of color centers through gamma irradiation. However, because a very intense gamma ray source is needed for this purpose, in situ reactivation is probably impractical. Our study of the $M$ center annealing approach was therefore terminated with the conclusion that its usefulness is too limited for well-logging purposes.

In our continued search of the literature on high-temperature color center annealing we encountered an experimental study of centers in $\mathrm{KBr}$ that seemed promising. ${ }^{(8)}$ The particular center of interest in this case is actually a very small potassium particle that precipitates at high temperatures in very pure $\mathrm{KBr}$ crystals that contain an excess concentration of $\mathrm{K}^{+}$ions. The properties of these particles that are of interest in the present context are the following:

- The onset of precipitation occurs at about $150^{\circ} \mathrm{C}$ and is accompanied by a decrease in $\mathrm{F}$ center density;

- The particle density reaches a maximum at about $260^{\circ} \mathrm{C}$;

- As the temperature is raised above $260^{\circ} \mathrm{C}$ the potassium particles begin to decay giving rise to an increase in the density of $F$ centers; 


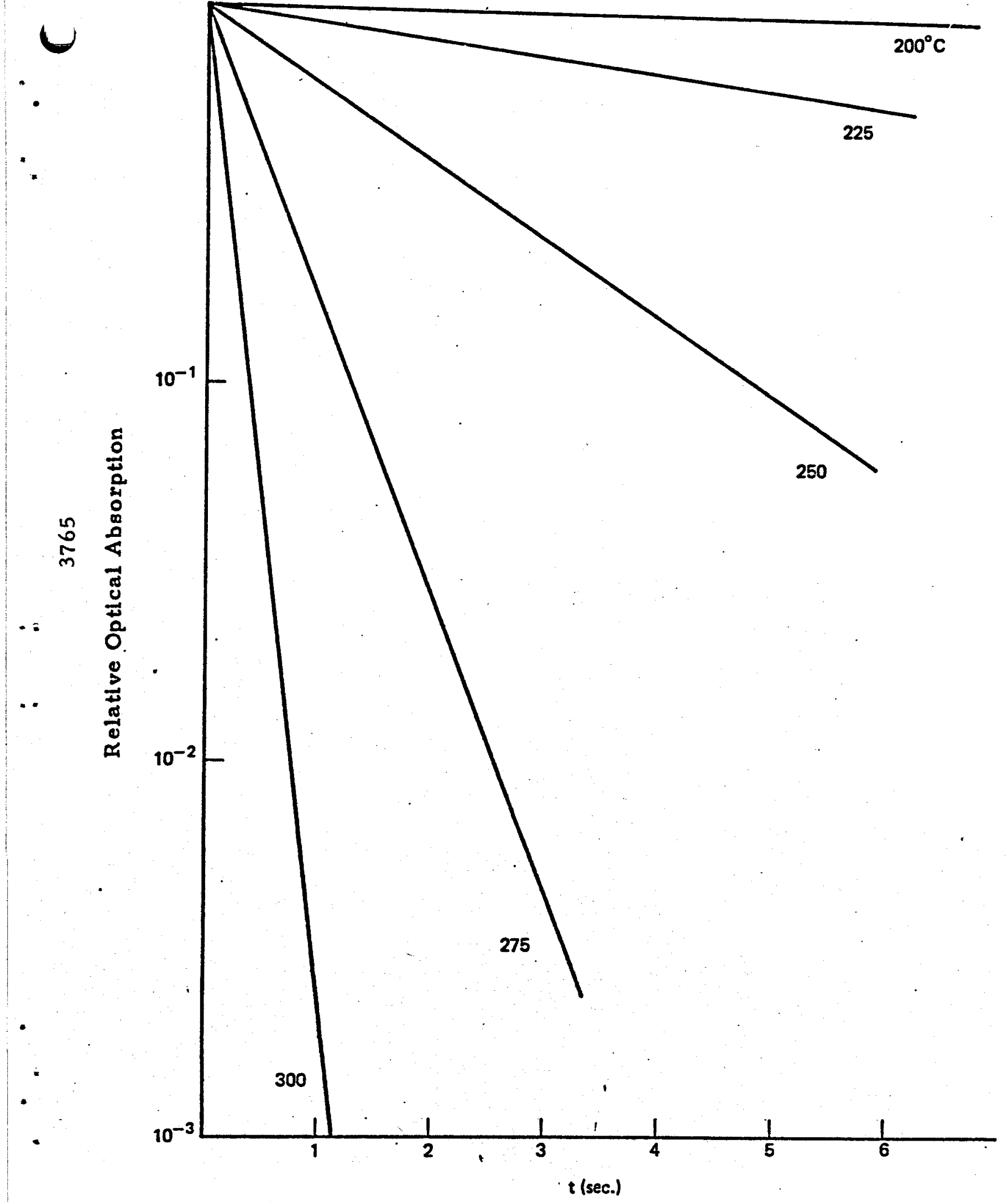

FIGURE 13. DECR EASE IN OPTICAL ABSORPTION CAUSED BY ANNEALING OF M CENTERS IN KCL 
- Optical absorption by the equilibrium concentration of particles is measurable at temperatures up to about $350^{\circ} \mathrm{C}$;

- The optical absorption spectrum peaks at about $790 \mathrm{~nm}$ in very pure $\mathrm{KBr}$ and at somewhat shorter wavelengths when impurities are present;

- From the theory of the formation of such particles in alkali halide crystals ${ }^{(9)}$, it would appear that the precipitation process is reversible, i.e., after complete annealing at temperatures above $350^{\circ} \mathrm{C}$, particles are reformed by cooling.

These facts suggested that it may be possible to use measurements of optical absorption by $\mathrm{KBr}$ at $790 \mathrm{~nm}$ (which is an appropriate wavelength for signal propagation in optical fibers) to infer temperatures in the $150^{\circ} \mathrm{C}$ to $350^{\circ} \mathrm{C}$ range. This might be done by monitoring equilibrium particle densities, or, given more information on the particle growth and decay rates, by measuring such rates in much the same way as with the $M$ center in $\mathrm{KC}$.

However, further research led us to conclude that it is not practical to monitor equilibrium densities because the approach to equilibrium is too slow, typical times to approach equilibrium being of the order of 30 to 60 minutes. ${ }^{(10)}$ The analysis of particle growth or decay rates, on the other hand, continued to show promise, mostly because experimental studies of such processes revealed that a change in temperature causes a rather rapid transient in particle density for a short time after the change. A study of the kinetics of these initial transients was then undertaken, following the theoretical model proposed by Calleja and Agullo-Lopez. (11) We soon concluded that, while it is feasible to measure growth and decay rates, the interpretation of these data in terms of the temperature of the specimen is not straightforward because the growth and decay rates depend, in a complicated way, on the particle size distribution at the time the specimen temperature is changed. This fact, together with continued progress in the parallel study of absorption spectroscopy methods, led us to abandon the color center approach in favor of a spectroscopic method.

b. Absorption Spectroscopy. The general sensor concept in this case is similar to that described above in that one would employ a source and detector outside the hole, connected to a down-hole passive transducer by a fiber optic cable.

However, with the spectroscopic approach, the objective is to sense some temperature dependent feature in the absorption spectrum of the transducer material. One therefore requires a broadband source of near infrared radiation and a wavelength analyzer, such as a monochromator, to determine the spectral content of the radiation transmitted by the sensor.

The restriction to wavelengths in the $800-1000 \mathrm{~nm}$ band means that the spectral features of concern must correspond to electronic transitions because absorption by the crystalline lattice occurs only at much longer wavelengths. There are, therefore, two types of transitions of interest - those corresponding to defect absorption and those corresponding to some fundamental absorption property of the host crystal. Defect absorption bands are generally rather broad and featureless, particularly at high temperatures, and, except for thermal broadening, do not exhibit any easily detected change with temperature. There is, on the other hand, at least one absorption feature of some crystalline solids, namely, the position of the so-called fundamental absorption edge in insulators and semiconductors, that does exhibit an easily detected shift as the temperature is changed. Thus, very early in the program, our study of the spectroscopic approach was narrowed to absorption edge spectroscopy.

Experimentally, the fundamental absorption edge is observed as a sharp increase in the transparency of a crystal as the wavelength is increased past some critical value. This is illustrated in Figure 14, which shows the spectrum transmitted by a thin wafer of gallium arsenide (GaAs) at various 


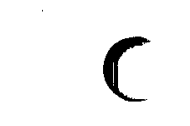

-

4443

TEMP =

$200,250,300,350,400$ CENTIGRADE

$\ddot{\sigma}$

HORMAL TZED

RELATIUE

INTENSITY

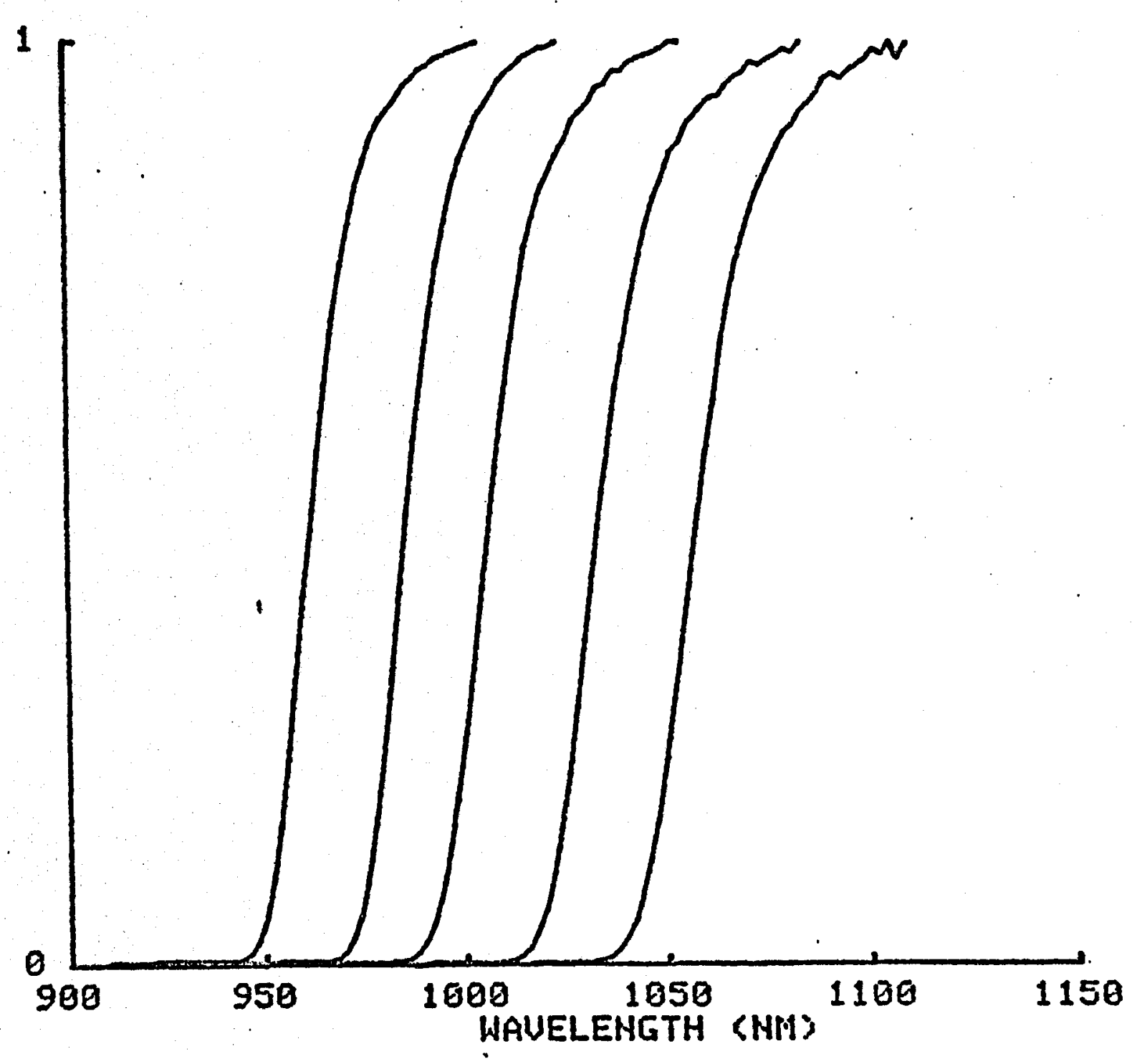

FIGURE 14. EXPERIMENTALLY MEASURED OPTICAL TRANSMISSION OF GaAs vs. WAVELENGTH AND TEMPERA TURE. 
temperatures. These data were obtained experimentally by a method to be described later. For the present, the important point is that the absorption edge spectrum, which is that part the of curve where the transmission increases rapidly with increasing wavelength, shifts markedly to longer wavelengths at higher temperatures. This shift of the absorption edge with temperature is the basic phenomenon on which the temperature sensor concept; which was eventually developed, was founded.

\section{Selection of Sensor Material - GaAs}

The physical nature of absorption edge transitions together with the restriction to the $800-1100 \mathrm{~nm}$ band, limits the class of suitable materials to wide band gap semiconductors. This is because the absorption edge corresponds to photon induced electronic excitation across the energy gap. Thus, if the photon energy is greater than the band gap energy, such transitions can occur and the probability of photon absorption is large. If, on the other hand, the photon energy is too small to cause band gap transitions, the absorption probability will be small. The absorption edge spectrum therefore occurs in a band centered near the band gap energy. It follows that if we must work with spectra in the $800-1100 \mathrm{~nm}$ band, which corresponds to photon energies from about $1.1 \mathrm{ev}$ to $1.5 \mathrm{ev}$, the only materials we need consider are semiconductors with band gap energies in this range.

Another important consideration is the rate at which the absorption edge shifts with increasing temperature, or, equivalently, the rate of change of the band gap energy with respect to temperature. One would like to have this parameter as large as possible so that a small change in temperature would produce a measurable shift in the location of the edge. Fortunately, this parameter has been measured for all materials of interest.

Other criteria include the steepness of the edge, and thermal properties such as melting point, coefficient of thermal expansion and specific heat. However, because the values of these parameters are nearly the same for materials with similar band gap energies (with one exception to be noted later), consideration of these properties did not enter the selection of a sensor material.

Finally, it is evidently quite essential that the material selected be available, at reasonable cost, in a form suitable for absorption edge measurements. This requires very thin, highly polished wafers of the sensor material.

Data on some of the more important parameters are listed in Table I for several semiconductors with energy gaps in the appropriate range. From these data it can be seen that neither of the elemental semiconductors, silicon and selenium, are suitable because the band gap for silicon is too small at $200^{\circ} \mathrm{C}$ while selenium melts at too low a temperature. On the other hand, all of the compound semiconductors listed here are potentially useful and, in fact, have similar properties as far as the parameters of interest are concerned. Our first choice for a sensor material, GaAs, was therefore based primarily on its low cost availability in a suitable form. Indium phosphide was chosen as a backup material because its absorption edge lies near the minimum in the fiber optic absorption spectrum at temperatures between $200^{\circ} \mathrm{C}$ and $300^{\circ} \mathrm{C}$.

\section{3. "Maximum Slope" Detection}

Although gallium arsenide has one of the steepest absorption edges of all wide band gap semiconductors, the edge spectrum still covers several nanometers, as can be seen from figure 15. Thus, unless one can identify and accurately locate some feature of the spectrum at a particular wavelength, the width of the absorption edge leads to an uncertainty in its location and this, in turn, leads to an uncertainty in the temperature measurement. One could improve the situation to some extent by choosing, as a reference point, the wavelength at which the transmitted intensity reaches some arbitrary value. However, this approach, which is used in the laboratory to measure the temperature dependence of the band gap energy, requires careful control of the source intensity and losses in the measuring system. As 
TABLE I. ENERGY GAPS, THEIR TEMPERATURE DERIVATIVES, AND MELTING POINTS OF SELECTED SEMICONDUCTORS (REF. 6)

\begin{tabular}{lcccc} 
& $\begin{array}{c}\mathrm{Eg} \\
\left(\mathrm{ev} \text { at } 20^{\circ} \mathrm{C}\right)\end{array}$ & $\frac{\mathrm{Eg}}{\left(\mathrm{ev} \text { at } 200^{\circ} \mathrm{C}\right)}$ & $\frac{c}{\mathrm{dEg} / \mathrm{dT}}$ & \multicolumn{2}{c}{$\begin{array}{c}\text { Melting Point } \\
\left({ }^{\circ} \mathrm{C}\right)\end{array}$} \\
$\mathrm{Si}$ & 1.11 & 1.07 & -14.0 & 1412 \\
$\mathrm{Se}$ & 1.74 & 1.49 & -4.0 & 1060 \\
$\mathrm{AlSb}$ & 1.6 & 1.53 & -5.0 & 1280 \\
$\mathrm{GaAs}$ & 1.43 & 1.34 & -4.6 & 1055 \\
$\mathrm{InP}$ & 1.28 & 1.20 & -4.6 & $>1258$ \\
$\mathrm{CdSe}$ & 1.74 & 1.66 & -4.1 & 1098 \\
$\mathrm{CdTe}$ & 1.50 & 1.43 & & -
\end{tabular}

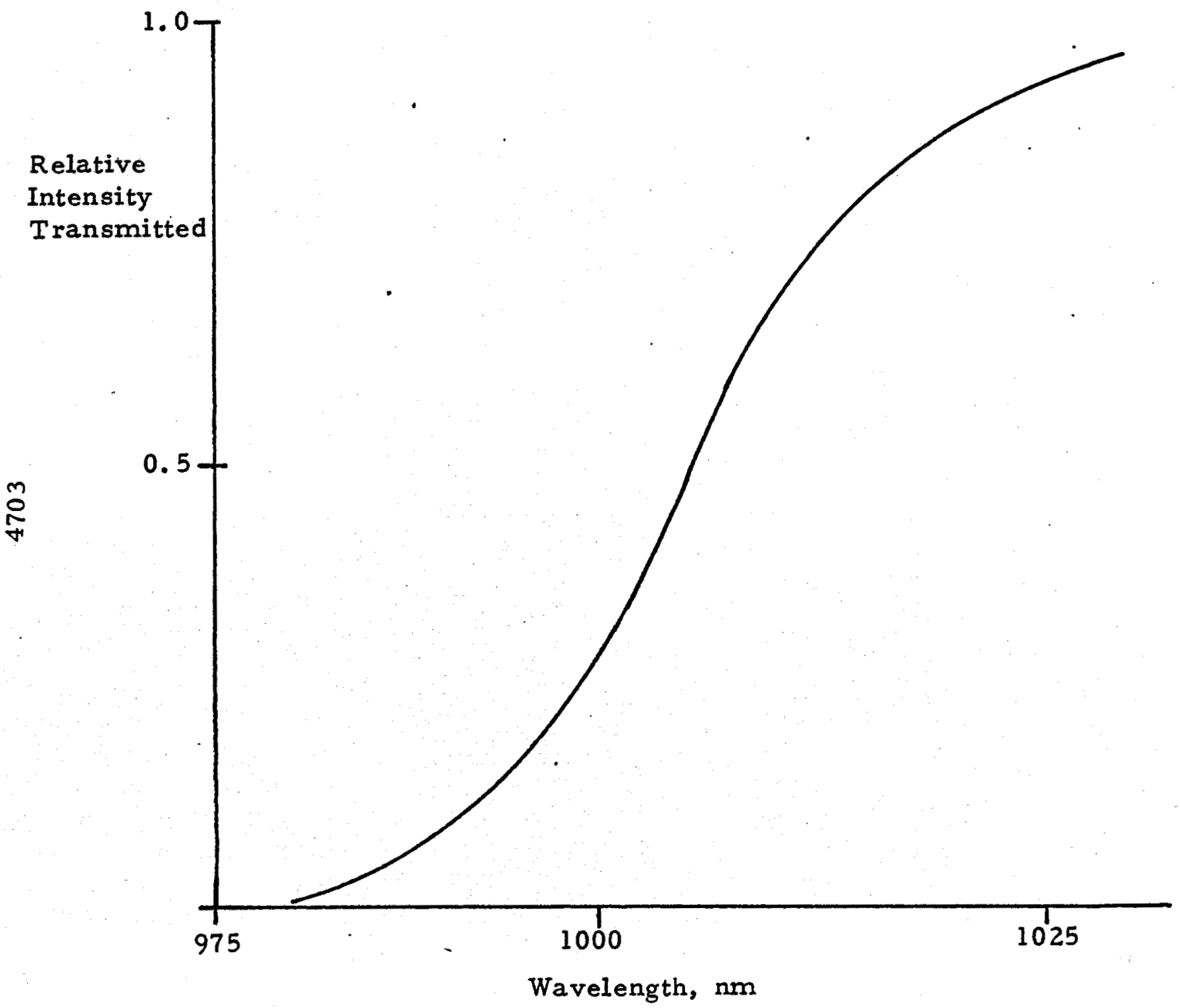

FIGURE 15. GaAs ABSORPTION EDGE AT $300^{\circ} \mathrm{C}$, EXPANDED SCALE 
noted earlier, such control would prove extremely difficult in a well-logging system because losses in the fiber optic cable are stress dependent. Thus, the first problem faced in deciding how to use the absorption edge method was that of finding some feature in the spectrum that can be used as a reference point.

One approach is suggested by a method sometimes used to measure the band gap energy itself.(12) To illustrate, Figure 16 shows the absorption coefficient (the probability per unit length that a photon will be absorbed) for $\mathrm{GaAs}$ at room temperature. This coefficient is easily derived from an experimental transmission spectrum by the method outlined in Appendix A. The data shown here, however, were calculated from a semi-empirical relationship known as Urbach's rule, which accurately describes the energy dependence of the absorption coefficient for many semiconductors and insulators. From the mathematical form of Urbach's rule, which is also discussed in Appendix A, it can be shown that the derivative of the absorption coefficient with respect to energy exhibits a sharp peak at the band gap energy, as shown in Figure 17. Thus, one may use the point of maximum slope in the absorption coefficient as a temperature dependent reference point in the absorption edge spectrum. As shown in Appen$\operatorname{dix} A$, the data processing steps required to find this point are rather simple and are easily handled by a small computer.

There is, however, a practical difficulty in obtaining the required spectrum. As can be seen from Figures 16 and 17, determination of the point of maximum slope requires that accurate data be obtained at photon energies near $1.43 \mathrm{ev}$ (at room temperature) where the absorption coefficient has a value of several thousand reciprocal centimeters. Because the absorption coefficient is so large at this point, one must work with extremely thin crystals to obtain transmitted intensities large enough for accurate measurement. This is illustrated in Figure 18 where the calculated percent transmission at the band gap energy is shown as a function of specimen thickness. From this curve it can be seen that in order to obtain even $10 \%$ transmission, GaAs wafers less than 0.2 mils ( -5 microns) thick must be used. While it may be feasible to produce such a specimen (by polishing a thin crystal glued to a transparent substrate, for example) it presents formidable difficulties at this stage. Thus, a method that involves measurements at smaller values of the absorption coefficient, and can therefore be applied to thicker crystals, is preférable.

From a search of the literature on optical absorption in GaAs, at least one published indication of an Urbach-type behavior in the weak absorption tail of the edge spectrum at high temperature was found.(73) These data, which are presented in Figure 19, show a definite "knee" in the weak absorption tail, and thus indicate that there is a point of maximum slope at a small value of the absorption coefficient. This suggests that it should be possible to apply the maximum slope method to thicker crystals by working with the weak absorption tail rather than that part of the spectrum near the band gap.

There are, on the other hand, still other experimental studies reported in the literature in which the observed absorption spectrum does not contain a point of maximum slope in the weak absorption part of the spectrum. ${ }^{(14,15,16)}$ In these studies the addition of impurities produced only a shift in the position and slope of the edge, thus suggesting that the effect noted above is not caused by impurities alone. All such experiments were, however, performed at or below room temperature. This may mean that the additional knee observed at high temperature is caused, in part, by free carrier or optical phonon absorption, both of which become more prevalent as the temperature is increased. ${ }^{(17)}$ Still, at the time this study was undertaken, there was no conclusive evidence to this effect.

The situation, therefore, was one of some uncertainty. There was, on one hand, published experimental data that indicated that we could apply the maximum slope method to the weak absorption tail, and could therefore use relatively thick crystals in our temperature sensor. On the other hand, there were also indications that the maximum slope method will work only at band gap wavelengths, which would require the use of extremely thin crystals. Because of this uncertainty we undertook an experimental study intended to first determine the usefulness of the maximum slope method with thicker crystals, and 


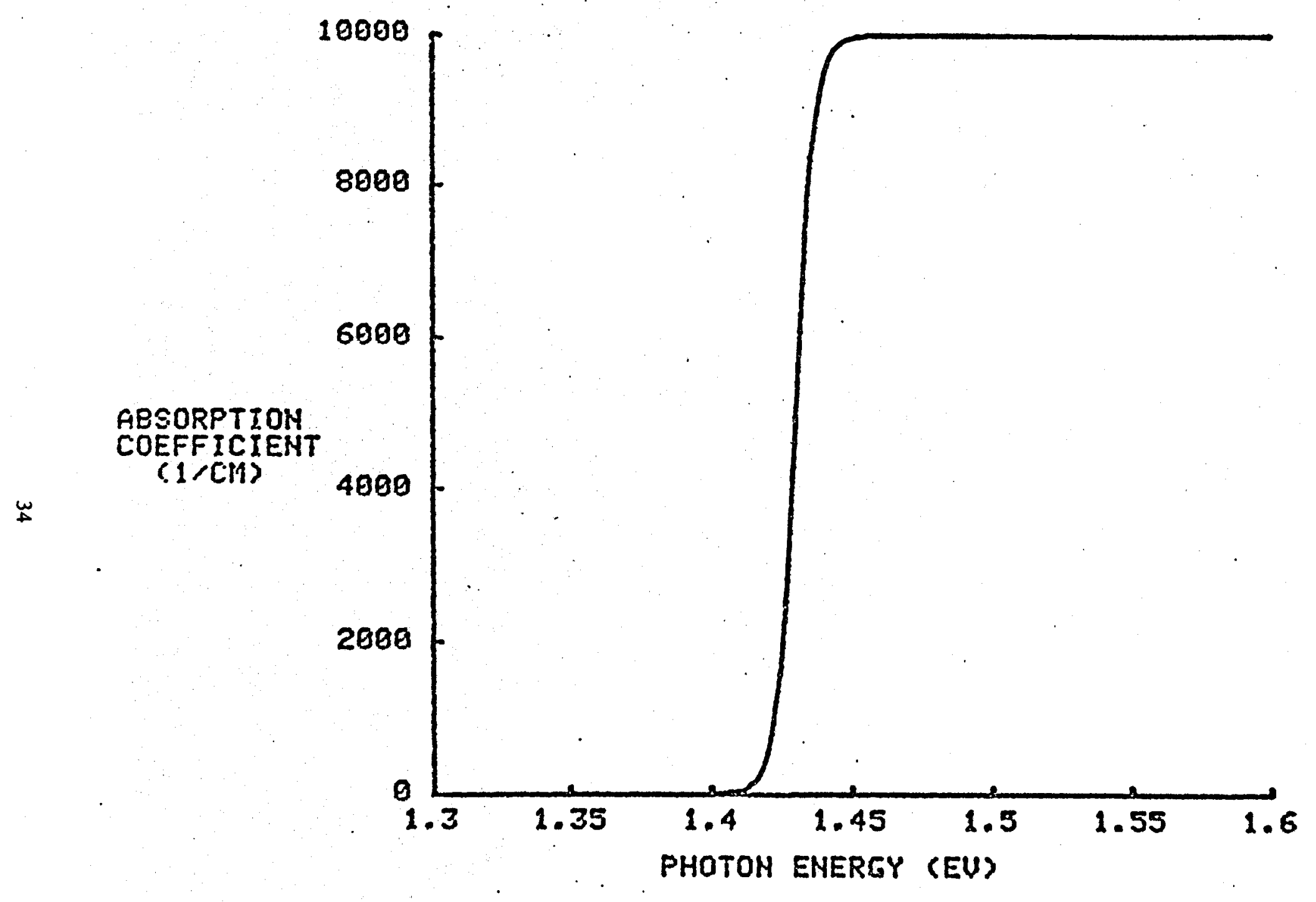

FIGURE 16. PLOT OF THE THEORETICAL ABSORPTION EDGE IN GALLIUM ARSENIDE AT ROOM TEMPERATURE 


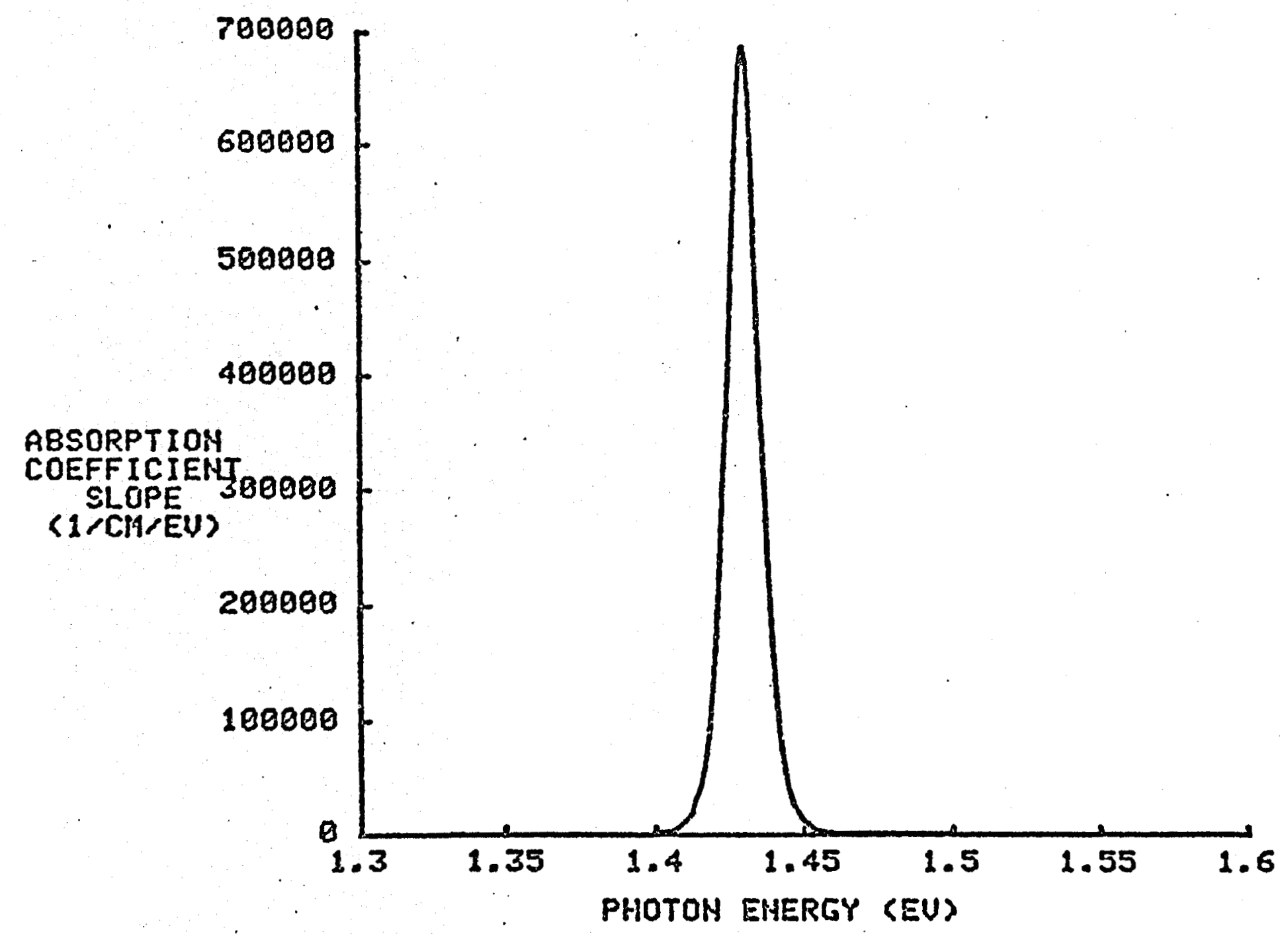

FIGURE 17. PLOT OF THE SLOPE OF THEORETICAL ABSORPTION SPEC TRUM IN GALLIUM ARSENIDE AT ROOM TEMPERATURE 


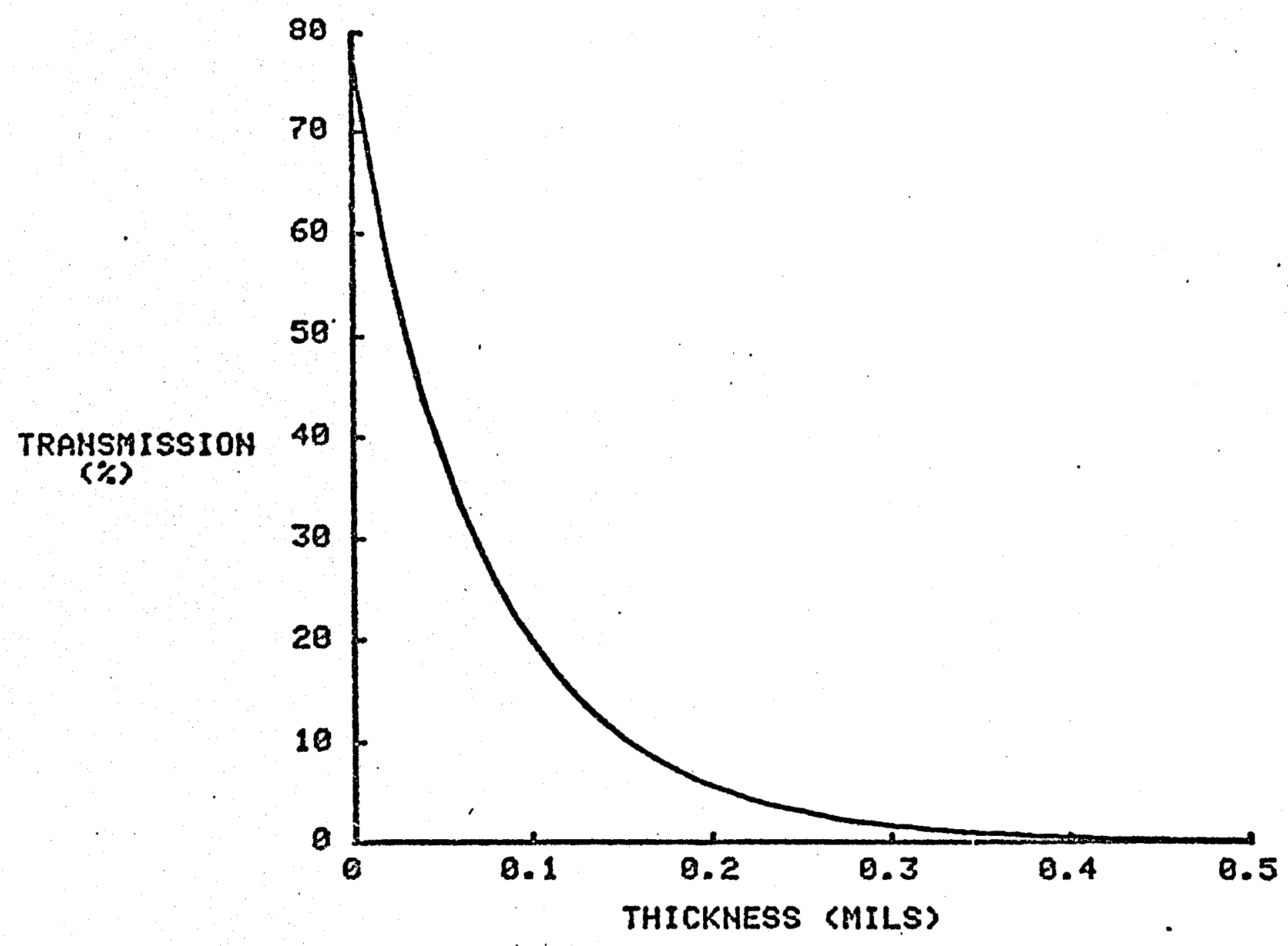

FIGURE 18. PLOT OF LIGHT TRANSMITTED VERSUS SAMPLE THICKNESS AT THE MAXIMUM SLOPE POINT OF THE ABSORPTION COEFFICIENT 

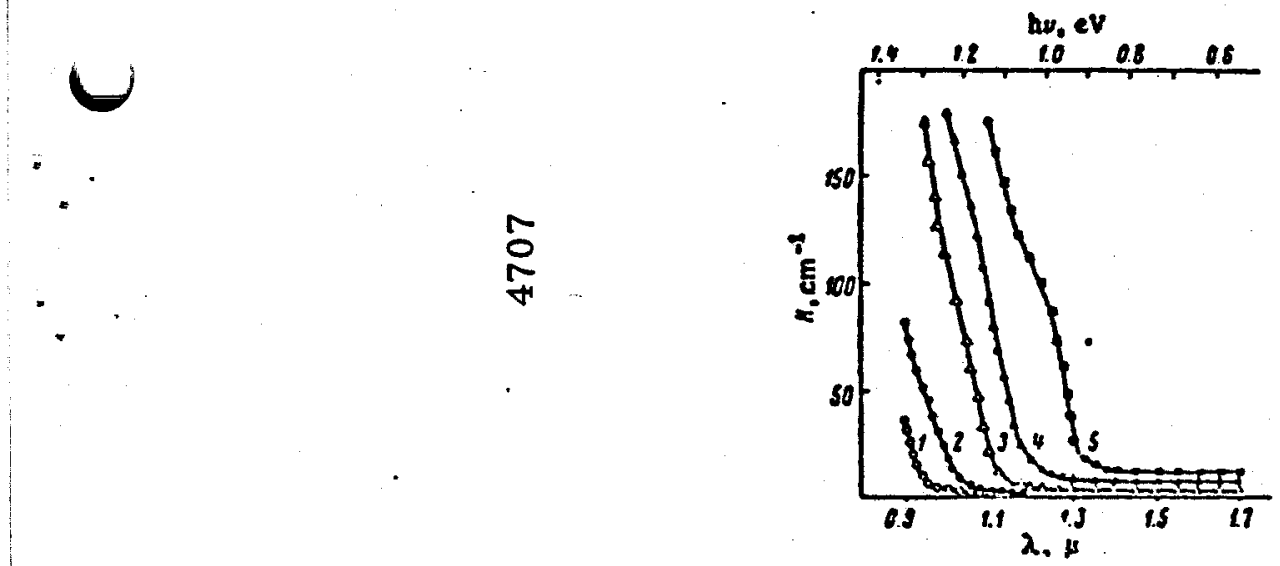

FIGURE 19. ABSORPTION SPECTRA OF N-TYPE GaAs (N=7 $\left.\times 10^{17} \mathrm{~cm}^{-3}\right)$ OBTAINED AT VARIOUS TEMPERATURES T $\left.\left(0^{\circ} \mathrm{K}\right)=1\right) 300$;

2) 473 ; 3) 673 ; 4) 873 ; 5) 1073 (FROM REF. 13)

then, if necessary, to investigate the preparation and use of thin specimens in fundamental absorption edge measurements. The principal results of these experiments are described below; a more detailed account is given in Appendix $A$.

\section{Experimental Investigation}

The basic experimental apparatus used to evaluate the absorption edge temperature approach is shown schematically in Figure $\mathbf{2 0}$ and in the photograph in Figure 21. It consists of a gallium arsenide disk, with optically polished faces, placed in a specially designed metal holder, mounted accurately in the high-temperature zone of a commercial diffusion furnace. This furnace is in the form of a cylinder approximately $1 \mathrm{~m}$ long with a continuous through-passage through its length. A collimated airpath optical beam from a broad spectrum source (tungsten-halogen lamp) is focused on the crystal from outside one end of the furnace, with detection apparatus at the other end. This permits thermal isolation of the GaAs wafer, allowing its temperature properties to be measured. The furnace serves as an accurately controlled temperature chamber. Temperature can be directly incremented and held to close tolerances by control circuitry integral with the furnace. Temperatures from $200^{\circ} \mathrm{C}$ to more than $1000^{\circ} \mathrm{C}$ are easily obtained. For the purposes of this program, a temperature range from $200^{\circ} \mathrm{C}$ to $500^{\circ} \mathrm{C}$ was used.

The detection system at the exit beam consists of a standard laboratory monochromator (for determining wavelength) and a silicon avalanche detector, with associated electronics. A second detector monitors the entrance beam directly from the light source, so that small variations in lamp intensity due to voltage fluctuations and lamp aging can be accommodated. The monochromator is adjustable in one nanometer steps, and the photodetector output at each wavelength is recorded. The plot of detector output vs. wavelength, corrected for the silicon detector response, vields the transmission curve for the gallium arsenide crystal.

To speed up the process of experimentally measuring the absorption spectra, a direct interface from the experimental apparatus to a computer via an analog-to-digital converter was constructed. This eliminates the slow task of entering the data by hand. A $0.1 \%$ linear potentiometer is attached to the monochromator via gears and serves as a voltage divider. The voltage from the potentiometer is thus a direct function of the wavelength. Two channels of an eight-channel analog-to-digital converter are then fed the wavelength and optical intensity signals via coaxial cable, and the A-to-D output is accepted by an HP2100 computer system. The results are stored on magnetic tape via a Tektronix 4051 graphic terminal system. This procedure permitted an individual absorption curve to be obtained in a matter of a few minutes, including all data processing steps. 


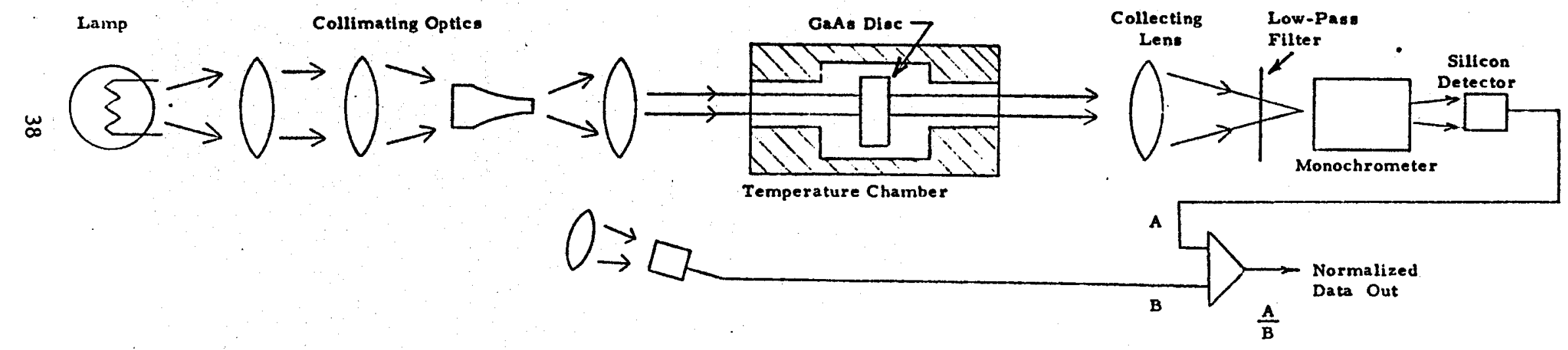

FIGURE 20. SCHEMATIC REPRESENTATION OF APPARA TUS FOR MEASUREMENT OF TEMPERATURE CHARACTERISTICS OF GaAs AS AN OPTICAL ELEMENT. 


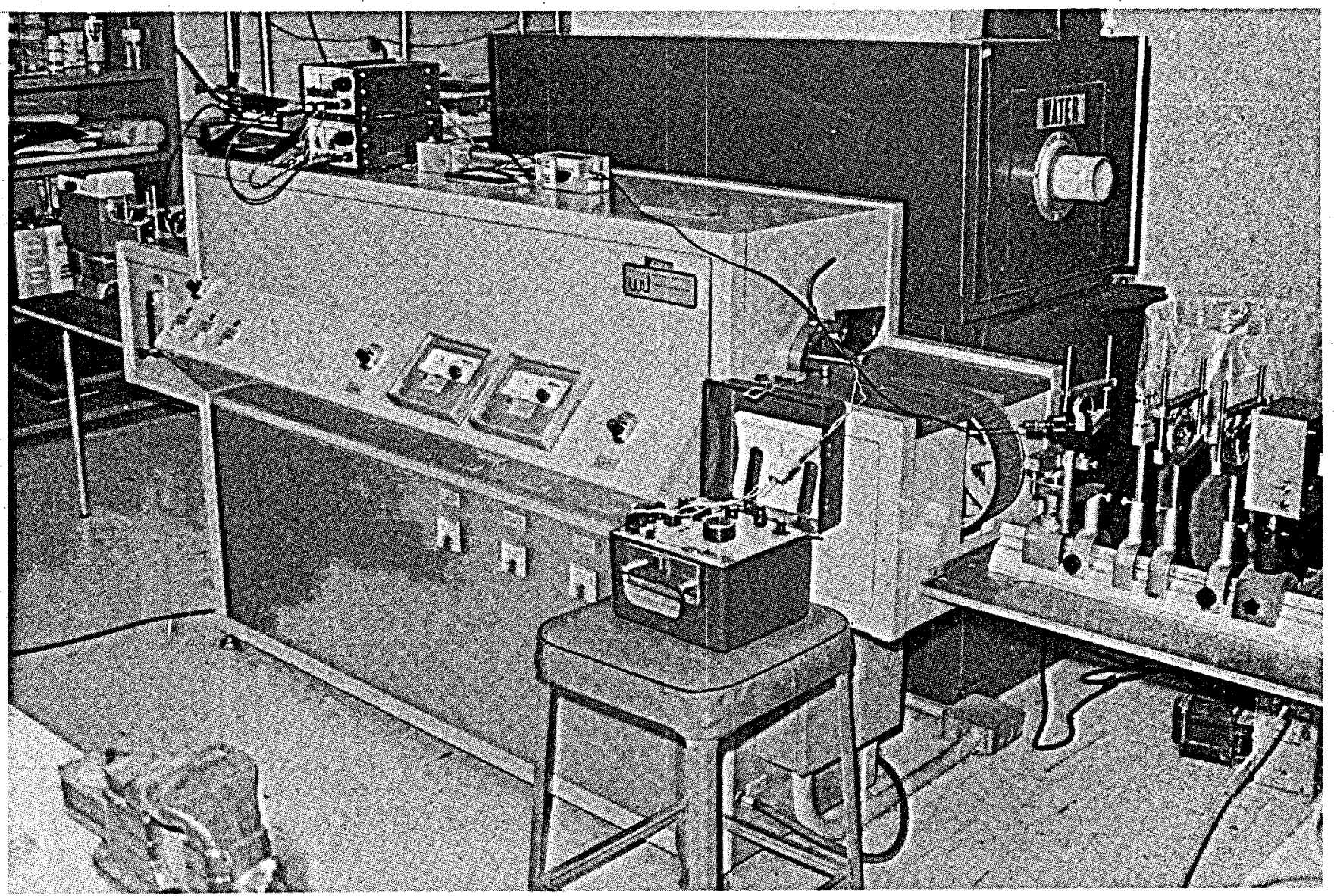

(a)

$\infty$
0
+1

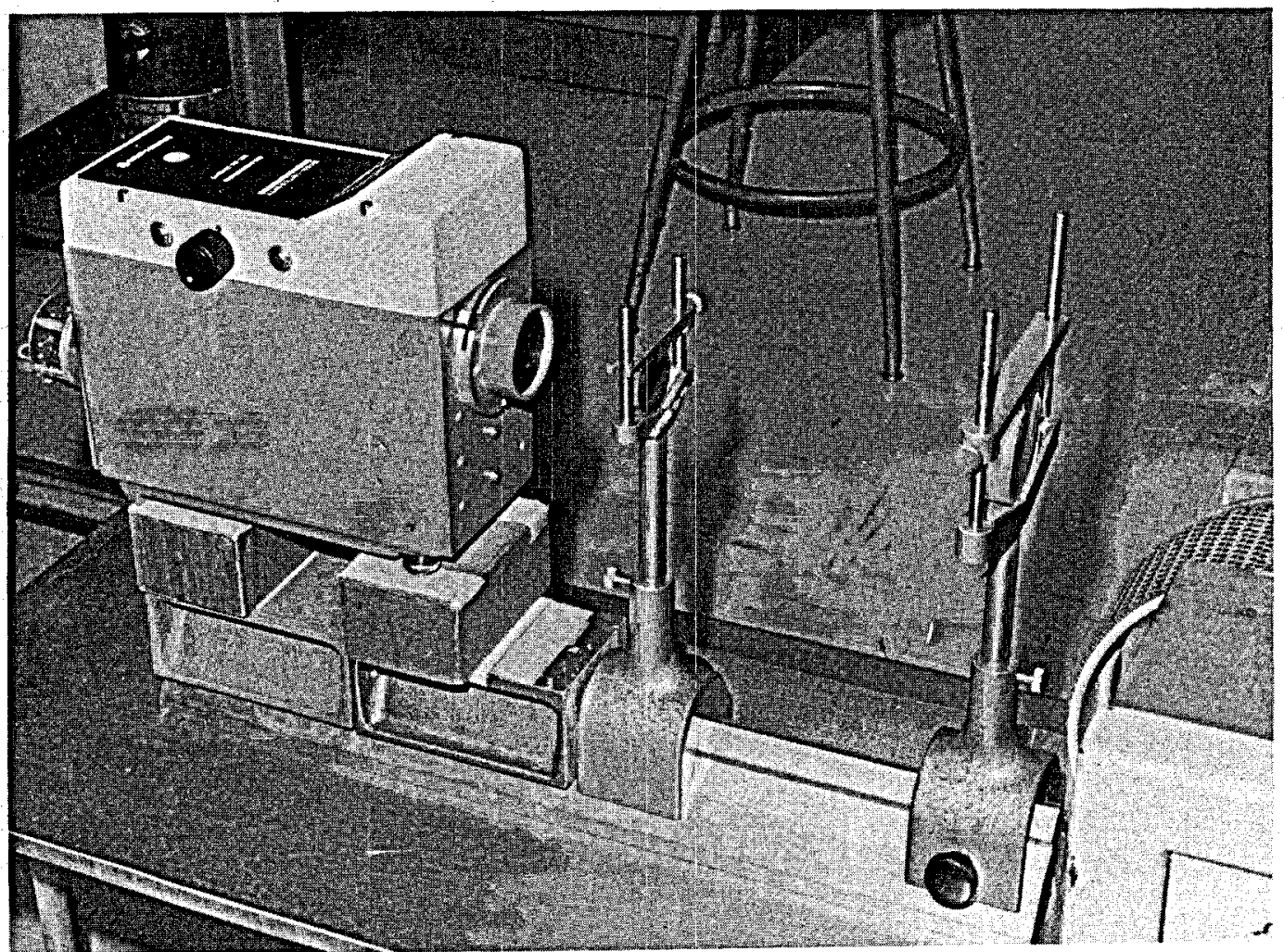

(b)

FIGURE 21。 EXPERIMENTAL A PPARATUS SHOWN SCHEMATICALLY IN FIGURE 20; a) overall view, optical source at right, b) monochromator. 39 
The initial set of experiments was performed with a crystal 15-mm-diameter and 2-mm-thick with an unknown dopant concentration. The normalized transmission spectra at high temperatures, corrected for detector response, are those shown in Figure 22. These data were then used to compute absorption coefficients and their wavelength derivatives by the method outlined in Appendix $A$. The results are shown in Figures 23 and 24.

As is evident from these Figures, the initial experimental results show that the absorption coefficient does indeed exhibit a well-defined point of maximum slope in the weak absorption tail. Furthermore, if $\lambda \mathrm{m}$ denotes the wavelength at the point of maximum slope and $1 / \lambda \mathrm{m}$ is plotted as a function of temperature, a linear relationship is obtained as illustrated in Figure 25. When this curve is expressed in energy units, rather than reciprocal wavelength units, we find that the slope is $-5.5 \times 10^{-4} \mathrm{ev} /{ }^{\circ} \mathrm{C}$, which is slightly larger, in absolute value, than the value of $\mathrm{dE}_{\mathrm{gap}} \mathrm{dT}$ reported in the literature (see Table I). Also, when a similar experiment was performed at room temperature, we found that the photon energy corresponding to $\lambda \mathrm{m}$ is $1.39 \mathrm{ev}$, which is slightly smaller than the band gap energy for pure crystals (1.43ev), as it should be. Thus, the room temperature value and the temperature dependence of the experimentally measured point of maximum slope are consistent with known properties of GaAs.

There are, however, two troublesome points concerning these absorption coefficient data. First, as can be seen in Figure 23, there is a very pronounced flattening of the absorption spectrum at short wavelength in marked disagreement with the results shown previously in Figure 19. One might attribute this to the fact that, with the relatively thick crystal used in our experiments, the transmitted intensity was extremely weak at short wavelengths, and it was therefore difficult to obtain reliable data. However, if this were the case, one would expect considerable scatter in the short wavelength data; instead, the flattening is very regular and systematic. This suggests that there may be some systematic error in the experimental or data processing procedures. If this is the case, and if the curve continues to rise sharply at short wavelengths, then one must question the data on the point of maximum slope. This is because in these initial experiments with a thick specimen, the point of maximum slope lies very near the flattened part of the spectrum. Its location, and, indeed even its existence, might therefore be determined by whatever causes the absorption curves to flatten at short wavelengths.

Although published studies of the effect of impurities on the absorption edge do not indicate that dopants can produce a point of maximum slope, ${ }^{(18)}$ the fact that impurity effects are not well understood, and the fact that we were working with a crystal with an unknown dopant concentration, led us to suspect that we might indeed be observing some previously unreported effect. At this point, therefore, we decided to obtain additional CaAs wafers with controlled dopant levels for further experimentation. These additional specimens were 12 -mil $(0.3 \mathrm{~mm}$-thick crystals of undoped CaAs, and crystals of the same thickness with zinc concentrations of $10^{18} \mathrm{~cm}^{-3}$ and $10^{19} \mathrm{~cm}^{-3}$.

Data on the absorption edge were obtained at room temperature with the crystal having a $10^{18} \mathrm{~cm}^{-3}$ acceptor concentration, and at temperatures from room temperature to $400^{\circ} \mathrm{C}$ with the other crystals. The room temperature data are shown in Figures 26 and 27. These results show that there is little difference between the absorption coefficients for the doped crystals, while the edge lies at shorter wavelengths for the pure specimen. This shift with dopant concentration is consistent with previously published data.

The disturbing point illustrated in Figure 26 is that even with thinner crystals at different dopant concentrations, we still observe a mysterious flattening of the absorption coefficient curves at short wavelengths. This time, however, there is considerable scatter in the short wavelength data which one can attribute to experimental difficulties in obtaining reliable data at low transmitted intensities. Still, the fact that the flattening occurs with all three dopant concentrations leads one to suspect that it is not impurity related. 


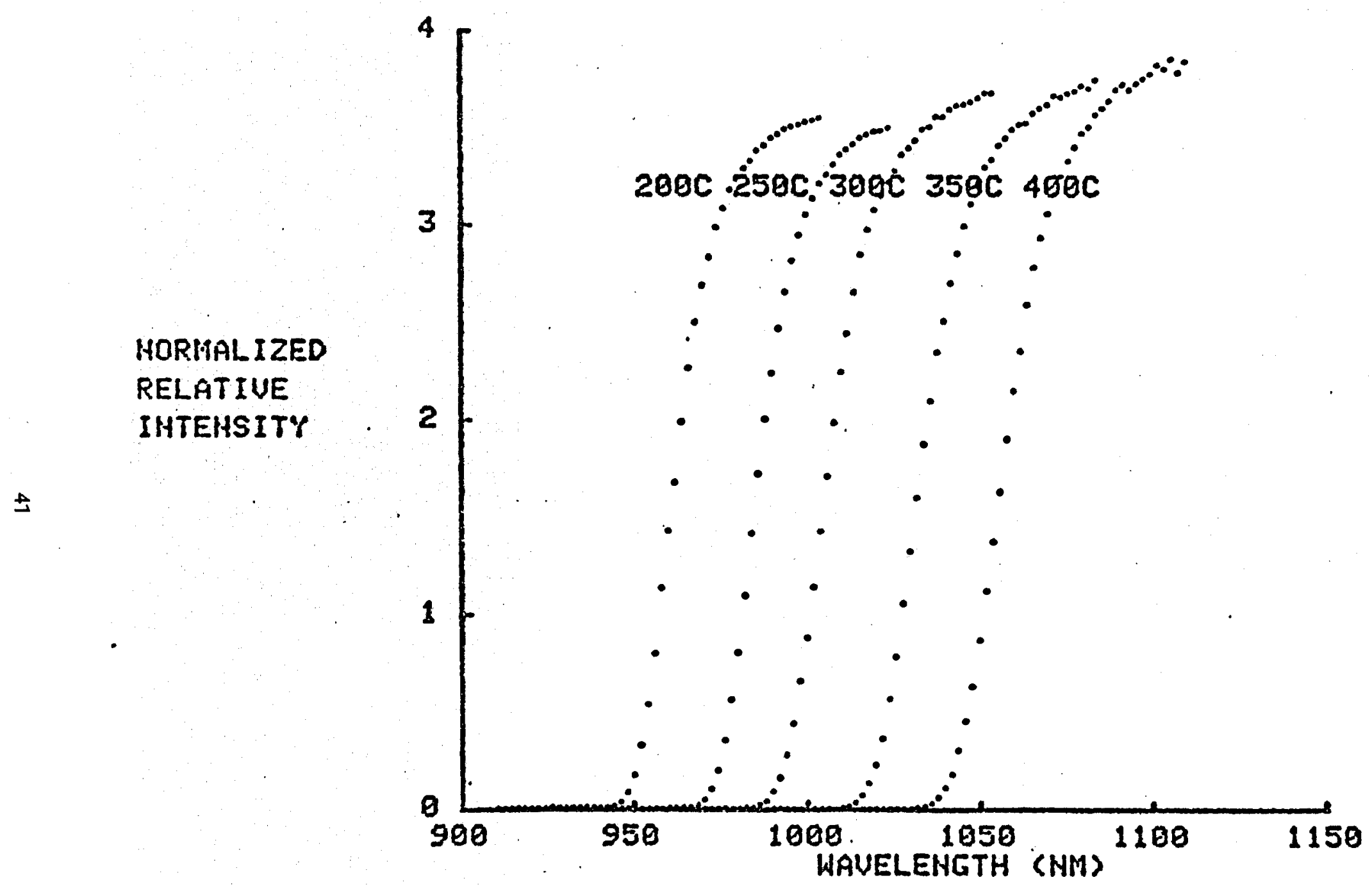

FIGURE 22. TEMPERATURE VARIATION DATA CORRECTED FOR SOURCE SPECTRUM FOR THE ORIGINAL GALLIUM ARSENIDE CRYSTAL 


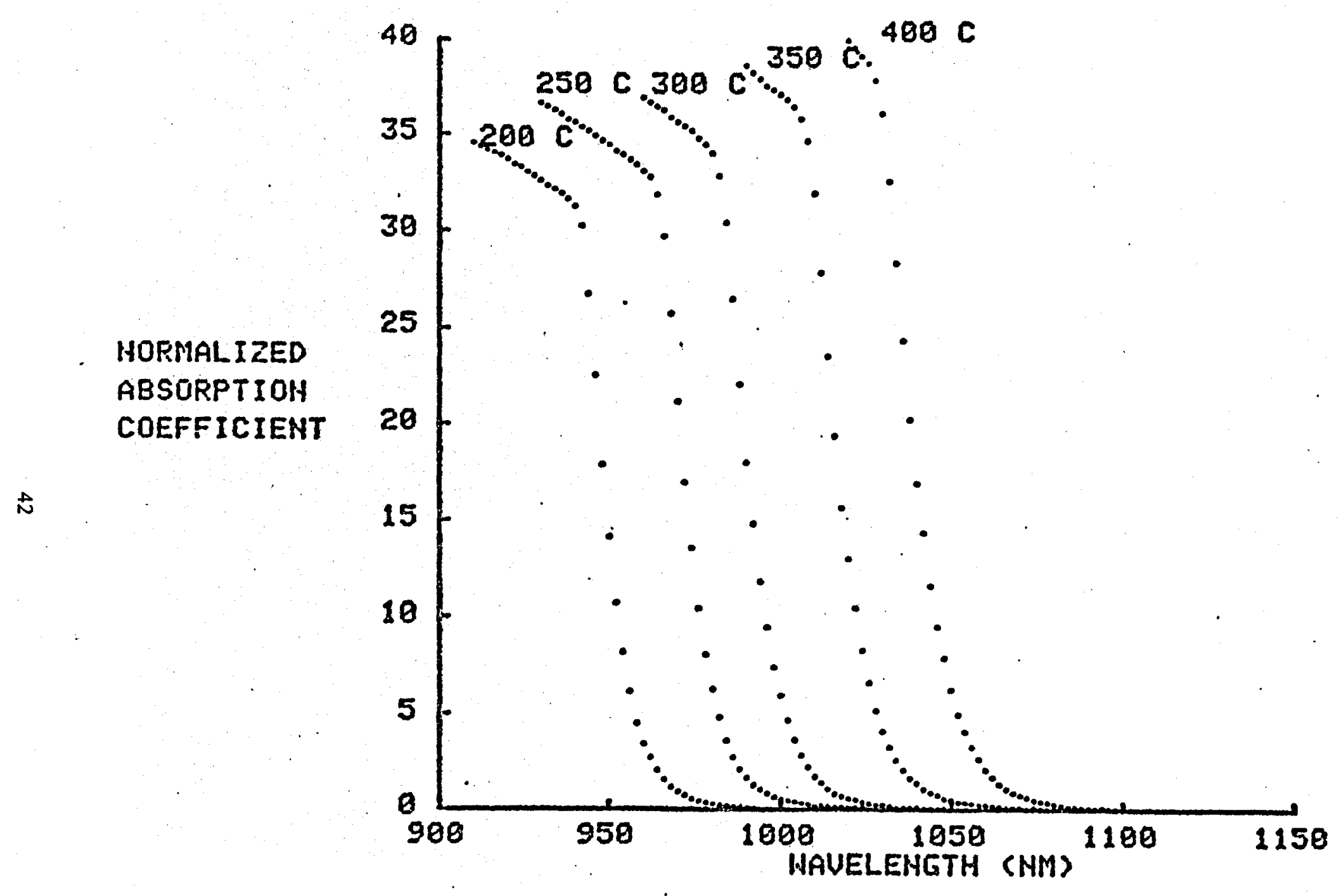

FIGURE 23. TEMPERATURE VARIATION OF THE ABSORPTION EDGE OF THE ORIGINAL GALLIUM ARSENIDE CR YSTAL 


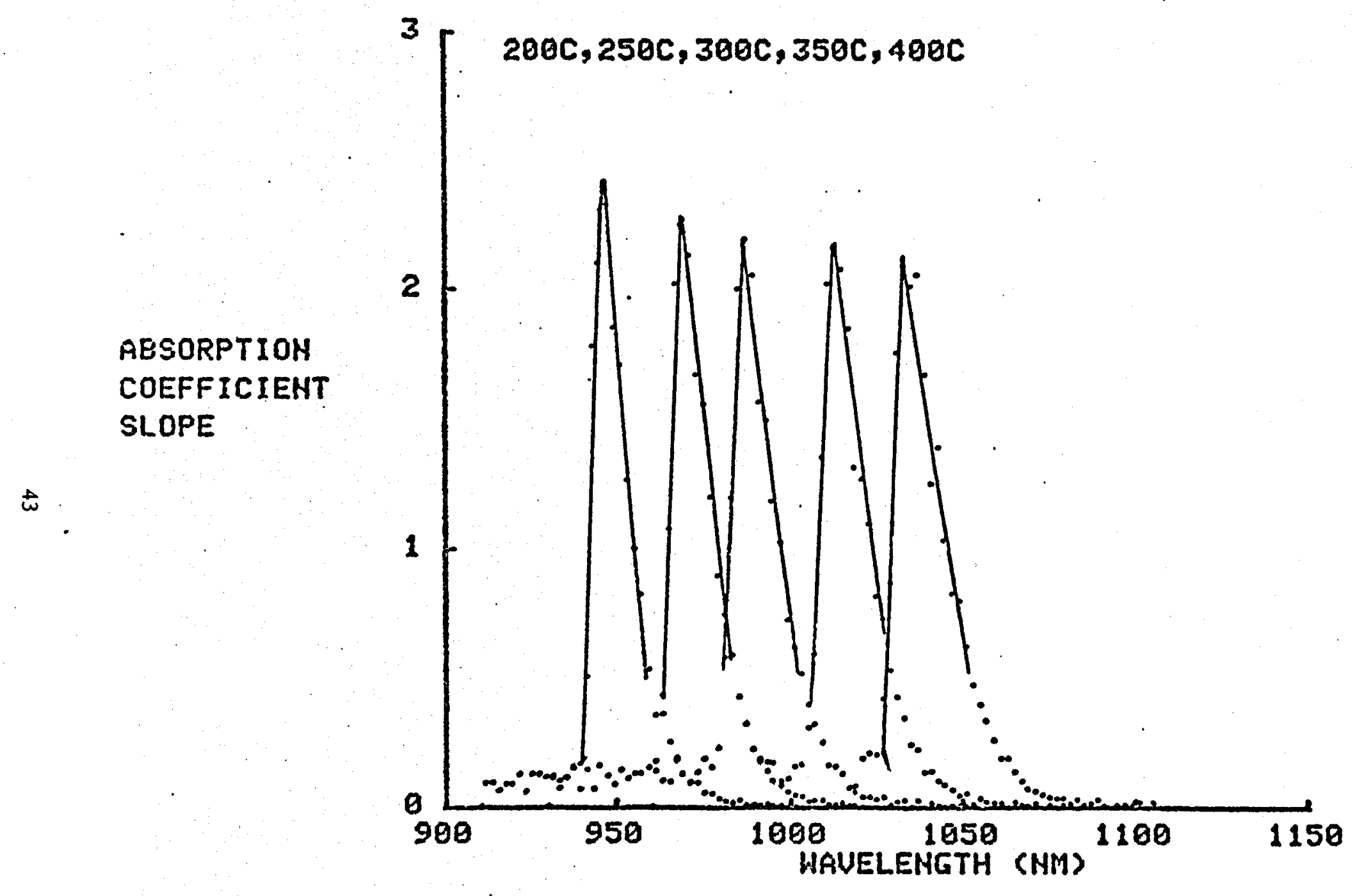

FIGURE 24. TEMPERATURE VARIATION OF THE ABSORPTION EDGE SLOPE OF THE ORIGINAL GALLIUM ARSENIDE CRYSTAL 


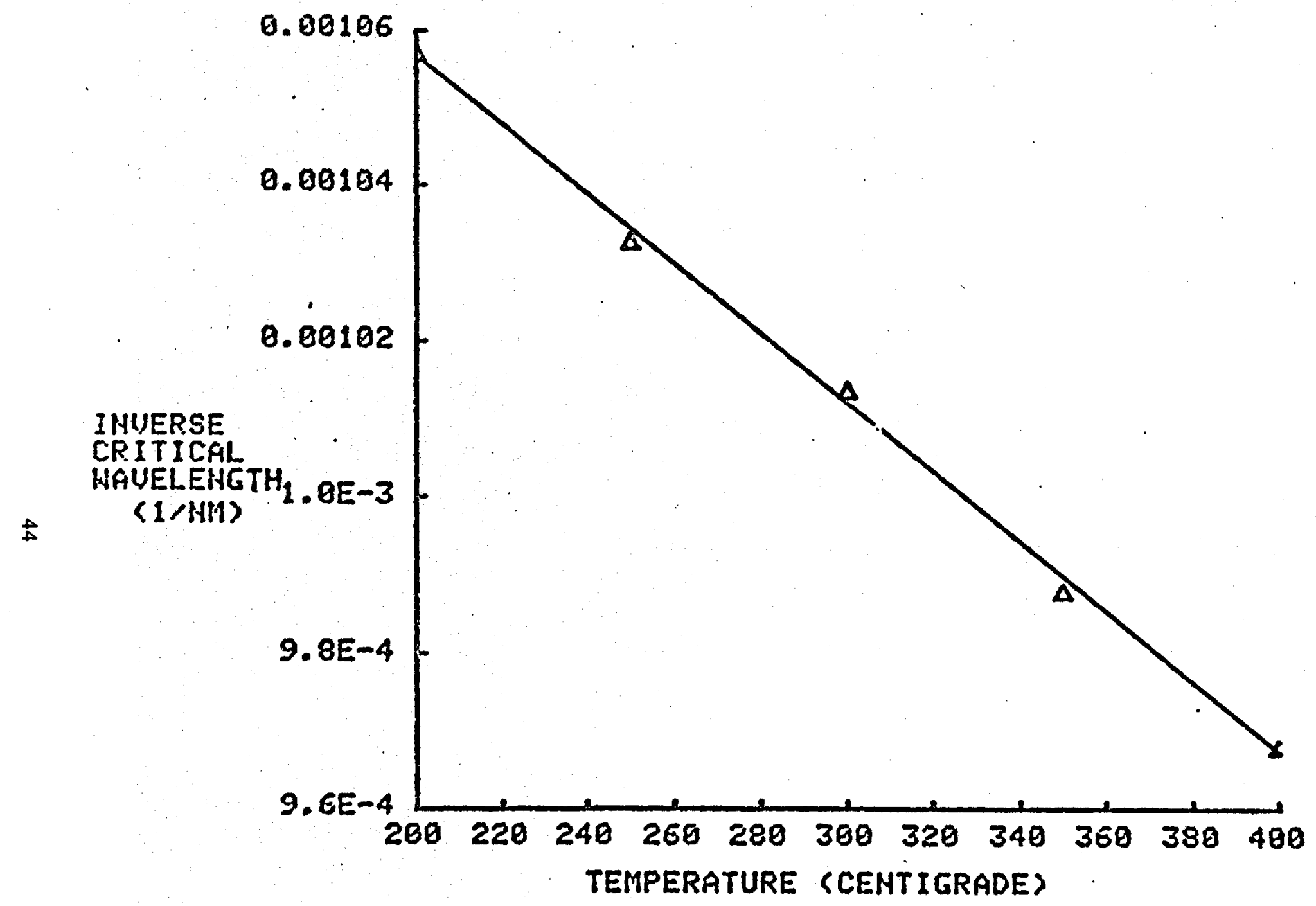

FIGURE 25. VARIATION OF INVERSE CRITICAL WA VELENGTH WITH TEMPERATURE FOR GaAs. Critical wavelength is the point at which absorption edge slope is maximum. 
FIGURE 26: ABSORPTION EDGE FOR (1) UHDOPED (2) 19+19.EC ZH DOPED AHD (3) 10 T18/CC ZN DOPED GAAS.TEMP $=230^{\circ}$

WAFER THICKNESS 12-Mil

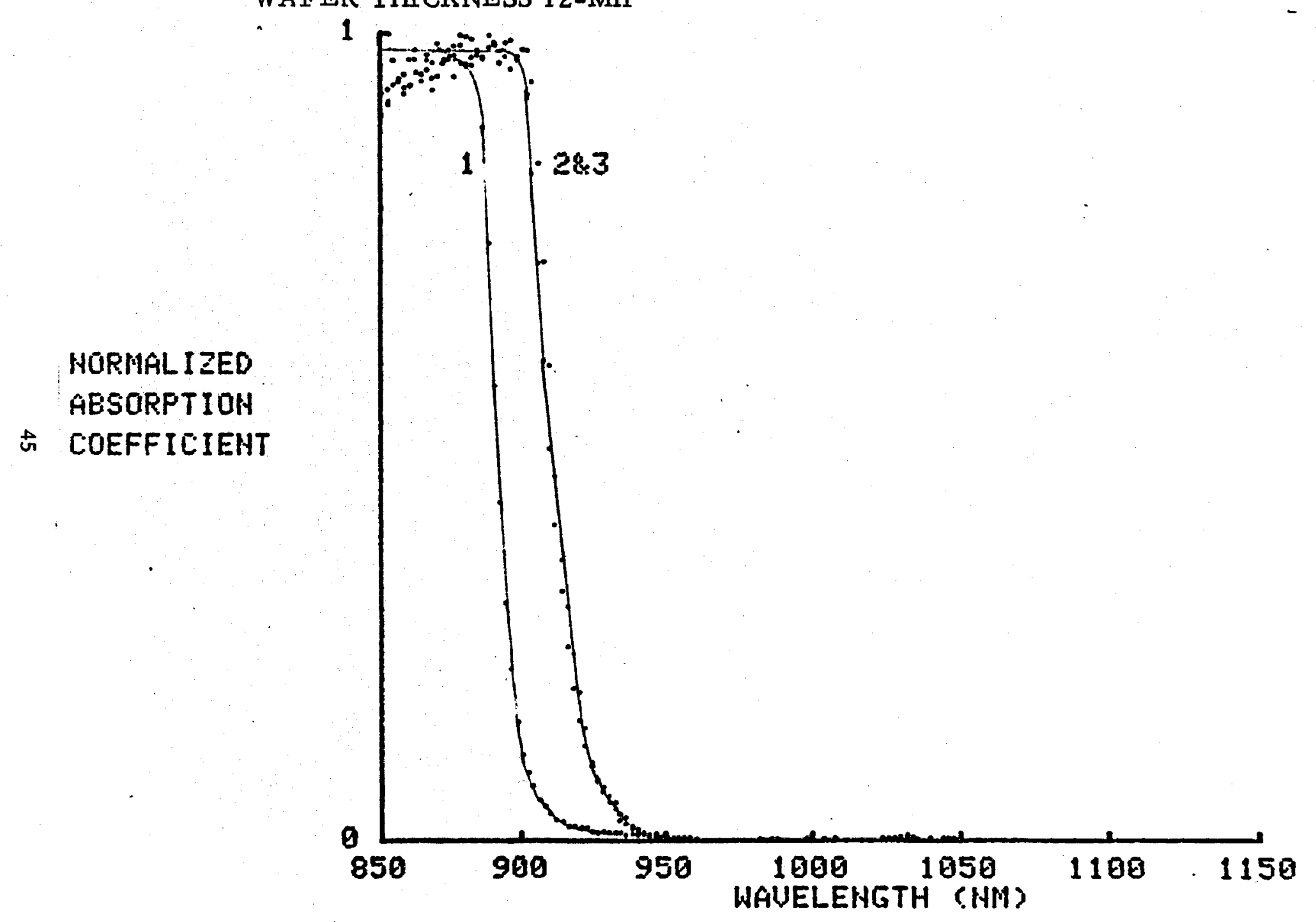


FIGURE 27: SLOPE OF ABSORPTION EDGE FUR (1)UMGOPED (2) 10419 GHD $93919+18$ ICC ZN DOPED GAAS.

WAF ER THICKNESS 12-Mil

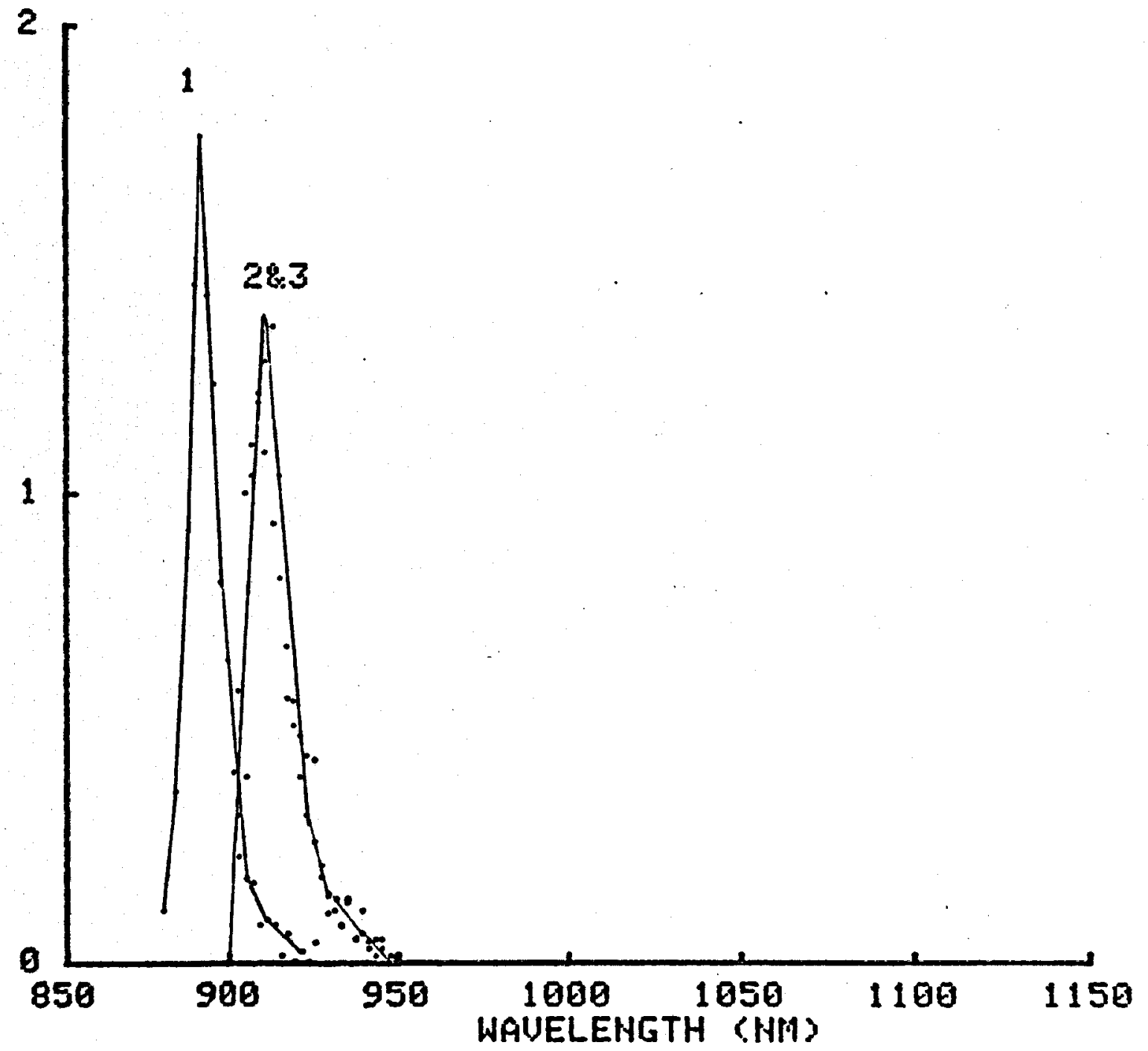


In a final attempt to explain these observations we performed still another set of experiments at room temperature with still thinner crystals. These specimens were prepared by mechanically polishing crystals with the three dopant concentrations noted above; thicknesses of about 2, 3, and 6 mils were obtained. A typical result, presented in Figure 28, again shows a flat absorption coefficient curve at short wavelengths.

At the present time, this short wavelength behavior of the absorption coefficient data is not explained. This is troublesome because it suggests that the experimentally determined shape of the short wavelength part of the absorption coefficient curve is somehow influenced by our method of measurement or data processing. If this is the case, then we cannot be certain that the observed point of maximum slope reflects some intrinsic property of gallium arsenide.

On the other hand, we have seen that the point of maximum slope consistently varies in the expected way with impurity content and, more importantly, with temperature. It is also insensitive to intensity fluctuations because only the slope, and not the magnitude, of the transmitted spectrum enters the temperature determination. Thus, while certain fundamental questions remain unanswered, we can at least tentatively conclude that the maximum slope method can be used with relatively thick (12 mil) crystals as a temperature measurement technique.

\section{The Photomultiplier "Threshold" Method}

Late in the program, another, approach to implementing the absorption edge method was pursued, which does not depend on the unresolved questions posed by the "maximum slope" method just discussed. This approach is based on the response characteristics of photomultiplier tubes at light levels near the minimum detectable intensity. As is well known, photomultipliers are designed to provide extremely large amplification (typically a factor of $10^{6}$ ) of the initial photocurrent produced by light incident on the photocathode of the device. Thus, when the intensity of incident illumination is increased from below the minimum detectable intensity to an intensity slightly above minimum, the output current increases from its noise level to a value orders of magnitude greater than the corresponding. change in the initial photocurrent. There is, therefore, an extremely narrow range of intensities at, and slightly above, the minimum detectable (threshold) intensity, within which the output current varies abruptly from its noise level to saturation.

In Figure 29 this threshold effect is illustrated. The curve at " $\mathrm{a}$ " might represent the intensity transmitted by a GaAs wafer. At short wavelengths, the crystal is almost totally opaque, and the transmitted intensity lies below the photomultiplier threshold. In this region the photomultiplier output current is constant, at a level representing dark current. As the wavelength is increased, a few photons are transmitted through the crystal, increasing rapidly with wavelength until the intensity crosses the threshold level of the photomultiplier at some point. Because of the extremely large photomultiplier amplification factor, and the fact that the transmitted intensity increases rapidly with wavelength, the output current rises abruptly at this point, almost as a step function, as illustrated in " $b$ " in the Figure. in fact, under optimum amplification conditions, the slope of this output curve is greater than the slope of the intensity spectrum by a factor of about $10^{6}$, the amplification factor of the photomultiplier. Then the wavelength at threshold can be determined easily and with great accuracy by simply noting the wavelength at the on-off transition. Because the temperature dependence of the threshold wavelength is nearly the same as that of the band gap wavelength (this is demonstrated in Appendix A), the threshold can be used as the reference point for temperature sensing.

Although the threshold method does not require a measurement of transmitted intensity, it is clear from Figure 29 that the location of the threshold is influenced by intensity fluctuations. In a practical well-logging system such fluctuations will occur, and will constitute the principal uncertainty in the determination of temperature. 


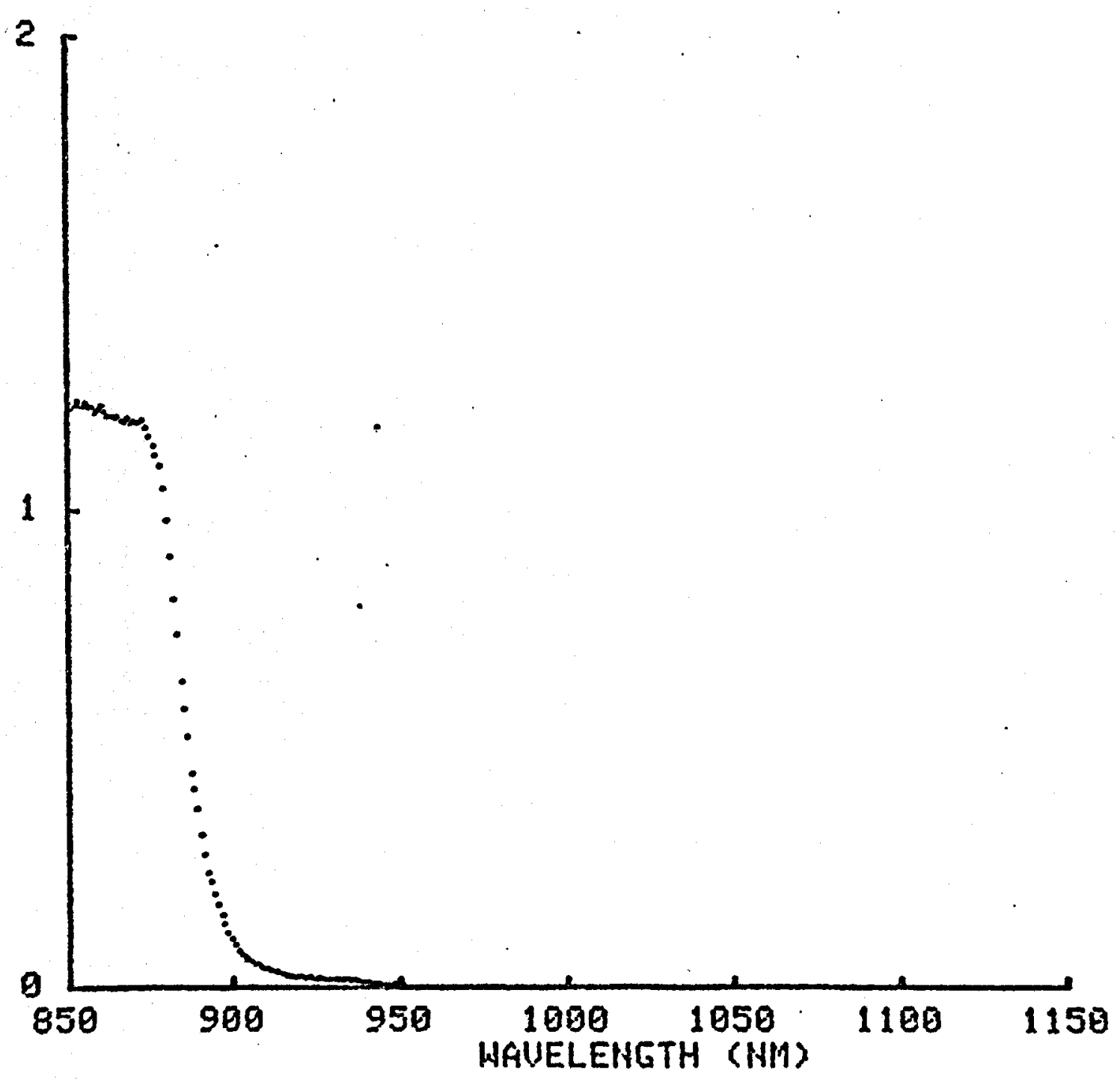

FIGURE 28. ABSORPTION EDGE FOR UNDOPED GaAs WAFER THICKNESS 3-Mil 


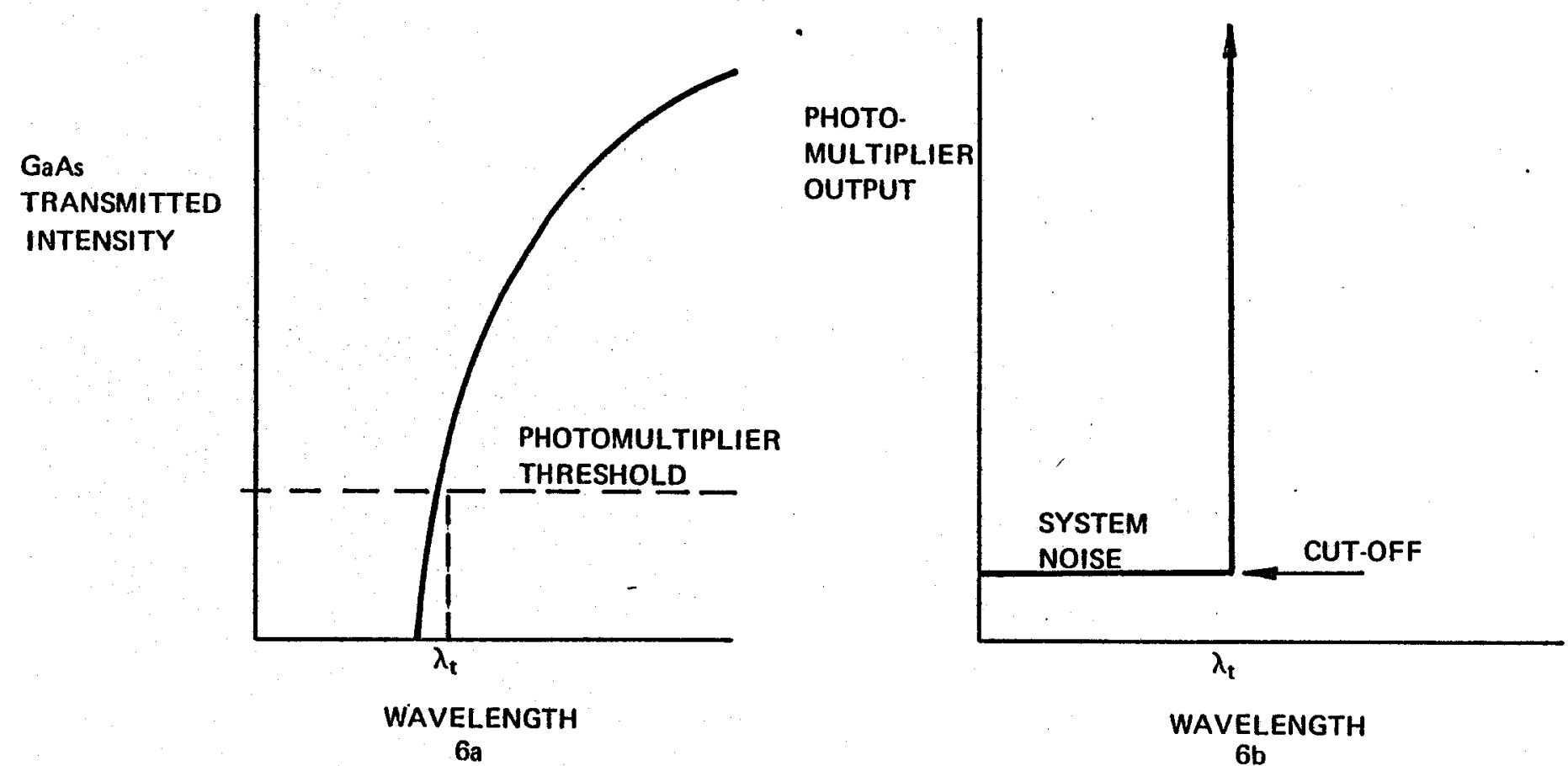

FIG 29.PHOTOMULTIPLIER-THRESHOLD MÉTHOD OF DETERMINING CUT-OFF

WAVELENGTH FOR GaAs

a) TRANSMITTED INTENSITY OF LIGHT THROUGH GaAs NEAR ABSORPTION EDGE

b) TRANSMISSION CURVE AS MULTIPLIED BY PM AMPLIFICATION FACTOR 
To investigate the severity of this problem, both analytical and experimental studies of the influence of changes in intensity on the threshold wavelength were performed. Details of the analytical work are reported in Appendix $A$ and experimental results are reported in the next section. Both the calculations and experiments indicate that as long as the threshold is measured near cutoff (where the transmitted intensity varies rapidly with wavelength), intensity variations of rather large magnitude cause very small changes in the threshold wavelength. For example, calculations in Appendix A show that, in a typical example in which transmitted intensity is $20 \%$ of maximum intensity, an intensity increase by a factor of 50 will produce an apparent wavelength shift of only $3 \mathrm{~nm}$. This is equivalent to an error in temperature measurement of about $7.5^{\circ} \mathrm{C}$.

It should be noted that if the photomultiplier threshold under the same conditions were higher, so that the threshold intensity occurred at a point where the slope is less, then poorer accuracy would result. It is therefore important to accurate temperature sensing by this method that the system be optimized for maximum transmitted intensity and good low-noise photomultiplier performance. One needs a high intensity source, low-loss fibers, efficient fiber coupling, and, probably, sensor elements as thin as possible.

\section{Experimental Evaluation of Threshold Method}

The experimental apparatus used in testing the threshold approach is illustrated in Figure 30 . The source for most measurements was an 18 watt tungsten lamp which was coupled to a fiber by simply placing the end of the fiber against the bulb. The sensor in this series of experiments was the actual prototype device described in more detail in Section A.7, following. It consists of a 12 mil thick GaAs wafer coupled to two fibers, one of which carries light from the source to the crystal, while the other carries the transmitted signal to the detection system.

To remove the extraneous light propagating in so-called cladding modes in the short fibers (these modes are attenuated and therefore will not be present in a long optical cable), about eight inches of the buffer coating was stripped from each of the two pieces, and the stripped sections bent into a " $U$ ", immersed into containers of glycerin. The index of refraction of glycerin nearly matches that of the fiber cladding, and propagation around the bend by multiple internal reflections at the glycerine-cladding interface is thus eliminated.

The detection system consisted of a lens to focus the light from the output end of the fiber on the slit of a monochromator, a filter to block visible radiation, the monochromator itself, and, finally, the photomultiplier and associated electronics. The actual output signal was the voltage across a load resistor in the photomultiplier anode circuit, filtered and displayed on an oscilloscope.

The photomultiplier tube used was an RCA 31004 , equipped with an S-1 photocathode for infrared detection. This tube is generally operated with cooling to liquid nitrogen temperature to reduce noise, resulting in a noise reduction of about a factor of $10^{6}$ as compared with dark current at room temperature. In these experiments, however, no satisfactory method for coupling the output of the monochromator to the photomultiplier in a cooling chamber was available, and the detector was operated at room temperature. As a result, the detection system is far from optimum, and significant improvements in detection sensitivity are therefore realizable.

The initial set of experiments was performed with the GaAs at room temperature. The procedure was to first set the monochromator at a short wavelength where the crystal is opaque. The monochromator wavelength was then increased in 1-nm increments, until the first indication of a transmitted signal was noted on the scope. The corresponding wavelength was taken to be the threshold wavelength defined above.

Once these checkout experiments were completed the sensor was placed in an oven and the procedure repeated at several temperatures up to $250^{\circ} \mathrm{C}$, which was the highest temperature attainable with 


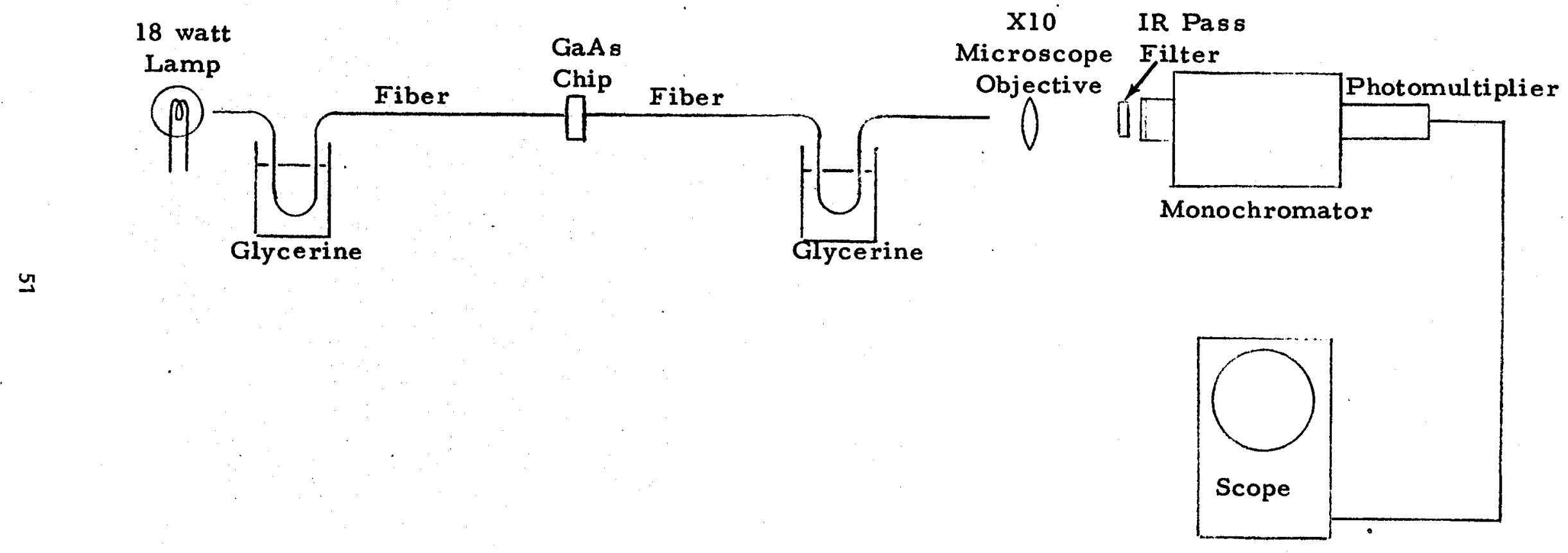

FIGURE 30. EXPERIMENTAL ARRANGEMENT FOR MAKING PHOTOMULTIPLIER THR ESHOLD MEASUREMENTS WITH GaAs TEMPERATURE SENSOR 
the temperature chamber available. (The $275^{\circ} \mathrm{C}$ data were later added from separate, spot measurements.) To check on the repeatability of the threshold data, experiments were performed over a two-day period with several heating and cooling cycles. At one point the apparatus was even disassembled and then re-assembled.

The results of these tests are shown in Figure 31 which is a plot of the observed threshold wavelength as a function of temperature. The small amount of scatter evident here is thought to be caused by a lack of thermal equilibrium between the GaAs and the oven thermometer, particularly at high temperature where the stability of the oven was poor. Overall, however, the results are remarkably consistent, demonstrating the reliability of the threshold method.

To investigate sensitivity to intensity fluctuations, we replaced the 18 watt lamp, and increased the measured brightness. The threshold determination was then repeated at room temperature. These results are summarized in Figure 32, showing the wavelength shift at several values of input intensity. The values agree closely with the calculated results. Even if no corrections for these large intensity changes are incorporated in data processing, the error in temperature measurement corresponding to an $18 \mathrm{~dB}$ increase in attenuation is only $10^{\circ} \mathrm{C}$. Practically, this represents a very severe intensity fluctuation, and much better accuracy should be realized in practice.

As a result of these experiments, we conclude that it is possible to apply the photomultiplier threshold method to temperature logging with semiconductor temperature sensors. The technique is simple, requires minimal data processing and should, therefore, lend itself well to automatic operation. The principal difficulty with the approach is that, in order to insure high accuracy and insensitivity to intensity fluctuations, the transmitted intensity must be as large as possible, and the photomultiplier detection threshold as low as possible. Whether this will pose serious design problems remains a question for further study.

\section{Prototype Temperature Sensor Design and Fabrication}

Once the basic questions regarding the intrinsic capabilities of an optical temperature sensor were resolved, the remaining task was the design of a practical assembly including coupling the input and output fibers to either side of a GaAs wafer. There are two important requirements that the coupler must meet. It must, first of all, provide very accurate alignment of the fibers, as even a slight misalignment can produce serious coupling losses. Furthermore, because the coupler must function at high temperatures, its structural integrity, including fiber alignment, must be maintained throughout the full temperature range. These constituted the principal objectives of the prototype design effort.

a. Initial Approach. The first design concept is illustrated in Figure 33. The GaAs active temperature sensor element was made in the form of a small cylindrical pellet, cored from a polished wafer with proper thickness. An individual pellet approximately $0.1 \mathrm{~mm}(0.004$-in.)-diameter was cemented between the prepared ends of two optical fibers with high-temperature adhesive, assembled inside a $0.15 \mathrm{~mm}$ ID stainless steel tube. The complete assembly had the appearance of a short stainless rod, about the size of a sewing needle a few millimeters in length, from which the two optical fibers emerged.

Two of these units were constructed. Aside from the difficulty of aligning the fibers inside the tube with sufficient accuracy, the high-temperature epoxy used in the assembly was found to fracture at elevated temperatures due to differential temperature expansion. After several unsuccessful attempts to substitute other adhesives, this approach was abandoned.

b. Prototype Design. The second design is based on a precision metal fixture into which the individual fibers and a small GaAs wafer are positioned and cemented in place. 


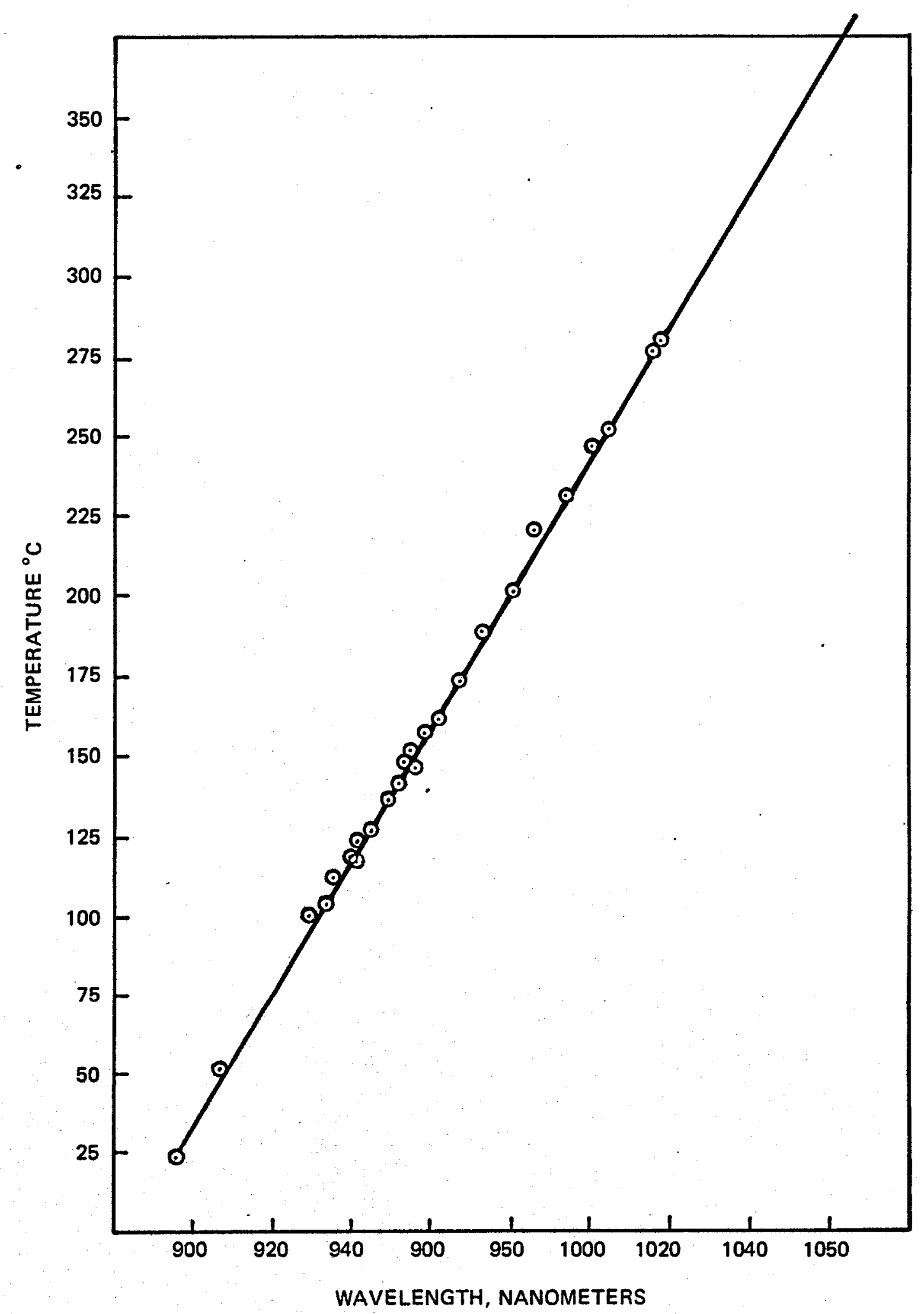

FIG 31.EXPERIMENTALLY DERIVED TEMPERATURE CALIBRATION FOR GaÁs OPTICAL THERMOMETER 


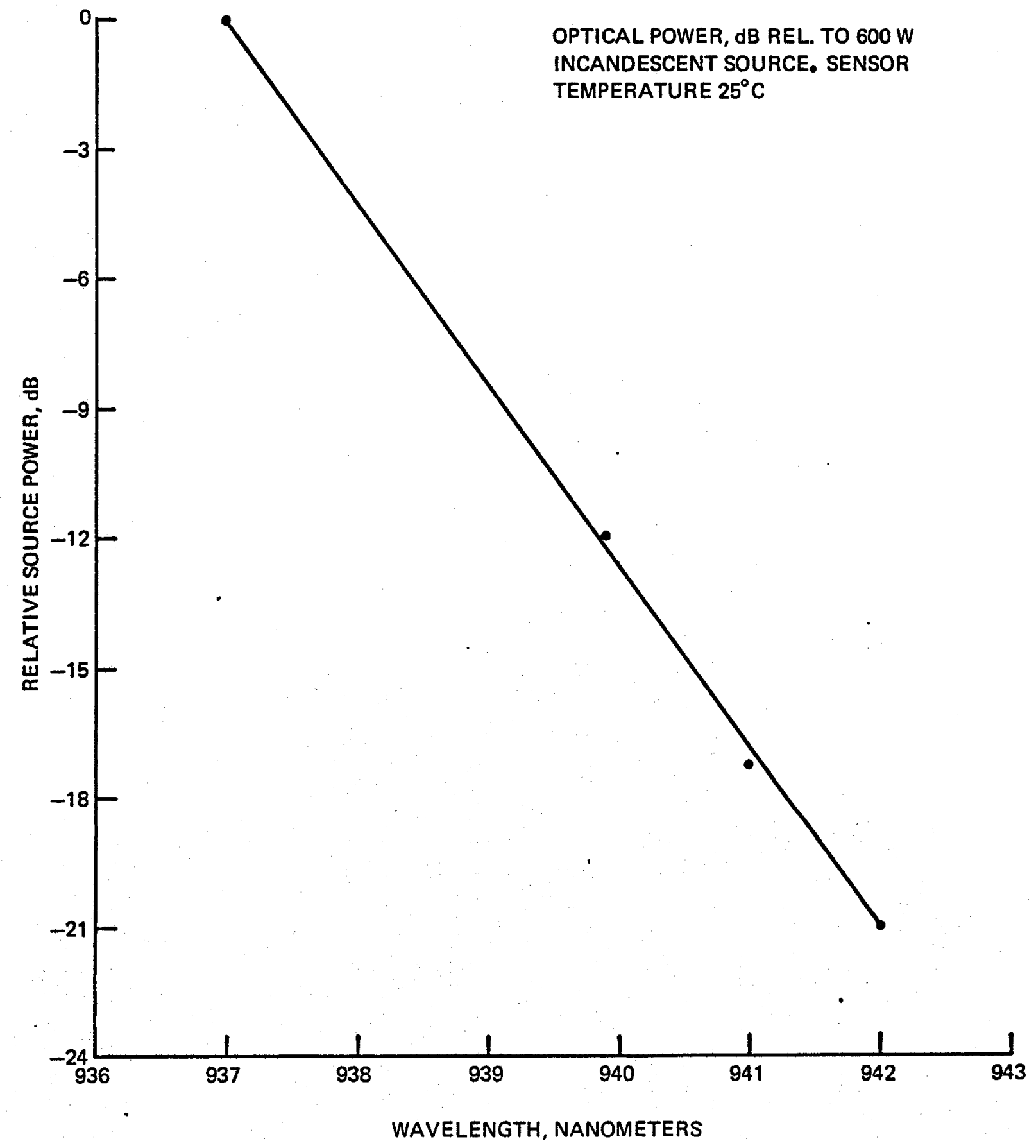

FIGURE 32. APPROXIMA TE EFFECT OF TRANSMITTED OPTICAL POWER ON MEASURED CUTOFF WAVELENGTH, GaAs TEMPERA TURE TRANSDUCER. 


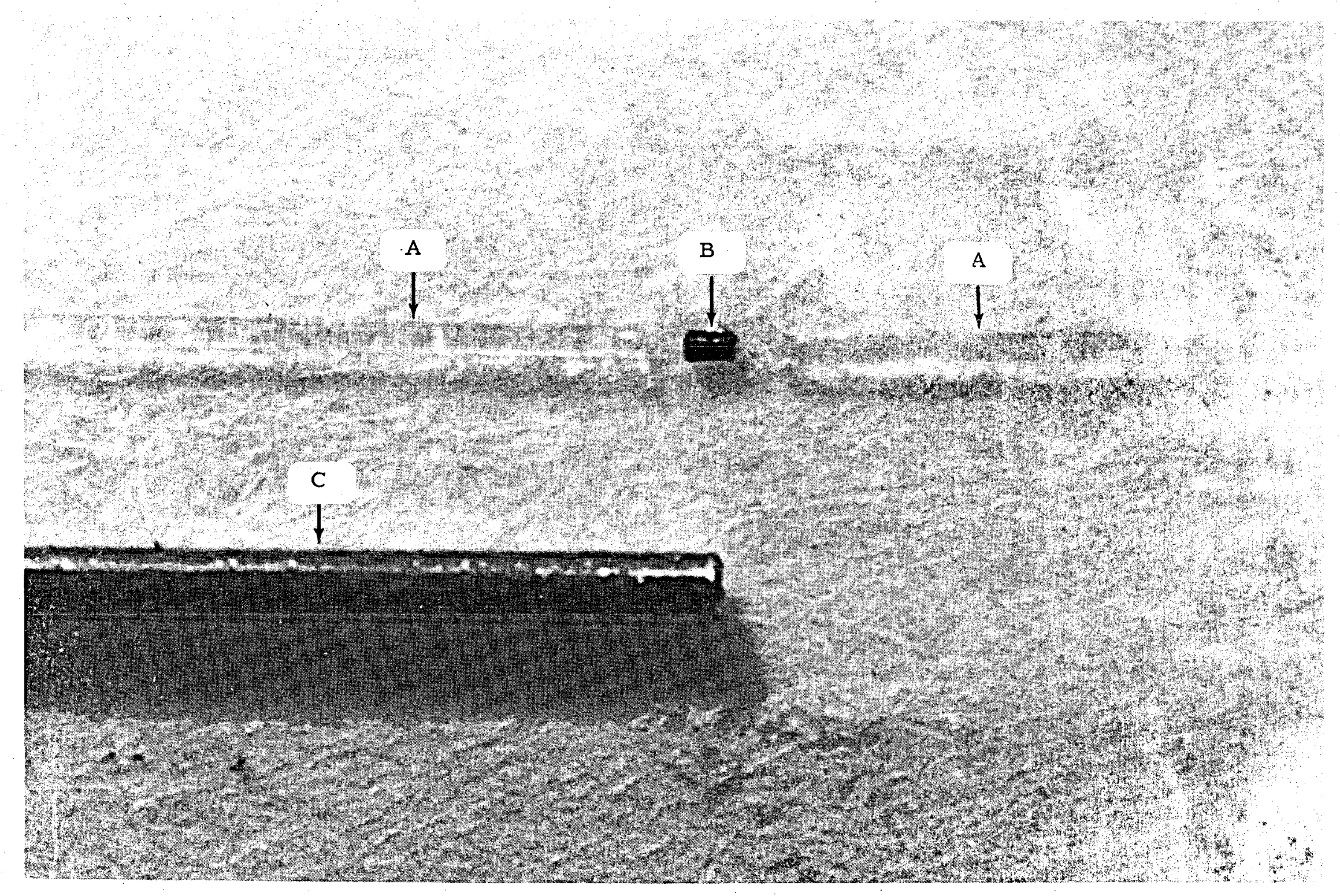

FIGURE 33. COMPONENTS OF FIRST PROTOTYPE GaAs TEMPERATURE SENSOR PRIOR TO ASSEMBLY. (A) Optical fibers; (B) GaAs pellet; (C) Stainless steel tubing. The GaAs sensor element is approximately .004 in. diameter by $.012 \mathrm{in}$. This design did not perform satisfactorily, because of bonding difficulties inside the tube. 
The precision fixture is a milled metal cylinder approximately 1.5 -mm-diameter, shown in the sketch in Figure 34. First the axial slot is cut, and a length of stainless steel tubing cemented through the length with high-temperature epoxy. After the adhesive is set, a second perpendicular slot is milled across the fixture, cutting through the cemented tubing. This slot is designed to hold a prepared GaAs sensor element.

The assembly is completed by inserting the GaAs wafer into the cross slot, and two optical fibers into the center tube, one in each end, facing the wafer. The fibers are carefully aligned and the complete assembly is given a coat of Dow Corning 804 silicone resin, and air dried. The resin is cured in a hightemperature curing cycle $\left(250^{\circ} \mathrm{C}\right.$ or higher) after the material has set.

This construction technique has been successful. The elements have remained intact through the curing cycle and several oven test cycles at temperatures as high as $300^{\circ} \mathrm{C}$. The calibration data presented in the previous section of this report (Figure 31) was acquired with the device, shown in Figure 35.

Although the sensor functions well and shows predicted temperature response, the transmission efficiency is lower than anticipated. The overall attenuation of the sensor compared to an equal length of uncut fiber is approximately $24 \mathrm{~dB}$.

Part of this loss was anticipated from earlier experiments. For example, in Figure 36 is shown the experimentally measured transmitted spectrum for two coupled fibers under various conditions. The upper curve is the intensity.transmitted when the prepared ends of the fibers are in contact (in air) and after lateral adjustments to maximize transmission (this represents approximately a $1.0 \mathrm{~dB}$ loss compared to an unbroken fiber). The center curve was obtained when the fiber ends were separated by a distance equal to the crystal thickness $(0.3 \mathrm{~mm})$. Comparison of these two curves shows that the loss caused by beam spreading in the $0.3 \mathrm{~mm}$ air gap between the fibers is about $3.1 \mathrm{~dB}$, compared to smoothly butted fibers.

The lower curve results when a GaAs wafer is inserted in the same gap between the fibers. At wavelengths longer than the absorption edge, the effect of the transparent crystal still shows a substantial loss (an additional $8.8 \mathrm{~dB}$ at $1000 \mathrm{~nm}$ ), which may be attributed to multiple reflections and spreading. This loss is larger than would be expected from the theoretical expression for multiple reflection loss where, if one assumes the reflectivity of GaAs is 0.48 as determined by the method outlined in Appendix $A$, then the theory for a plane parallel beam gives $4.5 \mathrm{~dB}$ as the multiple reflection loss, compared to the experimentally determined $8.8 \mathrm{~dB}$. Evidently, the plane wave theory is inadequate and one must consider the combined effects of beam spreading during multiple reflections to account for the observed loss. The analysis of this combined loss mechanism has not yet been attempted.

The total loss caused by fiber end separation and multiple reflections of the divergent beam within the GaAs is thus seen to be about $12 \mathrm{~dB}$ at wavelengths longer than the absorption edge. To this might be added about $3 \mathrm{~dB}$ to account for the so-called intrinsic losses (core diameter and index of refraction mismatch, reflections at the fiber ends and other, less important losses) giving a total of about $15 \mathrm{~dB}$, compared to the $24 \mathrm{~dB}$ total losses observed. This leaves about $9 \mathrm{~dB}$ that must be attributed to alignment errors and other causes in the prototype sensor.

Although great care was taken to insure proper fiber alignment before the device was cemented with silicone resin, it is possible that the alignment was upset during the drying and curing process. To see how this might affect performance, additional experiments were conducted to estimate the losses incurred by lateral (radial) alignment errors and by fiber end separation. The results, presented in Figures 37 and 38 , show that a very small lateral misalignment could account for the additional $9 \mathrm{~dB}$, while a large additional axial separation (of the order of $300 \%$ of the fiber diameter) must be assumed if the loss is to 


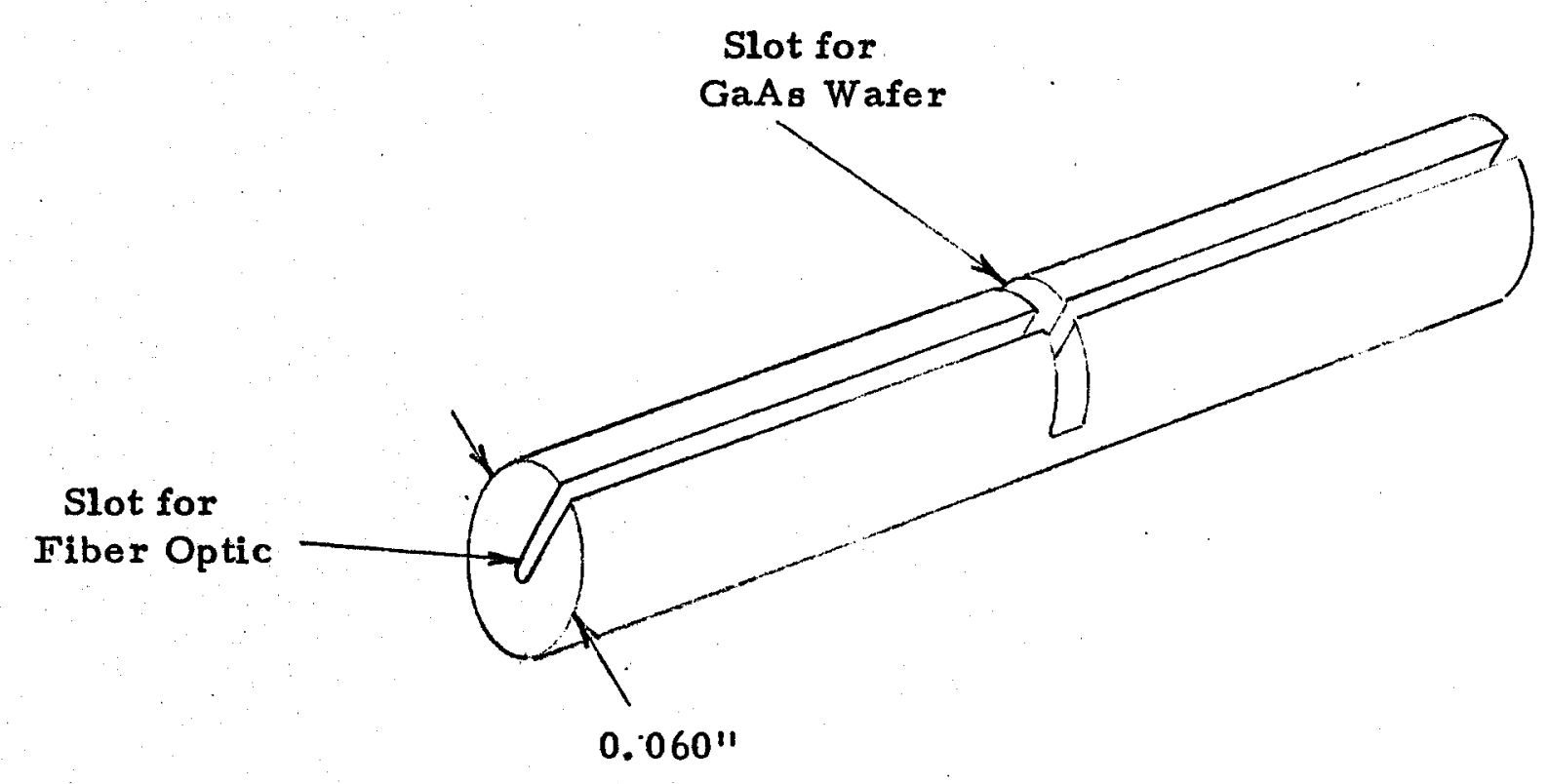

FIGURE 34. COUPLING ELEMENT FOR SECOND PROTOTYPE OPTICAL TEMPERATURE SENSOR. 


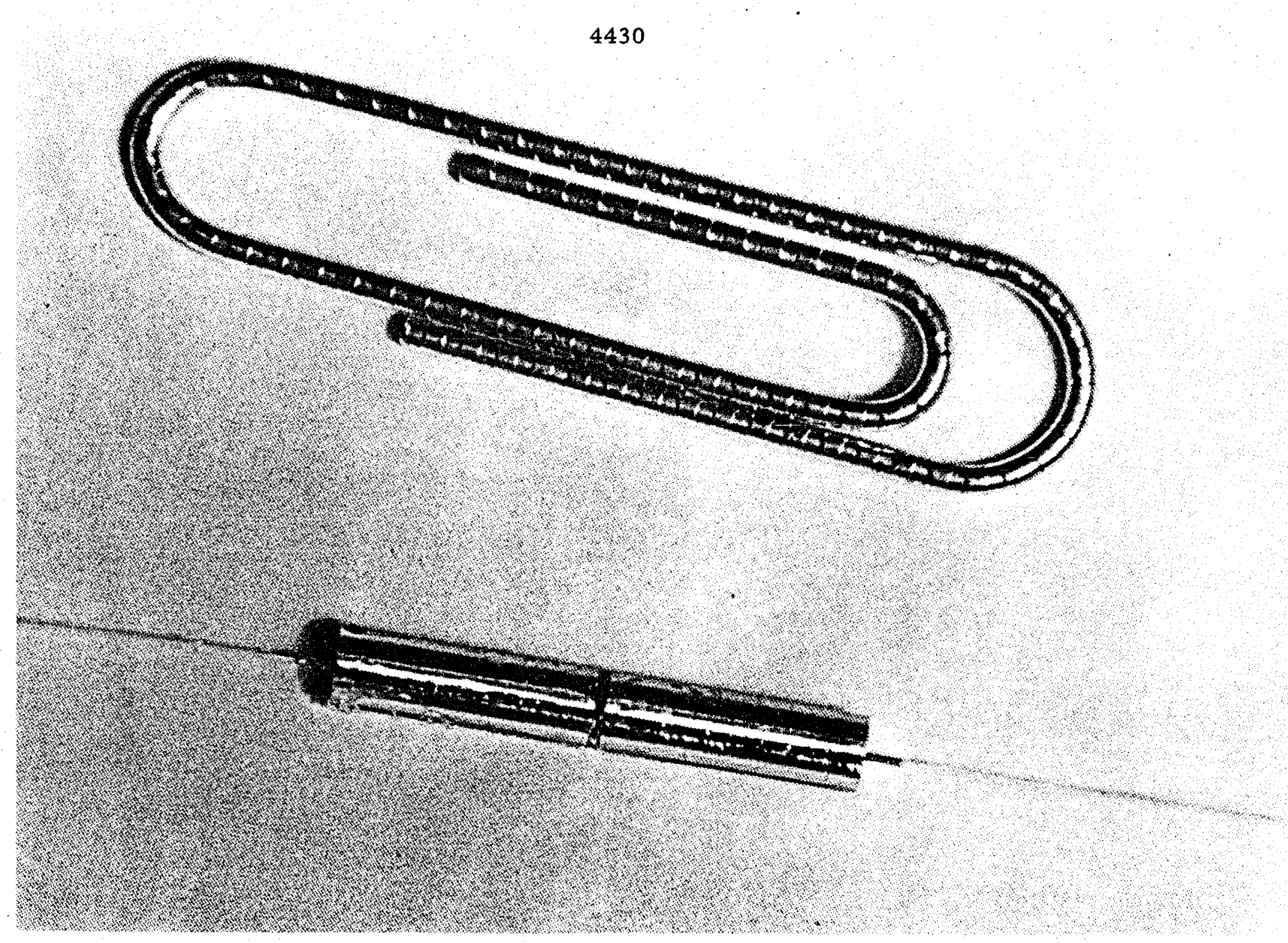

FIGURE 35. PROTOTYPE OPTICAI TEMPERATURE SENSOR FROM WHICH REPORTED WAVELENGTH-TEMPER ATURE DATA WERE OBTAINED. (APprox. 6x) 
LOSS, dB RE: UNBROKEN FIBER

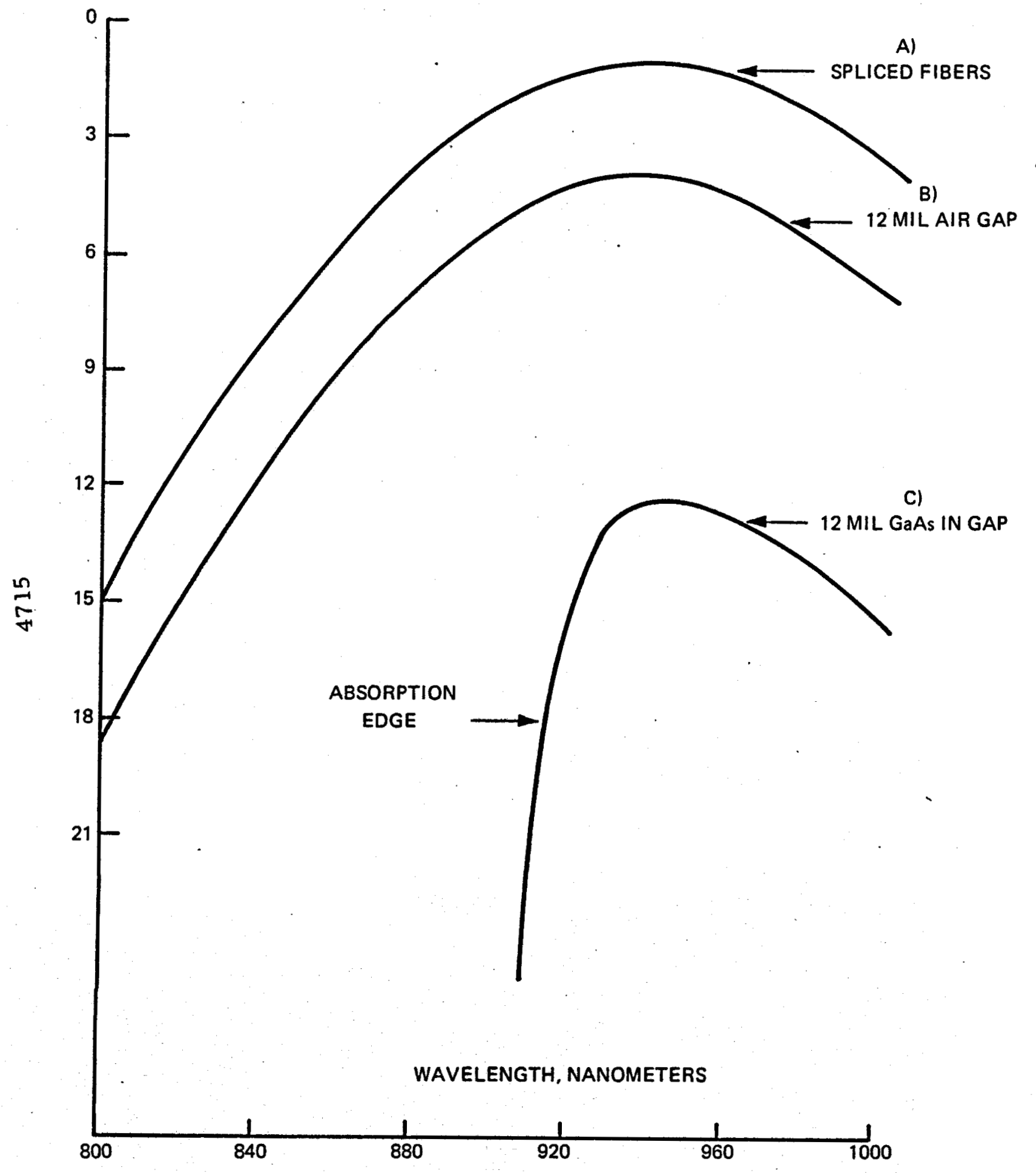

FIGURE 36. TRANSMISSION SPECTRA OF TWO SPLICED, ALIGNED FIBERS a) no gap between ends, b) $12-\mathrm{mil}(0.4 \mathrm{~mm})$ air gap, and c) 12-mil GaAs wafer in gap 


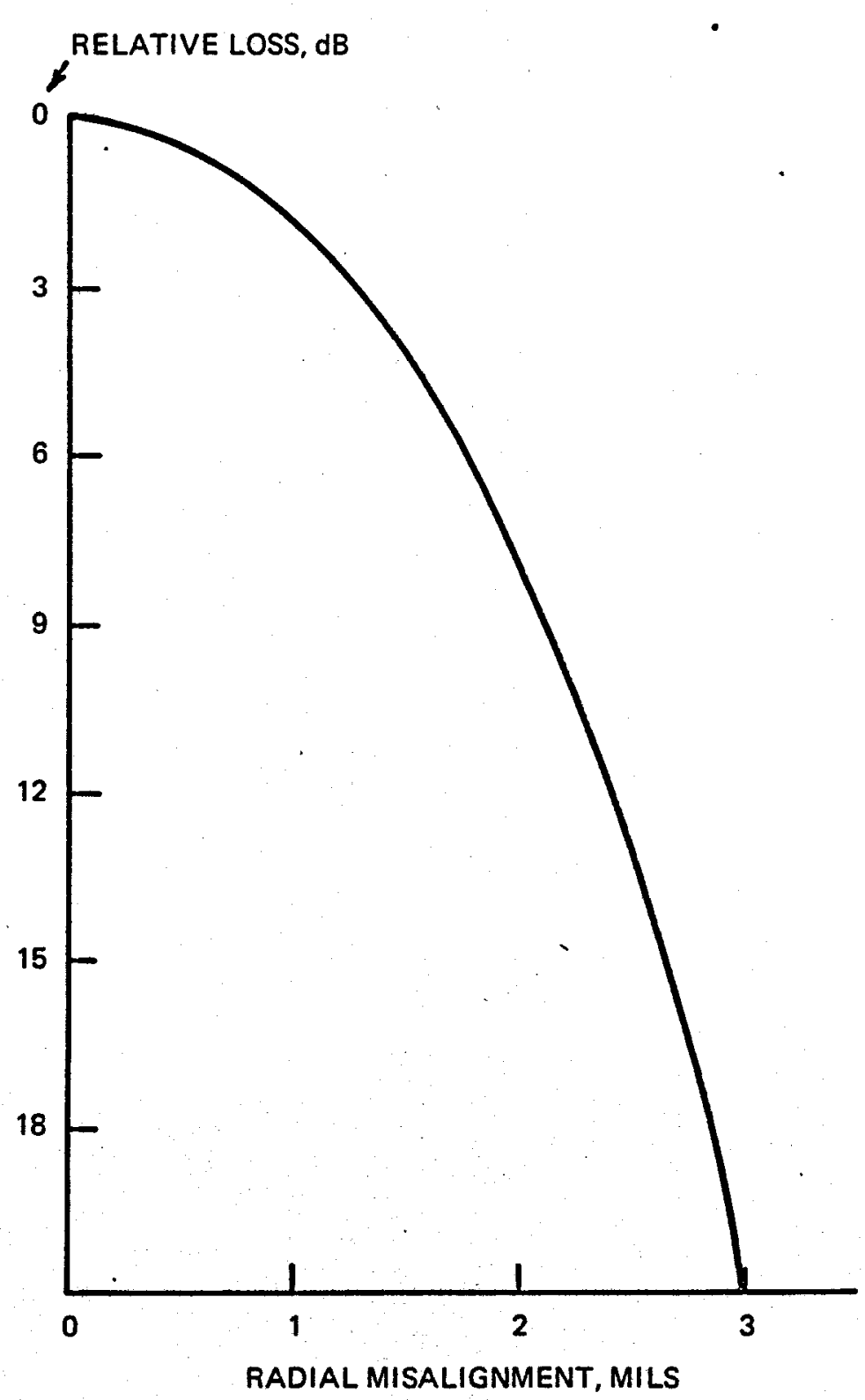

FIGURE 37. EFFECT OF AXIAL MISALIGNMENT ON COUPLING LOSS BETWEEN TWO BUTT-SPLICED 5-MIL DIAMETER FIBERS 


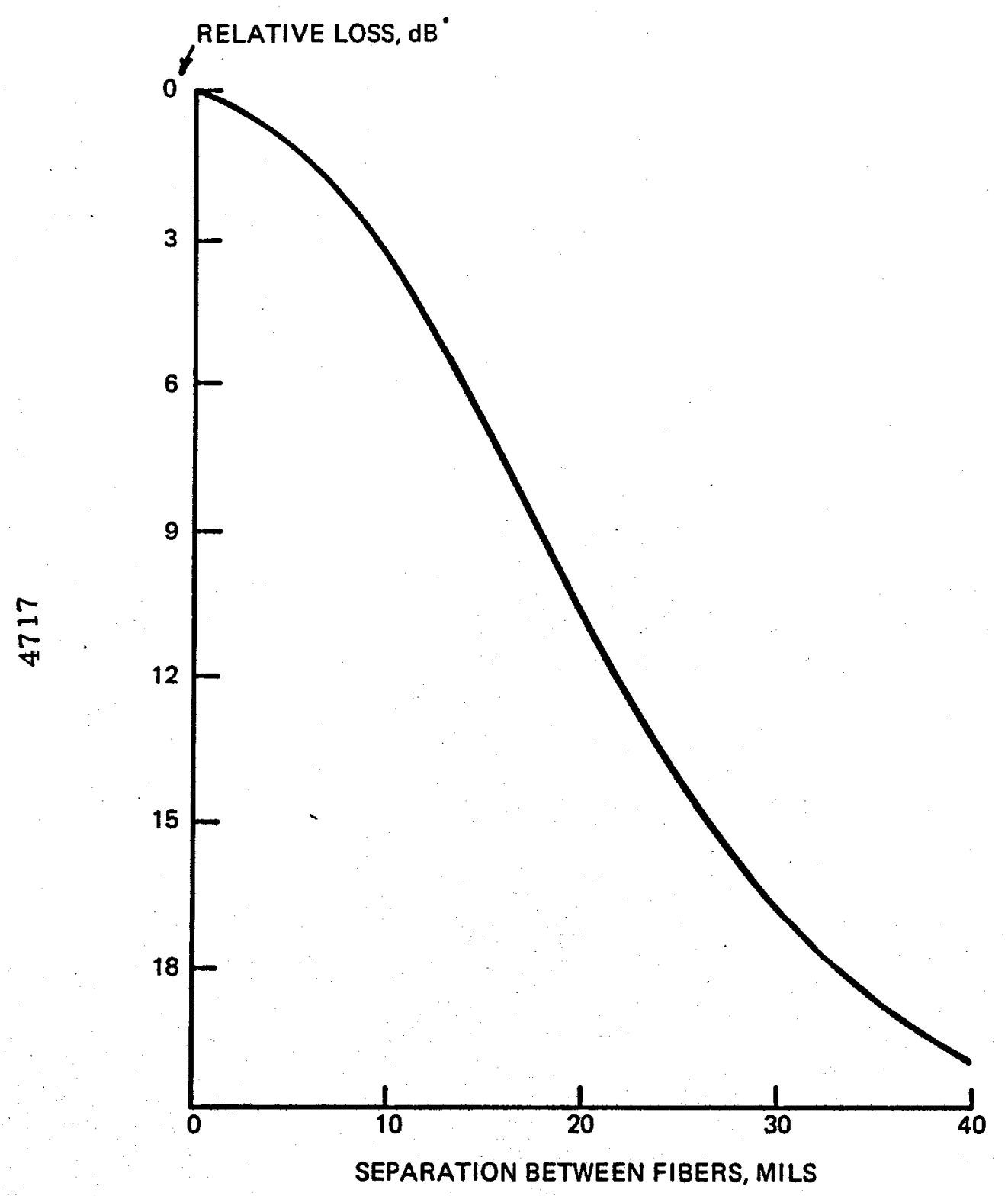

FIGURE 38. EFFECT OF END SEPARATION ON COUPLING LOSS BETWEEN TWO AXIALLY ALIGNED 5-MIL DIAMETER FIBERS 
be attributed to that mechanism. At present, therefore, the data indicate that lateral misalignment is probably a major contributor.

It should also be noted that there are other loss mechanisms that have not yet been analyzed. While limited experimental data on errors in butt splices of fibers in air are available, no data exists for the actual case, in which the system is surrounded by silicone resin. Also, other geometrical errors are possible in the more complex glass-GaAs-glass interface in the prototype compared to the fiber splice.

\section{Results of Temperature Sensor Investigation}

As a result of the work described here, a prototype sensor has been constructed, and two techniques investigated for measuring temperature by means of the dependence of the fundamental absorption edge of a semiconductor. Either technique is apparently suitable for application to well-logging. The more promising method at this time is the photomultiplier threshold detection method. It is both simpler and more direct, i.e., it involves fewer data processing steps, than the maximum slope approach. A second, more compelling reason is that the threshold approach works as predicted, while tests of the maximum slope method have produced unexpected and as yet unexplained results. Because implementation will present difficult problems with either method, it is advisable to choose the method that is amenable to analysis and can therefore be incorporated with confidence in design predictions.

The next logical step after successful completion of the optical cable, is implementation of a system using the threshold method. This involves, first of all, the specification of component requirements for the complete temperature logging system. Thus, before detailed design can begin one requires data on source intensity needs, sensor details and detector sensitivity requirements. This, in turn, requires a systematic study leading to performance predictions for the system as a whole.

Consider, for example, the problem of specifying the source intensity. From the results reported here, we already know that the source should be as intense as possible. However, we do not yet have sufficient data to determine the minimum acceptable source intensity, nor can we estimate how much the performance will improve by employing brighter sources. These are questions which must be answered before the source strength, or even the type of source, can be specified with confidence.

There are also uncertainties regarding the sensor element itself. All experiments performed to date have involved either gallium arsenide or indium phosphide, which were selected largely because they are readily available in convenient form. It is possible, however, that some other material may offer advantages. For example, cadmium telluride may prove to be better because its absorption edge occurs at shorter wavelengths than GaAs, and all available detectors, including photomultiplier tubes, are more sensitive at shorter wavelengths. Before the final system configuration is established, performance data should be examined for complete systems employing other sensor materials. Crystal thickness is also a parameter requiring optimization.

\section{B. Thermal Neutron Detector}

\section{Neutron Logging}

One of the logging techniques expected to be of great usefulness in the evaluation of geothermal reservoirs, is the "neutron log". Neutron logging depends upon bombardment of the rock formations with high-energy neutrons and determining, by one means or another, the rate at which the neutrons are thermalized. Under properconditions, the result shows significant information about the rock matrix, in particular, the rock porosity.

A typical technique for neutron logging is the introduction of a source of fast neutrons, such as 
Californium $\left(\mathrm{Cf}^{252}\right)$, together with a thermal neutron detector, in a fixed physical configuration in the bore hole. With the proper choice of source-detector spacing, the thermal neutron flux at the detector will be a function of the thermalization rate, which is in turn strongly dependent upon the water content of the rock. A low flux of thermal neutrons, for example, can signify low porosity (low water, or hydrogen ion, content), since many neutrons will escape into the rock before thermalization.

In view of the fact that the neutron log is sensitive to parameters which may reflect formation porosity, the development of high-temperature neutron logging equipment should be assigned a high priority in the list of geothermal exploration tools. If an optical transducer could be developed, sensitive to one of the several measurement parameters from which a neutron log can be derived, it would form a highly useful complement to the optical temperature log. Study of such a sensor thus is a natural component of the optical logging investigation.

\section{Preliminary Studies}

As was the case in the development of a temperature sensor, the first step in the study of neutron counting methods was the selection of an approach. Initially, two concepts emerged. One was the scintillation approach, in which a neutron, through its interaction with the sensor element, produces a pulse of infrared radiation which is transmitted via an optical fiber to a counter at the surface. The other idea was dependent on color center production, and would base a measurement of neutron flux on the steady state transmission of a radiation-sensitive material. The latter approach was dismissed because it was found that color center production rates for thermal neutron interactions are much too small to be useful in the low flux fields of interest here.

Early attempts to find a suitable scintillator material were focused on crystals normally used in or considered for thermoluminescent dosimetry. The requirement that the wavelength of the luminescence be suitable for fiber optic transmission eliminated most such materials. from further consideration. Those that were not eliminated, and still show some promise, include $\mathrm{CaS}, \mathrm{CaSO}_{4}: \mathrm{Sm}$ and $\mathrm{Li}_{2} \mathrm{~B}_{4} \mathrm{O}_{7}$. The latter compound is the most attractive, because both $L i$ and $B$ have very large cross sections for thermal neutron capture with resultant alpha emission. One would expect that as the alpha particles lose energy through ionization processes in the material, luminescence would occur at wavelengths characteristic of the material (in the red and near infrared). However, no reports of this expected scintillation effect were found in the literature.

Continuing literature review eventually revealed that a room temperature, visible light scintillator with high efficiency for thermal neutron detection was designed and fabricated some twenty years ago. ${ }^{(19)}$ This scintillator made use of a boron compound and a phosphor. Boron, through the ${ }^{10} \mathrm{~B}(\mathrm{n}, \mathrm{\alpha})$ reaction, gives rise to a flux of high energy alpha particles, this flux being proportional to the incident thermal neutron flux. Ionization created by the alpha particles in the phosphor then produces luminous pulses at wavelengths characteristic of the phosphor. Thus, at this point, effort was concentrated on finding a suitable phosphor or other luminescent device for operation in the infrared at high temperature.

\section{Cadmium Sulphide Scintillator}

Further review of the literature on scintillator materials showed that cadmium sulphide (CdS), doped with copper and chlorine, emits radiation in two broad bands centered at $820 \mathrm{~nm}$ and $1020 \mathrm{~nm}$ when subjected to ionizing radiation at room temperature. ${ }^{(20)}$ Although either band would be suitable from a fiber optic transmission standpoint, it is expected that only the longer wavelength band will be useful at high temperatures. This is because experiments with a similar material $(\mathrm{ZnS})$ show that the luminescent efficiency of the shorter wavelength emission drops off rapidly as the temperature is increased above about $100^{\circ} \mathrm{C}$, while the longer wavelength. emission persists at temperatures up to about $300^{\circ} \mathrm{C}{ }^{(21)}$ 
However, from data on the temperature dependence of the energy band gap in CdS it is expected that at temperatures in the $200^{\circ} \mathrm{C}$ to $300^{\circ} \mathrm{C}$ range, the band centered at $1020 \mathrm{~nm}$ at room temperature will shift to $1200 \mathrm{~nm}$ to $1300 \mathrm{~nm}$, which is slightly too long for efficient transmission in presently available optical fibers. On the other hand, experiments with alloys of $\mathrm{CdS}$ with $\mathrm{ZnS}{ }^{(22)}$ show that the addition of $\mathrm{Zn}$ shifts the emission to shorter wavelengths, thus suggesting that alloying with $\mathrm{ZnS}$ would be helpful. From such experimental data it was estimated that an alloy containing about $85 \% \mathrm{CdS}$ by weight and $15 \% \mathrm{ZnS}$ by weight should emit radiation in the $1000 \mathrm{~nm}$ to $1100 \mathrm{~nm}$ optical fiber transmission window at $200^{\circ} \mathrm{C}$ to $300^{\circ} \mathrm{C}$. Thus, the phosphor we proposed to use is a CdS-ZnS alloy doped with copper and chlorine.

In choosing a boron compound to mix with the phosphor, one needs a material with high melting point, high boron content and minimum optical absorption in the $1000 \mathrm{~nm}$ to $1100 \mathrm{~nm}$ band. Although boron nitride is not ideally transparent at these wavelengths it satisfies the other requirements well, and is readily available in the form of a fine powder. It was therefore expected that a mixture of the CdS-ZnS phosphor with boron nitride in a high temperature plastic would serve as an efficient, high temperature thermal neutron scintillator.

A mixed CdS-ZnS phosphor with a $\mathrm{Cd}$ to $\mathrm{Zn}$ ratio of $4: 1$ and containing $2.5 \times 10^{19} \mathrm{Cu}$ atoms $/ \mathrm{cm}^{3}$ and $2.5 \times 10^{20} \mathrm{Cl}$ atoms $/ \mathrm{cm}^{3}$ was prepared according to our specifications by Cleveland Crystals. Preliminary experiments with this material were not promising. When subjected to alpha particle irradiation from a weak Americium source, no scintillations could be observed. During preparations for further experiments, a second detector concept was developed, and results of its evaluation were sufficiently successful to recommend it over the CdS-ZnS approach. Further work on the CdS scintillator was therefore abandoned.

\section{Zinc Sulphide-YAG Scintillator}

a. Analysis. This approach also makes use of the short wavelength emission from an efficient boron-phosphor mixture, but the resulting radiation (visible light) is then absorbed in a third medium. This medium is in the form of a crystal in which are created short-lived, excited electronic states. The photoexcited crystal then decays by emission of long wavelength radiation suitable for fiber optic transmission.

The solid-state laser crystal yttrium aluminum garnet (YAC), with $\mathrm{Nd}^{3+}$ doping, is nearly ideal for this purpose. As is illustrated in Figure 39 it has strong absorption bands centered at about $\mathbf{5 8 0}$ and $\mathbf{7 4 0}$ $\mathrm{nm}$, and a very intense emission band at $1060 \mathrm{~nm} \cdot{ }^{(23,24)}$ The absorption band at $580 \mathrm{~nm}$ lies near the peak in the room temperature "green" emission spectrum of copper-activated $\mathrm{ZnS}$ which is centered at $523 \mathrm{~nm}$. At higher temperatures the peak in the $\mathrm{ZnS}$ spectrum shifts to longer wavelengths, which should make the match between $\mathrm{ZnS}$ emission and YAG absorption even better.

One way this two-step scintillation principle might be implemented is illustrated in Figure 40 . In this design, $\mathrm{ZnS}$ and $\mathrm{B}^{10}$ are applied as coatings on the outside of the YAC crystal. When slow neutrons strike $B^{10}$ (with a cross section of 2400 barns), 1.47 MEV alpha particles are emitted. These alpha particles strike the fluorescent $\mathrm{ZnS}$ which in turn "pumps" the YAG crystal, which re-emits strongly at the longer wavelengths. In this way a neutron captured in the coating results in a strong pulse of light of suitable wavelength to be transmitted through the fiber optics to a remote detector.

b. Experimental Investigation. One slow-neutron counter normally used in well- logging is the boron trifluoride $\left(\mathrm{BF}_{3}\right)$ detector. Typically, the efficiency of a $\mathrm{BF}_{3}$ counter is about $20 \%$, which means that $20 \%$ of the neutrons entering the sensitive volume of the detector result in counts. Thus, if a YAC detector of comparable size is to perform as well as a $\mathrm{BF}_{3}$ counter, it should have a comparable efficiency. The principal objective of our experimental study of the YAG counter was, therefore, to obtain an estimate of its counting efficiency.

In the preliminary investigation reported here we did not obtain a direct measurement of neutron counting efficiency, which would have required fabrication of a complete system followed by 


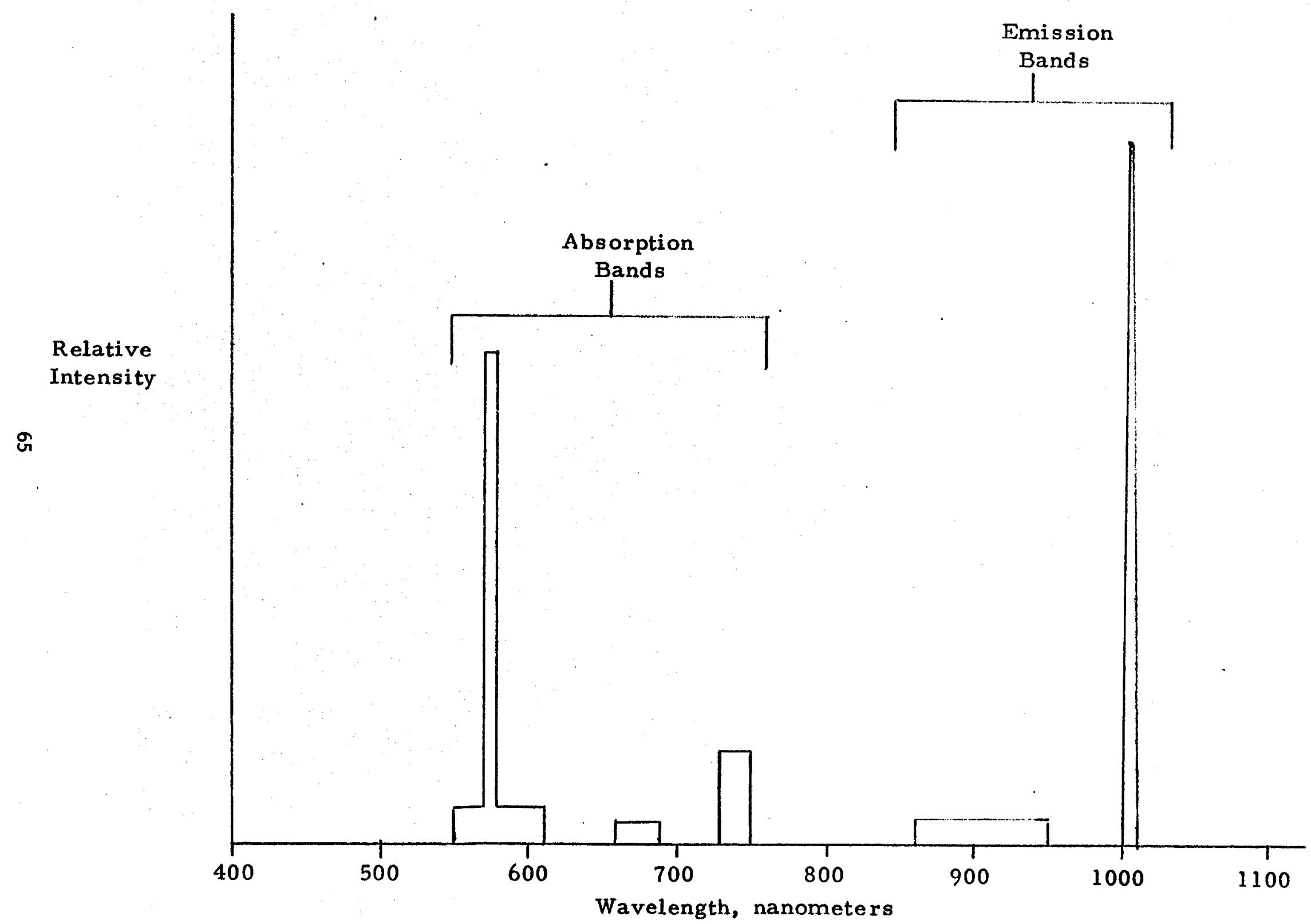

FIGURE 39. PRINCIPAL ABSORPTION AND EMISSION BANDS OF $\mathrm{Nd}^{3+}$ :YAG 
\&

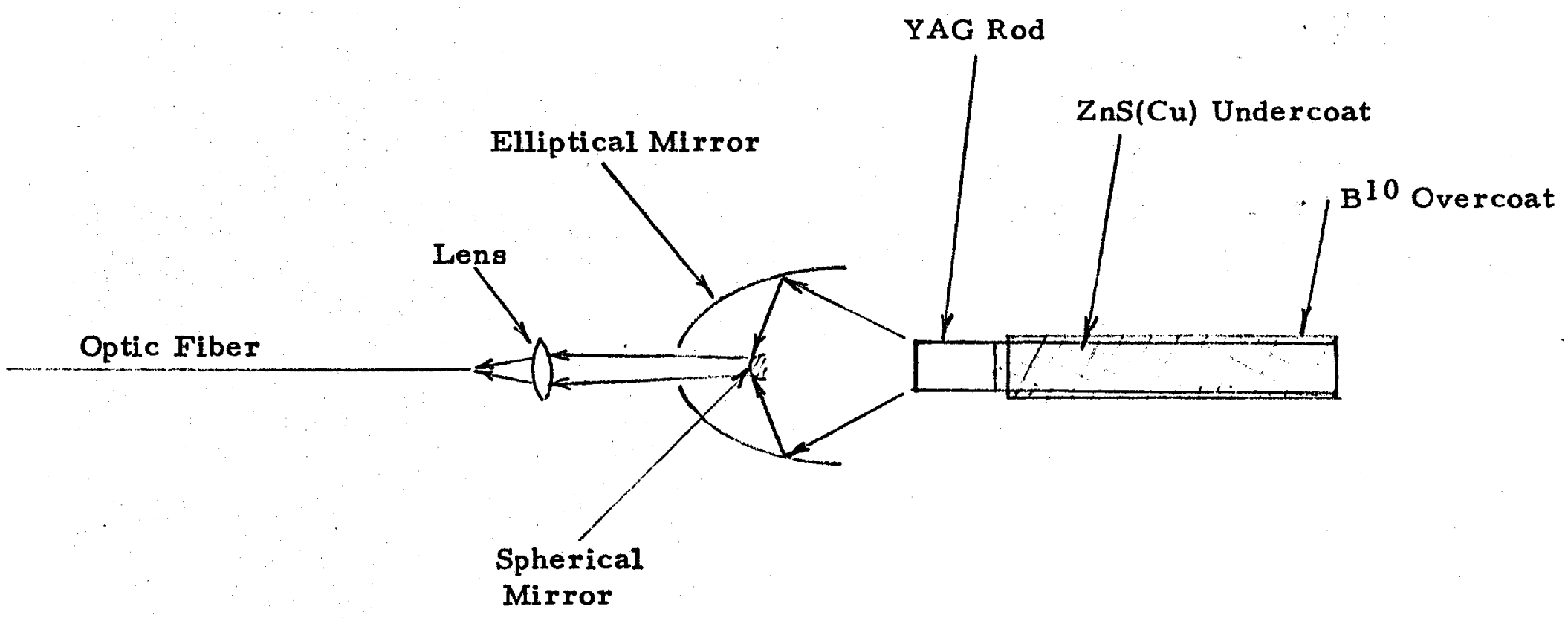

FIGURE 40. SCHEMATIC OF INFRARED NEUTRON SCINTILLATION DETECTOR EQUIPPED WITH SCHWARZCHILD COUPLER TO FIBER OPTIC CABLE. 
calibration tests in a known neutron field. Instead we chose to determine the efficiency for counting alpha particles, which is the principal unknown, and which can be carried out in the optical laboratory. No special radiation shielding precautions are required as with neutron experiments, and the efficiency for converting neutrons to alpha particles by means of the $B^{10}(n, d)$ reaction is easily estimated. The experimental determination of alpha counting efficiency is all that is needed for a preliminary estimate.

Before proceeding with experiments on the ZnS-YAG system, certain questions regarding the properties of the $\mathrm{ZnS}$ phosphor itself required investigation. For example, there was some concern regarding the fact that the luminescent efficiency of $\mathrm{ZnS}$ is known to decrease with increasing temperature. To determine whether this would cause a significant change in counting efficiency, the spectral luminescence of $\mathrm{ZnS}$ at room temperature and at an elevated temperature was measured.

In this experiment a thin $\mathrm{ZnS}$ sample was prepared on a microscope slide and excited with an ultraviolet lamp. The resulting fluorescence was collimated by a lens into a monochromator, and a silicon detector-amplifier system used to record intensity as a function of wavelength. These results, shown in Figure 41, indicate a slight decrease in luminescent efficiency when the temperature is raised from room temperature to $300^{\circ} \mathrm{C}$, but this is more than compensated by a spectral shift such that the emission spectrum of $\mathrm{ZnS}$ more nearly matches the absorption spectrum of YAC at an elevated temperature. As far as the $\mathrm{ZnS}$-YAG system is concerned, the net result is that an increase in temperature has little effect on overall efficiency.

Some experimentation was required to determine how to prepare the $\mathrm{ZnS}$ coating used in this luminescence experiment and in subsequent experiments. The ZnS crystals, as obtained from U. S. Radium Corporation, ranged in size from about 1 to 10 mils $(0.025$ to $0.25 \mathrm{~mm})$. It was therefore necessary to prepare a finer powder before mixing the coating material. To do this the zinc sulfide was ground with a mortar and pestle and sieved through a 325 mesh screen. This screen size eliminates those crystals which are larger than 1.2 mils $(0.03 \mathrm{~mm})$.

Dow Corning 804 was found to be a suitable high-temperature adhesive for binding the zinc sulfide to the glass slides. It was found that a thin film $(0.2 \mathrm{mil})$ of silicone could be uniformly deposited by dipping a slide in the material and using a motor driver system to withdraw the slide at a rate of 3 inches per minute. The specimen is then placed in a preheated oven set at $120^{\circ} \mathrm{F}$ for two minutes. This preheating makes the silicone tacky but still not dry. ZnS powder is then dusted uniformly over the slide. Excess material is removed by tapping the slide, which is returned to the oven and allowed to bake for 2 hours. The silicone tends to flow up and around the $\mathrm{ZnS}$, making a good bond between the $\mathrm{ZnS}$ and the glass. In the final step, acetone is used to remove material from the opposite side of the slide.

The thickness of the $\mathrm{ZnS}$ film can be a critical parameter because the material is not transparent to green light. However, if the film thickness does not greatly exceed the range of alpha particles in $\mathrm{ZnS}$ (which is quite small), self-absorption of the light in the coating is low. It has been reported that the optimum thickness for alpha particle detection is 0.02 to $0.05 \mathrm{~mm} .^{(25)}$

To verify this result, and to determine how sensitive light output is to film thickness, specimens were prepared with coatings of different thickness, by varying the rate of withdrawal from the liquid silicone. These specimens were then placed next to an Americium ( $\left(\mathrm{m}^{241}\right)$ alpha source, and scintillation rates recorded using a photomultiplier counting system. The results are tabulated below:

\begin{tabular}{cc} 
ZnS Thickness $(\mathrm{mm})$ & Counts/min. \\
\hline 0.02 & 1931 \\
0.039 & 1954 \\
0.05 & 2053
\end{tabular}


1

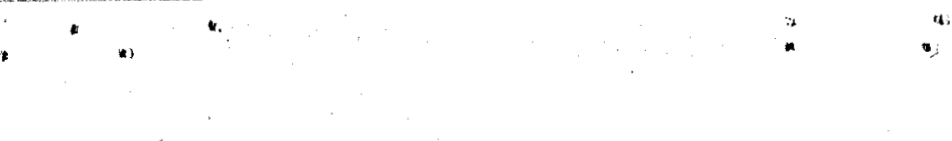

$\infty$

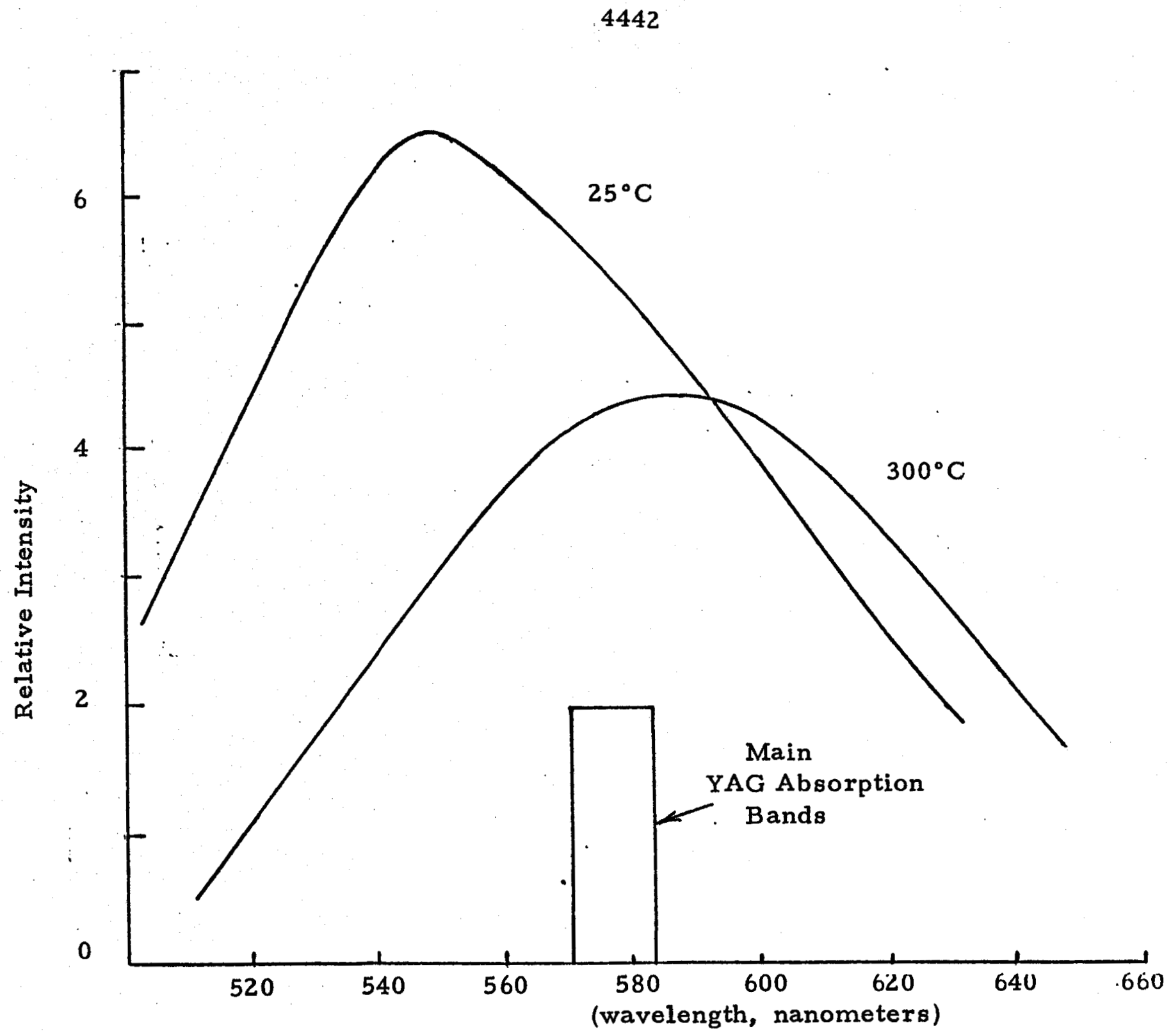

FIGURE 41. RELATIVE LIGHT OUTPUT OF ZnS (Cu) PHOSPHOR WITH TEMPERATURE SHOWING POSITION OF MAIN YAG ABSORPTION. 
Since there is no significant difference in these readings with the Americium source, film thickness in the range of 0.02 to 0.05 is adequate. However, the alpha particle energy in this case is $5.48 \mathrm{MeV}$, while the energy of the particle emitted in the $B^{10}(n, x)$ reaction is only $1.47 \mathrm{MeV}$. Since the range of an alpha particle is proportional to its energy, one would expect the optimum thickness for alpha particles from $B^{10}$ capture to be at the lower end of this range.

To determine the alpha counting efficiency of the ZnS coated YAC crystal, it was first necessary to determine the alpha particle flux incident on the crystal. This was done by covering the face of a photomultiplier tube except for an opening of $2 \times 20 \mathrm{~mm}$, the approximate cross sectional area of the YAC crystal. A ZnS coated slide was then placed between an alpha source (in this case a uranium source was used) and the masked photomultiplier. Because almost all of the alpha particles striking the $\mathrm{ZnS}$ phosphor give rise to light pulses, the count rate observed with this setup, which was 600 counts/min., is directly proportional to the alpha particle flux passing through a $2 \times 20 \mathrm{~mm}$ slit. In other words, a count rate of 600 counts $/ \mathrm{min}$. corresponds to $100 \%$ efficiency for a detector the size of the YAG crystal.

Next, after coating the YAC crystal with $\mathrm{ZnS}$, it was introduced into the system, together with an infrared sensitive photomultiplier (Type 31004) and a filter used to block the visible radiation from the $\mathrm{ZnS}$, since it is also phosphorescent. The entire assembly, i.e., the alpha source, coated YAG and photomultiplier, was placed in a chamber at $-30^{\circ} \mathrm{C}$ to reduce dark current noise from the photomultiplier. Figure $\mathbf{4 2}$ shows typical signals resulting from the YAC scintillator before and after pulse shaping.

The count rate observed with this arrangement was 60 counts/min. However, because the filter blocked some of the $1060 \mathrm{~nm}$ radiation from the YAC crystal, this is not representative of the actual efficiency of the ZnS-YAG scintillator. To determine the loss caused by the filter, we measured the filter attenuation at $1060 \mathrm{~nm}$ in a separate experiment. It was found that the attenuation factor is 0.45 , which means that the actual light from the YAC was roughly double that observed through the filter. If we assume that doubling the intensity causes the count rate to double, then the corrected YAC count rate is 120 counts/min. Thus, our estimate of the efficiency for alpha particle counting is $20 \%$.

To convert this figure to an expected neutron counting efficiency we can assume that, because the capture cross section of $\mathrm{B}^{10}$ is extremely large, all thermal neutrons incident on the outer layer of boron are captured and produce $1.47 \mathrm{MeV}$ alpha particles. However, the directions of these alpha par- ticles are randomly distributed so that only about half reach the $\mathrm{ZnS}$ layer. If $20 \%$ of the $\mathrm{ZnS}$ scintillations lead to YAC pulses, as our experiment indicates, then the estimated neutron counting efficiency is about $10 \%$.

Although this is about half the efficiency of a typical $\mathrm{BF}_{3}$ counter, it is still large enough, since $\mathrm{BF}_{3}$ is affected by volume, while the $\mathrm{B}^{10} \mathrm{YAC}$ efficiency is a function of total effective area. The slow neutron flux one must measure in a well-logging application is of the order of 100 neutrons $/ \mathrm{cm}^{2}$-sec, with $10 \%$ efficiency, a YAG scintillator with $10 \mathrm{~cm}^{2}$ surface area would produce a count rate of 100 counts $/ \mathrm{sec}$. This is roughly equivalent to a coated YAC rod $0.5-\mathrm{cm}$-diameter by $6-\mathrm{cm}$-long, one of modest size.

\section{Coupler Design}

In considering methods for collecting the light from the thermal neutron detector described in the preceding paragraphs and coupling it to a single fiber, a coupler design concept was developed that may also have other important applications. In particular, this design offers promise as a means of efficiently coupling light from a relatively large source, such as a tungsten filament, into a single fiber.

The coupler design, known as the Schwarzchild system, is based on work by Burch ${ }^{(26,27)}$ and others who have designed two-mirror microscope objectives. These objectives collect the light emitted by a source and project it in a narrow, almost parallel beam. The basic concept is to place the source at one 


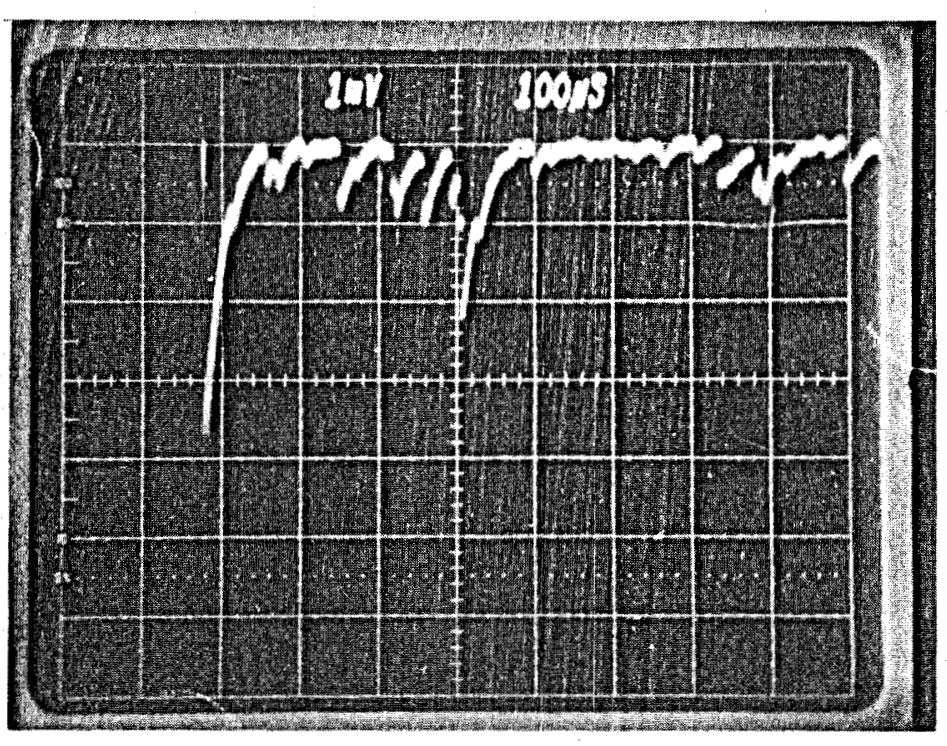

a

$\stackrel{-1}{m}$

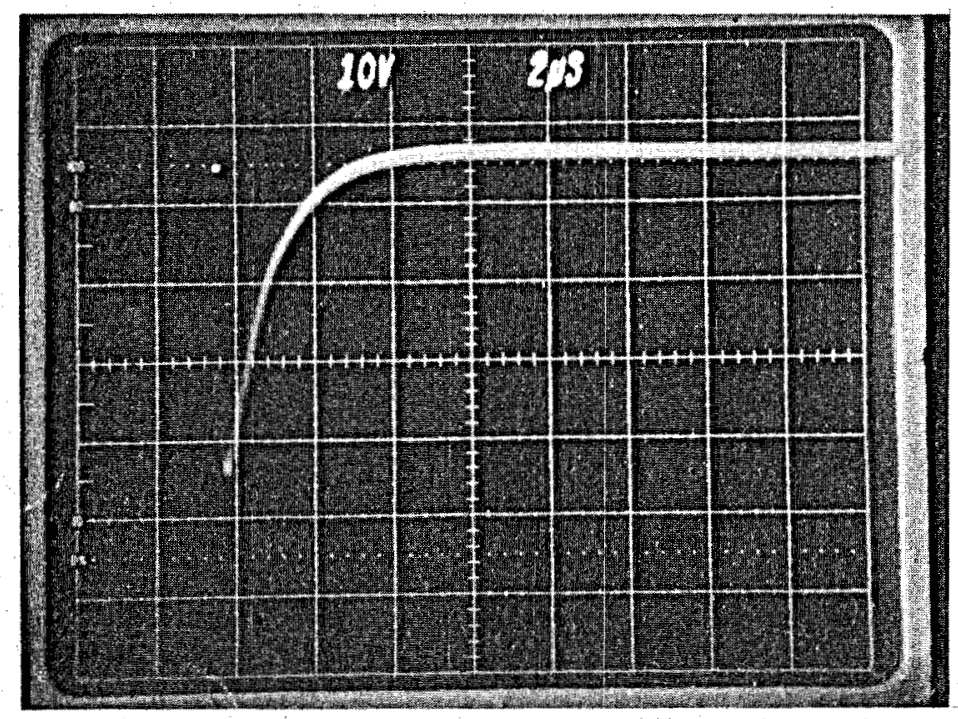

b

FIGURE 42 PHOTOMULTIPLIER SIGNALS FROM ZnS-COATED YAG CRYSTAL UNDER ALPHA-PAR TICLE BOMBARDMENT. S1 response (a) raw anode signal, (b) typical pulse after shaping by charge amplifier. 
focal point, " $\mathrm{a}$ " in Figure 43, of an elliptical mirror, and a'small spherical mirror near the second focal point, " $b$ " of the ellipse. The positions are adjusted to give a nearly parallel beam of light through the opening, " $c$ ", in the large mirror. The lens, " $d$ ", is used to focus the beam on the end of a fiber. A schematic of the complete detector- coupler combination was shown in Figure 38.

The computer program OPDEAN, discussed more fully in Appendix B, was used to investigate the parameters of interest in the coupler design. Figure 43 shows a satisfactory configuration for the case in which light rays striking the large mirror from within a half-cone angle of $58^{\circ}$ will exit through the small hole at " $c$ ". The ray-trace computer program was used to determine the fraction of rays leaving the object that would strike both mirrors and pass through " $c$ ". Several ray-trace simulations, each with a different small mirror position, were run using 150 rays in each problem. The configuration shown in Figure 43 produced the largest fraction of rays that leave the source within a $58^{\circ}$ cone and pass through the aperture to the focusing lens. In this design, the radius of the small mirror is 2 units, the radius of curvature of the ellipse is 13.1 units, its eccentricity is 0.11 , and the width of the opening " $c$ ", is 2 units.

The computer program was also used to determine the sensitivity of coupling efficiency to the physical dimensions of the source. Results of these calculations are summarized in the contour map shown in Figure 44. This contour map summarizes the effect of a point light source located in an area near " $a$ " in Figure 43, but removed from it. For this particular configuration, the total source angle encompassed is $58^{\circ}$ each side of the axis. The contour plot shows, for example, that for points along a vertical line at least $0.1-\mathrm{cm}$-long, located $2.5-\mathrm{cm}$ behind the rear surface of the spherical mirror (slightly in front of "a"), about $50 \%$ of the light emitted within the $58^{\circ}$ acceptance angle will be contained in the output beam. This means that light from a relatively large source, for example the YAC crystal, could be coupled into a fiber with high efficiency (the model considers only geometric losses and not those incurred by mirror imperfections, etc.).

A laboratory experiment was designed to verify the system, by measuring the coupling improvement between a fiber and a tungsten lamp. This data will be valuable in designing a system for coupling the neutron scintillation detector to the cable. In Figure 45 is shown a photograph of the laboratory setup with the two mirrors collecting the light, and a microscope objective as a focusing element. This lamp was used in much of the experimentation done during the project. Ordinarily, coupling is accomplished by placing the prepared fiber end as close to the lamp envelope as possible, directly in line with the filament. By careful position control, the optimum physical position can be located. This has been found the most efficient way to couple to a tungsten lamp, without reflection optics. Even so, the coupling efficiency is very low. With the arrangement illustrated in Figure 45, coupling was improved under the same conditions, a total of $13 \mathrm{~dB}$ with the Schwarzchild system, even though the acceptance angle is still very small due to the small mirror used. The concave mirror used here was spherical rather than elliptical, and was only $5-\mathrm{cm}$ in diameter. The smaller mirror was $6-\mathrm{mm}$ in diameter and had a $9 \mathrm{~mm}$ radius of curvature. Because both mirrors were spherical, and their dimensions were far from optimum, much better results can be expected from a properly designed system.

\section{Results of Neutron Sensor Investigations}

Experimental studies of the alpha particle counting efficiency of the YAG scintillator show that the idea is highly promising. While preliminary estimates indicate that the thermal neutron efficiency is somewhat lower than that of $\mathrm{ABF}_{3}$ counter, the detector size required to produce comparable count rates under similar flux conditions is reasonable. Thus, at this point, the $B^{10}-\mathrm{ZnS}$ coated YAC crystal appears to have good feasibility as an efficient scintillator for thermal neutron well-logging. 


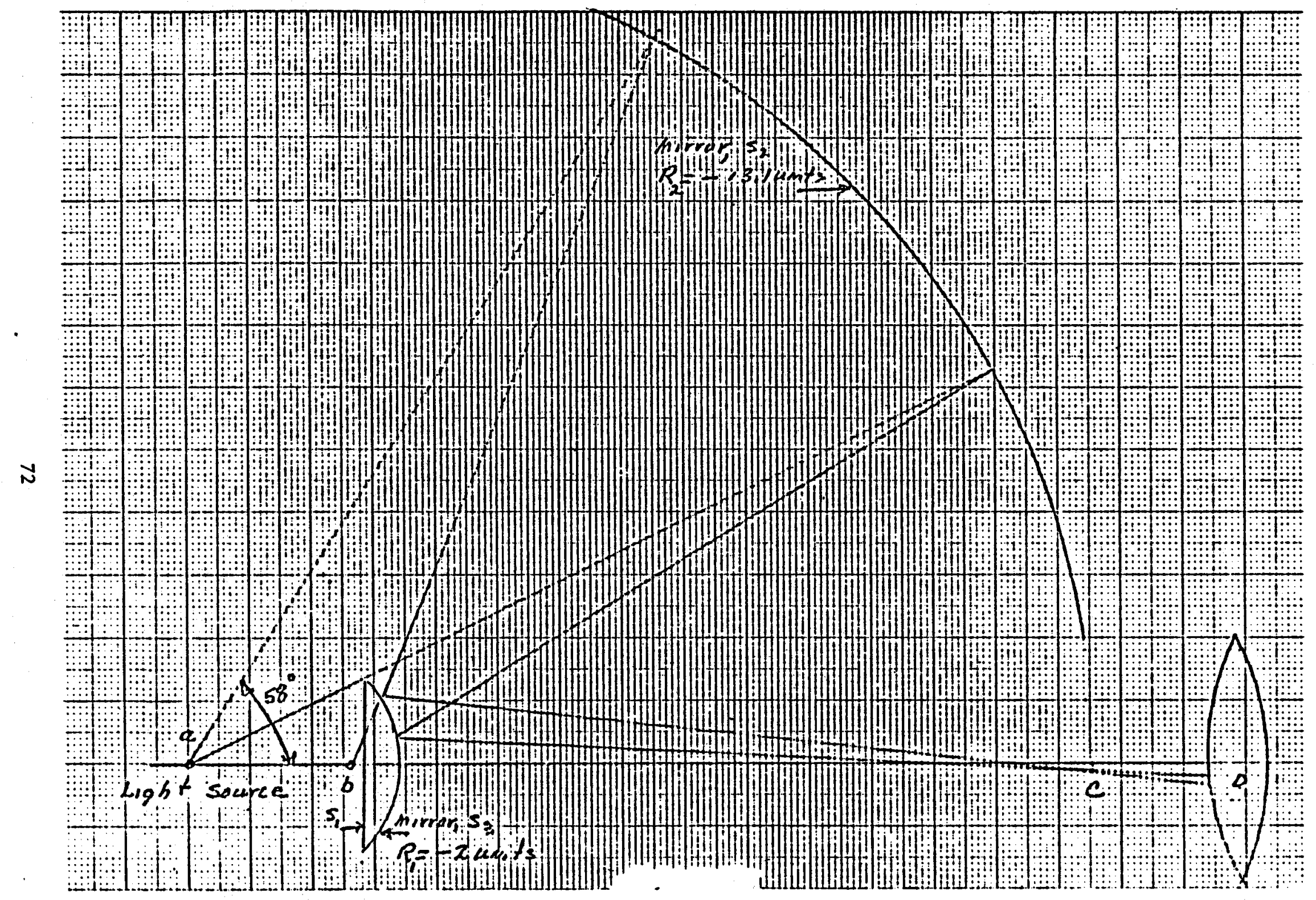

FIGURE 43. SCHEMA TIC OF TWO-MIRROR OPTICAL COUPLER. Source is near "a", collected beam exits through small hole in eliptical mirror at "c". 


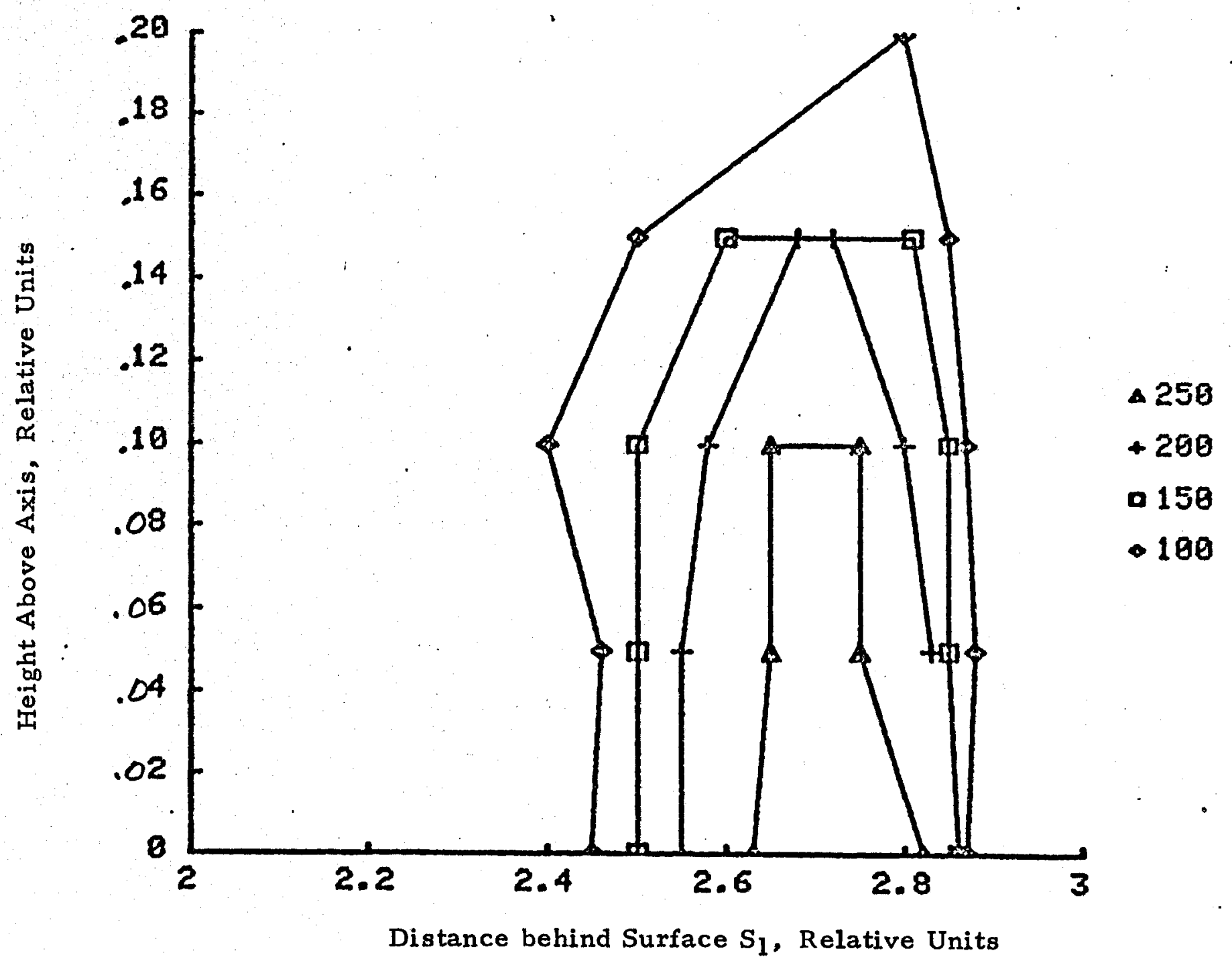

FIGURE 44. CONTOUR PLOT OF LIGHT RAYS EXITING FROM COUPLER IN FIGURE 43 FOR 300 RAYS EMITTED FROM POINTS DISPLACED VERTICALLY AND HORIZONTALLY FROM IDEAL SOURCE POSITION AT "a". 


\section{C}

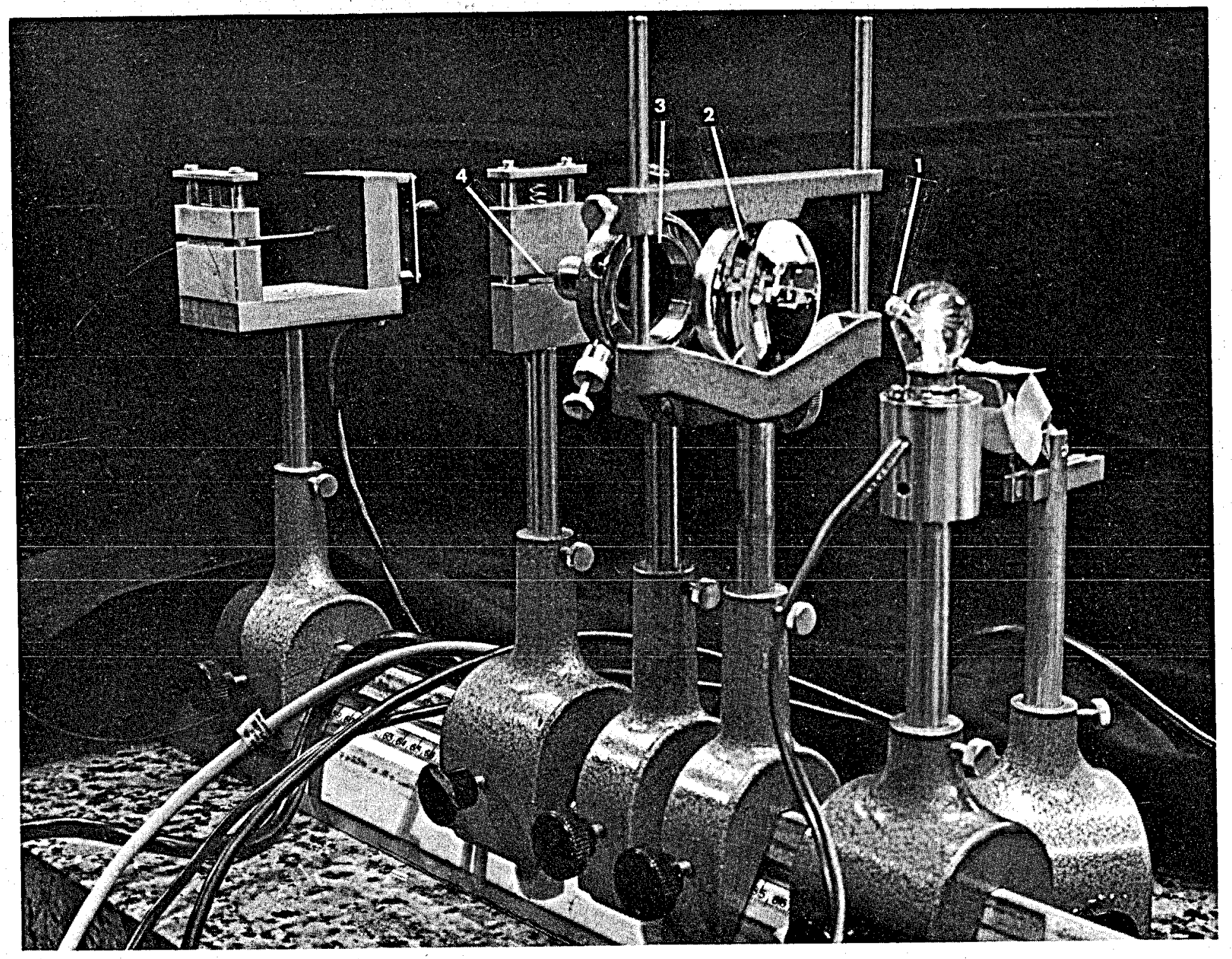

FIGURE 45. EXPERIMENTAL ARRANGEMENT FOR INVESTIGATING SCHW AR ZCHILD REFLECTION SYSTEM FOR COUPLING EXTENDED SOURCE TO OPTIC FIBER; 1) SMALL SPHERICAL MIRROR, 2) ELLIPTICAL MIRROR, 3) FOCUSSING LENS, 4) OPTIC FIBER. 
The two-mirror Schwarzchild method for coupling the radiation from the YAG crystal to an optical fiber also holds promise. Ray-trace calculations and preliminary experimental data indicate that this method offers substantial improvement over conventional coupling techniques, particularly when applied to large, divergent sources of radiation such as the YAC scintillator.

\section{Electro-Optic Systems}

One of the useful applications of the optical logging system is expected to be the transmission of signals from temperature-hardened electronic packages. This would eliminate many high temperature cable problems, and simplify high data-rate communication. It requires a down-hole optical source or optical modulator capable of interfacing electrical to optical systems, and transmitting data to the surface. In this Section are discussed the use of light emitting diodes, various types of modulators, and photoelectric cells for the down-hole generation of electrical power.

\section{Light Emitting Diodes}

A straightforward approach to optical signal transmission is an optical source down-hole, modulated by the well-logging signal. Such a system is potentially capable of interfacing any electrical signal to an optical transmission line. A review of the literature indicates that one of the best prospects for this role is a GaAs PN junction with a high silicon doping level. For evaluation of the concept, a GaAs LED with approximately the proper silicon doping level was obtained. The diode was tested in a high-temperature oven with its optical output monitored by an outside detector and monochromator through an optical fiber. Temperature of the diode was controlled by the oven, and current supplied to the diode only for periodic brief intervals. This allowed determination of the actual output of the diode as a function of temperature with insignificant effect from internal power dissipation.

The results of these temperature tests are shown in Figure 46. Two effects can be noted as the temperature increases: first, the output declines to approximately $20 \%$ of the room temperature value at $200^{\circ} \mathrm{C}$; second, the peak-output wavelength of the emitted light increases. At $210^{\circ} \mathrm{C}$ the melting point of the solder connecting the internal leads in the diode was reached.

From the literature, it is evident that a properly designed element, which included solderless construction, could be operated to temperatures above $300^{\circ} \mathrm{C}$ with useful output. This is shown by projecting the intensity data from the test diode, presented in Figure 47. A silicon-doped GaAs diode can doubtless be constructed to handle the data transmission chore in a hybrid system. Further details on the experiment and on the prospects for improving the high temperature performance of LED's are reported in Appendix C.

\section{Modulators}

Modulators to be considered for use down-hole can be classified in six major categories: (1) electromechanical, (2) electro-optical, (3) magneto-optical, (4) elasto-optical, (5) semiconductor, and (6) miscellaneous. General requirements for down-hole modulators are (1) low driving power, (2) low driving voltage, (3) operation at elevated temperatures, (4) good depth of modulation, and (5) low insertion loss.

Before discussing modulator types it is desirable to review these requirements against which each type will be evaluated. The most important factor to be considered is the environment in which the modulator will operate. The assumption is made that environmental protection for the modulator can be provided by appropriate packaging. Consequently, the modulator will have to survive temperatures up to $300^{\circ} \mathrm{C}$, but not direct contact with down-hole materials. In addition, the modulator should operate with only a few $(-100$ ) milliwatts of electrical power at a low voltage (in the range from 3 to 24 volts) and to provide reasonable modulation depth. It should be capable of coupling with low losses ( $-100 \mathrm{~dB}$ total) to optical fiber transmission lines. Meeting all these conditions simultaneously may not be practical and compromises may be necessary. 


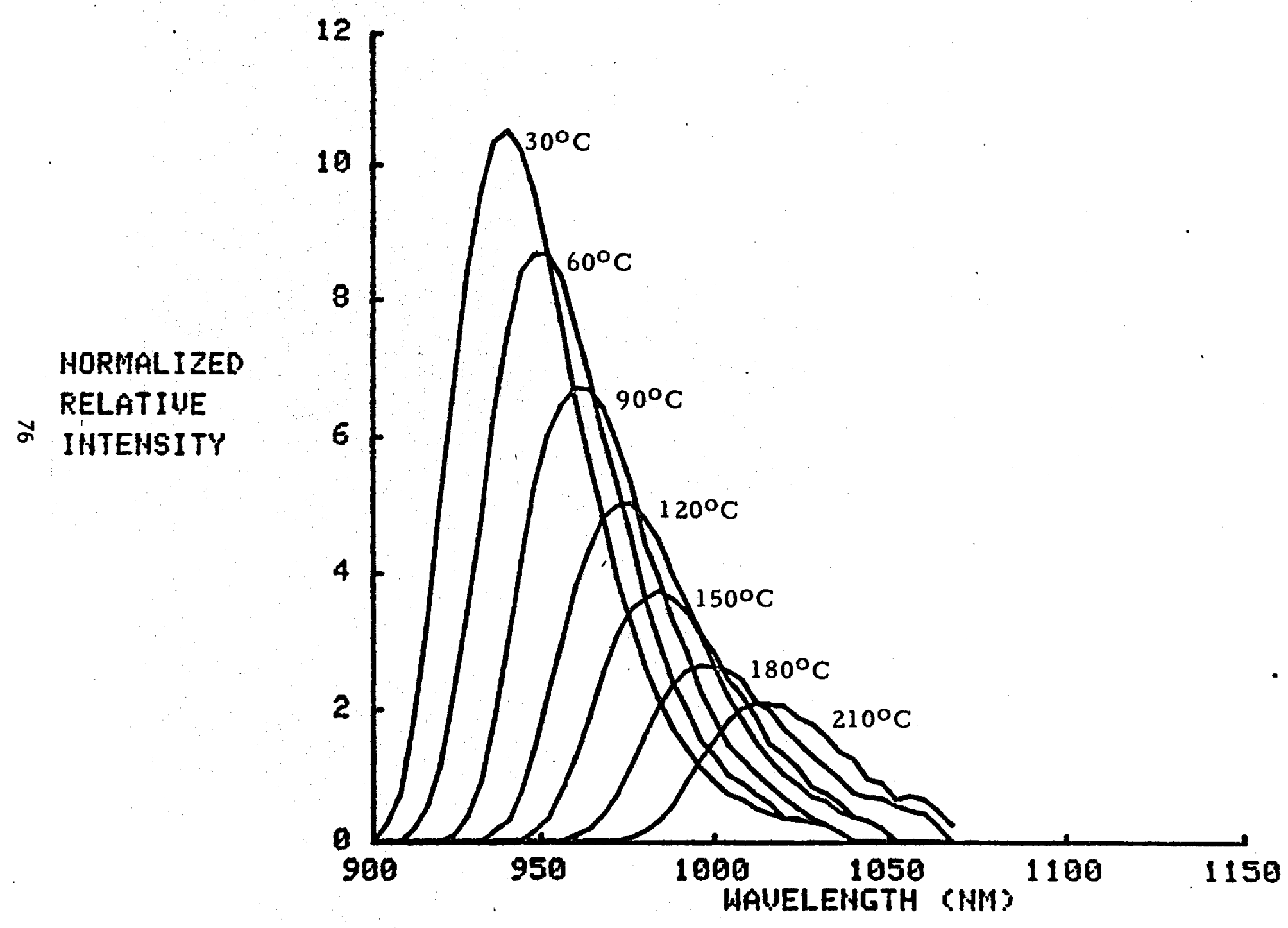

FIGURE 46. NORMALIZED OUTPUT OF GaAs PN LED AS A FUNCTION OF WAVELENGTH AND TEMPERATURE. 
FIG 47: NORMALIZED RELATIUE LIGHT OUTPUT GAAS PN JUNCTION AT ELEUATED TEMP

$\checkmark$ RELATIUE OUTPUT

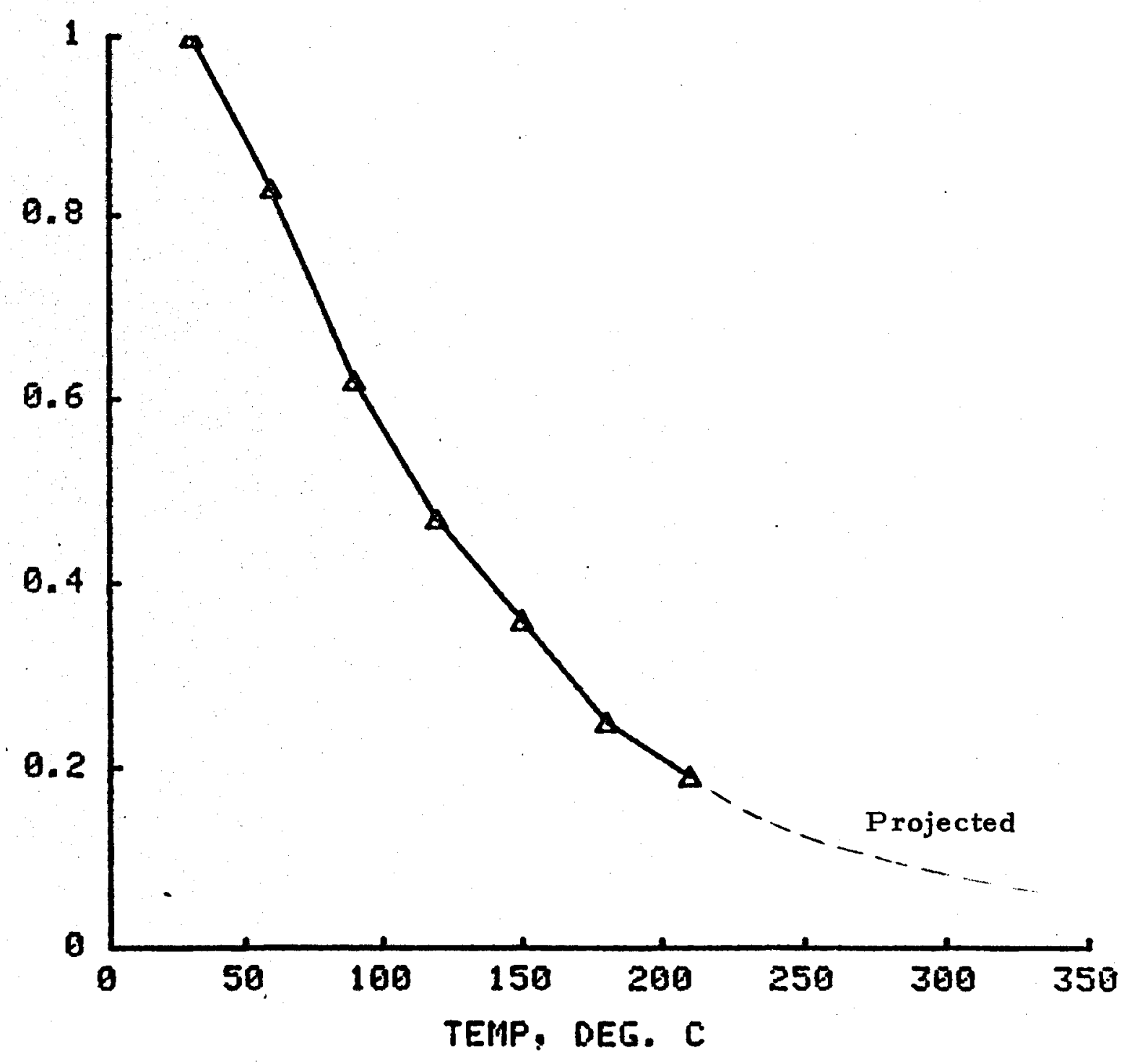


a. Electromechanical Modulators. Several types of electromechanical modulators have been proposed. In general, these devices involve the electrically driven displacement of some optical component such as a mirror, prism, shutter or aperture wheel. As an example of such devices, we will describe only one concept, namely, modulation by frustrated total internal reflection.

Frustrated total internal reflection is accomplished by modifying the internal reflection properties of a totally internally reflecting corner cube as illustrated in Figure 48 . In (a) the angle of incidence of each ray on the back surface of the cube is greater than $\theta_{c^{\prime}}$, the critical angle, where $\theta_{c}=\arcsin \frac{n_{2}}{n,}$, with $n_{1}$ the index of refraction of the corner cube material and $n_{2}$ that of the surrounding space. In (b) the prism with the same index of refraction as the corner reflector is in contact with the cube and no reflection takes place; i.e., the light passes through the corner cube into the prism and on. In this figure the light passes completely through the prism because it strikes the prism back surface at an angle much less than $\theta_{c}$. In (c) the prism is not touching the corner cube but is closer than one wavelength so that some light is reflected and some passes through, the relative amounts depending on the spacing. If the prism is moved in accordance with the information to be impressed, then the reflected light varies in amplitude in a similar manner, and thus, is modulated.

The chief advantages of this approach are good modulation depth (nearly $100 \%$ ) and low power requirements. While there is, in principle, no temperature effect, the fact that extremely close tolerances must be maintained over a wide range of temperatures, may pose a difficult design problem.

b. Reactive Modulators. Electro-optic, magneto-optic and elasto-optic modulators make use of the change in refractive index of a material induced by the application of electric, magnetic or acoustic (elastic deformation) fields. Reactive modulators can be bulk, waveguide, or surface devices.

The primary electro-optic effects are the Pockels effect and the Kerr effect which are related to the electric field dependence of the refractive index. These effects are normally employed in bulk devices and thus require high voltages to achieve adequate field strength. Optical waveguide applications, which will be discussed later, employ thin film devices that require much lower voltages. For this reason, the waveguide approach is better suited to the present application.

The only magneto-optic effect used for modulation is the Faraday effect, which is magnetic field induced change in the relative refractive index for left and right circularly pólarized light. It is thought that this effect will not prove useful because of relatively high power requirements.

Elasto-optic devices employing acoustic surface waves are attractive because they can be used for frequency and amplitude modulation as well as beam deflection applications. However, because the electrical energy that drives such a device must be converted to acoustical energy in a crystal, insertion losses may lead to higher power requirements than are desirable.

c. Semiconductor Modulators. Semiconductor modulators can be (1) PIN free-carrier, (2) depletion region window, (3) depletion region fundamental absorption, or (4) depletion region polarization modulators. The first is a forward biased device requiring less than $2 \mathrm{~V}$ drive and less than $100 \mathrm{~mW}$ power for modulation depths from $10 \mathrm{~dB}$ to $30 \mathrm{~dB}$ at $20 \mathrm{kHz}$. The other devices are reverse biased and require less power (approaching $1 \mathrm{mV}$ ) but much higher voltages (of the order of $100 \mathrm{~V}$ ). It is thought, therefore, that the free carrier device is better suited to the present purpose.

The PIN free-carrier modulator is an optical absorption device in which the strength of absorption is modulated by controlling the number of carriers injected into the optical path. Efficient operation therefore requires efficient injection which poses a problem at high temperatures. Devices fabricated with wide band gap materials such as $\mathrm{SiC}$ may prove suitable, but have yet to be tested as high temperature modulators. 


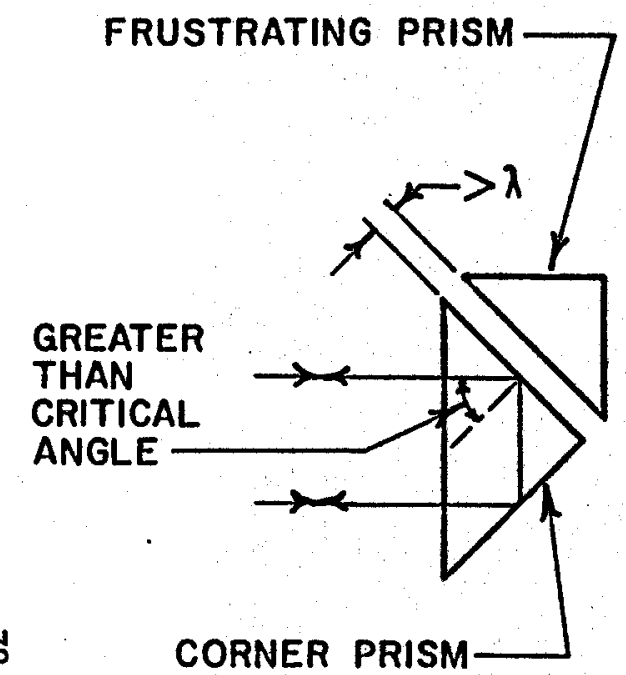

a. Totally reflecting (Prisms separated by a distance greater than one wavelength)

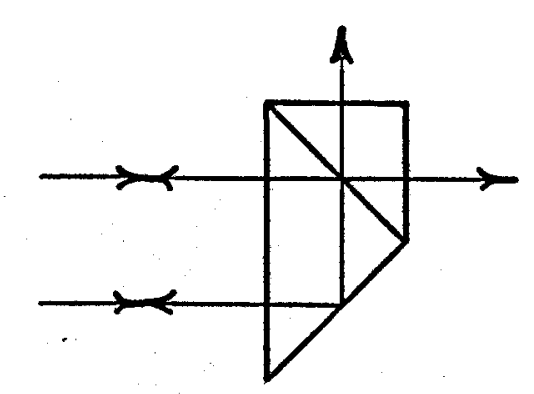

b. Totally frustrated (Prisms in physical contact)

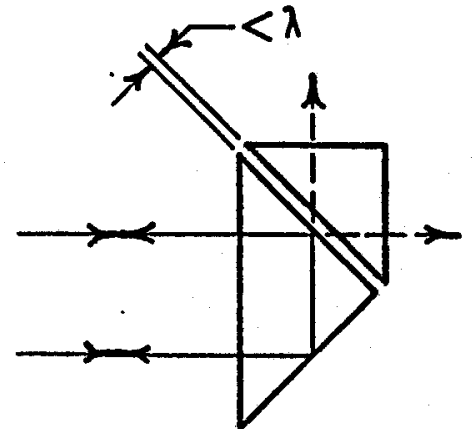

c. Partially frustrated (Prisms separated by a distance less than one wavelength)

FIGURE 48. FRUSTRA TED TOTAL INTERNAL REFLECTION 
d. Waveguide Modulators. Waveguide modulators include hybrid techniques such as variation of evanescent field coupling by varying the effective spacing between two adjacent waveguides. Two parallel electrodes are deposited on an optically polished $X$-cut surface of lithium niobate. The voltage applied between the electrodes produces a high electric field in the crystal in the region just below and between the electrodes. The index of refraction in this region is increased and forms a multimode optical waveguide, the size of which varies directly with the applied voltage.

Figure 49 illustrates a modification of this concept which may prove superior in performance.(28) In this device light propagating in the waveguide formed by electrode strip $A$ can be coupled to the waveguide formed under strip $B$ by making the two induced waveguides overlap. The output from both channels can be fed to detectors for comparative processing. The voltages required are rather large, but an improvement in the voltage-coupling characteristic should be possible by using closer electrode spacing. Increasing the interactive length should also reduce the voltage required to achieve a given level of coupling.

Further improvement of the modulator-switch may be possible by forming permanent waveguides under the electrodes. One fabrication technique that appears particularly attractive is lowenergy electron bombardment of the waveguide region. Such an approach has been used successfully by Houghton and Townsend ${ }^{(29)}$ who investigated this technique as a possible extension of waveguide formation by ion bombardment. Their waveguides were formed in fused silica using electron energies between 11 and $16 \mathrm{keV}$. These waveguides began annealing at $400^{\circ} \mathrm{C}$, and it is possible that this temperature can be raised by proper selection of material and dose levels.

Several electro-optic materials are available for use, but lithium niobate appears to be the best yet reported. It has a relatively large electro-optic coefficient and dielectric anisotropy (different refractive indexes for different polarizations). The critical temperature of $\mathrm{LiNbO}_{3}$ is $1470^{\circ} \mathrm{K}\left(1193^{\circ} \mathrm{C}\right)$ and the melting temperature is $1250^{\circ} \mathrm{C}$. In general, for all materials that exhibit a phase transition, the electrooptic coefficients increase approximately as (dielectric constant)-1, until the transition temperature is reached, so that elevated temperatures down-hole should enhance the modulator operation. Other possible materials are lithium tantalate and barium sodium niobate.

\section{Optical Power Transmission}

Supplying electrical power down-hole via an optical beam and without electrical cabling may be feasible. There are two major aspects of this concept to be considered: (1) optical power transmission and (2) conversion of optical to electrical power. Crow(30) reported pulsed transmission of power up to 44 $\mathrm{MW} / \mathrm{cm}^{2}$ from a Nd glass laser over a $2 \mathrm{~km}$ fiber with measured linear (low power) losses of $5 \mathrm{~dB} / \mathrm{km}$. The high power loss was also $5 \mathrm{~dB} / \mathrm{km}$ indicating that no nonlinear losses were present. The transmitted peak power density of $44 \mathrm{MW} / \mathrm{cm}^{2}$ represents a total peak power in excess of $2100 \mathrm{~W}$ for the $78 \mu \mathrm{m}$ core diameter fiber which was used. The limiting condition of the experiment was not the power handling capability of the fiber, but the maximum power output of the laser. The maximum $C W$ power available for this experiment was $6 \mathrm{~W}$ which is far short of the $2100 \mathrm{~W}$ peak power. Thus, the $\mathrm{CW}$ experiment represents no limit at all since the limiting $C W$ power for low-loss fibers should approach the peak power limit. Crow also states that a broad line (i.e., the laser emission is spread out over as broad a spectrum as possible) source increases the overall power handling capacity of the fiber.

This ability of low-loss optical fibers to transmit high power optical beams opens the possibility of providing moderate amounts of electrical power through optical detection by semiconductor diodes. In particular, it has been demonstrated that efficiencies exceeding $18 \%$ for silicon solar cells and $22 \%$ for gallium arsenide solar cells can be achieved. For a CW source power of $100 \mathrm{~W}$, a $5-\mathrm{km}$ fiber with $10 \mathrm{~dB} / \mathrm{km}$ losses, and a detector with a modest $10 \%$ efficiency, the available down-hole power would be $0.1 \mathrm{~mW}$ at one $\mathrm{km}$, sufficient to power an optical modulator, since low data rates can be used. With a $1000 \mathrm{~W}$ laser 


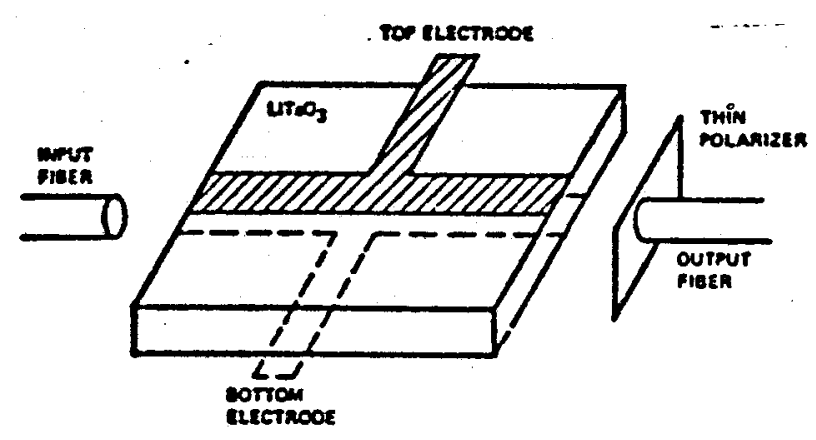

(a)

$\stackrel{\infty}{n}$

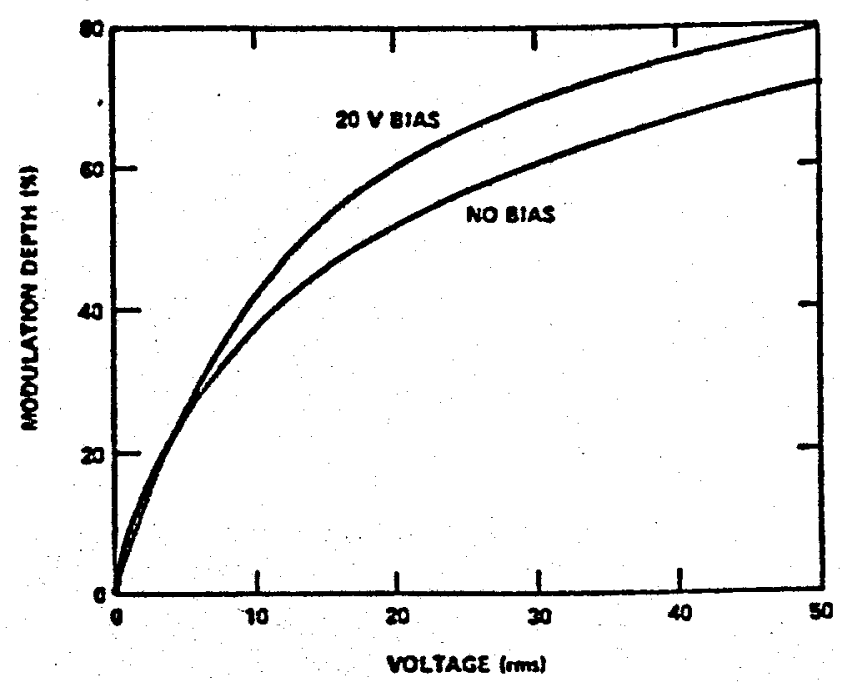

(b)

FIGURE 49. ELECTRO-OPTIC WAVEGUIDE MODULATOR, USING LiTaO 3 SLAB. a) Schematic of Modulator, b) Typical Modulation Versus Applied Voltage Response. 
under more favorable conditions, i.e., $5 \mathrm{~dB} / \mathrm{km}$ fibers (a reasonable goal) and $20 \%$ conversion efficiency, over $60 \mathrm{~mW}$ would be available to power either a source or a modulator.

\section{Pressure Sensor Investigation}

The literature review conducted during the program included consideration of techniques applicable to down-hole pressure measurement by optical means. Several modulation concepts described in Section $\mathrm{C}$ were found to be useful. One of the most promising of these is the frustrated internal reflection modulator.

This concept is illustrated in Figure 50, in which a pressure window is constructed in the wall of a pressure-tight logging housing, covered with a deflecting diaphragm (the deflection dimensions are extremely small). The diaphragm acts, under pressure, on one of several elements in which total internal reflection may occur. In the figure, a combination of prisms and three optic fibers is shown. The path of a single ray is shown as it enters the sensor from the central fiber. The solid line represents the situation when the diaphragm is not depressed, and therefore, there is a "large" space (a few wavelengths) between the corner reflector and a frustrating prism. The ray is totally internally reflected within the corner reflector and re-enters the input fiber. The dashed line illustrates the ray entering the prism as the diaphragm is deflected and the frustrating prism moves very close to the back surface of the corner reflector. Very little of the ray is then internally reflected, (dashed line) and most of it passes on into the frustrating prism where it is reflected by the silvered surfaces into one of two other fibers. The light carried by these additional collecting fibers can be compared with that returned via the first fiber to give a more accurate pressure indication and to compensate for variables in the system.

The dynamic range of this device can be controlled by several parameters. In particular, the stiffness of the diaphragm controls the overall sensitivity, a stiffer material providing a greater dynamic range. A less stiff material would provide greater resolution over a limited range. For a given material, and particularly for the more flexible ones, the limits of the available dynamic range can be adjusted by varying the at rest ( 0 psig) position or by pressurizing the device. The latter may also affect the dynamic range itself, but may be desirable to provide approximately equal internal and external pressures in the operating environment.

Another sensor concept makes use of the coupling, or crosstalk, between two "parallel" fibers via their evanescent fields. If two unclad sections of fiber are placed in close proximity as shown in Figure 51 a where " $d$ " is the separation and " $D$ " the length of the parallel sections, the intensity profile of the light entering fiber " $A$ " will be as shown in Figure 51b. As the light travels through fiber " $A$ " some of its is transferred to fiber " $B$ " because the electromagnetic field of the light wave extends beyond the physical limit of the fiber and, in fact, overlaps fiber B. The result is shown in Figure $51 \mathrm{c}$ where both fibers are carrying light. The amount of light coupled into fiber " $B$ " depends upon both the coupling length, " $D$ ", and the separation, " $\mathrm{d}$ ". For the applications we are considering, the desired "modulation" may be achieved by using a fixed coupling length and varying the spacing.

Such a sensor was devised by $\mathrm{Kane}^{(31)}$ as early as 1966 , but was not suitable for very remote measurements because suitable low-loss optical fibers were not available to carry the light over extended distances. Kane took advantage of beat modes that are created by the interaction of the paired modes, which are always present with coupled waveguides. He showed that through careful analysis of changes in these beat modes caused by changes in the distance $d$, it is theoretically possible to detect displacements as small as $0.0003 \mathrm{~nm}$, if one ignores thermal and quantum mechanical constraints. Although such constraints will undoubtedly lead to poorer sensitivity even under ideal conditions, Kane's prediction suggests that the idea is worthy of further consideration. 


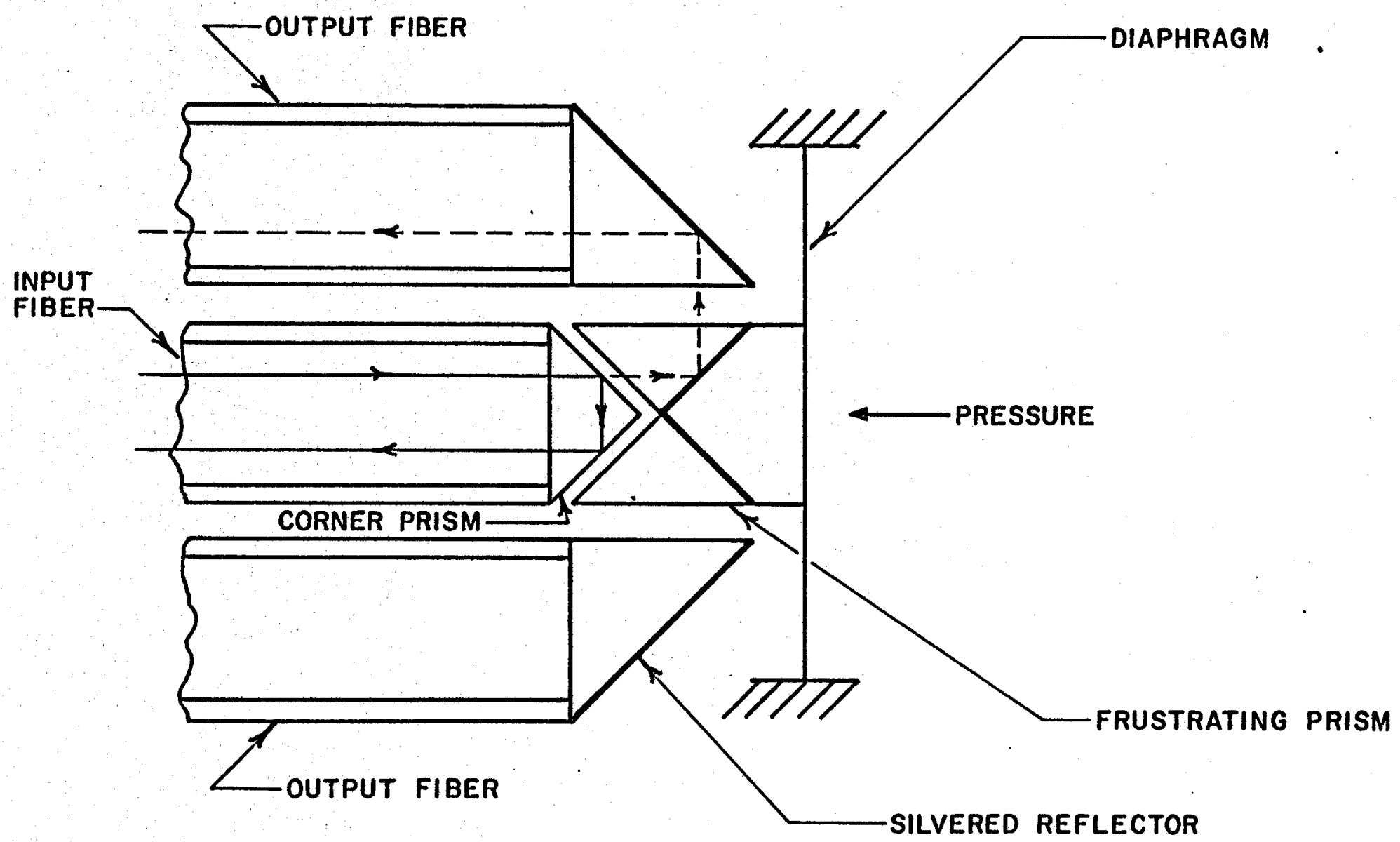

FIGURE 50. PRESSURE TRANSDUCER EMPLOYING FRUSTRATED TOTAL INTERNAL REFLECTION 


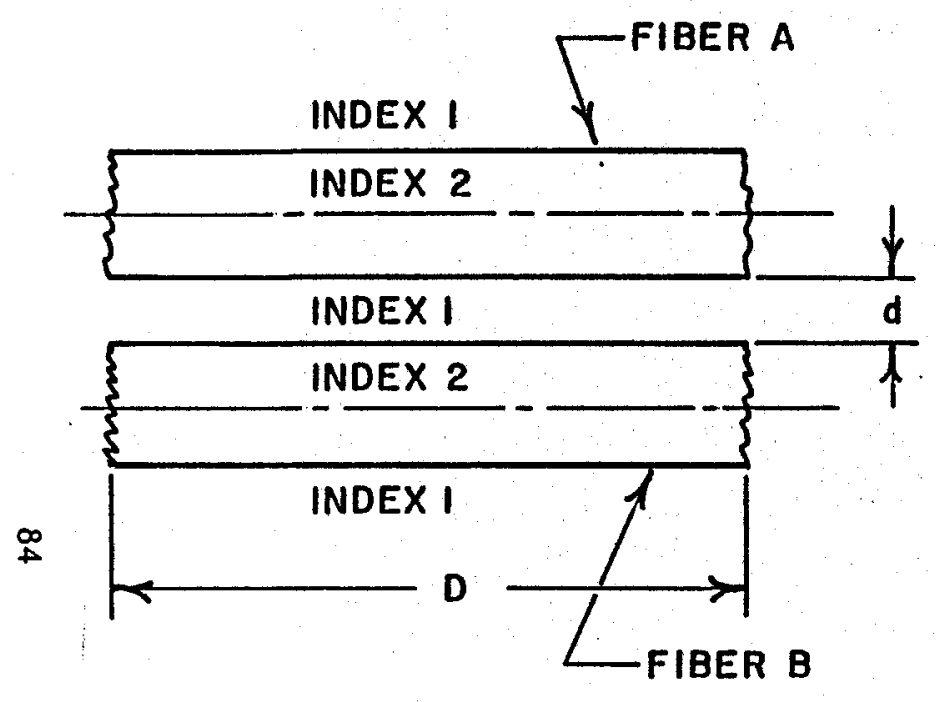

a. Fiber configuration

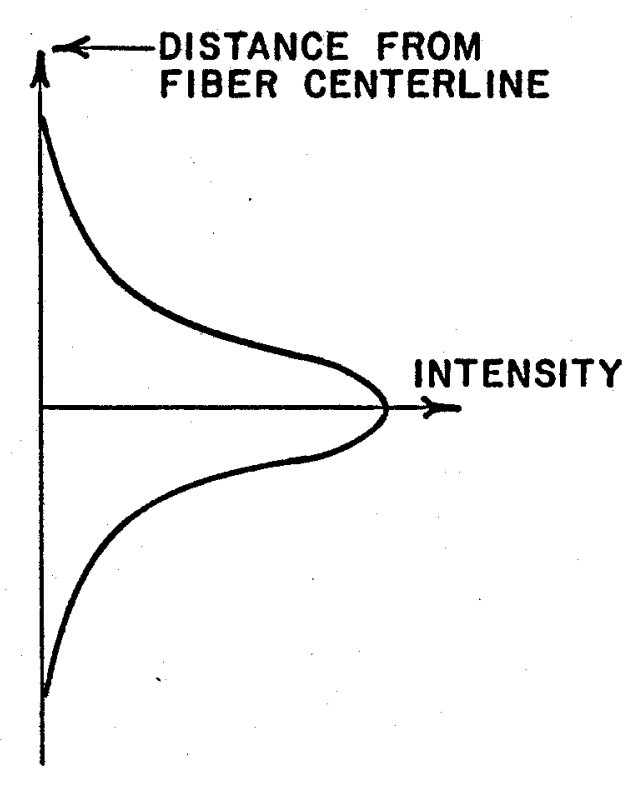

b. Intensity field of Fiber A

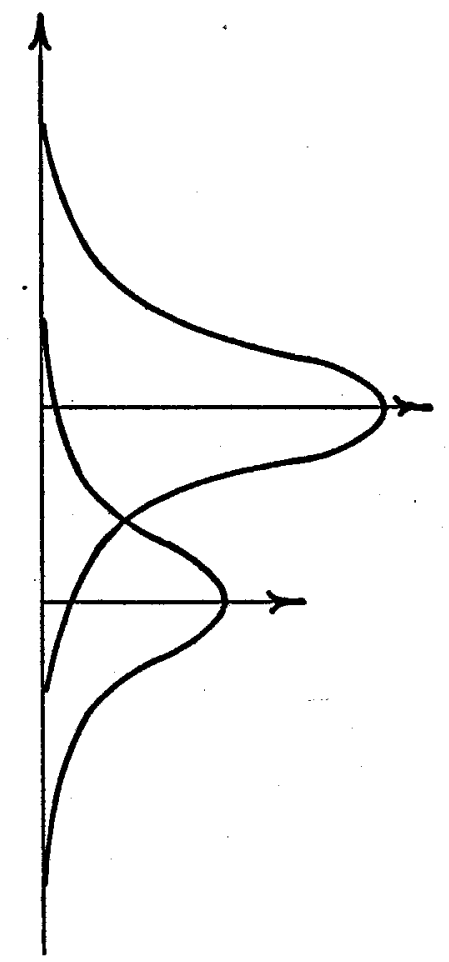

c. Intensity fields of Fibers $A$ and $B$ showing cross coupling

FIGURE 51. TWO-FIBER DISPLACEMENT SENSOR EMPLOYING EVANESCENT COUPLING 


\section{CONCLUSIONS AND RECOMMENDATIONS}

\section{A. Summary of Results}

The basic components of an optical well-logging system have been investigated, and major problems associated with the implementation of such a system have been defined.

\section{Optical Cable}

The design of an armored well-logging cable was accomplished, and construction efforts made. Difficulties associated with the application of high-temperature buffer material of sufficient thickness on quartz fibers were found to be very severe. The conventional cabling of such fibers for a high pressure environment makes use of the imbedment of fibers in soft, resilient plastic and plastic foam. No satisfactory substitute for this treatment suitable for high temperatures was found. A reasonably successful approach involved the isolation of the fibers by a thin layer of nitrile rubber under a heavy buffer layer of PFA. This technique, however, falls short of the temperature goal of $275^{\circ} \mathrm{C}$. Major problems in cable development remain.

\section{Associated Optical Components}

The investigation of optical waveguides, sources, couplers, modulators, pressure connectors, and principal associated system hardware items disclosed no serious limitations or constraints. Although representing considerable developmental effort, implementation of a successful optical temperature logging system should be straightforward assuming success in the construction of a satisfactory optical cable.

\section{Optical Sensors}

Optical sensor concepts related to temperature, neutron, and pressure logging operations were investigated, and the temperature and neutron sensors evaluated experimentally.

a. Temperature Sensor. A temperature logging sensor, based on the fundamental optical absorption edge in semiconductors, was constructed and tested. Two approaches to surface demodulation of the optical signal were explored, and a method based on photomultiplier threshold detection of the transmission spectrum implemented in the laboratory.

b. Neutron Logging Sensor. A thermal neutron detector based on optical pumping of a YAC crystal by an alpha-sensitive $\mathrm{ZnS}$ phosphor was experimentally evaluated. Alpha particles, produced by the $B^{10}(n, q)$ reaction, produce a broad spectrum of radiant energy in $\mathrm{ZnS}$. This energy is largely within the absorption bands of Nd:YAG, which strongly reemits in a narrow band of wavelengths near $1.06 \mu \mathrm{m}$, suitable for direct transmission over quartz fibers. Investigation of an alpha detector based on this principal was conducted in the laboratory.

\section{B. Conclusions}

The following conclusions can reasonably be drawn:

- Development of a high-temperature optical cable presents somewhat similar materials problems to those of high-temperature electrical cable. However, the elimination of electrical insulation requirements may present opportunities for other types of optical waveguide protection. The subject of high-temperature cable construction is far more complex than originally envisioned, and still represents a major developmental effort in an optical well-logging 
system. The promise of non-electronic, passive down-hole systems, however, appears to make the development well worthwhile. Well-logging requirements at temperatures of $400^{\circ}-500^{\circ} \mathrm{C}$ may offer no reasonable alternative approach.

- Passive optical temperature sensors and neutron logging sensors appear entirely feasible, with ultimate temperature capabilities above $500^{\circ} \mathrm{C}$. Limitations are confined to mechanical constructional details such as adhesives, optical coupling, and pressure seals.

- Extension of optical measurement techniques to pressure, acoustic (seismic) and other non-electric parameters appears to be a reasonable research expectation.

\section{Recommendations}

The following recommendations follow the results of this study:

- Major effort should be applied to the construction of an optical cable. The problems have been defined, and research should be devoted to these problem areas, with confidence and full expectation of eventual success.

- Implementation of an optical temperature logging system should be initiated, with the development of a computer simulation program for the complete system for use as a tool in the study of component requirements. This program should accept, as input, numerical values for the power density entering the fiber, cable and coupler losses, crystal absorption coefficient data, crystal thickness, and each of the other parameters involved. Output should include the transmitted power density and photomultiplier response as a function of wavelength, and the value of the threshold wavelength. In short, the program should provide the capability to predict system performance for any combination of values of component data. As a check on the reliability of such predictions, laboratory experiments, much like those recently completed with GaAs, should be performed for comparison with the results of computations.

- Continued research in the optical sensor area should be pursued, with demonstration of the complete optical neutron logging sensor, development of optical pressure sensor concepts, and continued evaluation of new applications. 


\section{- BIBLIOGRAPHY}

1. Kato, D., "Light Coupling from a Stripe-Geometry GaAs Diode Laser into an Optical Fiber with Spherical End," J. Appl. Phys., Vol. 44, pp. 2756-2758, June 1973.

2. Miller, Steward E., Marcatili, E.A.J., Tingye Li, "Research Toward Optical-Fiber Transmission Systems," Proc. IEEE, Vol. 61, pp. 1703-1751, Dec. 1973.

3. Koiziemi, K, et al., "New Light-Focusing Fibers Made by a Continuing Process," Appl. Opt., Vol. 13, pp.255-260, Feb. 1974.

4. Tsuchiya, H. et al., "Double Eccentric Connectors for Optical Fibers," Appl. Opt., Vol. 16, pp. 1323-1331, May 1977.

5. Baker, L.E., et al., (eds.), "Report of the Ceophysical Measurements in Geothermal Wells Workshop," Sandia Labs, SAND 75-0608, Dec. 1975.

6. Compton, W.D. and Rabin, H., "F-Aggregate Centers in Alkali Halide Crystals," Solid State Phys. 16, 121. (1964).

7. Tomiki, T., "Photo-and Thermochemical Reactions in Potassium Chloride Crystals-A Quantitative Study of the M Centre," J. Phys. Soc. Japan, 15, 488 (1960).

8. Jain, S.C. and Jain, V.K., Phys. Rev. 181, 1312 (1969).

9. Seitz, F., Rev. Mod. Phys. 26, 7 (1954).

10. Pankove, J.I., "Optical Processes in Semiconductors," Dover Publications, Inc., New York (1975).

11. Calleja, J.M. and Agullo-Lopez, F., Phys. Stat. Sol. (a), 25, 473 (1974).

12. Moss, T.S., “Optical Properties of Semiconductors," Butterworths, London (1961).

13. Bilenko, D.I. and Tsiporukha, V.D., Sov. Phys. Semi-cond. 8, 804 (1974).

14. Gershenzon, M., in "Semiconductors and Semimetals Vol. 2," R.K. Willardson and A.C. Beer, eds., Academic Press, New York (1966).

15. Johnson, E.J. in "Semiconductors and Semimetals Vol. 3," R.K. Willardson and A.C. Beer, eds., Academic Press, New York (1967).

16. Redfield, D. and Afromowitz, M.A., Appl. Phys. Letters 11, 138 (1967).

17. Jain, S.C. and Jain, V.K., Phys. Rev. 181, 1312 (1969).

18. Gershenzon, M., in "Semiconductors and Semimetals Vol. 2," R.K. Willardson and A.C. Beer, eds., Academic Press, New York (1966).

19. Sun, K.H., Malmberg, P.R., and Pecjak, F.A., Nucleonics 14, 46 (1956).

20. Curie, D., "Luminescence in Crystals," John Wiley \& Sons, Inc., New York (1963). 
21. Kroger, F.A., "Some Aspects of the Luminescence of Solids," Elsevier, Amsterdam (1948).

22. Gross, G.E., Phys. Rev. 116, 1478 (1959).

23. Geusie, J.E., et al., "Laser Oscillations in Nd-Doped Yttrium Aluminum Garnets," App. Phys. Letters, Vol. 4, No. 10, pp. 182-184.

24. Koningstein, J.A.. and Geusie, J.E., "Energy Levels and Crystal Field Calculations in Nd ${ }^{3+}$ In YAG," Phys. Rev., Vol. 136, pp. A711-716 (1964).

25. Price, W.J., "Nuclear Radiation Detection," McCraw Hill, New York (1958).

26. Burch, C.R., "Reflecting Microscopes," Proc. Phys. Soc., Vol. 49, pp. 41-46, (1947).

27. Burch, C.R., "Semi-Aplanat Reflecting Microscopes," Proc. Phys. Soc., Vol. 49, pp. 47-49, (1947).

28. Soref, R.A., McMahon, D.H., and Nelson, A.R., Appl. Phys. Letters 28, 716 (1976).

29. Houghton, A.J. and Townsend, P.D., Appl. Phys. Letters 29, 565 (1976).

30. Crow, J.D., "Power Handling Capability of Glass Fiber Lightguides," Appl. Opt., Vol. 13, p. 467, Mar. 1974.

31. Kane, J., IEEE Trans. Geoscience Elect. GE-4, 1 (1966). 


\section{APPENDIX A}

\section{ANALYSIS OF TEMPERATURE SENSOR EXPERIMENTS - MAXIMUM SLOPE METHOD}

\section{A. Data Acquisition}

Figure $A 1$ is a block diagram of the data acquisition system, in which the optical equipment was linked to a Hewlett Packard HP2100 computer system. An analog voltage proportional to the wavelength was produced by attaching a $1 \%$ resistance voltage divider to the monochromator. The analog voltages representing the wavelength and transmitted intensity were interfaced to the HP2100 via a 10-bit analogto-digital converter. A flow chart of the program is shown in Figure A2. When data transfer is complete, data are stored on a magnetic tape cartridge in a Tektronix 4051.graphics terminal

The data reduction is performed on the Tektronix 4051 via a multiplot program. This program is a modification of the $x-y$ plot program supplied with the system. A flow chart of this program is given in Figure A3. The operation centers around a listing of options called the "menu". These options are:
1. Enter Data
9. Set $y$ data range
2. Display Data
10. Select plot mode
3. List Data
11. Select symbol
4. List Parameters
12. Insert data
5. Set autoscale
13. Delete data
6. Set $x$ screen position
14. Change data
7. Set y screen position
15. Store data
8. Set $x$ data range
16. Stop

Selection of Option 1 allows one to enter data from the keyboard or magnetic tape. Option 3 lists the data in a table, etc. Selection of Option 2 leads to the area of main modification. This was the addition of optional methods of displaying the data. Selection of Option 1 results in a plot of raw data as in Figure A4. Selection of Option 2 results in Figure $A 5$ in which the calibration response shown in Figure $A 6$ has been divided out. Option 3 uses Equations (A3) and (A5) to plot the absorption coefficient as in Figure A7. Finally, Option 4 uses Equation (A6) to plot the slopes as in Figure A8. Other slight modifications were also made to the original program, e.g. labeling of the plot axes. However, the graphic part of the program is essentially as supplied by Tektronix.

\section{B. Data Reduction}

The actual voltage output from the experimental set up includes not only the effect of the GaAs, but the effect of the source and all other optical components as well. Therefore, a calibration run was made without the GaAs in place, and the effect of the CaAs could be separated by dividing by this calibration. Thus the transmission coefficient is

$$
T=\frac{I}{I_{0}}=\frac{V \text { actual }}{V \text { calibration }}
$$




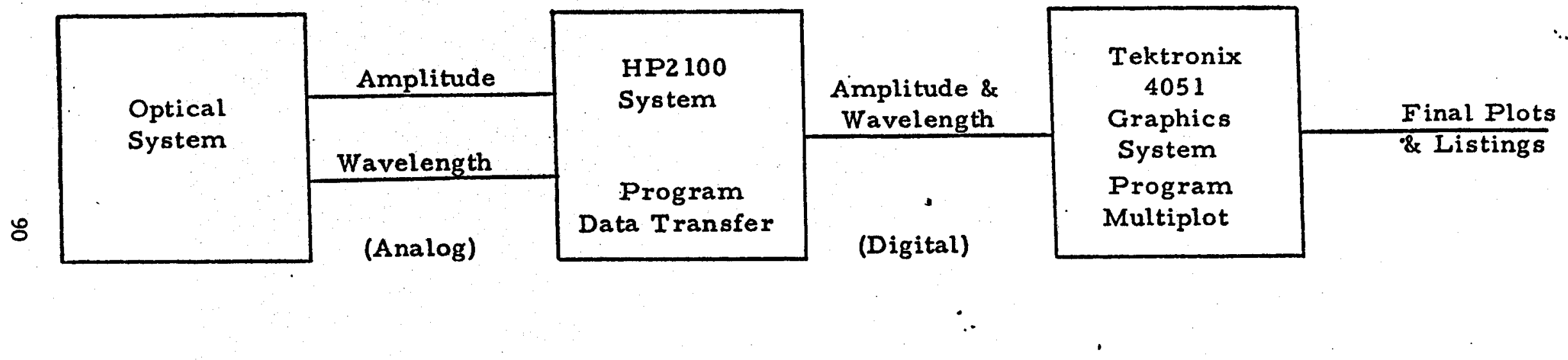

FIGURE A1. BLOCK DIAGRAM OF EXPERIMENTAL DATA ACQUISITION SYSTEM 



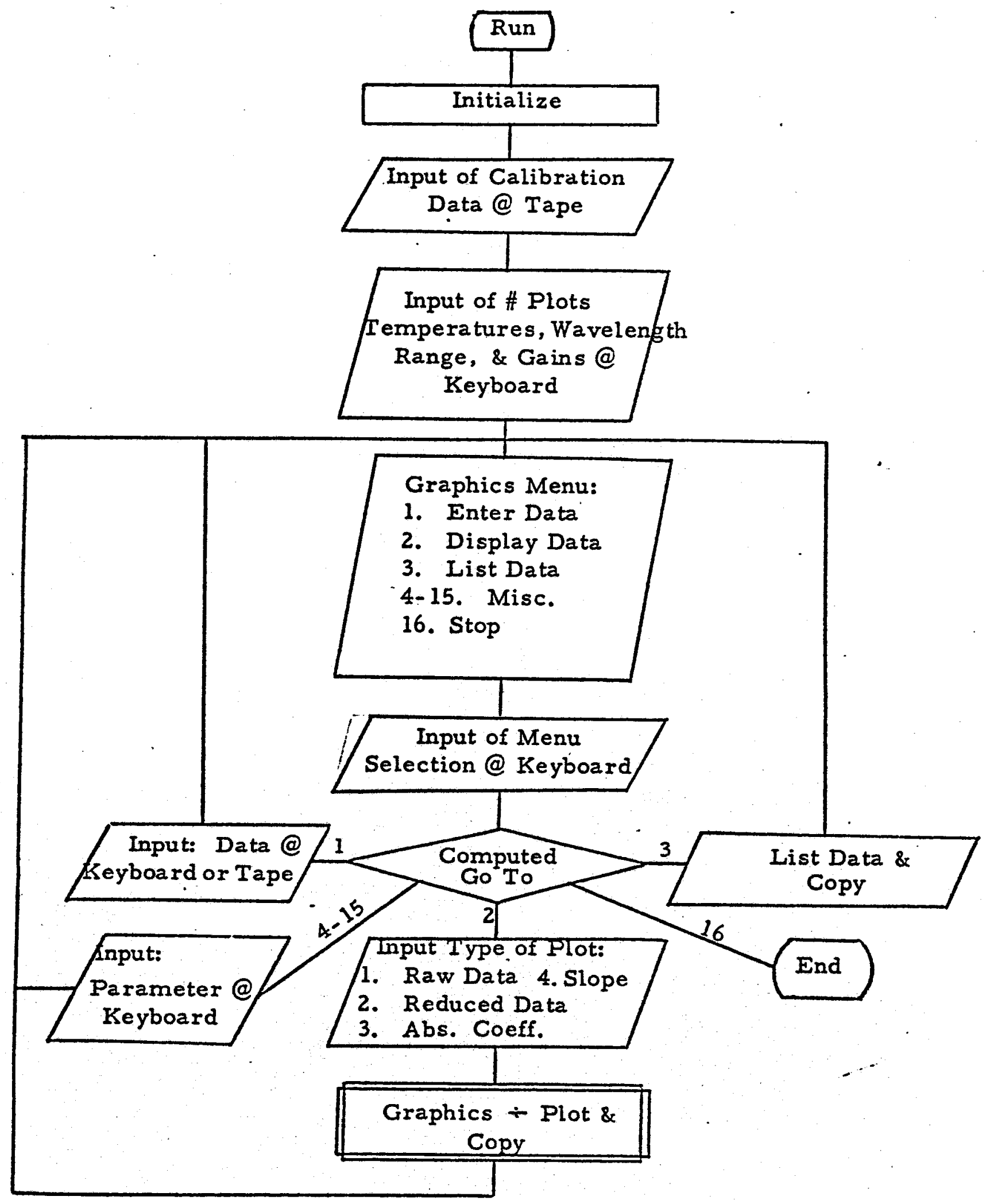

FIGURE A3. FLOW CHART FOR MULTIPLOT PROGRAM 


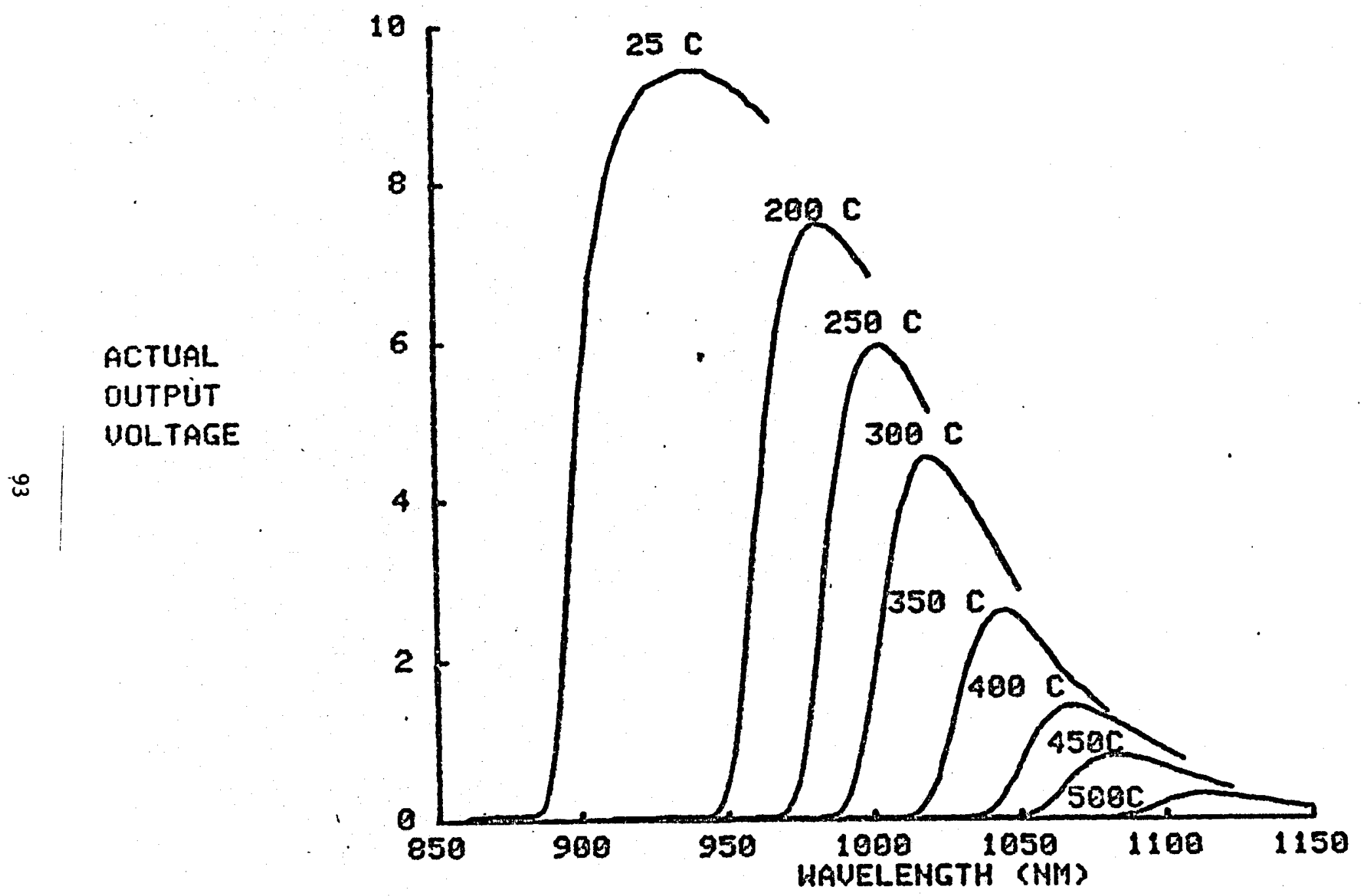

FIGURE A4. RAW DATA FOR TEMPERATURE SENSOR EXPERIMENTS USING THE ORIGINAL GALLIUM ARSENIDE CRYSTAL 


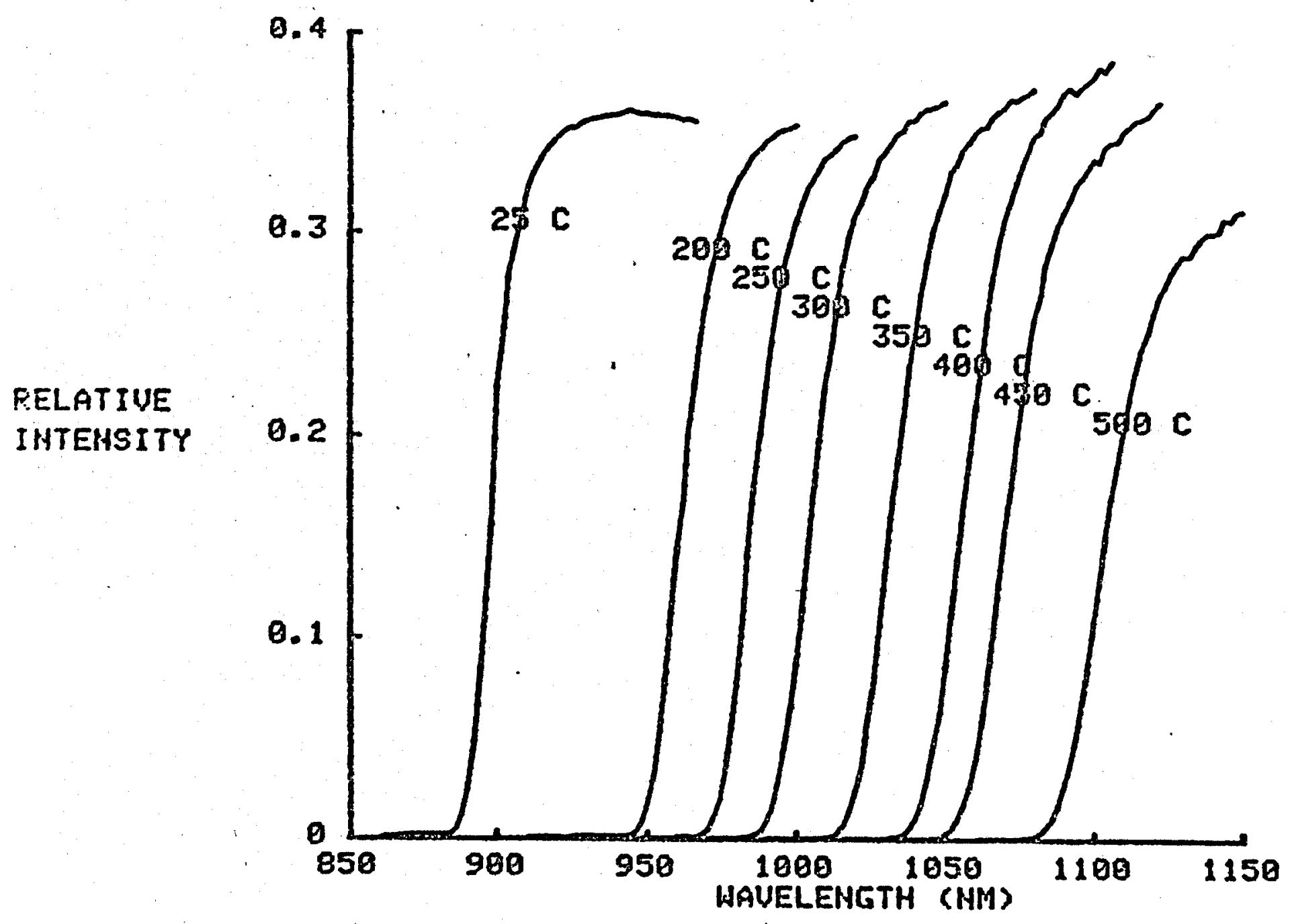

FIGURE A5. REDUCED DATA FROM TEMPERATURE SENSOR EXPERIMENTS USING THE ORIGINAL GALLIUM ARESENIDE CRYSTAL 


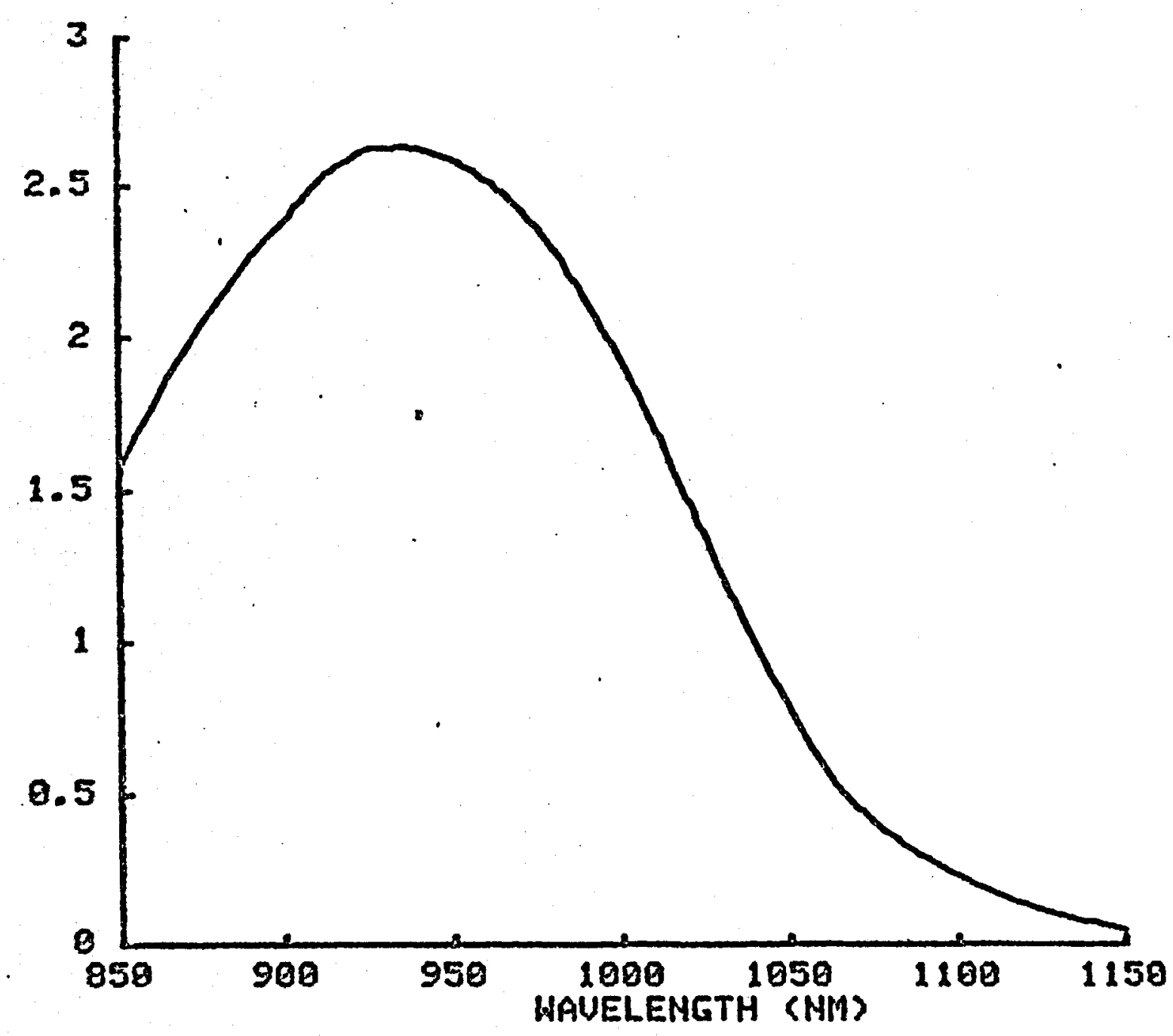

FIGURE A6. CALIBRATION DATA FOR TEMPERATURE SENSOR EXPERIMENTS 


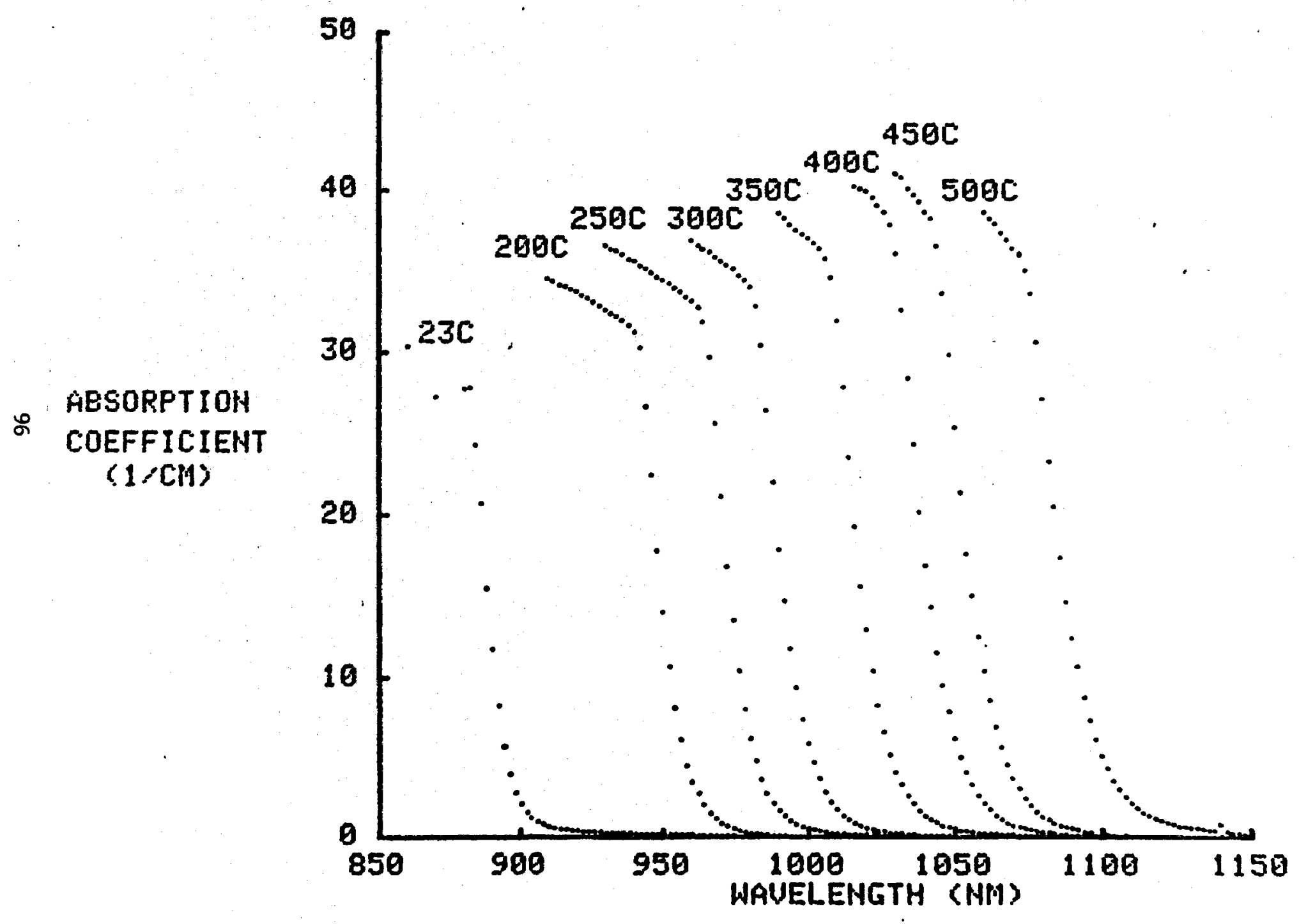

FIGURE A7. MEASURED ABSORPTION EDGE FOR GaAs AS A FUNCTION OF TEMPERATURE, CORRECTED FOR REFLECTION. 


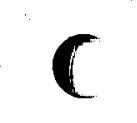


To compare and understand the results of the experiments in the context of information in the literatue, various optical constants of GaAs were calculated. Assuming a parallel optical interface, the following equation for transmission of light ${ }^{(1)}$ applies

$$
T=\frac{I}{I_{0}}=\frac{(1-R)^{2}}{e^{\alpha D}-R^{2} e^{-\alpha D}}
$$

where $R$ is the reflectivity, $D$ is the thickness, and $\alpha$ is the absorption coefficient. Solving for the absorption coefficient one obtains:

$$
\alpha=\frac{1}{D} L N\left[\frac{(1-R)^{2}}{2 T}\left(1 \pm \sqrt{1-\frac{4 R^{2} T^{2}}{(1-R)^{4}}}\right)\right]
$$

The Tektronix 4051 was programmed to evaluate this expression and display an experimental absorption coefficient. First however, a value for the reflectivity must be determined.

The reflectivity was determined by analyzing the transmission data in the region where there was no absorption, thus

$$
T=\frac{I}{I_{0}}=\frac{(1-R)}{1+R}
$$

Figure A9 shows the result of this analysis for temperatures of $23^{\circ} \mathrm{C}$ to $500^{\circ} \mathrm{C}$. Note that the inadvertent deposition of material (tin) on the surface of the sample during the experiment at high temperature is very evident in this plot. A fit to the data for temperatures in the $200-400^{\circ} \mathrm{C}$ range was made and is also plotted in Figure A9. This equation is

$$
R=0.777-0.000297 \lambda
$$

where $\lambda$ is wavelength in nanometers. The curve fit compares favorably with reported values of about 0.3 for electropolished crystals.

Equations (A3) and (A5) were used to plot the experimental data as an experimental absorption coefficient (the data plotted in this format was shown in Figure A7). The slope of this experimental absorption coefficient can be calculated by using the linear approximation from point to point, i.e.

$$
\text { Slope }=\frac{\alpha(n+1)-\alpha(n)}{\lambda(n+1)-\lambda(n)}
$$

where $\mathrm{n}$ is arbitrary point number. This slope is assumed to be the slope at the midpoint, i.e., at

$$
\lambda=[\lambda(n+1)+\lambda(n)] / 2
$$

The plot of the experimental slopes of the absorption edges was shown in Figure AB.

\section{Data Analysis}

In a wide band gap semiconductor such as gallium arsenide, the absorption is cut off above some definite wavelength since photons of higher wavelength do not have enough energy to excite the carriers from the valence to the conduction band. The equation for the absorption coefficient around this absorption edge, known as Urbach's rule, is

$$
\alpha=\frac{\alpha_{0}}{1+\exp [\gamma(1 / \lambda g-1 / \lambda)]}
$$




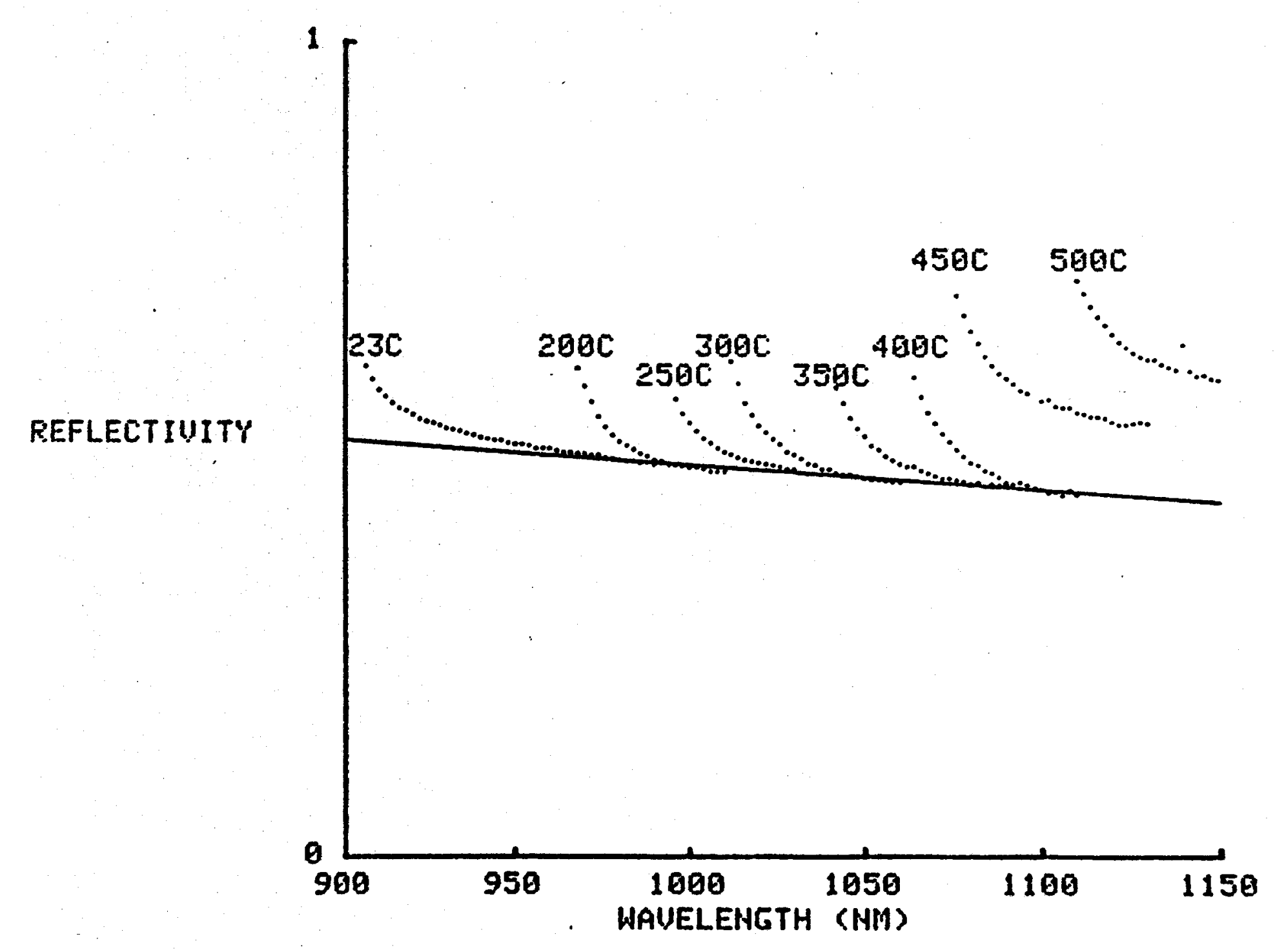

FIGURE A9. EXPERIMENTAL DET ERMINATION OF REFLECTIVITY AS A FUNCTION OF WAVELENGTH FOR VARIOUS VALUES OF TEMPERATURE, GaAs 
where $\alpha_{0}$ is the low wavelength limit, $\lambda_{g}$ is the cutoff wavelength, and $\gamma$ is the "steepness" of the cutoff. Thus, the problem was to analyze the experimental data to determine the three unknowns: $\lambda g$. , and 0 . (The reflectivity was previously specified in Equation A5.)

The cutoff wavelength was found by analyzing the slope of the absorption coefficient. Differentiating Equation $\mathbf{A} 8$ gives

$$
\frac{d \alpha}{d \lambda}=\frac{-\alpha_{0} \gamma \exp [\gamma(1 / \lambda g-1 / \lambda)]}{\lambda^{2}\{1+\exp [\gamma(1 / \lambda g-1 / \lambda)]\}^{2}}
$$

This slope is a maximum at the cutoff wavelength. The values of the cutoff wavelengths for the various temperatures are given in Table A1. A fit was made to this data using the band gap as an adjustable parameter.

The low wavelength limit was calculated using the fact that at the cutoff wavelength the absorption coefficient is one-half of the low wavelength limit. The results of this calculation are given in Table A1. A discrepancy with reported values is seen here. The reported values of the low wavelength absorption coefficient are of the order of $10^{4} \mathrm{~cm}^{-1}$, a factor of 200 higher than our measured values. This discrepancy is one that requires resolution and effort has been directed at that question as discussed in the text.

\section{TABLE A1}

$\begin{array}{cccc}\text { Temperature } & \frac{\lambda_{g}(\mathrm{~nm})}{23^{\circ} \mathrm{C}} & \frac{\alpha_{0}\left(\mathrm{~cm}^{-1}\right)}{31} & \frac{\gamma(\mathrm{nm})}{265000} \\ 200^{\circ} \mathrm{C} & 946 & 44 & 190000 \\ 250^{\circ} \mathrm{C} & 968 & 47 & 181000 \\ 300^{\circ} \mathrm{C} & 986 & 48 & 179000 \\ 350^{\circ} \mathrm{C} & 1012 & 50 & 179000 \\ 400^{\circ} \mathrm{C} & 1036 & 50 & 180000 \\ 450^{\circ} \mathrm{C} & 1049 & 55 & 179000 \\ 500^{\circ} \mathrm{C} & 1081 & 51 & 177000\end{array}$

The steepness of the cutoff was also found by analyzing the slope of the absorption: coefficient. Evaluating Equation (A5) at the cutoff wavelength and solving for the steepness,

$$
\gamma=\left.\frac{4 \lambda \mathrm{g}}{\alpha_{0}} \frac{\mathrm{d} \alpha}{\mathrm{d} \lambda}\right|_{\lambda g}
$$


Thus, one has to use the low wavelength limit of the absorption coefficient from (A10)and cutoff wavelength from (A8) in order to calculate the steepness. The results of this calculation are given in Table A1 and plotted in Figure A. These values are of the same order of magnitude as reported values, but do not exhibit the usual dependence on temperature. The following equation is the normal means of explaining the exponential tail steepness,

$$
\gamma=\frac{2 \sigma}{\hbar \Omega 0} \tanh \left(\frac{\hbar \Omega 0}{2 K_{B} T}\right)
$$

where $\tau$ is a constant, $\approx$ is the optical phonon frequency and $T$ is the temperature. The curve in Figure A10 is a plot of Equation A11, showing that at room temperature the steepness may follow the theoretical expression, apparently a constant at higher temperatures. 


\section{FIGURE AI0 STEEPNESS}

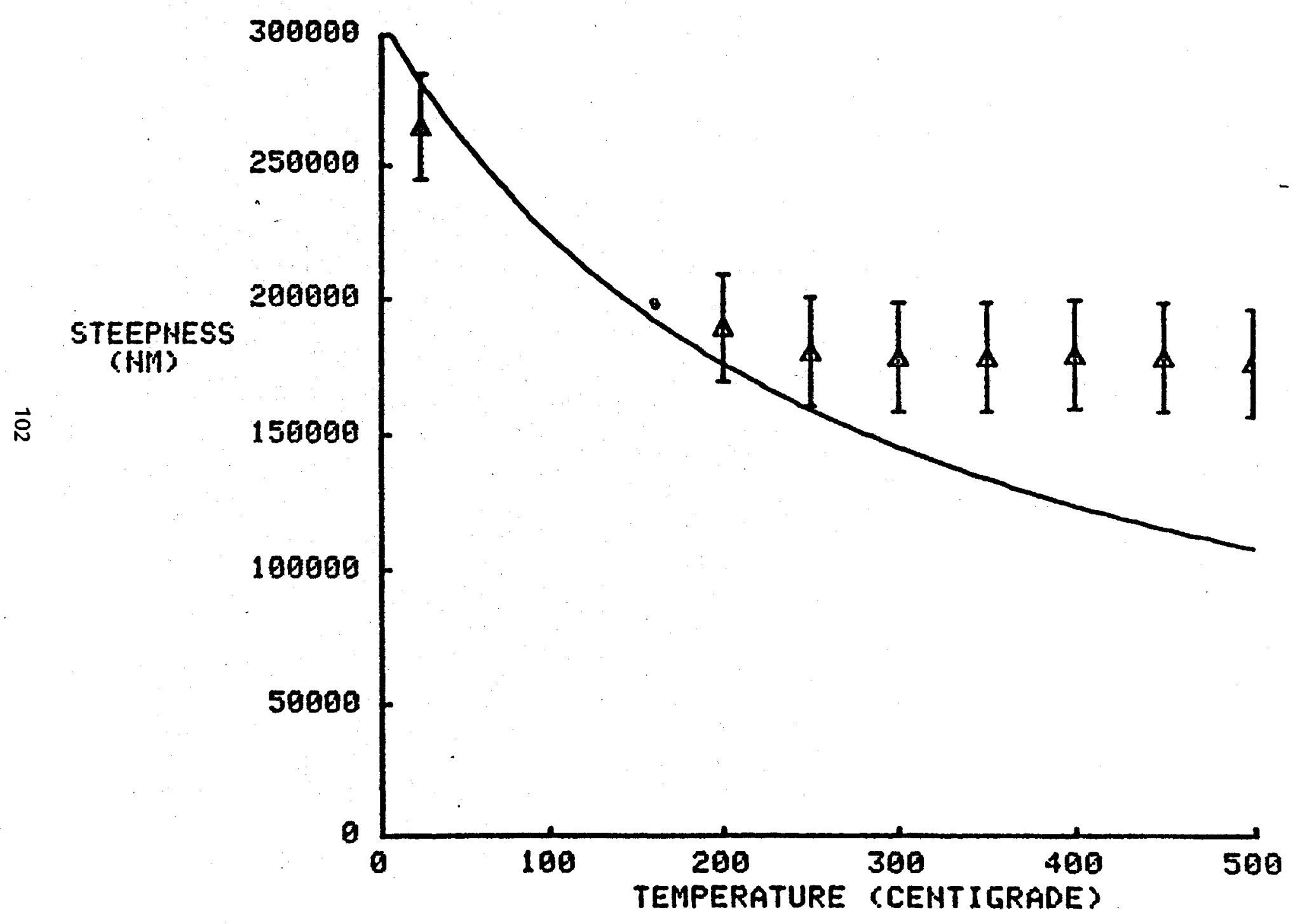




\section{APPENDIX B}

\section{OPDEAN, A RAY TRACE COMPUTER PROGRAM}

In the analysis of fiber-to-fiber connectors, and source-fiber or sensor-fiber couplers, it is useful to have available an algorithm for the prediction of coupling efficiencies of proposed designs. For this purpose we have obtained, from McDonnel Douglas Automation Center, the ray trace computer program OPDEAN. This program is written in FORTRAN IV for use on the CDC Series 6600 computers.

The program traces the trajectories of up to 800 rays from an object point through a specified system of lenses and mirrors to a set of image planes, the number and location of which are also specified by the user. A simple example is illustrated in Figure B1. The object point in this case is the point $\mathrm{O}$, the point of origin of each ray. Surfaces 1 and 2, which in this case are planes, define a region where the index of refraction differs from that of the surrounding medium. The planes labeled $I_{1}, I_{2}$, etc., are the image planes.

In general, surfaces that define regions where the index of refraction changes may be quardic or deformed quardic surfaces, and can be specified in coordinate systems that are arbitrarily tilted and/or decentered with respect to a fixed reference system. Input data include values of the parameters needed to specify the number and location of object points, the number and directions of rays emanating from each object point, the locations and shapes of all surfaces, and the number and locations of image planes. Output includes the coordinates of the point of intersection of each ray at each surface in the system, including all image planes, and the direction cosines or each ray at each surface.

To use the program for coupling efficiency calculations, we use an input option in which the program computer initial ray direction is such that each ray lies at the center of an angular sector of equal solid angle within a core of specified half angle. The fraction of the total number of rays that exit at positions and with directions appropriate to fiber optic propagation is then the efficiency for coupling to an optical fiber. The output data that determines whether a ray will couple to a fiber that terminates at an image plane are the off-axis distance of the ray in that plane and the direction cosine of the ray with respect to the axis of the fiber. If, for a particular ray, the off-axis distance is less than the fiber core radius and the direction cosine is greater than the minimum value for fiber propagation, then that ray will propagate. If one or both of these conditions are not met, then the ray will not propagate. Thus, with reference to Figure B1, rays 1 through 7 enter the fiber on the right while rays 8 through 11 do not.

Because several image planes can be specified in a single problem, one can use the output from one computer run to determine efficiencies as functions of the location of the output fiber. Thus, in the problem illustrated in Figure B1, data were generated for 7 image planes, corresponding to output fiber distances from 0 to $300 \mu \mathrm{m}$ from surface 2. The results are given in Table B1, along able for this simple configuration. As the data show, a 150 ray approximation to the coupling efficiency is adequate. Incidentally, the computer time required for this analysis was about 2 seconds on the CIBER 74 computer.

This initial test of OPDEAN, as well as other applications discussed elsewhere, shows that the program is capable of accurate and efficient computations of coupling efficiencies for systems involving point sources and quardic or deformed quardic surfaces. However, it is not well suited to the accurate - simulation of distributed sources, such as the light emanating from the end of a fiber. With the program in its present form this would require repeated application for a number of off-axis point sources, each of which represents the radiation from an element of area on the distributed source. Analysis discussed in a preceding section of the report showed that this process is extremely tedious and inefficient. It is therefore worthwhile to consider modifications of OPDEAN to handle those distributed source problems that occur most frequently in fiber coupling studies. 
4727

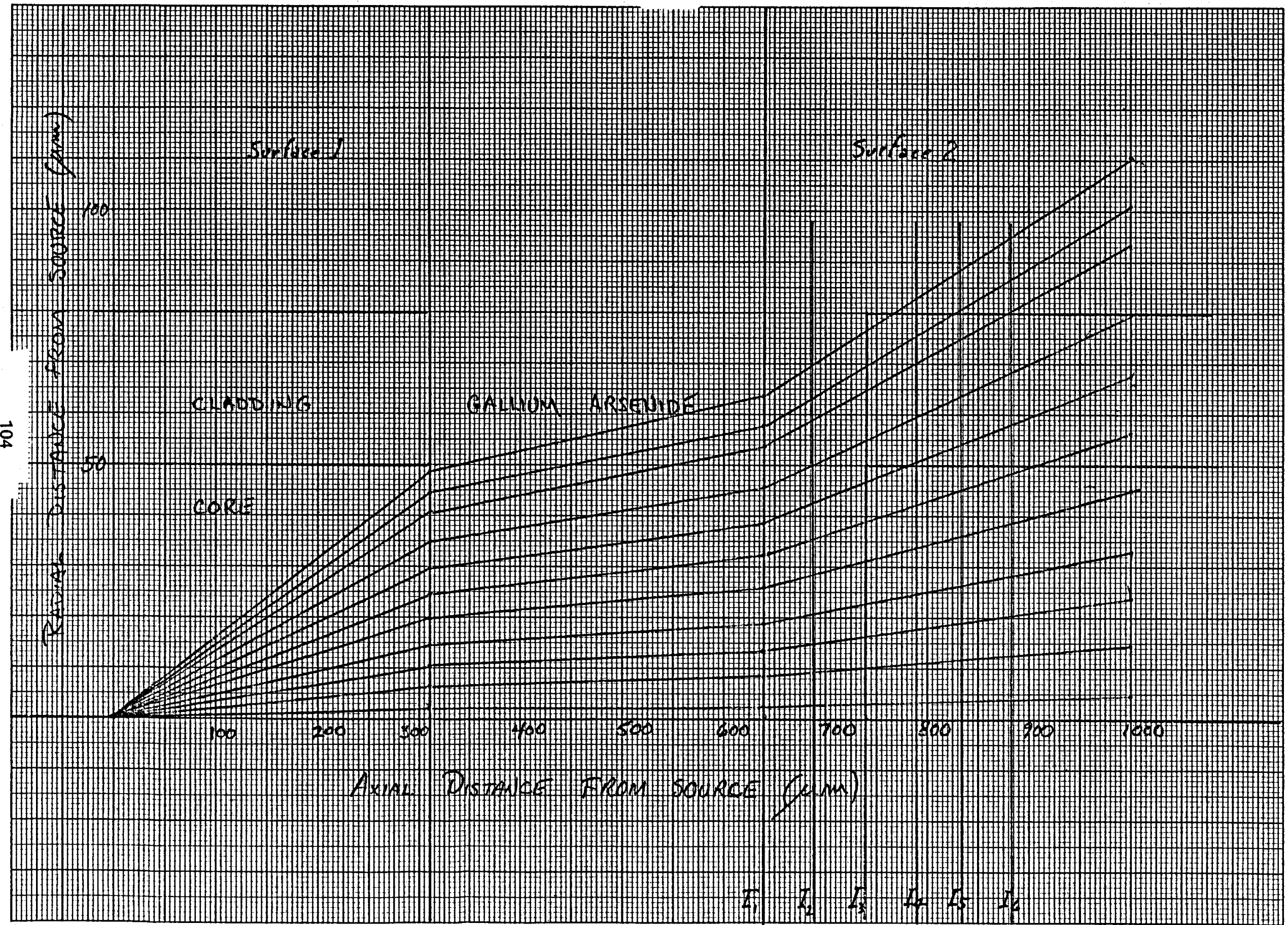


In the applications that we foresee, the principal distributed source problem will probably be that of specifying a representative distribution of ray positions and directions corresponding to radiation leaving the end of a fiber. One way that this can be accomplished is to add to OPDEAN a subroutine that uses, as input data, the position and direction of each ray from a point source, and returns, to the main program, the new position and direction of the ray after it has undergone multiple reflections in propagating down a long cylinder with reflecting walls. If this subroutine is inserted after OPDEAN computes the ray positions and directions in the first (entrance pupil) plane, but before the program enters the rest of the ray trace routine, then the subroutine will, in effect, replace the original point source ray distribution with the "scrambled" distribution caused by multiple reflections in an optical fiber.

In analyzing this problem of multiple reflection in a cylinder, we have developed an exact solution for the exit position and direction of a ray in terms of the length and diameter of the cylinder, and the position and direction cosines of the ray as it enters the fiber. Mathematical details, a listing of the subroutine, and the results of a few sample computations are given below. Addition of this subroutine to OPDEAN has not yet been attempted.

The problem of concern is illustrated in Figure B2. We are given the coordinates $x_{0}, Y_{0}$ and direction cosines $q_{x 0^{\prime}} q_{y o}, q_{z o}$ of a ray as it enters the end of a reflecting cylinder of length $l$ and radius $a$. We wish to calculate the coordinates and direction cosines of the ray as it leaves the cylinder in the plane $z 4^{1} \ell$.

From the equation of the line through the entrance point

$$
\frac{x-x_{0}}{q_{x 0}}=\frac{y-y_{0}}{q_{y_{0}}}=\frac{z}{q_{z 0}}
$$

and the equation of the cylinder

$$
x^{2}+y^{2}=a^{2},
$$

it is easily shown that the coordinates of the first reflection point are

$$
\begin{aligned}
& x_{1}=\partial \cos d_{1} \\
& y_{1}=\partial \sin d_{1} \\
& z_{1}=q_{z 0} \eta 0
\end{aligned}
$$

where

$$
\begin{aligned}
& d_{1}=\tan ^{-1} \frac{y_{0}+q_{y_{0}} \eta_{0}}{x_{0}+q_{x_{0}} \eta 0} \\
& \eta 0=\left(-\beta 0+\sqrt{\left.\beta_{0}^{2}+\alpha \lambda\right) / \alpha}\right. \\
& \beta 0=x_{0} q_{x o}+y_{0} q_{y_{0}} \\
& \alpha=q_{x o}^{2}+q_{y_{0}}^{2}=1-q_{z o}^{2} \\
& \lambda=a^{2}-\left(x_{0}^{2}+y_{0}^{2}\right)
\end{aligned}
$$

The angle $\theta$, which is the angle between the incident ray and the normal to the surface at $x_{1}, y_{1}, z_{1}$ is given by

$$
\cos \theta=\frac{1}{\partial}\left[\beta_{0}+\alpha \eta_{0}\right]
$$


ง

TABLE B.I. COUPLING EFFICIENCIES AS PREDICTED BY OPDEAN AND AN EXACT SOLUTION

\begin{tabular}{|c|c|c|c|}
\hline Image Plane & $\begin{array}{c}\text { Number of } \\
\text { Rays Coupled }\end{array}$ & $\begin{array}{c}\text { Predicted } \\
\text { Efficiency (\%) }\end{array}$ & $\begin{array}{c}\text { Exact } \\
\text { Efficiency }(\%)\end{array}$ \\
\hline 1 & 88 & 58.7 & 59.9 \\
\hline 2 & 70 & 46.7 & 47.4 \\
\hline 3 & 56 & 37.3 & 38.4 \\
\hline 4 & 47 & 31.3 & 31.8 \\
\hline 5 & 39 & 26.0 & 26.7 \\
\hline 6 & 33 & 22.0 & 22.8 \\
\hline 7 & 29 & 19.3 & 19.7 \\
\hline
\end{tabular}




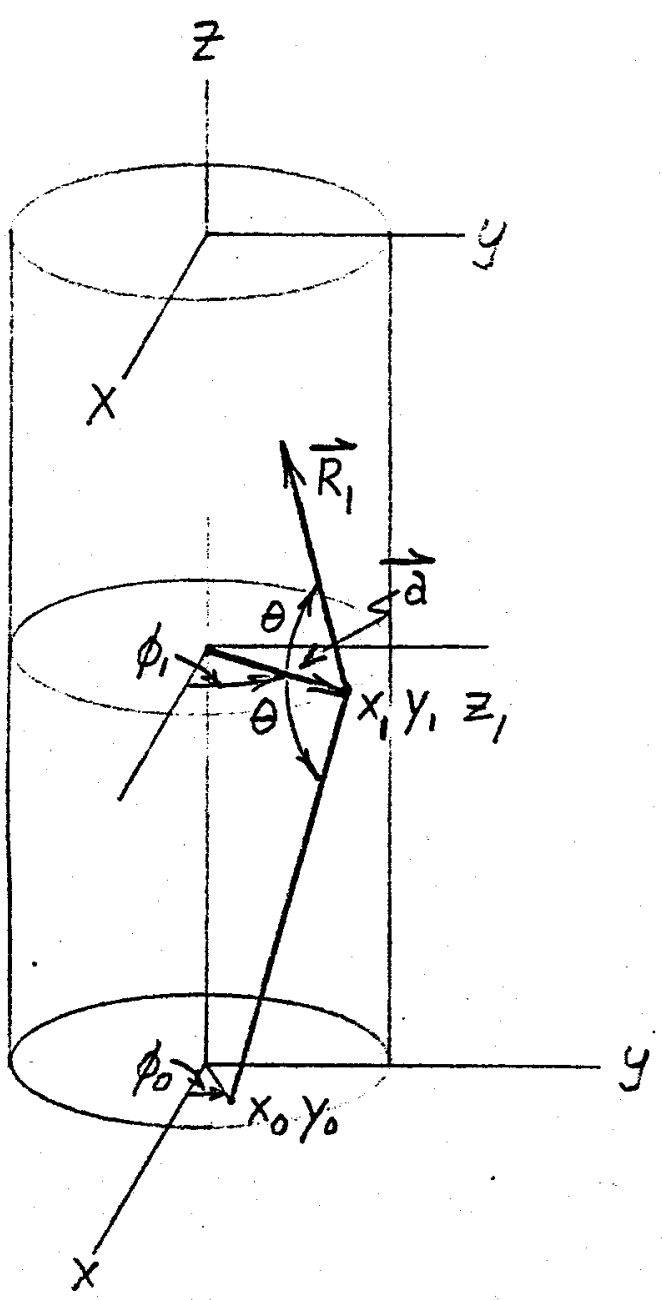

FIGURE B2. SCHEMATIC OF EXACT SOLUTION TO CASE OF RAYS EXITING A CYLINDER FROM POINTS WITHIN THE CYLINDER 
The direction cosines of the reflected ray, $R^{1}$, are determined by the conditions that $R_{1}$ lie in the plane of the radius vector $a$ and the initial ray $R_{0}$, and the reflection angle equal 0 .

Analysis of subsequent reflections shows that at the $n$ 'th reflection point the coordinates are

$$
\begin{aligned}
& x_{n}=\partial \cos d n \\
& y_{n}=\partial \sin d n \\
& z_{n}=z_{1}+(m-1) \Delta z
\end{aligned}
$$

where

$$
\begin{aligned}
& \Delta \mathrm{z}=\mathrm{q}_{\mathrm{zo}} \frac{\alpha \mathrm{a} \cos \theta}{\alpha} \\
& \mathrm{dn}=\mathrm{d}_{1}+(\mathrm{n}-1) \Delta \mathrm{d} \\
& \Delta \mathrm{d}=\tan ^{-1}\left[\frac{\mathrm{y}_{1}+\mathrm{q}_{\mathrm{yo}} \eta_{1}-\mathrm{y}_{1}\left(\alpha \eta_{1}^{2} / \mathrm{a}^{2}\right)}{\mathrm{x}_{1}+\mathrm{q}_{\mathrm{xo}} \eta_{1}-\mathrm{x}_{1}\left(\alpha \eta_{1}^{2} / \mathrm{a}^{2}\right)}\right]-\mathrm{d}_{1} \\
& \eta_{1}=2\left(\eta_{0}+\beta 0 / \alpha\right)
\end{aligned}
$$

The direction cosines of the ray leaving the $n$ 'th reflection point are

$$
\begin{aligned}
& q_{x n}=\frac{\alpha}{2 \cos \theta}[\cos (d n+\Delta d)-\cos d n] \\
& q_{y n}=\frac{\alpha}{2 \cos \theta}[\sin (d n+\Delta d)-\sin d n] \\
& q_{z n}=q_{z 0}
\end{aligned}
$$

The number of reflections in a cylinder of length is the largest integer $\mathbf{n}$ such that

$$
n \leqslant 1+\left(\ell-z_{1}\right) / \Delta z
$$

If $x_{n}, y_{n}, z_{n}$ are the coordinates of the last reflection point then the coordinates of the ray in the exit plane are

$$
\begin{aligned}
& x=x_{n}+\frac{q_{x n}}{q_{z n}}\left(l-z_{n}\right) \\
& y=y_{n}+\frac{q_{y n}}{q_{z n}}\left(l-z_{n}\right) \\
& z=l
\end{aligned}
$$

and $q_{x n}, q_{y n}, q_{z n}$ are the final direction cosines of the ray.

To apply this solution in a ray trace program we have prepared the subroutine listed in Figure B3. This routine accepts as input data the fiber radius $A$, length $X L$, the initial coordinates $X I$ and $Y I$, and the initial values of the direction cosines, supplied as a three element array, QTI. Output data are the number of reflections NR, the final values of $X$ and $Y$, and the final values of the direction cosines, $Q T$. Input/Output lists for several sample problems are presented in Figure B4. 
0001

0002

0003

0004

0005

0006

0007

0008

0009

0010

0011

0012

0013

0014

0015

0016

0017

0018

0019

0020

0021

0022

0023

0024

0025

0026

0027

0028

0029

0030

0031

0032

0033

0034

0035

0035

0037

0038

0039

0040

0041

0042

0043

0044

0045

0046

0047

0048

0049

SUEROUITINE FIEEF ( $A, X L, X I, Y I, Q T I, X, Y, Q T, N R$ )

C $\quad 14$ JแLY 77

C

DIMENSION QTI (3), QT (3)

QXO=QTI $(1)$

QYO=QTI (2)

$Q Z O=Q T I(3)$

$A L P=1.0-9 ! 2 O * Q Z O+1.0 E-3$

$E E T=X I * D X O+Y I * Q Y O$

$G A M=A * A-X I * X I-Y I * Y I$

$E T A=(-E E T+S Q R T(E E T * E E T+A L P * G A M)) / A L P$

$W=(E: E T+A L F * E T A) / A$

$Z 1=Q Z Z O E E T A$

IF $(Z 1-X L) \quad 10,10,5$

$8 \quad \operatorname{QT}(1)=\mathrm{QTT}(1)$

$\operatorname{QT}(z)=\mathrm{QTT} I(2)$

QT $(3)=Q T I(3)$

$X=X I+Q T(1) * X L / Q T(3)$

$Y=Y I+Q T(2) * X L / Q T(3)$

NR $=0$

GD TO 20

$10 \quad X 1=X I+D X O * E T A$

$Y 1=Y I+Q Y O * E T A$

$E T A=2 . O *(E T A+E E T / A L P)$

$X Z=X 1+0 \times 0 * E T A-A L F * X 1 * E T A * E T A /(A * A)$

$Y Z=Y 1+Q Y O * E T A-A L F * Y 1 * E T A * E T A /(A * A)$

$A N G 1=A T A N 2(Y 1, X 1)$

ANGZ=ATANZ $(Y Z, X Z)$

DELL $=A N G Z-A N G 1$

$\square Z=2.0 * A * W * Q Z O / A L P$

$F N R=1 . O+(X L-Z 1) / D Z$

$N R=I F I X(F N R)$

FNR $=F L O A T$ (NR)

ANGZ=ANG $1+(F N F-1.0) *$ LEL

$Z N=Z 1+(F N R-1.0) * D Z$

$X N=A * C D S(A N G Z)$

$Y N=A * S I N(A N G 2)$

$Q X=A L F *(\operatorname{COS}(A N G 2+\square E L)-\operatorname{COS}(A N G 2)) /(2.0 * W)$

$Q Y=A L F *(S I N(A N G Z+[E L)-S I N(A N G 2)) /(2 . O * W)$

$Q Z=Q Z O$

$\operatorname{QT}(1)=\mathrm{QX}$

$\mathrm{QT}(2)=\mathrm{QY}$

$\mathrm{QT}(3)=Q Z$

$X=X N+Q X *(X L-Z N) / Q Z$

$Y=Y N+Q Y *(X L-Z N) / Q Z$

20 CONTINUE

RETURN

END

END\$

$*$ END-OF-TAPE

*END

FIGURE B3. IISTING OF SUBROUTINE FIBER 
$A=50.0 \quad X L=.50 E+05 \quad X I=\quad .0000 \quad Y I=\quad .0000$

$\operatorname{QTI}(1)=.000000$ QTI(2) $=.000000$ QTI $(3)=1.000000 \quad N R=0$

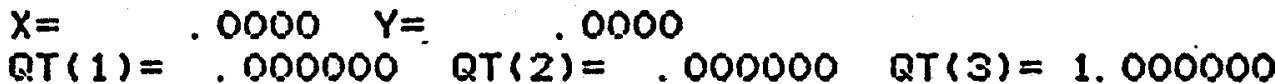

$A=50.0 \quad X L=. .50 E+05 \quad X I=\quad .0000 \quad Y I=\quad .0000$

$\mathrm{QTI}(1)=.000000$ QTI $(2)=.173647$ QTI $(3)=.984808 \quad \mathrm{NR}=88$

$X=\quad .0000 \quad Y=16.2838$

$\operatorname{QT}(1)=.000000 \quad \mathrm{RT}(2)=.173647$ QT $(3)=.984508$

$A=50.0 \quad X L=.50 E+05 \quad X I=\quad .0000 \quad Y I=\quad .0000$

$\operatorname{QTI}(1)=.000000$ QTI $(2)=.087153$ QTI(3)=.996195 NR= 44

$X=.0000 \quad Y=-25.6973$

$\operatorname{QT}(1)=.000000 \quad$ QT $(Z)=.087153 \quad$ QT $(3)=.996195$

$A=50.0 \quad X L=.50 E+05 \quad X I=17.6770 \quad Y I=17.6770$

QTI $(1)=.000000$ QTI $(2)=.000000$ QTI $(3)=1.000000 \quad N R=0$ $X=17.6770 \quad Y=17.6770$

$\operatorname{QT}(1)=.000000 \quad \operatorname{QT}(2)=.000000 \quad \operatorname{QT}(3)=1.000000$

$A=50.0 \quad X L=.50 E+05 \quad X I=17.6770 \quad Y I=17.6770$

$\operatorname{RTI}(1)=.000000$ QTI $(Z)=.173647$ QTI $(3)=.984908$ NR= 34 $X=-31.2214 \quad Y=\quad 31.9484$

QT $(1)=-.160607 \quad \operatorname{QT}(2)=.066017$ QT $(3)=.984808$

$A=50.0 \quad X L=.50 E+05 \quad X I=.17 .6770 \quad Y I=17.6770$

DTI $(1)=.000000$ QTI $(2)=.097153$ QTI $(3)=.996195 \quad N R=47$ $X=17.1834 \quad Y=6.1128$

QT $(1)=-.049529 \quad$ QT $(2)=.072392 \quad$ QT $(3)=.996195$

$A=.50 .0 \quad X L=.50 E+05 \quad X I=31.8198 \quad Y I=31.8198$

$\operatorname{QTI}(1)=.000000$ QTI $(2)=.000000$ QTI $(3)=1.000000 \quad N R=0$ $X=31.8198 \quad Y=31.8198$

$\operatorname{QT}(1)=.000000 \quad$ QT $(2)=.000000 \quad$ QT $(3)=1.000000$

$A=50.0 \quad X L=.50 E+05 \quad X I=31.8198 \quad Y I=\quad 31.8198$

$\operatorname{QTI}(1)=.000000$ QTI $(2)=.173647$ QTI $(3)=.984808 \quad \mathrm{NR}=115$

$X=22.7971 \quad Y=31.6500$

QT $(1)=-.173643$ QT $(2)=.001299$ QT $(3)=.984308$

$A=50.0 \quad X L=.50 E+05 \quad X I=31.8196 \quad Y I=31.8198$

QTI $(1)=.000000$ QTI $(2)=.087153$ QTI $(3)=.996195 \mathrm{NR}=57$

$X=32.5937 \quad Y=\quad 6.1613$

QT $(1)=.008661$ QT $(2)=.086722$ QT $(3)=.996195$

$A=50.0 \quad X L=.50 E+05 \quad X I=31.8198 \quad Y I=\quad 31.3198$

QTI $(1)=.173647^{\circ}$ QTI $(Z)=.000000$ QTI $(3)=.984808 \quad N R=115$

$X=31.6504 \quad Y=22.7980$

QT $(1)=.001303 \quad$ QT $(2)=-.173641 \quad$ QT $(3)=.984303$

$A=50.0 \quad X L=.50 E+05 \quad X I=31.8198 \quad Y I=31.8198$

RTI $(1)=.0 \$ 7153 \cdot$ QTI $(2)=.000000$ QTI $(3)=.996195 \quad N R=57$

$X=$ 6. $1613 \quad Y=32.5937$

$\operatorname{QT}(1)=.086722 \quad \operatorname{QT}(2)=.009661 \quad$ QT $(3)=.996195$

FIGURE B4. INPUT/OUTPUT DATA FOR SAMPLE PROBLEMS USING SUBROUTINE FIBER 


\section{APPENDIX C \\ INVESTIGATION OF THE HIGH TEMPERATURE PERFORMANCE OF GaAs UGHT EMITTING DIODES}

Because of their low power requirements and small size, solid-state luminescent devices that emit in the near infrared offer an attractive possibility for the conversion of electrical signals to optical signals for transmission over large distances through optical fibers. Whether this concept will prove useful in geothermal well-logging depends, in part, on the performance of such devices at high temperatures. In this Appendix some of the fundamental physical processes that limit the high temperature efficiency of both solid-state lasers and light emitting diodes (LEDs) are discussed. It should be noted, however, that in addition to the fundamental limitations discussed here, there are other problems of an engineering nature that must also be considered in the design of an optical source for down-hole operation. Thus, the possibilities raised here should be taken as indications of what might be achieved if the mechanical and electrical problems associated with operation in a high temperature, corrosive environment can be solved.

\section{A. Theory}

A simple model that illustrates the main features of solid-state luminescence is shown in Figure C1. Here $\mathrm{C}$ represents the generation of electron-hole pairs by some excitation process, usually minority carrier injection in junction devices. The decay path $R$ represents luminescent recombination of an electron initially in the conduction band with a hole at level $L$, while the non-radiative path NR represents recombination via other mechanisms such that no luminescence occurs. If we let $P_{r}$ be the probability of luminescent decay, then let $P_{n r}$ be the non-radiative recombintion probability, then the luminescent efficiency is

$$
\eta=\frac{\mathrm{P}_{\mathrm{r}}}{\mathrm{P}_{\mathrm{r}}+\mathrm{P}_{\mathrm{nr}}}
$$

This quantity is the fraction of electron-hole pairs that recombine via photon emission; it is, therefore, also a measure of the fraction of input (electrical) energy that is converted to useful radiant energy.

Consideration of the kinetic equations governing electron-hole recombination leads to the following expression for the luminescent efficiency: (C1)

$$
\eta=\left[1+\left(\frac{m_{h}}{m_{e}}\right)^{3 / 2} \frac{N_{T} \sigma_{p} T}{N_{L} \sigma_{n} L} e^{-\beta\left(E_{R}-E_{t}\right)}\right]^{-1}
$$

Here $m_{e}$ and $m_{h}$ are the effective masses of electrons and holes, respectively, $N_{T}$ and $N_{L}$ are the concentrations of levels of types $T$ and $L, \sigma_{p}^{T}$ is the hole capture cross section for $T$ centers while $\sigma_{m}$ is the corresponding electron cross section for $L$ levels, $\beta$ is $1 / k T$, where $k$ is the Boltzmann constant and $T$ is the absolute temperature, and the energies $E_{1}$ and $E_{t}$ are defined in Figure $C 1$. It is evident from this result that high efficiency requires $N_{T} \varangle 4 N_{L}$ and $E_{1}-E_{t} \rightarrow k T$. Thus, for good luminescent efficiency one re quires (1) a high concentration of luminescent centers as compared with the concentration of traps leading to non-radiative recombination, and (2) shallow non-radiative traps and deep radiative acceptor levels. A deep luminescent level is also desirable for another reason, namely, that in such a case the energy of the emitted photon is significantly less than the band gap energy, and the probability that the photon will be absorbed is therefore small. (C2) 


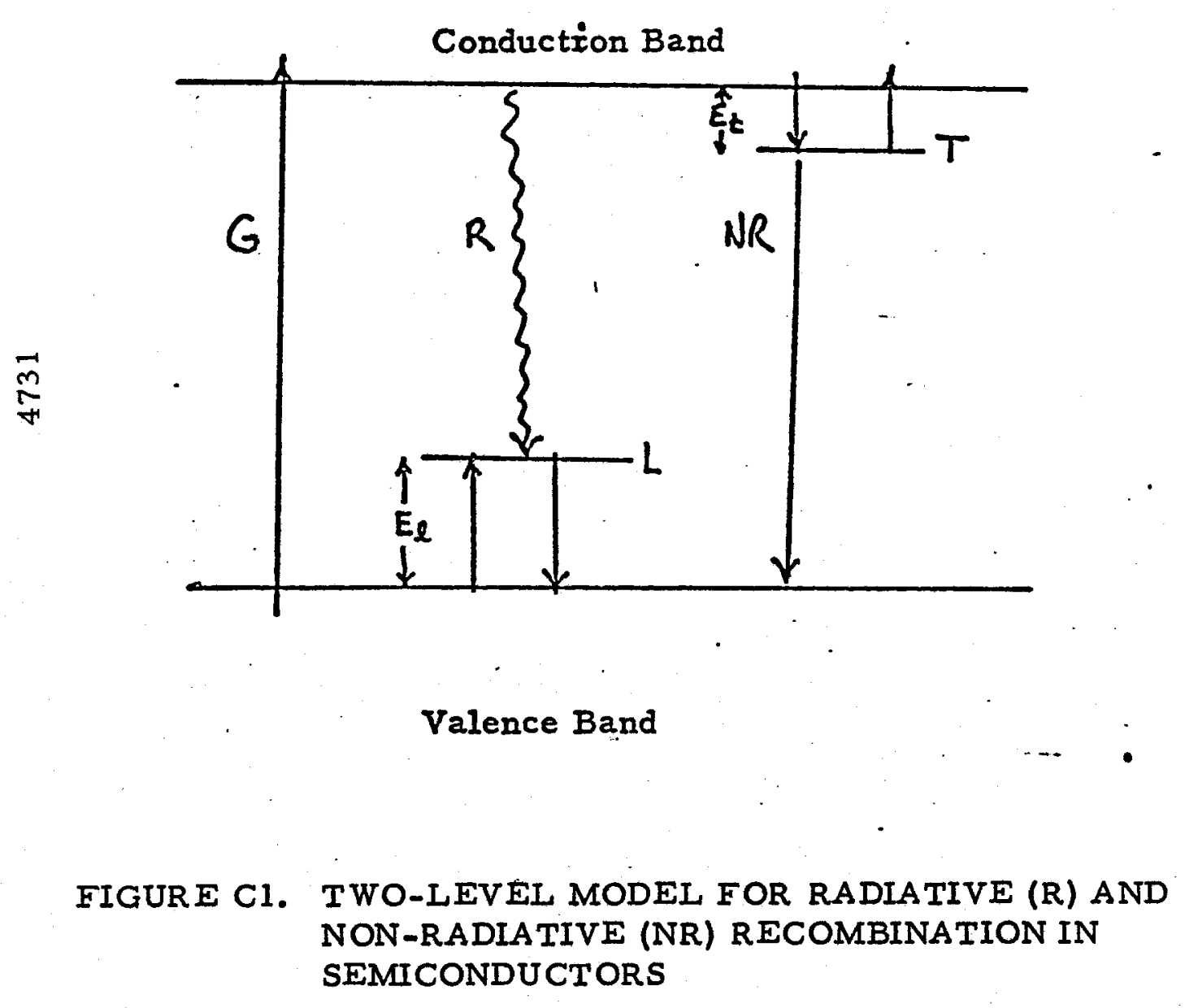


If the radiative and non-radiative levels are deep and shallow, respectively, then $E_{1}-E^{t}-0$ and the exponential term increases as the temperature increases, thus leading to a decrease in efficiency. Physically, this drop in efficiency is caused to some extent by a decrease in the hole population of luminescent centers and, to a greater extent, by an increase in the electron population in non-radiative traps. These population changes tend to make the radiative path less probable and the non-radiative path more probable as the temperature increases.

There is little that one can do about this temperature quenching effect except to minimize the concentration of non-radiative centers (which is an extremely difficult technological problem) and, perhaps, increase the concentration and level depth of luminescent centers. Unfortunately, increasing the depth of

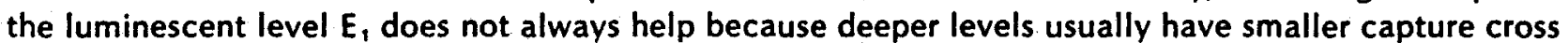
sections and this tends to decrease, rather than increase the efficiency. Heavier concentrations of luminescent centers also lead to complications through the formation of impurity bands (rather than isolated energy levels) that merge with the conduction and valence bands at very high concentrations. (C3) Thus, while the simple two-trap model may suffice for illustrative purposes, it is not always reliable as a guide to the design of more efficient devices.

There is, on the other hand, some experimental evidence that the introduction of deep luminescent centers is advantageous to high temperature performance. For example, when GaAs is heavily doped with $\mathrm{Si}$, a quasi-continuous distribution of acceptor and donor levels is produced, with some acceptor levels lying deep within the energy gap. ${ }^{(C 3)} A$ rather complicated analysis ${ }^{(C 3)}$ shows that presence of these deeper acceptor states can account for the fact that GaAs:Si diodes are the most efficient devices yet realized. (C4) Supporting evidence that the deeper levels are involved is found in the fact that the emission spectrum shifts to longer wavelengths as the Si concentration is increased. (C5) Also, experimental studies of the temperature dependence ${ }^{(\mathrm{C5})}$ and time dependence ${ }^{(\mathrm{C} 6)}$ (in the case of pulsed operation) of the luminescent spectrum indicate that the deeper levels are less susceptible to thermal quenching than are shallow luminescent levels. Unfortunately, we have yet to find any direct experimental evidence that heavy Si doping is beneficial to operation at temperatures of interest here. This, therefore, is one investigation that should definitely be pursued.

It is also possible that some other semiconductor with deep luminescent levels could prove superior to GaAs at high temperatures. In particular, luminescence in $\mathrm{Cu}$ doped $\mathrm{CdS}$ is known to persist at temperatures as high as $300^{\circ} \mathrm{C}$ with little loss of efficiency. $(\mathrm{C7})$ The main difficulty with $\mathrm{CdS}$, and most other II-VI semiconductors as well, is that it is difficult to fabricate efficient junction devices from such

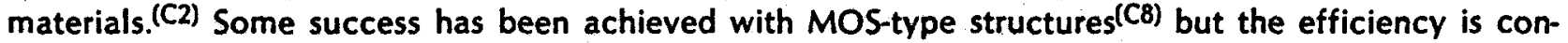
siderably lower than it is for CaAs diodes at room temperature. Still, the possibility that a CdS device may show less temperature degradation suggests that such devices are worthy of further study.

\section{B. Experimental Investigation}

To further explore the possibility that the high temperature performance of a CaAs diode may be adequate, an experimental investigation of a commercial device was conducted.

A Texas Instrument TIXLI6A PN GaAs LED was selected for the experiment because of its exceptionally effective heat sink. The LED was attached to an additional external aluminum heat sink along with a thermocouple probe. Both the LED and thermocouple were attached with Wakefield Thermal Compound \#120-2 to ensure low thermal resistance between the components. Figure C2 shows the LED mounted to its aluminum holder, and the LED assembly with thermocouple mounted in position in the temperature chamber.

Light from the LED in the oven was coupled to the monochromator and detector via a 1.5M length of CCW graded index optical fiber. Rigid mechanical supports were used on both ends of the fiber, providing stable coupling conditions throughout the course of the experiment. 


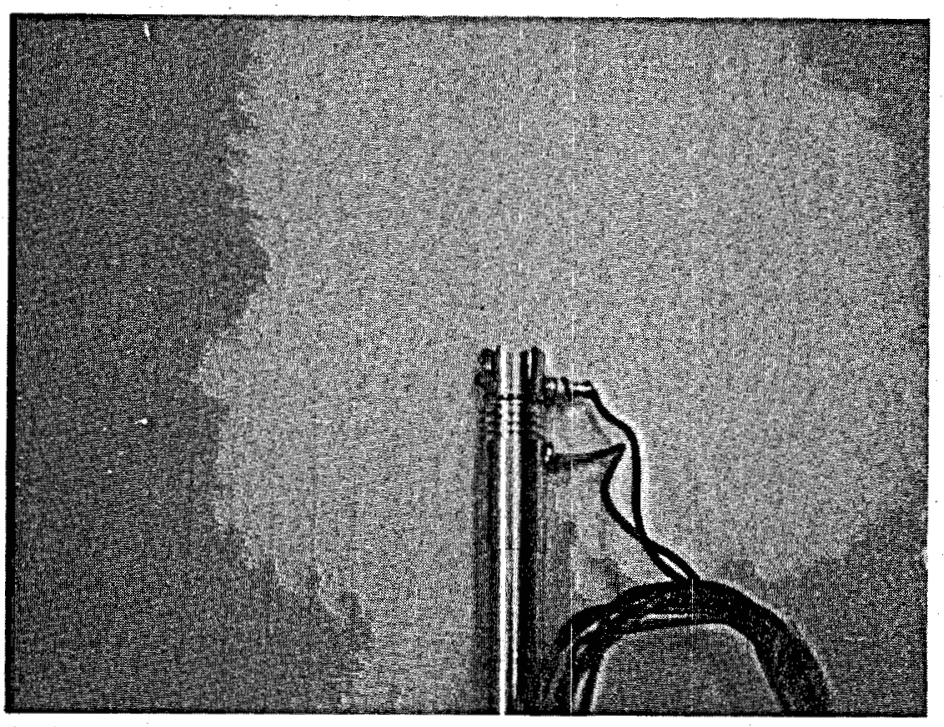

(a)

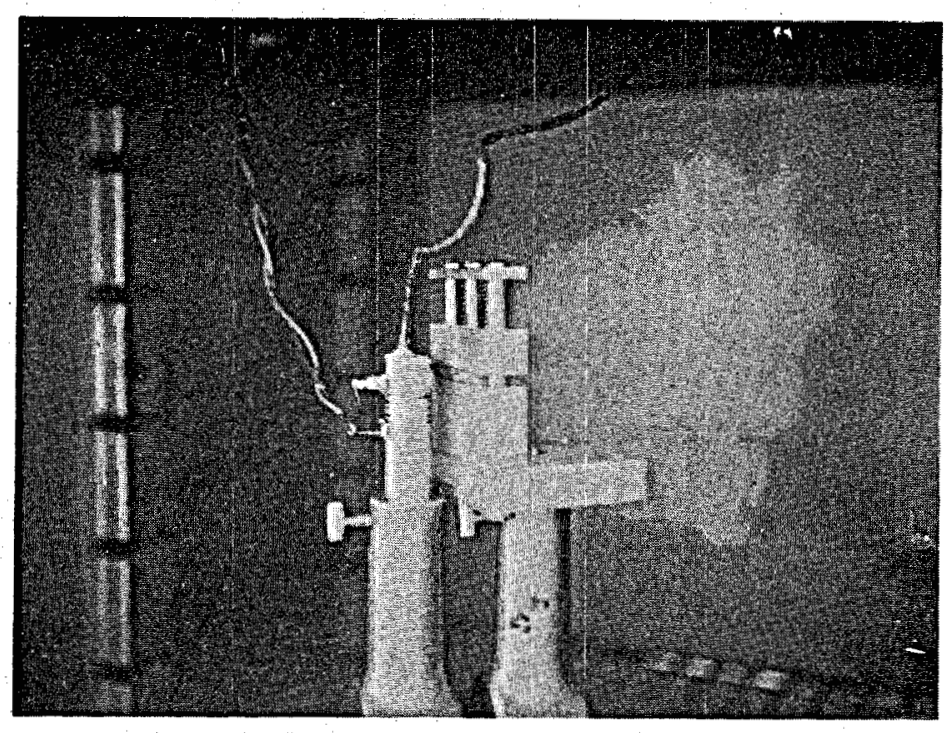

(b)

FIGURE C2. EXPERIMENTAL ARRANGEMENT FOR DETERMINING T EMPERATURE EFFECT ON EMISSION OF GaAs LED;

a) LED and thermocouple mounted on Al heat sink, b) optic fiber clamped to accept light output 
The receiving optics consisted of the fiber mechanical support, a $10 \times$ microscope objective used to collect and collimate the output of the fiber, a Bausch \& Lomb monochromator, and a UDT500 silicon photodiode and preamplifier. A 20-turn, $0.1 \%$ linear potentiometer was mechanically linked to the wavelength select knob on the monochromator to provide an analog signal proportional to wavelength. The output of the UDT500 was connected to the $Y$ input of an $X-Y$ recorder, and the wiper of the potentiometer was connected to the $X$ input. Voltage across the pot and the sensitivity and centering controls of the recorder were adjusted so that all the data could be displayed conveniently on an 8-1/2 $\times 11$ sheet of graph paper by the recorder.

Spectral data were taken with the monochromator from $30^{\circ} \mathrm{C}$ to $210^{\circ} \mathrm{C}$ in increments of $30^{\circ} \mathrm{C}$. This data is presented in Figure C3. Note that the effects of increasing temperature are twofold: (1) a spectral shift in the diode output further towards the infrared, and (2) a decrease in total integrated light output.

The data in Figure $\mathrm{C} 3$ is the actual output voltage from the silicon detector and preamplifier assembly. In this region of the spectrum, the response of the silicon detector is declining, which accounts for part of the apparent output decline with temperature. To compensate for this effect, the data were normalized to the photodiode response curve, yielding the corrected plot shown in Figure C4. Note that the peak output at $210^{\circ} \mathrm{C}$ is down only approximately a factor of 5 from the peak output at $30^{\circ} \mathrm{C}$. This represents about $14 \mathrm{~dB}$. loss in optical signal, which is tolerable in most optical communication systems.

To further analyze this data, the curves in Figure $\mathrm{C} 4$ were integrated to obtain the total integrated output from the LED at each of the test temperatures. These values are plotted as a function of absolute temperature in Figure $\mathrm{C} 5$. For illustrative purposes, values for a GaAs:Zn diode reported by Carr (C10) are included in the plot. The values obtained in this experiment agree closely with those obtained by Carr, where they overlap in the region from $300^{\circ} \mathrm{K}$ to $360^{\circ} \mathrm{K}$

Finally, in Figure C6, we show the peak position of the spectral output plotted as a function of absolute temperature. There are two points to be noted here, the first being that the luminescent output falls off as $\exp (-T / \theta)$, which does not agree with the theoretical model described earlier. This discrepancy has been noted before and is apparently caused by increased absorption in the diode itself as the energy gap becomes smaller with increasing temperature.(C11) This means that the output of any such light emitting device will fall off more rapidly with temperature than would be the case if there were no selfabsorption of the luminescent emission. However, because this effect occurs for both deep and shallow centers, it has no bearing on the relative merits of deep vs. shallow states at high temperature.

The second point to be noted is that at room temperature the diode with unknown dopant emits at a slightly longer peak wavelength than the $\mathrm{Zn}$ doped diode. This indicates that the acceptor level created by the unknown dopant is slightly deeper than the $\mathrm{Zn}$ level. Comparison with experimental data published by Ladany(C12), who studied the emission spectrum of GaAs:Si as a function of Si concentration, suggests that the unknown dopant may be $\mathrm{Si}$, in a concentration between $0.25 \%$ and $0.5 \%$ by weight $\mathrm{Si}$ in $\mathrm{Ga}$ in the melt. This tends to agree with Moruzumi and Takahashi(C5) who found that with low Si concentration the peak wavelength varies with temperature in much the same way as is illustrated here.

The interesting point, however, is that if the unknown dopant is indeed $\mathrm{Si}$, it must be present in a rather low concentration. According to the published data cited above ${ }^{(\mathrm{C} 9, \mathrm{C12})}$ diodes with much higher $\mathrm{Si}$ concentrations can be fabricated, and would result in (1) a shift in the peak emission to longer wavelengths, and (2) a more complex dependence of the output of temperature. However, the most encouraging aspect of this conjecture is that the data of Moruzumi and Takahashi( ${ }^{(C 5)}$, which was obtained in the $77^{\circ} \mathrm{K}$ to $300^{\circ} \mathrm{K}$ range, indicate that the emission from heavily doped diodes changes very little with temperature. If, as theory suggests, this trend persists at higher temperatures, then the high temperature performance of heavily doped GaAs:Si diodes should be significantly better than is indicated in Figure C6. 


$$
\text { C * }
$$

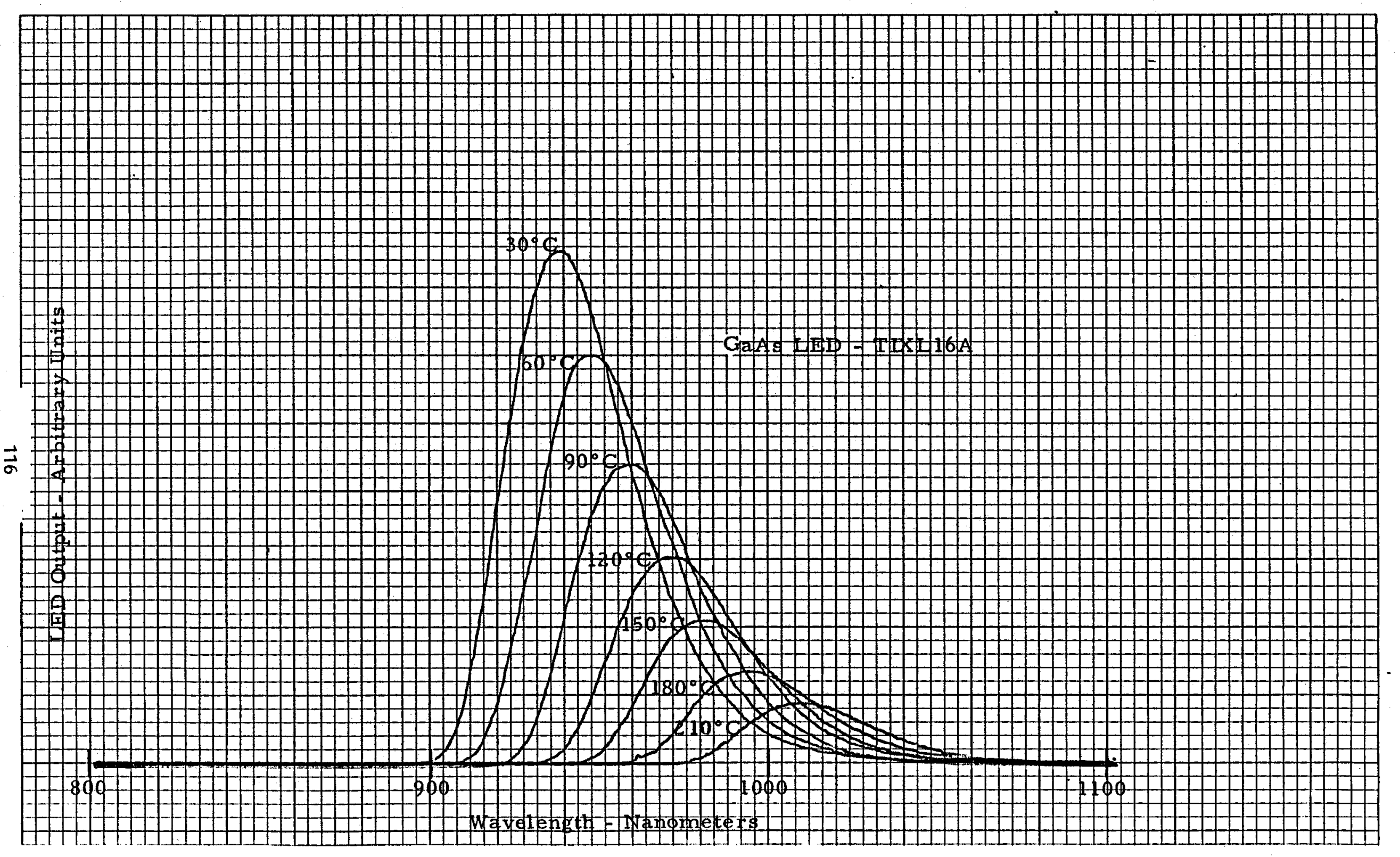

FIGURE C3. OUTPUT OF GaAs PN LED AS A FUNCTION OF WAVELENGTH AND TEMPERATURE 


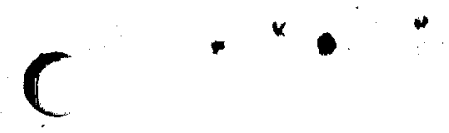

4734

\section{GAAB LED}

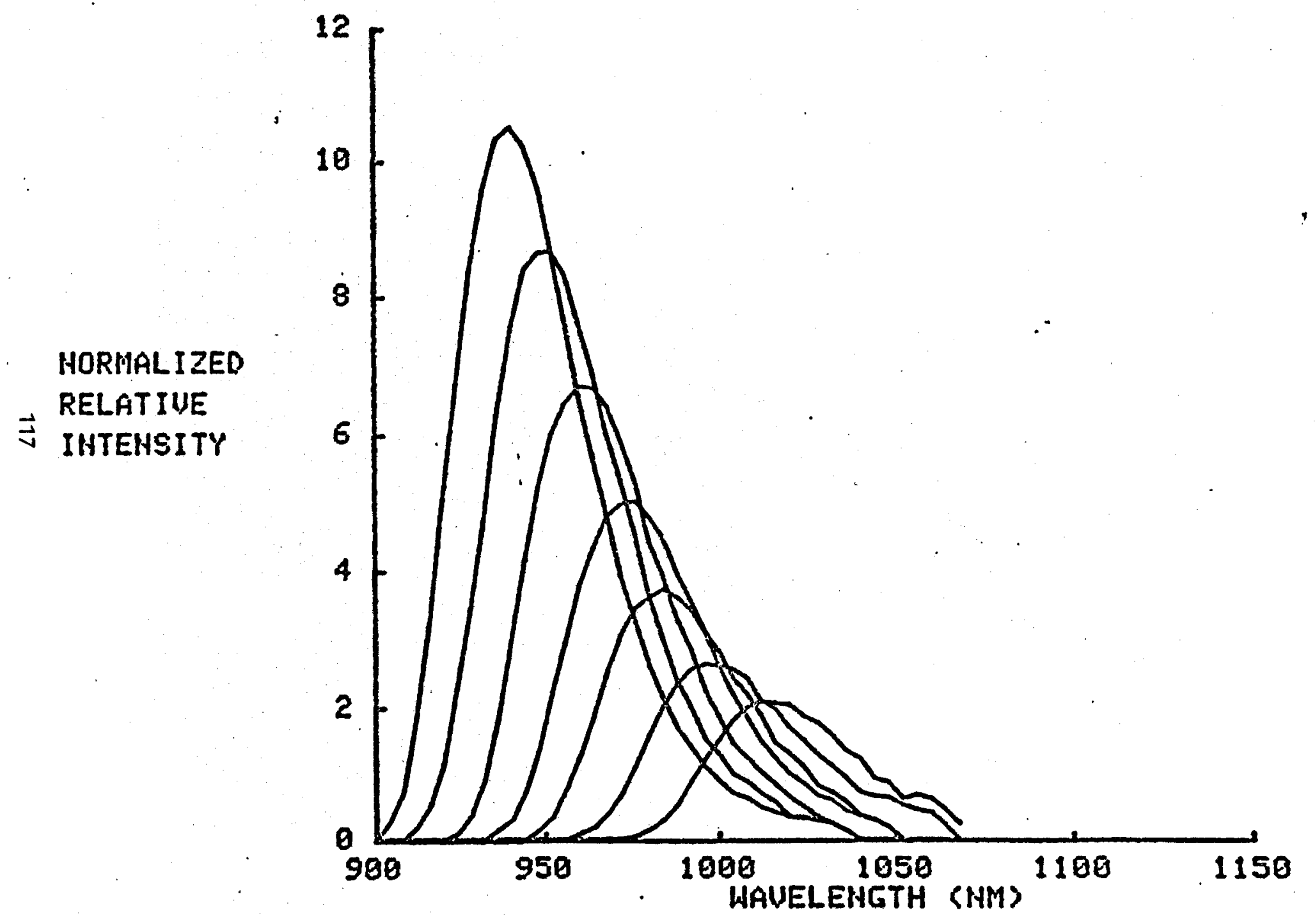

FIGURE C4. NORMALIZED OUTPUT OF GaAs PN LED AS A FUNCTION OF WA VELENGTH AND TEMPERATURE 
- * .

A)

$0 \quad \therefore \quad x$

4736

FIGURE C6: POSITION OF PEAK OUTPUT OF GAAS LED AS A FUHCTIOH OF TEMPERATURE WITH LEAST SQUARES FIT TO DATA.

ADDITIOHAL DATA DUE TO CARR AHD PITTMAHCGAGS:ZH).

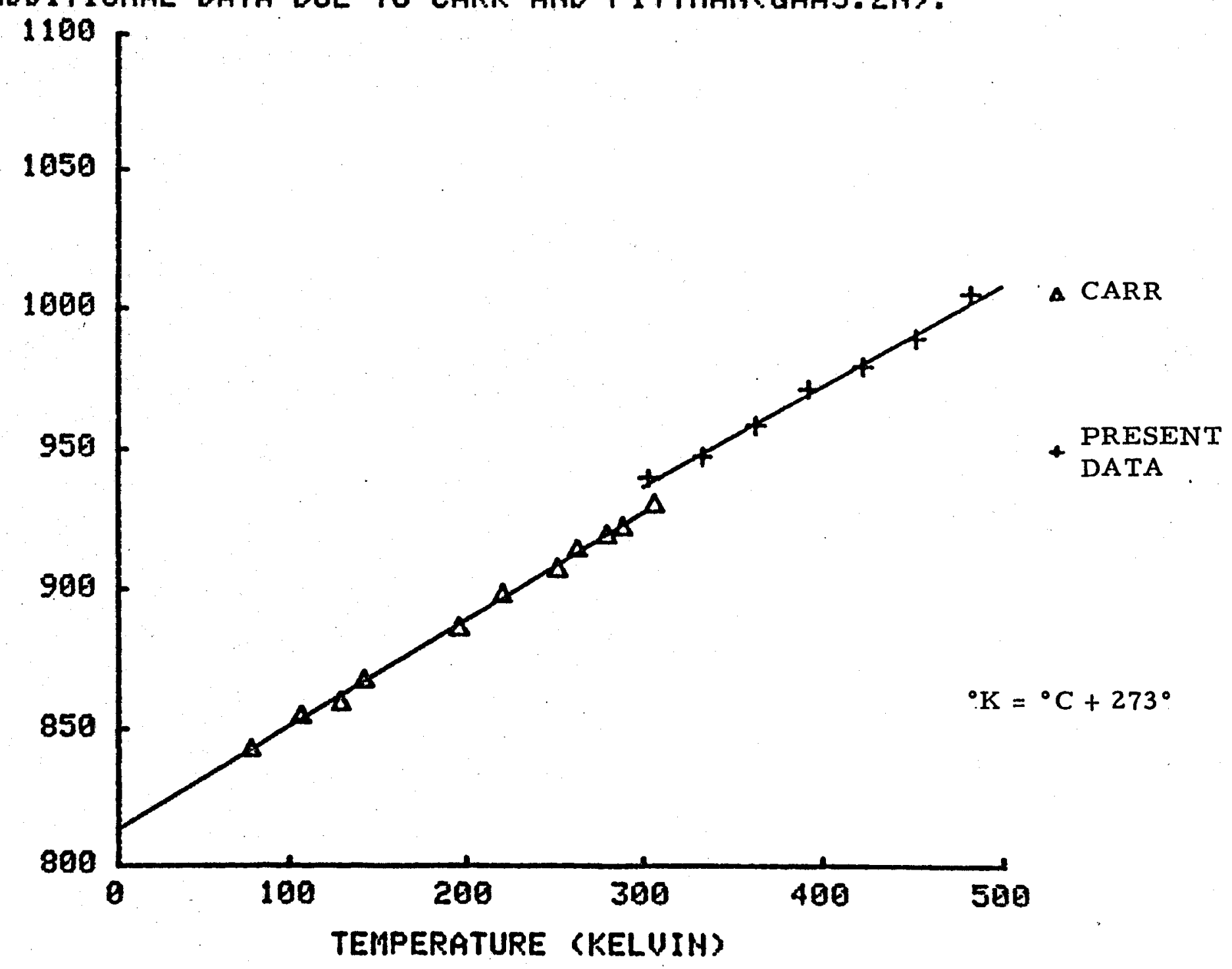

$\overrightarrow{\vec{\omega}}$

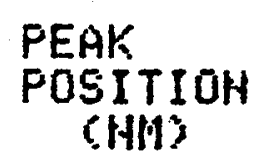




\section{REFERENCES FOR APPENDIX C}

C1. H. F. Ivey, in "Solid-State Physics in Electronics and Telecommunications," M. Desirant and J. L. Michiels, eds., Vol. 4, p. 611, Academic Press, New York, 1960.

C2. P. J. Dean, in "Applied Solid-State Science," R. Wolfe, ed., Vol. 1, p. 1, Academic Press, New York, 1969.

C3. F. Stern, Phys. Rev. 148, 186 (1966).

C4. H. F. Matare, Adv. Electronics and Electron Phys. 42, 179 (1976).

C5. T. Moruzumi and K. Takahashi, Japan. J. Appl. Phys. 8, 348 (1969).

C6. D. Redfield, J. P. Wittke and J. I. Pankove, Phys. Rev. B 2, 1830 (1970).

C7. D. Curie, "Luminescence in Crystals," John Wiley and Sons, New York (1963).

C8. J. H. Yee and C. A. Condas, Solid State Elec. 11, 419 (1968).

C9. S. A. Cerwin, Memorandum dated 4 May 1977.

C10. W. N. Carr, IEEE Trans. Electron Devices, ED-12, 531 (1965).

C11. J. I. Pankove, "Optical Processes in Semiconductors," Dover, New York (1971).

C12. I. Ladany, J. Appl. Phys. 42, 654 (1971). 